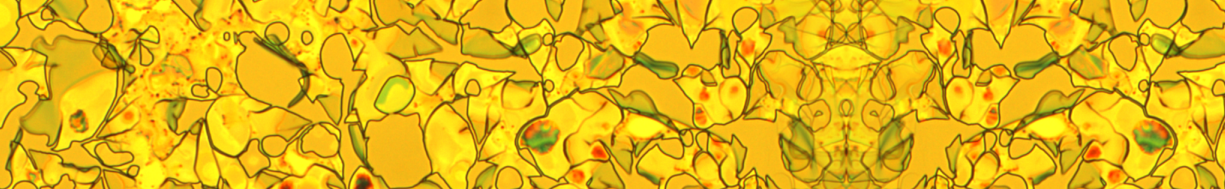

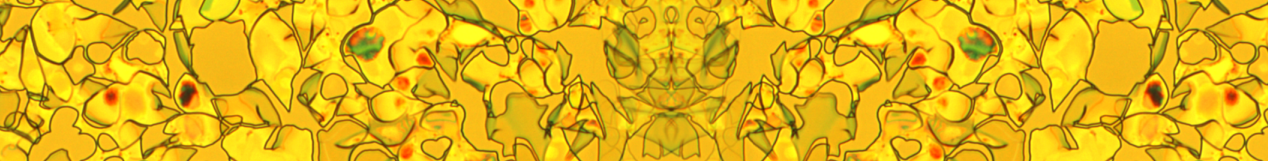
S.

(a) $(0)$ iv

\title{
Microfabrication
}

\section{for solar-to-hydrogen devices}

in 0 .

$$
\text { (1) }
$$

\section{P.J. Westerik}

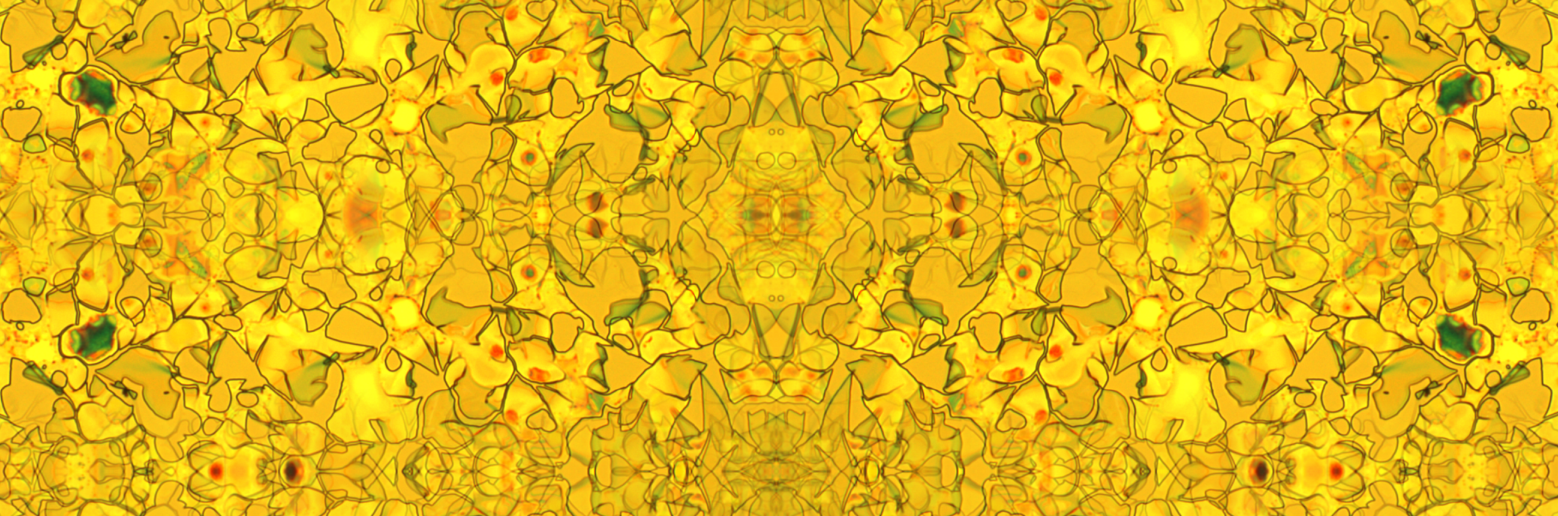

a. 
Microfabrication for solar-to-hydrogen devices

\author{
P.J. Westerik
}




\section{Promotiecommissie:}

Prof. dr. ir. J.W.M. Hilgenkamp (voorzitter) Universiteit Twente

Prof. dr. J.G.E. Gardeniers (promotor) Universiteit Twente

Prof. dr. ir. J. Huskens (promotor) Universiteit Twente

Prof. dr. R. van de Krol Helmholz Zentrum Berlin

Dr. A. Bieberle (referent) DIFFER Eindhoven Dr. M. Creatore Technische Universiteit Eindhoven

Prof. dr. J. Schmitz Universiteit Twente

Prof. dr. ir. M. Huijben Universiteit Twente

Prof. dr. G. Mul Universiteit Twente

This work is part of the research program of the Netherlands Organization for Scientific Research (formerly FOM, now part of NWO, projects 13CO12-1 and 13CO12-2).

Microfabrication for solar-to-hydrogen devices

ISBN: 978-90-365-4552-5

DOI: $10.3990 / 1.9789036545525$

Cover art: Pieter Westerik

Printed by: Gildeprint - www. gildeprint.nl

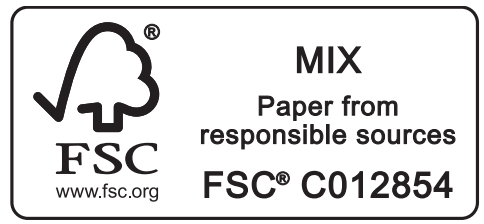

(C)Copyright 2018 Pieter Westerik 


\section{MICROFABRICATION FOR SOLAR-TO-HYDROGEN DEVICES}

\section{PROEFSCHRIFT}

ter verkrijging van

de graad van doctor aan de Universiteit Twente, op gezag van de rector magnificus,

prof. dr. T.T.M. Palstra,

volgens besluit van het College voor Promoties

in het openbaar te verdedigen op vrijdag 8 juni 2018 om 14:45 uur

door

Pieter Johannes Westerik geboren op 26 augustus 1988

te Ngqeleni, Zuid-Afrika 
Dit proefschrift is goedgekeurd door de promotoren:

Prof. dr. J.G.E. Gardeniers

Prof. dr. ir. J. Huskens 


\section{Contents}

1 Introduction

1.7 Solar water splitting . . . . . . . . . . . . . . . . . . 11

1.2 Microfabrication . . . . . . . . . . . . . . . 13

1.3 Thesis outline . . . . . . . . . . . . . . . . . . . . . . . 14

2 Performance considerations $\quad 15$

2.1 Light absorption . . . . . . . . . . . . . . . . . . . . 15

2.1 .1 Reflection .................. . 16

2.1 .2 Transmission .................. . . 17

2.1 .3 Parasitic light absorption . . . . . . . . . . . 20

2.2 Charge separation . . . . . . . . . . . . . . . . 21

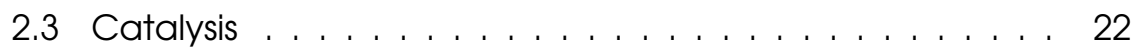

2.4 Ion transport . . . . . . . . . . . . . . . . . . . . . . . . . 24

2.5 Gas crossover . . . . . . . . . . . . . . . . . . . . . 26

2.6 Conclusion . . . . . . . . . . . . . . . . . . . . . 27

3 Microfabrication for vertical structures $\quad 29$

3.1 Comparison to other 3D microfabrication techniques ... 30

3.2 Modify the top part of a structure . . . . . . . . . . . . . 33

3.2 .1 Fabrication . . . . . . . . . . . . . . . . 33

3.2 .2 Fabrication details $\ldots \ldots \ldots$ 
3.2 .3 Results and discussion . . . . . . . . . . . . . . . . 37

3.3 Selective opening of top surfaces . . . . . . . . . . . . . . 39

3.4 Modify the bottom part of a structure . . . . . . . . . . 47

3.4 .1 Fabrication . . . . . . . . . . . . . . . . . . . . . 47

3.4 .2 Results and discussion . . . . . . . . . . . . . . . . . 48

3.5 Modification at an intermediate height . . . . . . . 51

3.5.1 Fabrication . . . . . . . . . . . . . . . . . . 51

3.5 .2 Fabrication details $\ldots \ldots \ldots$. . . . . . . . . . . . . . 52

3.5.3 Results and discussion . . . . . . . . . . . . . . . . 54

3.6 Conclusion . . . . . . . . . . . . . . . . . . . . . . . . 57

4 Microfabrication of repeating structures 59

4.1 Introduction . . . . . . . . . . . . . . . . . . . . 60

4.2 Materials and Methods . . . . . . . . . . . . . . . . . . 62

4.2.1 Patterning of the mask . . . . . . . . . . . . . . . 62

4.2.2 DRIE: introduction of the big scallops along the sidewall. . . . . . . . . . . . . . . . . . . . . 62

4.2.3 Approach A: silicon nitride-based corner lithography 64

4.2.4 Approach B: combination of digital etching-based corner lithography with edge lithography . . . . . . 68

4.2 .5 Visualization . . . . . . . . . . . . . . . . . . . . . . . 69

4.3 Results and Discussion . . . . . . . . . . . . . . . . . . 69

4.3.1 Structure with highly isotropic big scallops . . . . . . 69

4.3.2 Approach A: silicon nitride-based corner lithography 70

4.3.3 Approach B: combination of digital etching-based corner lithography with edge lithography . . . . . . 73

4.4 Conclusion . . . . . . . . . . . . . . . . . . . . . . . . . 76 
5 A photocathode 79

5.1 Results . . . . . . . . . . . . . . . . . . . . . . . . 81

5.1.1 Spatioselective coating of Ni-Mo on microwires .. 81

5.1.2 Photoelectrical (J-V) measurements . . . . . . . . . 84

5.1.3 Photoelectrochemical measurements ........ . 91

5.2 Discussion . . . . . . . . . . . . . . . . . . . . . . 102

5.3 Methods . . . . . . . . . . . . . . . . . . . . 107

5.3.1 Fabrication of radial $n+/ p$ junctions in Si microwire arrays . . . . . . . . . . . . . . . . 107

5.3.2 LPCVD of silicon dioxide passivation layer . . . . . 112

5.3.3 Local removal of silicon dioxide from the tops of $\mathrm{Si}$ microwire arrays .............. . 113

5.3.4 Electrical contacts . . . . . . . . . . . . . . . . 115

5.3 .5 Catalyst deposition . . . . . . . . . . . . 117

5.3.6 J-V measurements . . . . . . . . . . . . . . . . . . 117

5.3 .7 J-E measurements . . . . . . . . . . . . . . . . 118

5.3.8 Light source and calibration . . . . . . . . . . 120

5.3 .9 Gas chromatography . . . . . . . . . . . . . . 120

6 A photoanode 123

6.1 Hematite synthesis routes . . . . . . . . . . . . . . . . . . . . 124

6.1.1 Electrophoretic deposition . . . . . . . . . . . . 124

6.1.2 Hydrothermal growth . . . . . . . . . . . . . . 126

6.1 .3 Reactive sputtering . . . . . . . . . . . . . . . . 129

6.2 Tandem with silicon . . . . . . . . . . . . . . . . . 135

6.2.1 Electrical contact and transparency evaluation .. 137

6.2 .2 Tandem devices . . . . . . . . . . . . . . . . . . . 140

6.3 Conclusion . . . . . . . . . . . . . . . . . . . . . . . . 142 
7 Solar-to-fuel device concept $\quad 145$

7.1 The device concept . . . . . . . . . . . . . . . . . . . . . . . 145

7.2 Materials and methods . . . . . . . . . . . . . . . . . . . 148

7.2.1 Electrolyzer membrane fabrication . . . . . . . . . . 148

7.2.2 Solar-assisted electrolyzer membrane fabrication . 153

7.2.3 i-V and stability measurements . . . . . . . . . . . 155

7.2.4 Solar-assisted i-V measurements . . . . . . . . . . . . . 157

7.2 .5 Gas separation measurements . . . . . . . . . . . . 157

7.3 Results and discussion . . . . . . . . . . . . . . . . . . . 159

7.3.1 Fabrication results . . . . . . . . . . . . . . . . . . . . 159

7.3.2 Catalyst and photoanode performance. . . . . . . 161

7.3.3 i-V and stability measurements . . . . . . . . . . 162

7.3 .4 Gas separation . . . . . . . . . . . . . . . . . . . . . 168

7.4 Conclusion . . . . . . . . . . . . . . . . . . . . . . 169

8 Outlook 171

8.1 Architectures . . . . . . . . . . . . . . . . . . . . . 172

8.2 Absorber stack . . . . . . . . . . . . . . . . . . . . . . 174

8.3 Length scales . . . . . . . . . . . . . . . . . . . . . . 175

8.4 Conclusion . . . . . . . . . . . . . . . . . . . . . 177

9 Societal considerations 179

9.1 Scope ... . . . . . . . . . . . . . . . . . . . . . . . . 179

9.2 Technical options and merits . . . . . . . . . . . . . . . 182

9.3 Economic considerations . . . . . . . . . . . . . . . . 185

9.4 Societal and political impacts . . . . . . . . . . . . . . . . 186

9.5 Conclusions and perspectives . . . . . . . . . . . . 187

$\begin{array}{ll}\text { Bibliography } & 217\end{array}$

$\begin{array}{ll}\text { Summary } & 219\end{array}$

8 
$\begin{array}{ll}\text { Samenvatting } & 223\end{array}$

$\begin{array}{ll}\text { Acknowledgments } & 227\end{array}$

$\begin{array}{ll}\text { Publications } & 231\end{array}$ 


\section{Chapter 1}

\section{Introduction}

Before you is a PhD thesis on the application of microfabrication techniques to the research field of solar water splitting. An important research field for the energy security of the future as you will see, especially in Chapter 9. One of the main propositions of this thesis is that the field of microfabrication can make a fruitful contribution to the development of technologies for using solar energy to synthesize fuels. This statement is appropriately supported by socio-economic considerations, performance comparisons, fabrication process flows, graphs, images, models and many words. But between those you will hopefully also read more. A longing for a clean world where every person can live in peace and prosperity. And also a little, perhaps, the personal story of a four-year PhD project.

\subsection{Solar water splitting}

The present fossil fuel-based economy is not future proof and needs radical transformation. One reason is that the combustion of these fuels leads to the emission of $\mathrm{CO}_{2}$, which is believed to be a major driving force for climate change ${ }^{l}$. This climate change in turn has a profound impact on the ecology of our planet ${ }^{2}$ and is therefore expected to significantly worsen living conditions for coming generations. Another reason for us not to base our future economy on fossil fuels is that they 
have a limited supply. Currently known reserves are expected to run out around $2050^{3}$. A final reason is a political one: many western states would prefer not to depend on non-western countries for their vital supply of energy.

Solar irradiation on earth exceeds current energy consumption by orders of magnitude ${ }^{4}$, and is therefore a promising alternative to fossil fuels. However due to the intermittent nature of this resource, it cannot be matched one-to-one with the present temporal electricity demand pattern. This means that the capacity of fossil fuel-based power plants currently has to be large enough to provide the full demand at night or during clouded days. If solar energy is to become the main energy source, a surplus has to be generated at the more sunny moments, and stored for use when it is dark or clouded. Water electrolysis to form oxygen and hydrogen is one $\mathrm{CO}_{2}$-neutral way to store the energy, in a chemical bond. The hydrogen can be stored, and later be recombined with oxygen in a fuel cell to retrieve (part of) the energy. Many other storage routes are considered, including those that somehow use solar energy to produce synthetic or plant-based fuels for use in existing power plants and vehicles.

Furthermore, if also transport of goods and persons is to be fueled by solar energy, the solar energy has to be brought on board of a vehicle. This sets limits to the energy density/specific energy of the storage method. The energy density and specific energy have to be high enough so that a reasonable action radius can be achieved without making the vehicle too heavy or too large. Hydrogen has a higher specific energy than fossil fuels, and if compressed, has a volumetric energy density between those of fossil fuels and battery storage, and is therefore an attractive energy carrier for vehicles that have to rely on solar energy.

Photovoltaic panels are currently applied at commercial scales to harvest solar energy, which is produced in the form of electricity. Also electricity is used to form hydrogen at commercial scale by alkaline and PEM electrolysis ${ }^{5}$. An integration of these two technologies into one device, photoelectrochemical (PEC) water splitting, is being researched with the hope that it will be cheaper and more efficient than the separate components. This thesis is meant as a contribution to this research field, and shows some examples of how microfabrication techniques can be applied in this field. 


\subsection{Microfabrication}

Microfabrication, also called micromachining, microelectronic fabrication or integrated circuit technology is the collection of technologies that are used for making for example integrated circuits and microelectromechanical structures (MEMS) on the micrometer and nanometer scale. Some examples are lithography, chemical vapor deposition, physical vapor deposition, (deep) reactive ion etching, wet etching and polishing. The materials used are mostly silicon, silicon oxide, silicon nitride, and several metals like aluminum, copper, tungsten, titanium and gold, although the integrated circuits of today contain almost the entire periodic table.

In solar water splitting devices, photons have to be absorbed to form charge carriers, which have to be separated and reach the electrodes at which they perform their respective half-reactions (oxygen evolution or hydrogen evolution), the reaction products have to be removed from the electrodes (and not be mixed, since these mixtures are highly explosive), and ions need to be transported from one electrode to the other to close the electrical circuit. All of these processes have their minimum, typical or maximum length scales, lest they become too inefficient for practical devices. Many of these typical lengths are on the order of the micrometer or even nanometer scale, and therefore it is expected (and indeed has been shown) that specific microstructures can help in improving efficiencies and mitigating tradeoffs between some of these processes. Microfabrication techniques can be used to produce devices which are structured on this small scale, but are produced in parallel by the millions to cover surface areas of at least square centimeters, the typical scale for lab experiments on solar water splitting devices. Scaling of some of these techniques to the millions of square kilometers that are needed to capture enough solar energy for the whole world population might be problematic. To achieve such structures at a much larger hydrogen production scale, alternative processes may become available by further development of processes such as metal-assisted chemical etching, vaporliquid-solid growth or laser ablation, to create the silicon microwires and microholes in silicon that are discussed in this thesis. 


\subsection{Thesis outline}

Chapter 2 starts with giving considerations that have to be taken into account when developing a device architecture for solar-to-hydrogen applications. Then, Chapters 3 and 4 report on microfabrication techniques that have been developed to achieve specific structures for solar water splitting devices, but might find application elsewhere and are therefore described rather independently from the application that is the main theme of this thesis. However Chapter 5 clearly shows that one of these techniques is a useful tool for studying the individual contributions of light absorption and catalysis in a microstructured photocathode.

Chapter 6 is centered around a much studied photoanode material: hematite, a specific crystal phase of iron(III) oxide. Several synthesis methods are considered, and it is shown how microfabrication techniques can be used to synthesize this material and increase its output voltage by integrating it with a silicon solar cell. Chapter 7 presents an innovative concept for a fully functional device made of earthabundant materials, that upon exposure to sunlight should produce separate oxygen and hydrogen streams. The experimental results presented show the promising nature of this concept. Chapter 8 gives expectations and recommendations for further technological development of solar water splitting devices, while Chapter 9 does the same for the societal, political and economic aspects of these developments. 


\section{Chapter 2}

\section{Performance considerations}

The price of solar hydrogen scales almost inversely with the efficiency of the conversion process from photons to hydrogen gas (see Chapter 9). There are many steps in this process, and if any of these steps is inefficient, this will influence the overall efficiency and with that the price of solar hydrogen. First of all, as many photons as possible have to reach the right semiconductor components of the device. There, as many photons as possible have to be absorbed in such a way that they excite an electron from the valence band to the conduction band, leaving a hole behind. As many of these holes and electrons have to reach separate electrodes, where again the amount of charge carriers performing the right water splitting half-reactions has to be maximized. The ions generated and consumed in these reactions have to be transported with small losses, and the gasses generated have to make up the vast majority of the separate product streams. All these steps are described in this Chapter, with the corresponding loss mechanisms. Also insights from literature are given, which could help to reduce certain losses or bypass apparent trade-offs between different losses.

\subsection{Light absorption}

The first step in the conversion from photons to hydrogen gas is the absorption of the photons by a semiconductor where they can excite 
electrons from the valence band to the conduction band. Several things can go wrong here: the photons are simply reflected from the surface of the device, they pass through without being absorbed, or they are absorbed by the wrong material.

\subsubsection{Reflection}

Light is most strongly reflected when a large jump in refractive index is encountered. For example, silicon has a refractive index of 4.3 at a wavelength of $500 \mathrm{~nm}$, and almost $40 \%$ of this light would be reflected from a flat silicon surface in air if no additional measures are taken. Reflection can be reduced by making the transition from air to silicon (or a different semiconductor) more gradual.

One way to do this is to add one or more transparent layers with a refractive index between that of air and of the semiconductor. If the thickness of these layers is chosen well, constructive and destructive interference can further contribute to enhanced light absorption. For solar cells this can be a dielectric layer like silicon oxide or silicon nitride. However for solar-to-fuel devices, if such an anti-reflective coating is not electrically conductive, it will prevent transfer of electrons to or from the solution, so no electrochemical reaction can be performed on the surface of the semiconductor in that case. Apart from that, if the charge separation in the semiconductor is dependent on band bending caused by the contact with the electrolyte (solid-liquid junction), such a coating layer would alter or prevent the band bending in the semiconductor, and might therefore interfere with the charge separation.

Another way to make the optical transition from air to the semiconductor more gradual, is to structure the surface of the semiconductor. Especially sub-wavelength features result in a reduced effective refractive index, because to the light the material looks like a mixture of air and the semiconductor. But also larger features can diffract the light so that less of it is reflected. These larger features are typically also necessary for proper charge separation, as discussed below in Section 2.2. In earlier work it was shown that arrays of micropillars with radial charge separation can increase solar cell photocurrents from $24 \mathrm{~mA} \mathrm{~cm}^{-2}$ for flat solar cells to $38 \mathrm{~mA} \mathrm{~cm}^{-2}$ for the pillar array cells ${ }^{6}$, without the use of antireflective coatings. The theoretical maximum photocurrent for silicon 
solar cells is $46.8 \mathrm{~mA} \mathrm{~cm}^{-2}$ (see Figure 5.35 , so by this approach the optical losses are reduced from $48 \%$ to $17 \%$ (assuming that the non-ideal photocurrent is completely caused by optical losses).

\subsubsection{Transmission}

If photons are to generate charge carriers in a semiconductor, their energy has to be higher than the band gap of that semiconductor. Semiconductors are, apart from absorption by defects, transparent to light with longer wavelengths. So in terms of light absorption, a lower band gap is always better. However the band gap of a semiconductor also has a direct influence on the maximum built-in electric potential difference at a p-n junction in that material, and therefore on the voltage that can be generated from that material. So in that sense, a higher band gap would be beneficial. This trade-off was analyzed (along with parasitic light absorption, see below) amongst others by Döscher et al. ${ }^{7}$. From their calculations it seems that a stack of two different semiconductors with band gaps around 1.0 and $1.6-1.8 \mathrm{eV}$ would be the most ideal combination to supply the full voltage needed for water splitting. Unfortunately in practice no earth-abundant semiconductor, except crystalline silicon, comes close to its predicted theoretical output voltage ${ }^{8}$, and this means that for practical devices one or both of the semiconductors need to have a higher band gap, so that they can actually produce the required voltage?

These calculations are always based on the assumption that all the photons with an energy above the band gap are absorbed by the material. However in practice only a certain portion of the light is absorbed by a certain amount of material, such that there is an exponential decay of light intensity inside the material. The strength of this decay is typically wavelength dependent, and usually especially the longer wavelength light decays more slowly. However if the material becomes too thick, proper charge separation with little recombination may become a problem. Therefore in practice the semiconductor will always have a limited thickness, and some of the light is transmitted through the material. This trade-off can be circumvented partially by structuring the semiconductor material and/or including a back side reflector in such a way that the average path length of the light inside the material becomes longer and more light is absorbed. 
Another reason why some of the light might be transmitted through the device is a device design where not all surface area is used by light-absorbing structures. An important reason to do this is to allow ion transport from one side to the other side of the device, as discussed below in Section 2.4. One device architecture in which this might occur is the one presented for example by Yahyaie et al. ${ }^{10}$. It consists of a membrane which conducts ions but separates gases, with on either side semiconductor rods which together provide the water splitting voltage. The earlier work on radially doped silicon micropillars in our group ${ }^{6}$ was also done with this architecture in mind: these micropillars could be the rods on one side of such a membrane. To better understand the enhanced light absorption of these structures, and assess their suitability for use in such a device, light absorption in such pillars was modeled numerically.

These simulations were performed using the Lumerical FDTD program. Hexagonally packed microwire arrays of intrinsic monocrystalline silicon were defined, as shown in Figure 2.1. The wires were made with a diameter of $4 \mu \mathrm{m}$, and a minimum distance of $4 \mu \mathrm{m}$. Due to the limited availability of computational power, pillars with a length of 5, 7 and $10 \mu \mathrm{m}$ were simulated, instead of the $40 \mu \mathrm{m}$ that was experimentally found to be optimal ${ }^{6}$, because of memory limitations. In this structure, a simulation domain was selected as shown in Figure 2.2, which extended $5 \mu \mathrm{m}$ into the flat substrate. There it is also shown that some symmetry boundary conditions were used to further reduce the simulation domain. Simulations were performed for wavelengths from 350 to $1100 \mathrm{~nm}$ in 75 steps, to include most of the solar spectrum with photon energies above the band gap of silicon. Local charge generation rates were calculated for two polarizations (normal incidence) and then locally averaged for all these wavelengths and polarizations, weighted by the relative light intensity of each wavelength in the AM $1.5 \mathrm{G}$ spectrum. Meshing was performed automatically by the software to have at least 10 mesh points per wavelength for every simulation. A coarser mesh (100 nm between mesh points) was defined manually for all positions more than $800 \mathrm{~nm}$ below the surface of the substrate, and in the pillars between $800 \mathrm{~nm}$ above the substrate and $1600 \mathrm{~nm}$ below the top of the pillar, to reduce memory requirements and calculation time. Top and bottom boundary conditions were perfectly matched layers (PML), and the side boundary conditions were adjusted to the polarization: symmetric for the boundaries parallel to the polarization, and 


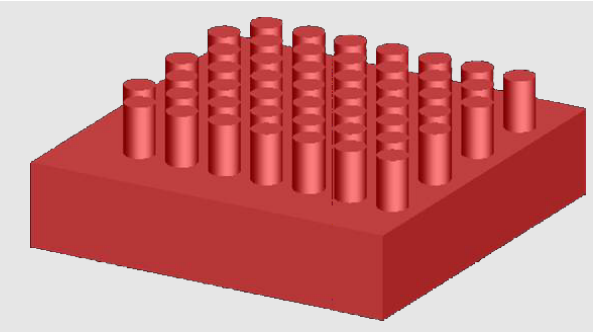

Figure 2.1: Silicon microwire array defined in Lumerical

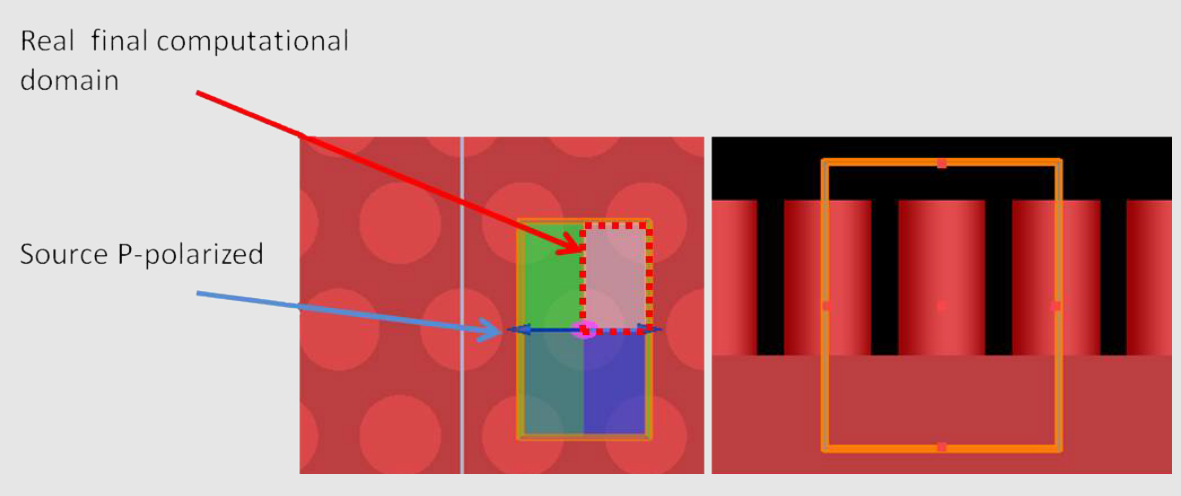

Figure 2.2: Simulation domain for the optical simulation of microwires

anti-symmetric for the boundaries perpendicular to the polarization.

The results of the numerical simulations for $10 \mu \mathrm{m}$ long pillars are shown in Figure 2.3. Strikingly, a $34 \%$ of the light absorption takes place in the substrate, and light that enters the pillars is mostly absorbed at their tops and centers, hardly at their sidewalls. Although longer pillars were not simulated, it is clear that device architectures that would not have such a substrate present, like the one presented by Yahyaie et al. ${ }^{10}$, would transmit a large part of the light that enters under normal incidence. 


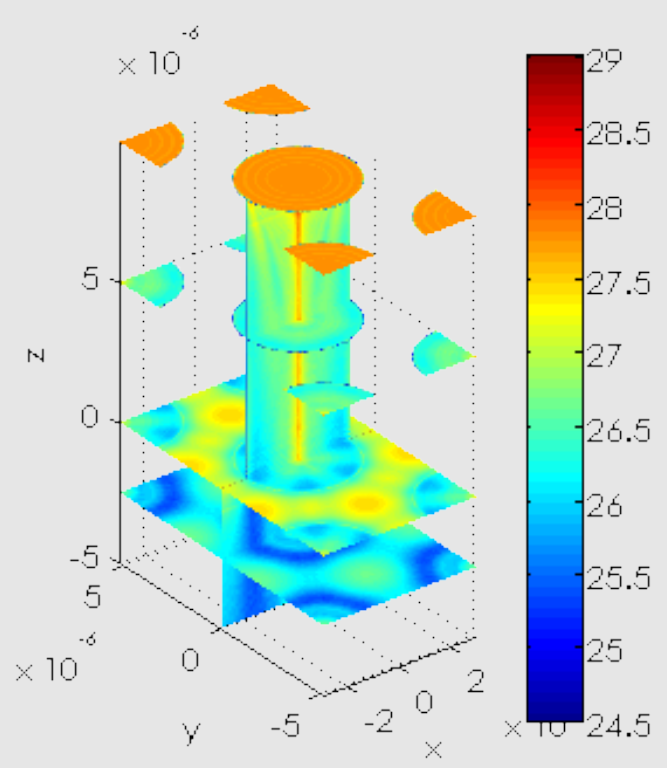

Figure 2.3: Numerically calculated charge carrier generation rates resulting from AM $1.5 \mathrm{G}$ solar illumination of $10 \mu \mathrm{m}$ long silicon micropillars $\left(\log _{10}\right.$ of electron-hole pairs $\mathrm{cm}^{-3} \mathrm{~s}^{-1}$ ). The substrate is below $z=0$.

\subsubsection{Parasitic light absorption}

A photoelectrochemical cell contains many materials that are not intended for light absorption, but are nevertheless not completely transparent. In many device concepts, light would first pass through for example the electrolyte, separation membrane and/or catalysts before it reaches the semiconductor light absorbers. A discussion of device architectures can be found in Section 8.1.

Döscher et al. ${ }^{7}$ have analyzed the effect of light absorption by the electrolyte theoretically, and have shown that already for a water film of $1 \mathrm{~cm}$ thick, around $10 \%$ of the light of relevant wavelengths is lost.

In some device architectures, a gas separation membrane could be positioned between (part of) the light absorber and the sun, causing some parasitic absorption. However typical polymer ion exchange 
membranes like Nafion ${ }^{\circledR}$ are quite transparent $(94.6 \%$ transmittance for a $150 \mu \mathrm{m}$ thick membrane ${ }^{11}$ ).

A bigger problem is probably light absorption and reflection by electrocatalysts. Again this is only an issue if the light passes through the electrolyte before it reaches the semiconductor(s). Especially earthabundant catalyst materials typically need high mass loadings to reach low overpotentials. This in turn leads to a high amount of parasitic light absorption, especially for hydrogen-evolution catalysts, which are typically metals, as compared to oxygen-evolution catalysts, which are typically metal oxides or hydroxides, which are usually more transparent (but can still absorb a significant part of the incident light). A way to bypass this trade-off is presented in Chapter 5.

Another source of parasitic light absorption could be the connection layers which are needed to stack multiple semiconductor junctions and achieve ohmic contacts between them. This typically requires layers with metallic conduction, and since light is an electromagnetic wave it is strongly absorbed by highly conductive materials. There is always a trade-off between conductivity (to achieve lower ohmic losses) and transparency of these layers. However some materials, like transparent conductive oxides (TCOs) are quite optimal in this trade-off.

\subsection{Charge separation}

Useful light absorption in semiconductors occurs when a photon transfers its energy to an electron in the valence band, and the energy is high enough so that the energy of the electron becomes at least as high as the energy level of the conduction band of the semiconductor. Subsequently, any surplus energy is released as a phonon, and the electron falls back to the conduction band (thermalization). This process leaves a hole (a spot where there should normally be an electron) in the valence band. However the surplus energy released by thermalization does not contribute to the water splitting and is lost. If there is a built-in gradient of free electrons in the semiconductor, caused by doping or by contact with a different material (semiconductor, metal or electrolyte), the two opposite charges that were formed will start to move in opposite directions. The effectiveness of this separation process depends on several variables: the mobility of the charge carriers 
in the material, the steepness of the built-in gradient, and the lifetime of the charge carriers. Mobility is an intrinsic property of the semiconductor material used. A good gradient requires careful tuning of doping levels and the material combinations and thicknesses. It is also important that this gradient is in the right direction, otherwise it will deteriorate charge separation instead of facilitating it.

The charge carriers have a finite lifetime: electrons can fall back to holes in the valence band, where they recombine, releasing their energy in the form of heat or light. Recombination is facilitated by defects, like impurities (doping atoms for example) and crystal defects (like dislocations or grain boundaries). So higher purity and crystallinity improve charge carrier lifetimes and therefore charge separation. Also interfaces typically promote recombination because they contain a lot of defects like crystal lattice termination and dangling bonds. Proper passivation layers can reduce this effect by reacting with the dangling bonds, as well as built-in electric fields that repel one type of charge carrier (recombination can only occur if both types are present).

Finally electrons have to leave the semiconductor on one side, and holes have to be filled with electrons on the other side of the material to generate a current. This requires a proper ohmic contact to the semiconductor to avoid an extra, unwanted voltage drop during operation. This is facilitated by a high local doping level, and alignment of the electron energy levels in both materials (semiconductor: Fermi level; metal: work function; electrolyte: reaction equilibrium potential).

\subsection{Catalysis}

Once charge carriers have reached the interface with the electrolyte, the electrochemical half-reaction has to be performed, by transferring one or more electrons to or from molecules in the solution or adsorbed at the surface. This is facilitated by electrocatalysts that have a suitable binding energy for reaction intermediates. The activity of the catalyst is intrinsic to the local electronic structure of the catalyst material. The activity can depend on crystal plane, and can often be tuned by alloying multiple materials. In general, exposing more catalyst surface to the electrolyte improves the catalyst performance. This can be done 
by structuring, and if the conductivity of the catalyst is low or the material is expensive, a conductive support matrix can facilitate this. As long as not the whole surface is covered, or if the catalyst is porous, simply putting more catalyst material also results in a larger catalytic surface area. However, this may also have the effect that the catalyst blocks more light for the underlying semiconductor.

Catalysts can be positioned strategically to reduce parasitic light absorption or to reduce the length of the transport path for the reactants or reaction products.

As long as mass transport is not limiting, the reaction speed usually increases exponentially with the potential difference between the charge carriers and the equilibrium potential of the electrochemical half-reaction, as in the Tafel and Butler-Volmer relations between reaction rate and overpotential. So applying a higher potential difference between the anode and cathode speeds up the reaction rate. However it also leads to higher losses: the amount of energy stored in chemical bonds only scales with the passed charge, while the input power also scales with the applied voltage, which should therefore be kept as low as possible.

Some semiconductors may have suitable band gaps for a visible light-absorbing tandem device, but it could be that their band energy levels are not properly aligned to the equilibrium potentials of the half-reactions that are to be performed. This energy difference is what determines the overpotential for the reaction. If it is too low, charge carriers may not be exchanged quickly enough with the electrolyte, and they will accumulate at the surface, where they may recombine before performing the reaction for which they were intended. If the energy difference is very high, the reaction may be driven at a very high rate, but the energy losses are also higher. This means that the semiconductor is providing more voltage than needed, and that its band gap could also have been lower so that it could have absorbed more light and therefore produced a higher current. The energy levels of the semiconductor and half-reaction can be decoupled by making ohmic contacts to the semiconductor. However, in that case there has to be a built-in p-n junction for the charge separation, since the semiconductor-liquid junction can no longer be used. In that case the semiconductor p-n junction turns into a 'floating' power source with a certain current-voltage relation that holds regardless of 
the half-reactions performed and the energy levels of the semiconductor bands.

\subsection{Ion transport}

Depending on the $\mathrm{pH}$ of the electrolyte, protons are produced at the anode and consumed at the cathode (acidic electrolyte), or hydroxide ions are produced at the cathode and consumed at the anode (alkaline electrolyte). If there would be no ionic transport between the anode and cathode, there would be a quick buildup of charge and change in $\mathrm{pH}$ at both electrodes, so that the current flow is stopped. The $\mathrm{pH}$ can be held more constant by using a buffer solution or a strong acid or base. However charge transport is still necessary, so there has to be an electrolyte (liquid, polymer or solid) connection between the two electrodes. In the case of acidic electrolyte, the majority of the charge would be transported by protons, and this transport would also counteract the buildup of a pH gradient between the two electrodes. The argument is similar for alkaline electrolytes, where the charge transport would occur mainly through hydroxide ions.

For an acidic buffer $\mathrm{HA} \rightleftharpoons \mathrm{H}^{+}+\mathrm{A}^{-}$, there would be a buildup of $\mathrm{HA}$ at the anode and of $\mathrm{A}^{-}$at the cathode (under the condition that HA does not undergo a chemical reaction at the anode), and this gradient has to be counteracted by diffusion and migration of both species between the electrodes. In addition to this, migration and diffusion of protons will also occur. For an alkaline buffer, analogous effects will play a role. However, the use of a buffer prohibits the use of an ionselective or polymer electrolyte membrane for gas separation, since it would prohibit the transport of the neutral buffer species and either the protons or the conjugated base ions ${ }^{12}$. This could be overcome by recirculating the electrolyte between the compartments ${ }^{13}$, but that would lead to a large crossover of the reaction products and therefore explosive product streams, except maybe if a way can be found to efficiently degas the electrolyte before recirculation. Therefore a strong base or a strong acid is probably a more suitable electrolyte, but that puts more severe constraints on the choice and protection of the materials used in the device. For example, there is currently no acid-stable earth-abundant oxygen evolution catalys ${ }^{14}$. So for low ion transport losses, a strong base is probably the best electrolyte. 
However, even a strongly alkaline solution has a limited conductivity. Therefore a short conduction path from the cathode to the anode is important for reducing ion transport losses. Reece et al. ${ }^{15}$ already noticed that a wired configuration performs better than a wireless configuration. In a wired configuration, the electrolyte is sandwiched between a cathode and a photoanode, and the ions only have to cross the thickness of the electrolyte layer. In a wireless configuration, the anode and cathode are integrated on either side of the photoabsorber stack, which is completely immersed in the electrolyte, so that the ions have to travel all the way around the sample. Another disadvantage of such an approach is that there is always empty space needed around the device to facilitate ion transport, and this space cannot be used for light absorption. An idea to overcome this was introduced by Walczak et al. ${ }^{16}$ : several 'wireless' solar water splitters are placed in a louvered configuration, so that the ion-conductive membranes that separate the compartment can be placed parallel to the incident light, and as such do not reduce the area available for light absorption. Their modeling also showed that for $16 \mathrm{~mm}$ wide devices with $3 \mathrm{~mm}$ high Nafion ${ }^{\circledR}$ membranes in between, the ion transport losses are less than $5 \mathrm{mV}$ at the operating average current density of $0.39 \mathrm{~mA} \mathrm{~cm}^{-2}$ in $1 \mathrm{M} \mathrm{HClO}_{4}$, even though the ions had to travel around the complete device. Of course for higher current densities in more efficient devices, the distance between membranes would have to be reduced further to keep the ion transport losses limited.

In a way, this was achieved by the device architecture presented by Bosserez et al. ${ }^{17}$, although no light absorption was involved. From the ion transport perspective their device functioned like a wireless device, but it was perforated with numerous holes to allow ion transport between the front and back sides of the device. They found that decreasing the distance between the holes greatly reduced the ion transport overpotential of their devices, even though they kept the porosity (portion of the surface area used by the holes) more or less constant around 5 to $15 \%$ by going from a few big holes to many small holes. This suggests that by going to even more and smaller holes, the transport overpotential can be made negligible even if only a few percent of the surface area is used by the holes, and the remainder is available for light absorption. This was further investigated using simulations by Trompoukis et al. ${ }^{18}$. They also found esperimentally that these small perforations had only a minor effect on the performance of silicon mul- 
tijunction solar cells. In Chapter 7 we have experimentally investigated the ion transport behavior of such microporous membranes.

Hankin et al. ${ }^{19}$ used a perforated photoelectrode, but found little improvement compared to the device where the ions had to travel around a continuous $10 \cdot 10 \mathrm{~cm}^{2}$ photoelectrode. However because of the poor performance of the hematite photoelectrode they used, much less ions had to be transported than in the case of Bosserez et al. ${ }^{17}$, and this can also explain their low transport overpotentials.

\subsection{Gas crossover}

As discussed in Section 2.4, there has to be good ion conductivity between the anode and cathode in water splitting devices. However at the same time the mixing of the gases produced at these electrodes has to be prevented. One reason is safety: at 1 bar more than 4.0 mole percent of hydrogen in pure oxygen, or 4.8 mole percent oxygen in pure hydrogen would form an explosive mixture ${ }^{20}$. Another reason is efficiency: oxygen crossing over from the anode to the cathode could be reduced at the cathode instead of the water/protons, so that not all current is used for water splitting anymore. A similar argument holds for hydrogen crossing over to the anode. Both effects would lead to efficiency losses since some of the current is used for recombination of the reaction products instead of formation of these products.

In commercial electrolyzers, the gases are kept separated by membranes. One typical type of electrolyzer is the proton exchange membrane electrolyzer. Because the membrane in this case can only transport protons, gases are separated almost perfectly in these devices. Another type is the alkaline electrolyzer, which typically uses a porous membrane and a highly alkaline (and therefore conductive) electrolyte. This type is deployed commercially on a larger scale ${ }^{21}$. Because of the small pores, it is not energetically favorable for gas bubbles to move into the membrane. However dissolved gases can still diffuse through the electrolyte inside the pores, and therefore the crossover is higher. However, since these devices are operated at high current densities, the crossover is negligible compared to the gas production, and the adverse effects of crossover described above are of no concern. 
Both approaches could also be used for photoelectrochemical water splitting devices. However, care has to be taken that the membrane does not interfere too much with the light absorption. Also, the demands for separation performance are higher, since typically smaller current densities are expected, down to a current density of zero when the sun is not shining. In a way, Bosserez et al. ${ }^{17}$ used a porous membrane as a separator. However they found 17 to $24 \%$ of the gas in the oxygen compartment to be hydrogen, quite independently of the used pore size.

We also would like to point out that the gas bubbles can be prevented from reaching the wrong compartment without the use of a membrane. Buoyancy-driven bubble movement ${ }^{22}$ or electrolyte flow ${ }^{23}$ can direct the bubbles to the right collection compartment.

\subsection{Conclusion}

The many ways in which part of the energy can be lost in the conversion process from solar photons and water to hydrogen were discussed, and it was shown that many of these loss mechanisms cannot be addressed individually. Taking certain measures to reduce one of the losses may increase one of the other losses. The challenge is to find device architectures that can circumvent these trade-offs as much as possible, especially by finding the right combination and arrangement of semiconductor materials, making sure that catalysts and gas separators do not interfere with light absorption, and taking care of good ion conduction without reducing the illuminated semiconductor area or creating explosive product streams. A few architectures that attempt to address these challenges are discussed in Section 8.1, including the device concept that is investigated in Chapter 7. 


\section{Chapter 3}

\section{Microfabrication techniques for patterning of vertical silicon structures}

This chapter is based on "Sidewall patterning - a new wafer-scale method for accurate patterning of vertical silicon structures", Westerik et al. ${ }^{24}$.

As discussed in Chapter 2, large arrays of micro or nanopillars are considered for use in solar water splitting devices. However at the same time it became clear that the precise arrangement of semiconductors, catalysts and membranes can make the difference for the performance of the device, and innovative architectures can avoid tradeoffs between certain loss mechanisms. In this Chapter microfabrication techniques are developed to gain control over the location of materials at pillars that have dimensions in the micrometer range, but are spread out over large surfaces. The presented techniques also show promise for application in other fields. 
a.

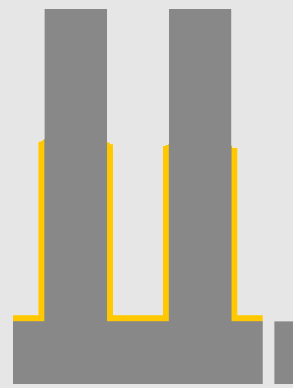

b.

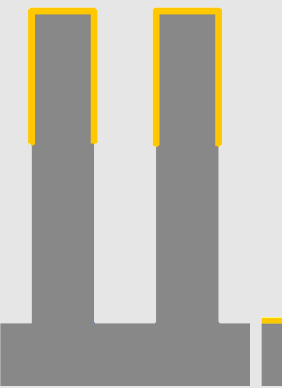

C.

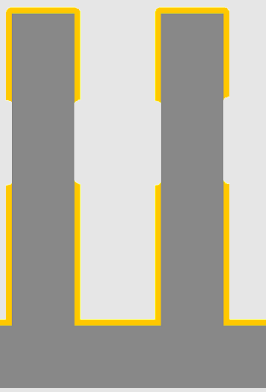

Figure 3.1: Sschematic representation of structures that can be obtained applying the techniques described in this Chapter: silicon (gray) micro-/nanostructures covered by a conformal material (yellow), where selectively the top part (a), bottom part (b) or a band halfway (c) is opened to expose the underlying silicon. Note that this picture is not to scale - in principle the size and aspect ratio of the structures can be varied, and also the height(s) of the transition between the covered and exposed parts can be changed in a well-controlled way.

\subsection{Comparison to other 3D microfabrication techniques}

Micro and nanofabrication are mostly achieved on flat (single-crystal silicon) substrates using techniques that modify the substrate as a whole. In the out-of-plane dimension ( $z$ ), most of the structural and material contrast is obtained by various combinations of stacking and removal of films. Control of the in-plane dimensions ( $x$ and $y$ ) is typically obtained through lithography. By a combination of these two basic tools, complex 3D structures can be obtained, as can be seen for example in the complex 3D metal networks in integrated circuits. However, these are never truly 3D because the height of the structures that can be obtained is limited to a few micrometers because of the film growth rates of nanotechnology-compatible deposition methods. This is partly resolved by bulk micromachining (etching into the substrate), but here the shape control in the $z$-direction is limited by the etching mecha- 
nism (isotropic, directional, or determined by crystallographic directions). The goal of this Chapter is to demonstrate routes for accurate out-of-plane 'lithography', to obtain the 3D structures as those shown in Figure 3.1.

The ultimate technologies for obtaining similar control over all three dimensions of structures probably come from the field of 3D printing/writing, in which material is deposited or modified only at the exact position where it is desired. Some of these techniques reach the micrometer or even nanometer scale $25-31$, especially two-photon polymerization laser writing. Many complex structures can be realized with this technique, as long as every voxel is attached to another voxel or the substrate. The main disadvantage of this technology is the throughput. The technology is inherently slow, since the structures are created in a serial manner, while the more conventional thin-film and lithographybased micro-/nanofabrication technologies can produce many structures in parallel on a single wafer or even on multiple wafers simultaneously.

Some interesting wafer scale alternatives can be found in more advanced lithography that uses photoresist as a 3D material instead of only as a 2D film. Grayscale lithography can achieve local gradients in photoresist thickness that can be amplified in the material underneath with a factor $F$, if a directional etching process is used that has etch rates $\epsilon_{\text {resist }}$ and $\epsilon_{\text {material }}=F \epsilon_{\text {resist }} 31,32$. Nano imprint lithography (NIL) can also be make use of a 3D stamp to create height differences in the resist (multilevel NIL) ${ }^{31,33}$, and the same amplification trick can be used. Laser interference lithography can be used in three dimensions ${ }^{34}$, but is limited to periodic structures.

Similar structuring as can be achieved by grayscale lithography can also be achieved by directly controlling the sidewall angle during deep reactive ion etching (by changing the etch parameters as a function of etch time), and this also allows the creation of either positively or negatively tapered sidewalls, or combinations thereof in a single structure ${ }^{35-40}$.

A completely different approach is to create structures bottom-up instead of top-down. Vapor-liquid-solid growth and templated electrodeposition can be used to grow wires, and the reactant gases/electrolytes can be changed during the process to obtain changes in material composition along the wires ${ }^{41-49}$. Further processing of such wires 
after the growth then also allows for changes in the radial direction ${ }^{50}$. Self-assembly is another bottom-up strategy to achieve 3D structures at this small scale ${ }^{51}$. Also, bottom-up grown carbon nanotubes can be further processed to connect ${ }^{52}$ or locally modify ${ }^{53}$ these nanotubes to create 3D structures.

Some specific 3D structures can sometimes be obtained by applying conventional wafer-scale nanofabrication techniques in unconventional combinations ${ }^{31,54-72}$. Many of these use the edge or corner of some feature as a starting point for defining new features ('Edge Lithography' 57 or 'Corner Lithography' 60 , see also Chapter 4) and/or the specific orientation of certain surfaces which results in a contrast in deposition or etching speed.

For patterning regular arrays of wires or pores with micrometer or nanometer dimensions in the vertical direction, a rather straightforward technique exists. The spaces between the wires or the holes themselves are filled with polymer (or with a conformally grown layer of some other material) which is subsequently etched back to a certain height to facilitate the different treatment of the top and bottom of these wires or pores ${ }^{73-81}$ (see also Section 5.3.3). However such methods are prone to air trapping and non-uniformity, and usually require adjustment of the polymer deposition procedure for every individual geometry (diameter, pitch, height, etc.). With the use of a completely filling conformal layer with good deposition uniformity, the air trapping and nonuniformity can be avoided, but for larger wire spacings or hole diameters prohibitive layer thicknesses are required for complete filling.

Here we report on wafer scale techniques that achieve accurate out-of-plane patterning, to obtain structures such as those shown in Figure 3.1. This enables high throughput fabrication of complex micro and nanostructures with potential applications in many fields. In order to develop a versatile, accurate and reproducible patterning method for the sidewalls of out of plane silicon structures, (Retraction) Edge Lithography and standard wafer-scale technologies such as chemical vapor deposition, thermal oxidation and selective wet etching (which are intrinsically $3 \mathrm{D}$ because of their isotropic/conformal nature) are combined with a newly developed surface orientation dependent etching technique. This technique resembles the sidewall spacer technology used in the integrated circuit industry, except that because an inclined ion beam is used for etching, lower lying horizontal structures can also be retained. The presented techniques give good control over the re- 
sulting structures, have good uniformity, are scalable and are quite insensitive to the shape and sidewall roughness of the starting structures of the silicon on which the patterning is applied.

In this Chapter, in separate sections for each of the structures of Figure 3.1, the fabrication processes and results are discussed, and an example of an application is presented. For all these processes it is crucial that the top surface of microstructures can be opened selectively, and therefore the novel technique that was developed to achieve this is discussed separately in Section 3.3.

\subsection{Modify the top part of a structure}

This section describes the technique by which the top part of a silicon micro-/nanostructure can be exposed selectively, while keeping the bottom part covered in silicon oxide, as shown in Figure 3.1a.

\subsubsection{Fabrication}

The fabrication procedure is shown schematically in Figure 3.2 and summarized in the caption of that figure (see Section 3.2.2 for more details). This section elaborates on the materials and methods that were chosen for the process. For a detailed discussion of the technique used in step d as shown in Figure 3.2, see Section 3.3.

The combination of silicon oxide, silicon nitride and (poly)silicon was chosen because these materials can be applied conformally by means of low pressure chemical vapor deposition, and because for each material a wet etching solution is available which etches the other two materials much slower, such that each material can be selectively etched without damaging the other materials too much.

The thermally grown silicon oxide layer was chosen as the outer layer because of the high quality of the layer (no pinholes) and its wellknown angle dependent etching rate in the ion beam etching system used (see Section 3.3).

In this material system, the etching solution for polysilicon, tetramethylamonium hydroxide (TMAH), has the highest etching rate and the 


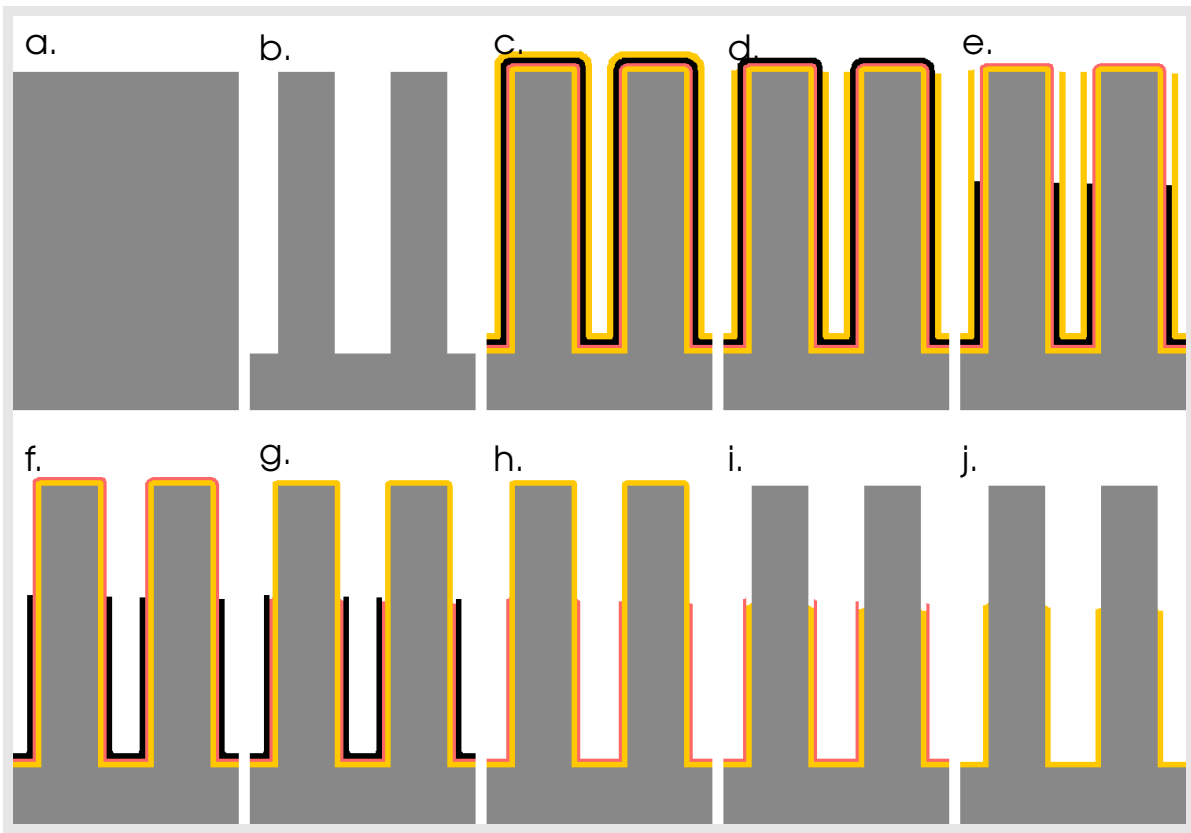

Figure 3.2: Sschematic process outline for opening only the top part of silicon structures ( $\square$ silicon substrate, silicon oxide, $\square$ silicon nitride, - polysilicon). (a) Silicon wafer, (b) deep reactive ion etching of patterned wafer to create vertical sidewalls, (c) conformal coverage of all structures with subsequently silicon oxide, silicon nitride, polysilicon and silicon oxide, (d) removal of silicon oxide from the top by inclined ion beam etching (see Section 3.3), (e) isotropic etching of polysilicon to the desired height, $(f)$ isotropic etching of silicon oxide, $(g)$ isotropic etching of silicon nitride, $(h)$ isotropic etching of polysilicon, (i) isotropic etching of silicon oxide and $(j)$ isotropic etching of silicon nitride. See text for a more detailed description. 


\begin{tabular}{l|r|r|r} 
& $\mathrm{TMAH}$ & $\mathrm{H}_{3} \mathrm{PO}_{4}$ & $\mathrm{BHF}$ \\
\hline polycrystalline silicon & 510 & 0.69 & $<0.1$ \\
thermal $\mathrm{SiO}_{2}$ & $<0.1$ & 0.59 & 65 \\
silicon rich nitride & 0.01 & 4 & $<0.1$
\end{tabular}

Table 3.1: Etch speed $\left(\mathrm{nm} \mathrm{min}{ }^{-1}\right)$ of the used materials in the used wet etching solutions

highest selectivity (see Table 3.1), and the etch rate is fairly constant even when etching deep down in narrow spaces. Therefore, the etching of polysilicon was chosen for the 'retraction' step (step e as shown in Figure 3.2): the achieved height can be predicted by linear extrapolation and the layers that encapsulate this retracting layer remain intact during the retraction. Furthermore, making use of the controlled retraction of a sacrificial layer for defining a mask edge minimizes the sensitivity of this technique to variations in sidewall angle as are encountered for example on structures with inclined or rough sidewalls.

The two layers below the polysilicon layer are needed to transfer the pattern formed in the polysilicon step by step to the underlying silicon structure. If they were not present, it would not be possible to remove this polysilicon hard mask without damaging the underlying silicon structure.

In principle, the procedure could be very much simplified if only two layers were used: first silicon oxide and then silicon nitride. The silicon nitride could be removed selectively from the top using inclined ion beam etching. Then the silicon oxide could be retracted to the desired height, and as a final step the silicon nitride could be etched selectively. However, the HF-solution that would be used for retracting the silicon oxide has only a limited selectivity especially towards silicon nitride, and the silicon nitride might fail after some retraction time. Also, the etch rate for the silicon oxide in HF-solution is typically not constant when etching in narrow trenches ${ }^{82}$.

In principle, the particular materials and methods chosen here are not essential to the approach described in this Chapter, but the properties described in this section are. Without these, the procedure would not work, or at least not as well and reliably as described here. 


\subsubsection{Fabrication details}

This section gives a detailed description of the steps that are summarized in Figure 3.2.

(a) The starting point is a silicon wafer (p-type, resistivity $5-10 \Omega \mathrm{cm}$, single side polished, OKMETIC), which is cleaned in room temperature $99 \%$ nitric acid and $95{ }^{\circ} \mathrm{C} 69 \%$ nitric acid subsequently, and thoroughly rinsed in DI water. (b) A $200 \mathrm{~nm}$ silicon oxide hard mask is formed by wet thermal oxidation at $1000^{\circ} \mathrm{C}$ for $20 \mathrm{~min}$. A pattern is applied using Olin OIR 907-17 positive resist and optical lithography. This pattern is transfered to the silicon oxide by RIE (Adixen with substrate temperature $-10^{\circ} \mathrm{C}, 20 \mathrm{SCCM} \mathrm{C}_{4} \mathrm{~F}_{8}, 15 \mathrm{SCCM} \mathrm{CH} \mathrm{CH}_{4}, 150 \mathrm{SCCM} \mathrm{He}$, pressure $8.5 \times 10^{-3} \mathrm{mbar}$, ICP power $2800 \mathrm{~W}$ and CCP power $350 \mathrm{~W}$ for $30 \mathrm{~s}$ ). This hard mask is then used to etch approximately $40 \mu \mathrm{m}$ straight down in the silicon (SPTS Pegasus, Bosch recipe with 133 cycles, deposition: $0.6 \mathrm{~s}$ with $150 \mathrm{SCCM} \mathrm{C} \mathrm{F}_{8}, \mathrm{ICP}$ power $2000 \mathrm{~W}, 20 \mathrm{mTorr}$; etching: $1.75 \mathrm{~s}$ with 275 SCCM SF$_{6}$, ICP power $2200 \mathrm{~W}$, CCP power increasing linearly from $20 \mathrm{~W}$ at the first cycle to $40 \mathrm{~W}$ in the last cycle, always with a $20 \%$ duty cycle, $26 \mathrm{mT}$ Torr; the wafer is kept at $20^{\circ} \mathrm{C}$ ). The photoresist and fluorocarbons are stripped (TePla 360 barrel etcher; step 1, preheat: $10 \mathrm{~min}$, 600 SCCM Ar, $0.6 \mathrm{mbar}, 1000 \mathrm{~W}$; step 2, resist strip: $10 \mathrm{~min}, 250 \mathrm{SCCM} \mathrm{O}_{2}$, $0.5 \mathrm{mbar}, 800 \mathrm{~W}$; step 3, fluorcarbon strip: $1 \mathrm{~min}, 237 \mathrm{SCCM} \mathrm{O}_{2}, 237 \mathrm{SCCM}$ $\mathrm{CF}_{4}, 0.5 \mathrm{mbar}, 80 \mathrm{~W}$; step 4, residual fluorcarbon removal: $1 \mathrm{~min}, 250 \mathrm{SCCM}$ $\mathrm{O}_{2}, 0.5$ mbar, $800 \mathrm{~W}$ ), followed by RCA-2 cleaning to remove contaminations from the plasma etching machines.

(c) Then, after cleaning again in the different nitric acid baths, followed by 2 minutes etching in $50 \%$ HF solution to remove the silicon oxide hard mask, several conformal layers are formed by LPCVD on the silicon structures: $100 \mathrm{~nm}$ of silicon oxide ( $400 \mathrm{mTorr}, 40$ SCCM of TEOS and $30 \mathrm{SCCM}$ of $\mathrm{N}_{2}$ at $725^{\circ} \mathrm{C}, 10 \mathrm{~min}$ ), $50 \mathrm{~nm}$ of silicon rich nitride (150 mTorr, $77.5 \mathrm{SCCM}$ of $\mathrm{SiH}_{2} \mathrm{Cl}_{2}, 20 \mathrm{SCCM}$ of $\mathrm{NH}_{3}$ and $150 \mathrm{SCCM}$ of $\mathrm{N}_{2}$ at $850^{\circ} \mathrm{C}, 12$ $\mathrm{min}$ ) and $170 \mathrm{~nm}$ of polysilicon (250 mTorr, $50 \mathrm{SCCM}$ of $\mathrm{SiH}_{4}$ and $250 \mathrm{SCCM}$ of $\mathrm{N}_{2}$ at $590^{\circ} \mathrm{C}, 50 \mathrm{~min}$ ). The outer $70 \mathrm{~nm}$ or so of polysilicon are then converted to about $135 \mathrm{~nm}$ of silicon oxide by wet thermal oxidation ( $45 \mathrm{~min}$, $900^{\circ} \mathrm{C}$ ).

(d) The ion beam etching was performed in an Oxford lonfab 300Plus system (at $2.4 \times 10^{-4}$ Torr; neutralizer: $5 \mathrm{SCCM}$ argon, $100 \mathrm{~mA}$; RF plasma: $125 \mathrm{~W}$; beam: $5 \mathrm{SCCM}$ argon, $50 \mathrm{~mA}$, acceleration $300 \mathrm{~V}$; etching time: 6 to $10 \mathrm{~min}$ ) and the rate of silicon removal was monitored using SIMS. This 
rate is lower when etching silicon than when etching silicon dioxide, so a decrease in the silicon signal in the SIMS was taken as an indication that in some spots the silicon oxide was removed (almost) completely and the silicon surface was reached. The etching was stopped when the change in the silicon signal was completed for about $80 \%$. Subsequently the sample was etched for $5 \mathrm{~min}$ in $1 \%$ aqueous HF solution to make sure that the underlying polysilicon was really accessible for further etching steps.

(e) Retraction of polysilicon under the outer oxide layer is done in $25 \%$ aqueous tetramethylammonium hydroxide (TMAH) solution at $70^{\circ} \mathrm{C}$ to the height at which the difference between top and bottom needs to be made, in this case 20 and 60 min to show different heights. (f) The outer silicon oxide is removed by etching $210 \mathrm{~s}$ in $12.5 \% \mathrm{HF}$ in $\mathrm{NH}_{4} \mathrm{~F}$ ( $1: 7 \mathrm{BHF}$ ) and ( $\mathrm{g}$ ) the polysilicon pattern is transferred to the underlying silicon rich nitride by etching $25 \mathrm{~min}$ in $180^{\circ} \mathrm{C} 85 \%$ aqueous $\mathrm{H}_{3} \mathrm{PO}_{4}$ solution. (h) The polysilicon is removed by etching $1 \mathrm{~min}$ in the TMAH as described in step (e) (preceded by 1 min etching in $1 \%$ HF and rinsing), and (i) the pattern is transferred to the inner silicon oxide layer by etching $2 \mathrm{~min}$ in $\mathrm{BHF}$ as described above. (j) Finally the silicon rich nitride is removed by etching again $25 \mathrm{~min}$ in hot $\mathrm{H}_{3} \mathrm{PO}_{4}$ solution as described above.

\subsubsection{Results and discussion}

Figure 3.3(a) and (e) show that the process can be applied to silicon micropillars of various thicknesses and spacings, and that the edge of the silicon oxide can be controlled to be at various heights. Figure 3.3(d) shows that it can also be applied to microholes. Figure 3.3(b) shows that the transition is well-defined, even on rough sidewalls. Only for structures with very large open areas around the vertical structures was the lower part of the structures also uncovered (Figure 3.3(c)). This effect is explained in Section 3.3.

The distance of the transition location from the top of the structures was measured from the SEM pictures, and was related to the TMAH etching time (step e as shown in Figure 3.2). Assuming a linear relation, the etch speed for this step was found to be $510 \pm 50 \mathrm{~nm} \mathrm{~min}{ }^{-1}(1 \sigma)$, based on several structures from wafers etched for various times. On average, there was no significant offset in the linear fit, but for short 

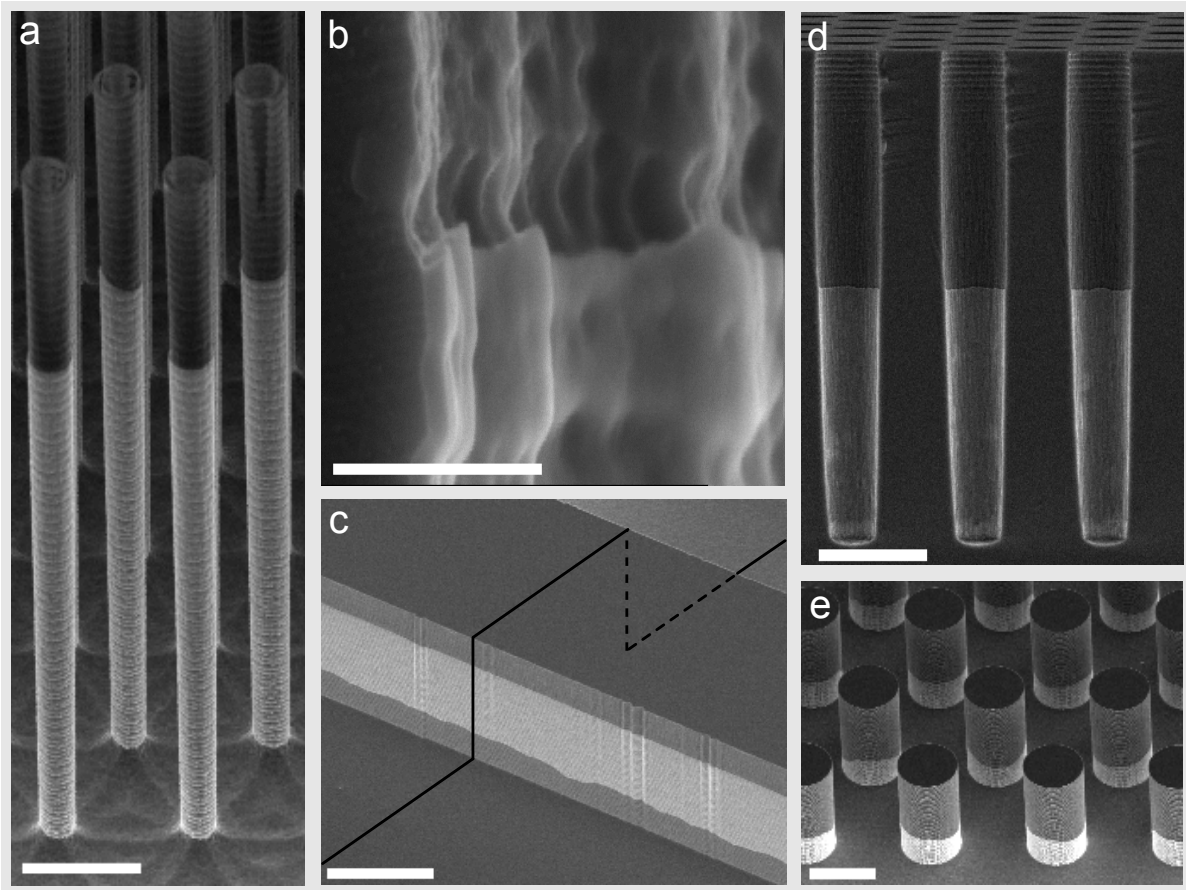

Figure 3.3: SEM images of various silicon structures of which the bottom part is covered with silicon oxide (lighter gray): (a) narrow micropillars, (b) close up of a sidewall at the transition, (c) bar structure surrounded by open space, with cross section indicated in black, where only a band of silicon oxide (lighter) has remained in the middle of the sidewall (d) microholes and (e) wide micropillars. Scale bars: (a) 5, (b) 0.5, (c) 25 , (d) 5 and (e) $25 \mu \mathrm{m}$.

and long etch durations standard deviations of respectively 1.5 and $1.6 \mu \mathrm{m}$ between the heights on various structures on the same wafer were found. Since the standard deviation hardly increases with etch time, the spread in values is probably caused by a geometry related startup effect rather than by a variability in the TMAH etching speed.

This process has been applied to achieve spatioselective passivation on a microstructured silicon photocathode for the photoelectrochemical reduction of water to hydrogen, as described in Chapter 5. 


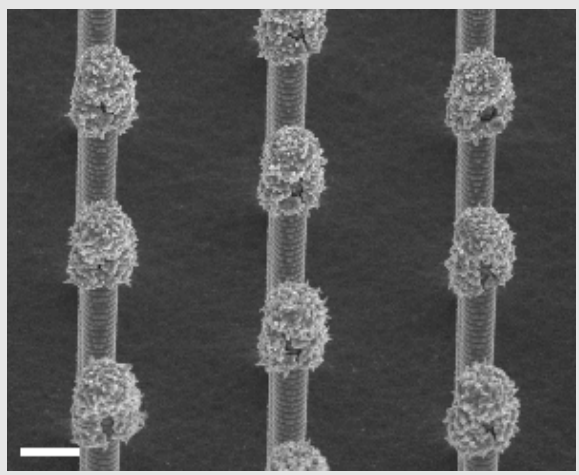

Figure 3.4: SEM image of silicon micropillars of which only the bottom part and the horizontal surfaces between the pillars was covered in silicon oxide, and subsequently the top was covered with catalyst material by electrodeposition. The scale bar is $5 \mu \mathrm{m}$.

The well-controlled patterning of the passivating oxide layer facilitated the localized electrodeposition of the nickel-molybdenum alloy catalyst, thereby preventing excessive light absorption without compromising the catalytic performance of these devices. An array of such micropillars that have catalyst material deposited on them only at their tops is shown in Figure 3.4.

\subsection{Selective opening of top surfaces}

An important step for the processes described in this Chapter is the selective etching of the top surfaces of structures. In short, this is done by inclined ion beam etching of a rotating sample. In this section, the principles and test results from this technique are described in more detail.

The selective etching of silicon oxide on the top surfaces is based on two principles. Firstly, the silicon structures cast shadows on everything between them: the ion beam does not reach all surfaces between the structures during the full rotation of the substrate. Secondly, the etch (or sputter) rate of silicon oxide depends on the angle of incidence of 
the ion beam. This dependence can be described by ${ }^{83}$ :

$$
R(\theta)=\left\{\begin{array}{cc}
R(0) \cos |\theta| e^{a \sin ^{2}|\theta|} \mid & |\theta|<90^{\circ} \\
0 & |\theta| \geq 90^{\circ}
\end{array},\right.
$$

where $R$ is the sputter speed, $\theta$ the angle of incidence of the ion beam (with respect to normal incidence, the angle with respect to the horizontal plane is $\left.\gamma=90^{\circ}-\theta\right), R(0)$ the sputter rate at normal incidence and $a$ a material dependent parameter. Etch rates for horizontal surfaces of thermally grown silicon oxide were measured for the used ion beam etching system (see Section 3.2.2) as a function of angle (circles in Figure 3.5), and equation (3.1) was found to fit the measured etch speeds best for $R(0)=7.41 \mathrm{~nm} \mathrm{~min}{ }^{-1}$ and $a=1.56$ (solid line in Figure 3.5).

Next, the etching speeds for horizontal and vertical surfaces are calculated as a function of the ion beam incident angle $\theta$ and the rotation of the substrate $\phi$. The symbols used are visualized in Figure 3.6. The incident angle on horizontal surfaces will always be equal to $\theta$, and is independent of $\phi$. Therefore, the etch speed of horizontal surfaces that are never in the shadow of a structure is described by equation (3.1) and represented by the solid curve in Figure 3.5. The incident angle on vertical surfaces, $\alpha$, is defined with respect to the plane of the vertical surface, and depends on both $\theta$ and $\phi$, as:

$$
\sin \alpha=\cos \phi \cos \gamma=\cos \phi \sin \theta .
$$

Substituting $90^{\circ}-\alpha$ from equation (3.2) for $\theta$ in equation (3.1) gives the etch rates for vertical surfaces that are not in the shadow of some other structure:

$$
R(\phi, \theta)=\left\{\begin{array}{cc}
R(0) \sin \theta \cos \phi & |\phi|<90^{\circ} \\
\cdot e^{a\left(1-\sin ^{2}(\theta) \cos ^{2}(\phi)\right)} & |\phi| \geq 90^{\circ} \\
0 &
\end{array} .\right.
$$

Averaging this etch speed over a full rotation of $\phi$ gives the average etch speed of vertical surfaces as shown by the dashed curve in Figure 3.5. The ratio between the two etch rates is shown in Figure 3.5 by the dash-dotted line.

These graphs can be understood as follows. For small angles, the ion beam is (almost) parallel to vertical surfaces, so they will hardly be 


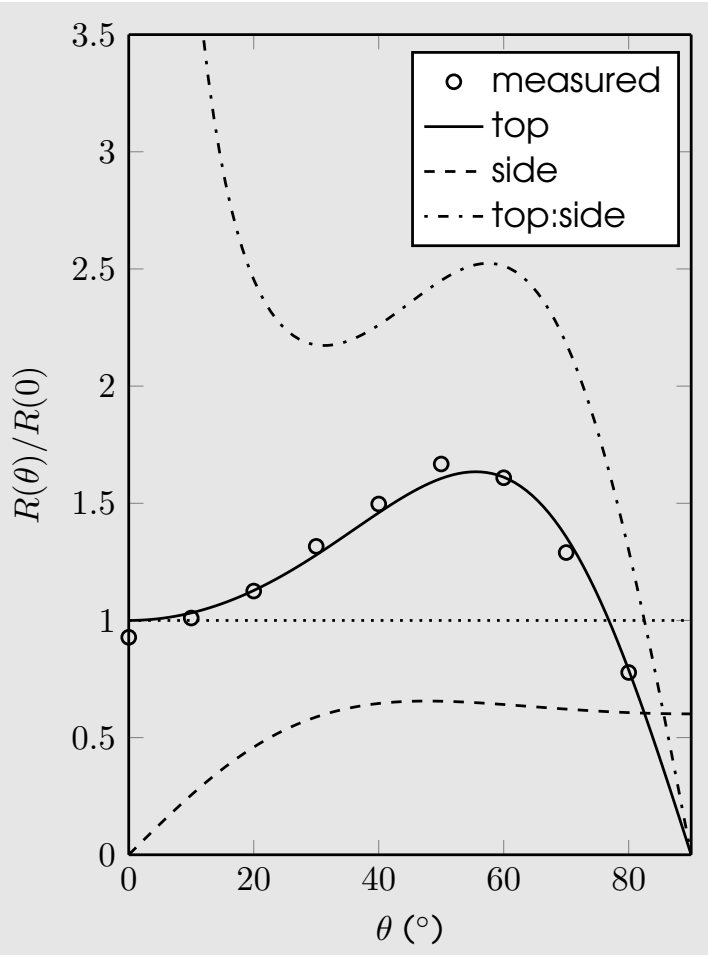

Figure 3.5: Plot of the relative etch rates for ion milling of silicon oxide as a function of angle of incidence for a horizontal surface ('top'), a vertical surface ('side') and the ratio of these two ('top:side'). The measurements to which the 'top' curve was fitted are also shown, normalized with the $R(0)$ found from the fitting.

etched, while the horizontal surfaces are etched normally, so the ratio approaches infinity as $\theta$ approaches 0 . This will allow selective etching of horizontal surfaces but because of the (almost) normal incidence there is hardly a shadowing effect anymore. At around $30^{\circ}$ the etching angle for vertical surfaces is close to the optimum etching angle for most of the time that it is exposed to the beam, while the etch rate for horizontal surfaces is still very close to that for normal incidence, and therefore the etch speed ratio reaches a minimum. At around $60^{\circ}$ the etch rate for horizontal surfaces is quite optimal, while for vertical 


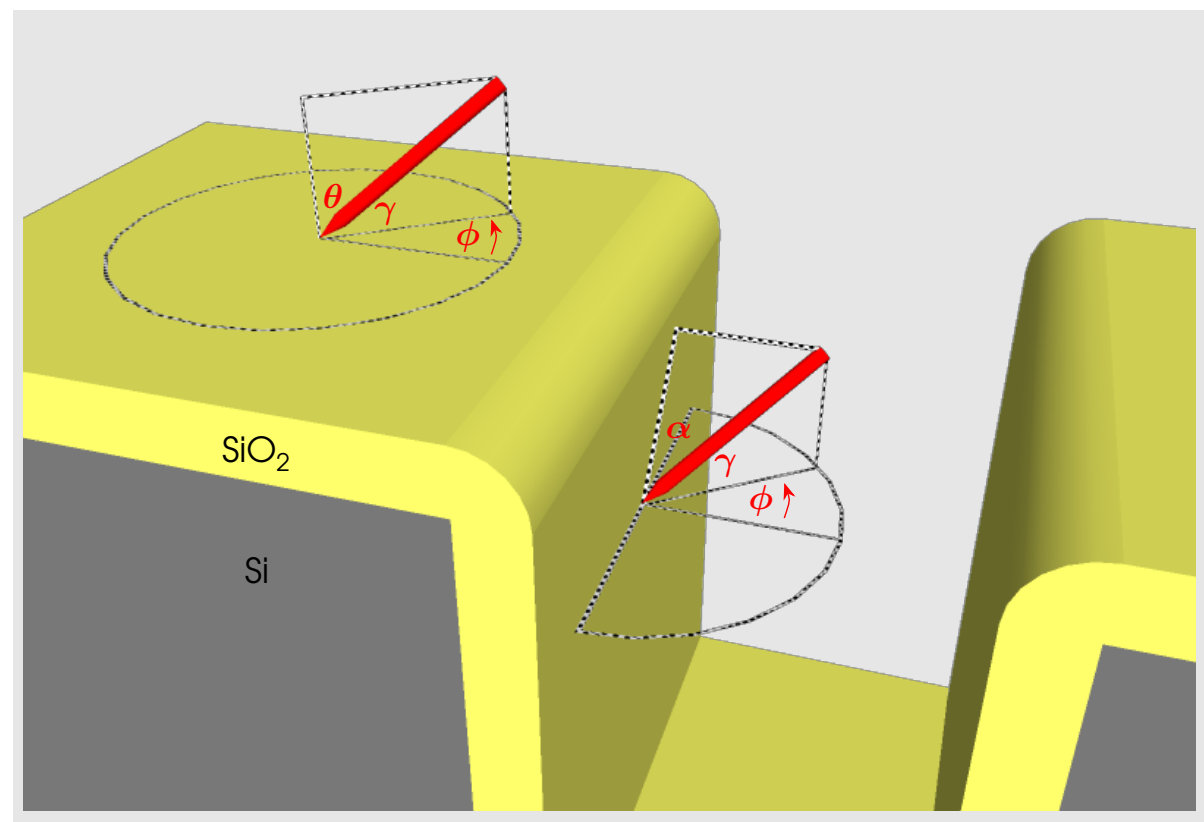

Figure 3.6: Sschematic representation of a rotating 3D silicon structure with a conformally grown layer of silicon oxide, which is etched at various angles by the ion beam (red 'pencils'), depending on the orientation.

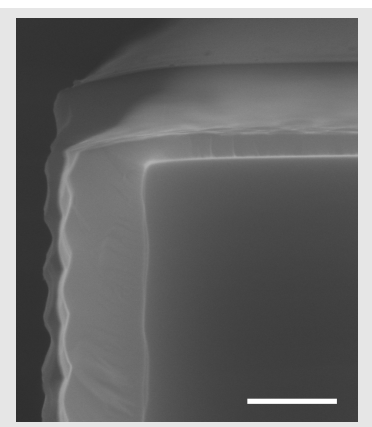

Figure 3.7: Overlay of a cross-sectional SEM images of an oxidized silicon structure that was subjected to ion beam etching under $57^{\circ}$ and one that was not etched. The scale bar is $500 \mathrm{~nm}$. 


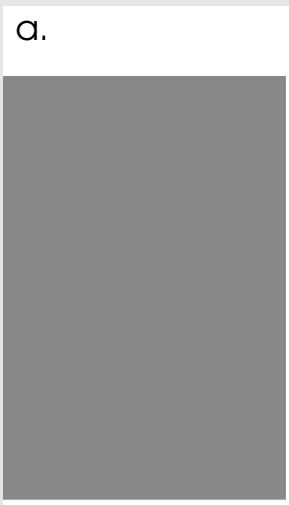

b.

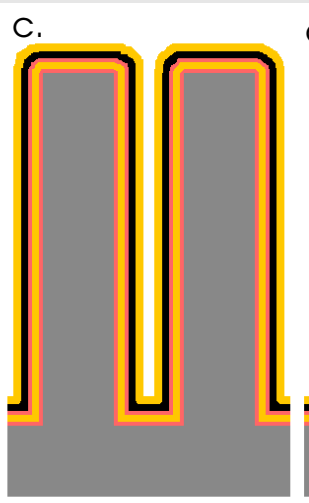

d.

e.

$f$.

g.

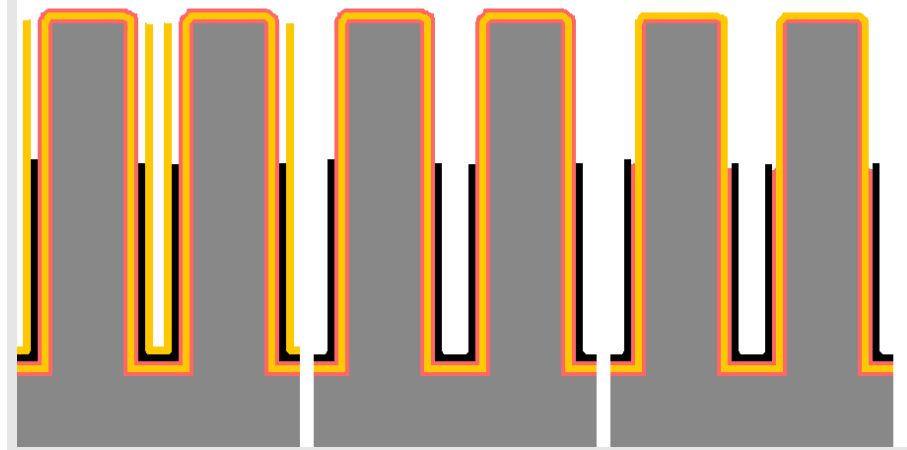

Figure 3.8: First part of the schematic process outline for opening only the bottom part of silicon structures ( 1 silicon substrate, silicon oxide,

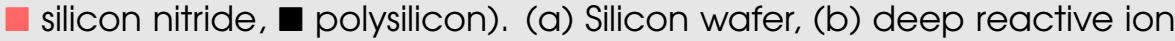
etching of patterned wafer to create vertical sidewalls, (c) conformal coverage of all structures with subsequently silicon nitride, silicon oxide, silicon nitride, polysilicon and silicon oxide, (d) removal of silicon oxide from the top, (e) isotropic etching of polysilicon to the desired height, $(f)$ isotropic etching of silicon oxide, $(g)$ isotropic etching of silicon nitride. Continued in Figure 3.9. 


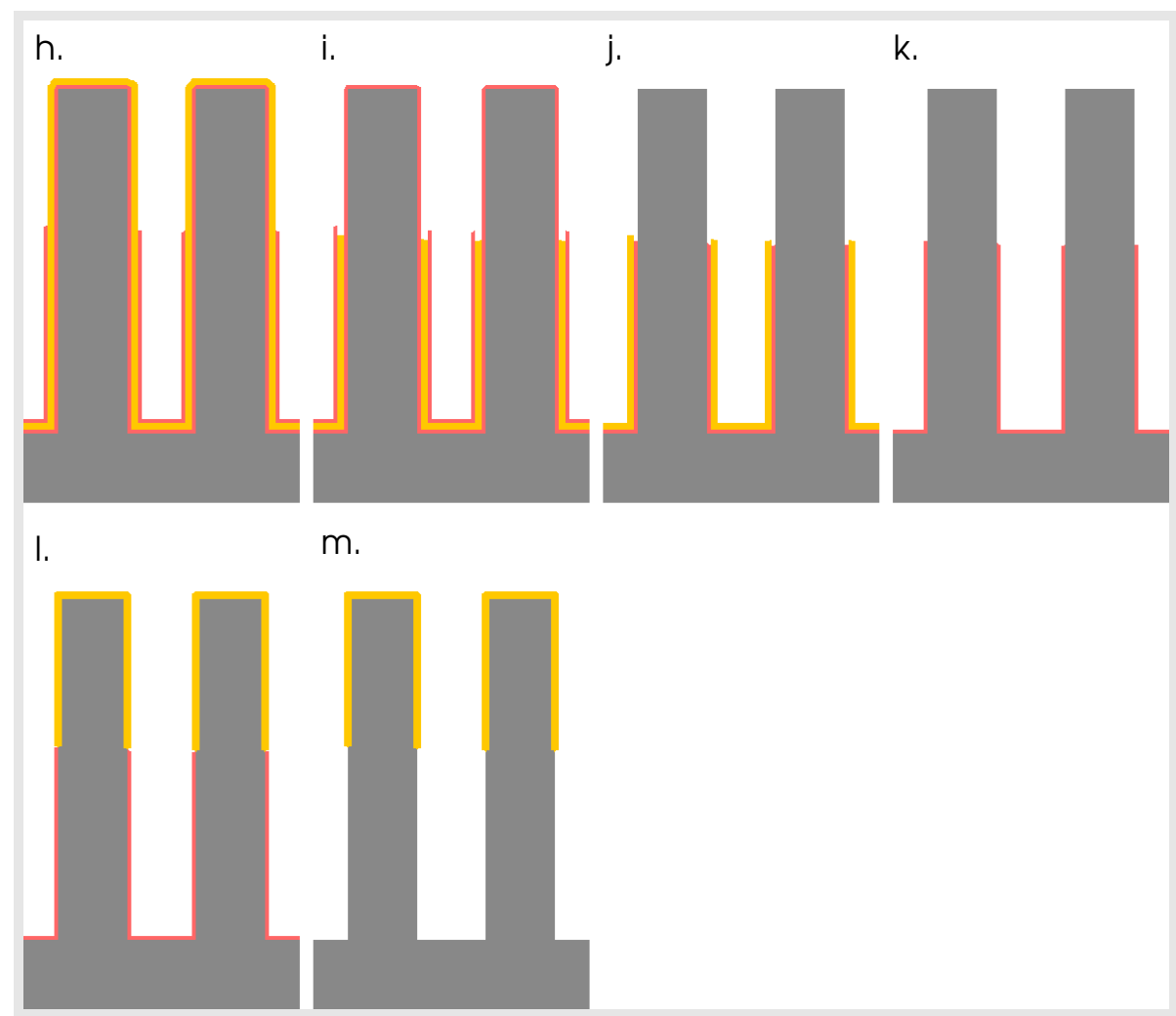

Figure 3.9: Schematic process outline for opening only the bottom part of silicon structures, continued from Figure 3.8 ( 1 silicon substrate, silicon oxide, silicon nitride, polysilicon). (h) Isotropic etching of polysilicon, (i) isotropic etching of silicon oxide (j) isotropic etching of silicon nitride, $(\mathrm{k})$ isotropic etching of silicon oxide, (I) local oxidation of silicon and $(m)$ isotropic etching of silicon nitride. See text for a more detailed description. 

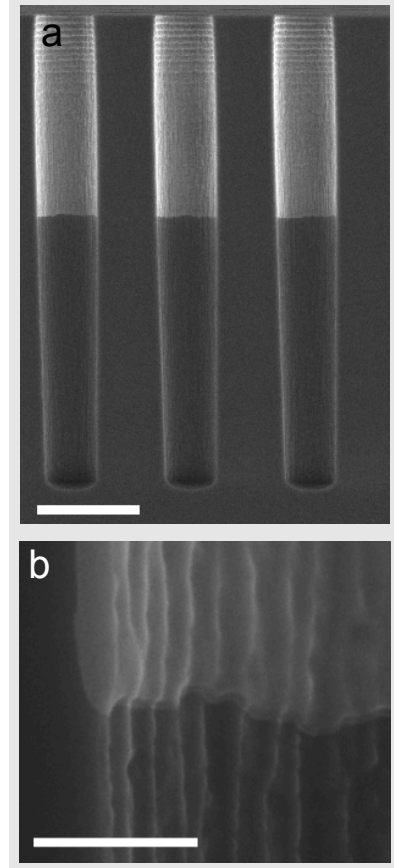
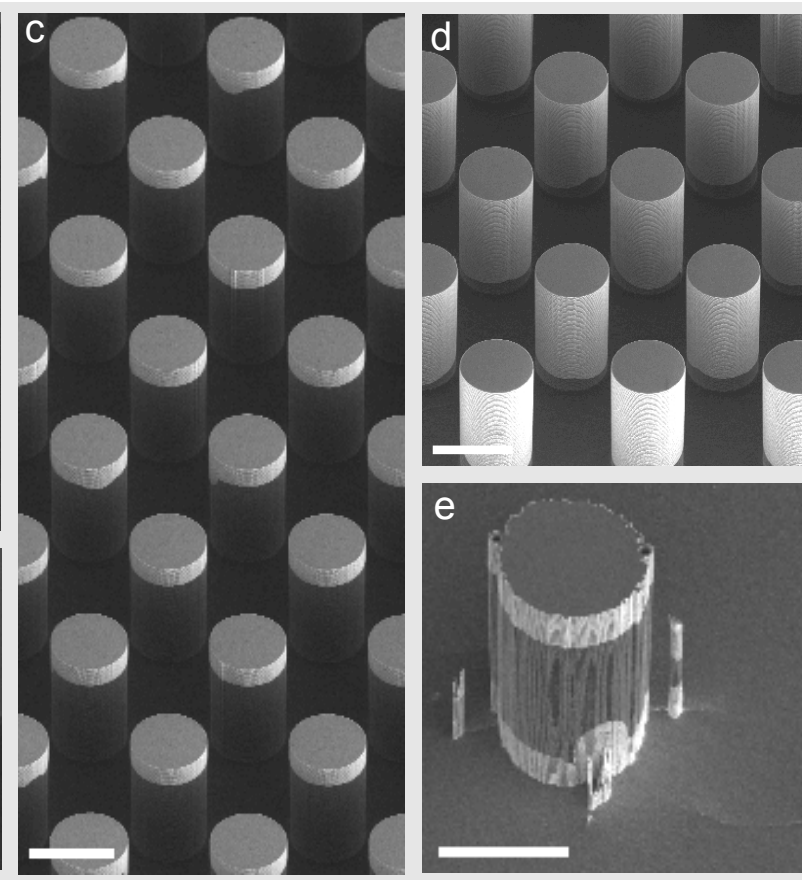

Figure 3.10: SEM images of silicon structures of which only the top part was covered with silicon oxide (lighter gray): (a) microholes, (b) close up of a sidewall at the point where the silicon oxide starts, micropillars with (c) a small and (d) a large part is covered by silicon oxide, and (d) a structure where the silicon (darker gray) is only exposed at the middle of the sidewalls, while everything else is covered in silicon oxide. Scale bars: (a) 5, (b) 0.5 , (c) 25 , (d) 25 and (e) $25 \mu \mathrm{m}$.

surfaces the average etch speed is similar for 30 and $60^{\circ}$, so here the ratio is reaches a maximum, and horizontal surfaces can be etched with some selectivity. Additionally, since the beam is now significantly tilted, clear shadows will be cast by the structures. Further increasing the angle has little effect on the etch rate of vertical surfaces, while the horizontal surfaces are hardly etched anymore because the beam becomes increasingly parallel to these surfaces. This would allow selective etching of vertical surfaces; however, for most patterns they would be in the shadow of another structure most of the time. 
For what seems to be the ideal angle for selective etching from Figure $3.5,57^{\circ}$, thermally oxidized silicon ridges formed by deep reactive ion etching were subjected to ion beam etching for $30 \mathrm{~min}$, broken, and then imaged by SEM. In this experiment we have chosen to not completely remove the oxide from the top, because in that case there would be no reference surface for determining the etch rate on the top (except when the etching process would have stopped exactly at the silicon oxide-silicon interface, which is difficult to achieve with ion beam etching). Also, ridges from the same wafer that were not etched by an ion beam were broken and imaged.

Figure 3.7 shows an overlay of a thermally oxidized $\left(90 \mathrm{~min}, 1000^{\circ} \mathrm{C}\right.$, water vapor atmosphere) structure that was subjected to ion beam etching at $57^{\circ}$, and one that was not, both from the same wafer. From the top, on average about $392 \mathrm{~nm}$ is removed, while from the sidewall on average only $117 \mathrm{~nm}$ was removed. This means the etching ratio between horizontal and vertical surfaces is in this case 3.35, which is higher than the factor of about 2.5 that would be expected from Figure 3.5. That means that the technique works even better than expected. This improvement may be caused by the roughness of the 'vertical' silicon oxide surfaces resulting from the Bosch etching recipe that was used to shape the original silicon structure. Because of this roughness there is a local variation in sidewall angle, resulting in an etch speed lower than predicted by the model, which assumed a smooth vertical surface.

The minimum aspect ratio for which the shadowing effect works is determined by the chosen ion beam angle. For an angle $\theta$, a structure with height $h$ will cast shadows up to $h \sin \theta$ away, so $h \cdot 0.84$ for $\theta=57^{\circ}$. Every lower horizontal surface that is further away from a structure than this distance will be etched with the same speed as the top surfaces. However since some overetch is always needed to be sure of complete silicon oxide removal at all top surfaces, this aspect ratio requirement will in practice be even more stringent, since the bottom surfaces are not always in the shadow, so even the parts of these surfaces that are within this distance have a finite etch speed.

The consequences of this aspect ratio limitation were seen in Figure 3.3(c). Most parts of the large lower-lying horizontal surfaces that surround these structures were never in the shadow of any structure, and therefore in step $d$ as shown in Figure 3.2, the silicon oxide on these surfaces etches with the same speed as the horizontal silicon oxide surfaces on top of the silicon structures. Therefore the silicon oxide is also 
completely removed from the large lower-lying surfaces in this step. The subsequent retraction of the polysilicon layer underneath the silicon oxide layer will then proceed along the vertical walls from both the top and the bottom, leaving only a band of silicon oxide halfway along the vertical surfaces in the final device, which is shown clearly in Figure 3.3(c).

\subsection{Modify the bottom part of a structure}

This section describes the technique by which the bottom part of a silicon micro-/nanostructure can be exposed selectively, while keeping the top part covered in silicon oxide, as shown in Figure 3.1b.

\subsubsection{Fabrication}

The fabrication procedure is shown schematically in Figures 3.8 and 3.9. Steps (a-j) are the same as for the process described above for only covering the top part of a structure, except that in step (c), before all the other layers, another $50 \mathrm{~nm}$ layer of silicon rich nitride is deposited by LPCVD as described in Section 3.2.2 for step (c) of that process. Also, in step (j) the hot phosphoric acid solution etches both layers of silicon rich nitride: the outer layer is completely removed, and the inner layer is patterned by the silicon oxide hard mask that remains.

The extra silicon nitride layer and the LOCOS step are necessary to invert the pattern that was created in the same way as in the procedure for opening the top part of a structure. Such an inversion requires the transformation of a thin layer of one material into another in a controlled way. This additional requirement further limits the choices of materials and methods with which this technique could be achieved.

After step $(j)$ the procedure for opening the bottom part of the silicon structure continues as follows: (k) the silicon oxide is removed by etching $2 \mathrm{~min}$ in BHF as in step (i), and (I) on the top part $100 \mathrm{~nm}$ of silicon oxide is formed by dry thermal oxidation while the bottom part is prevented from oxidation by the silicon rich nitride layer (LOCOS, $11 \mathrm{~h}$, $900^{\circ} \mathrm{C}$ ). (m) Finally the bottom part of the structure is uncovered by 


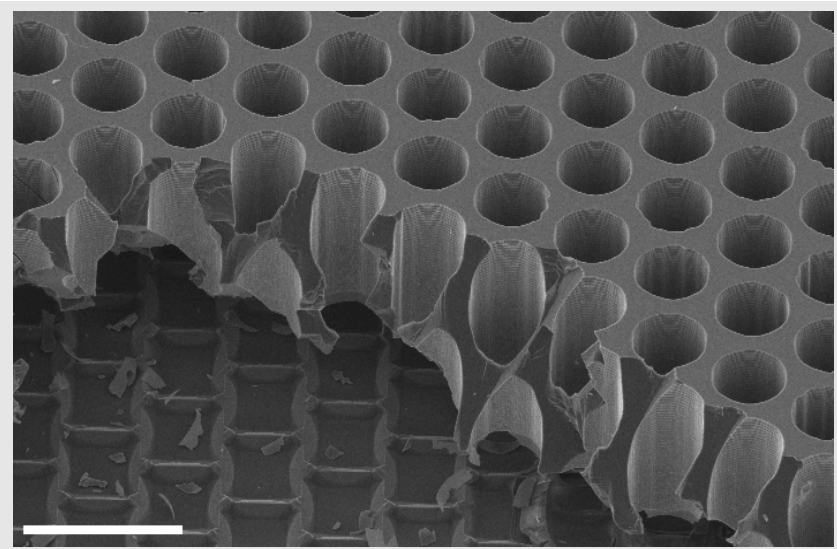

Figure 3.11: SEM image of a free-hanging silicon membrane created by underetching structures of which only the top was covered by silicon oxide (lighter gray). The scale bar is $25 \mu \mathrm{m}$.

etching the silicon rich nitride in hot phosphoric acid for 25 min as in $\operatorname{step}(\mathrm{g})$.

A properly patterned silicon nitride layer is already formed in step (g). However the polysilicon layer could not be removed without damaging the silicon structures if the underlying layers and subsequent steps were not used.

To give an example of the applicability of this process, after the final processing step an additional silicon etching step was performed to obtain the free standing membrane shown in Figure 3.11. After step $(\mathrm{m})$ the sample was treated in $1 \%$ aqueous HF solution for $1 \mathrm{~min}$ and subsequently etched in TMAH solution as described above for $10 \mathrm{~min}$.

\subsubsection{Results and discussion}

Figure 3.10 shows some examples of the structures obtained by applying the bottom modification process to silicon structures. Again the procedure can be applied to both holes (Figure 3.10(a)) and pillars (Figure 3.10(c) and (d)) and the transition can be controlled to be at various heights (Figure 3.10(c) and (d)) and is well defined (Figure 3.10(b)). The only differences with the top modification process 

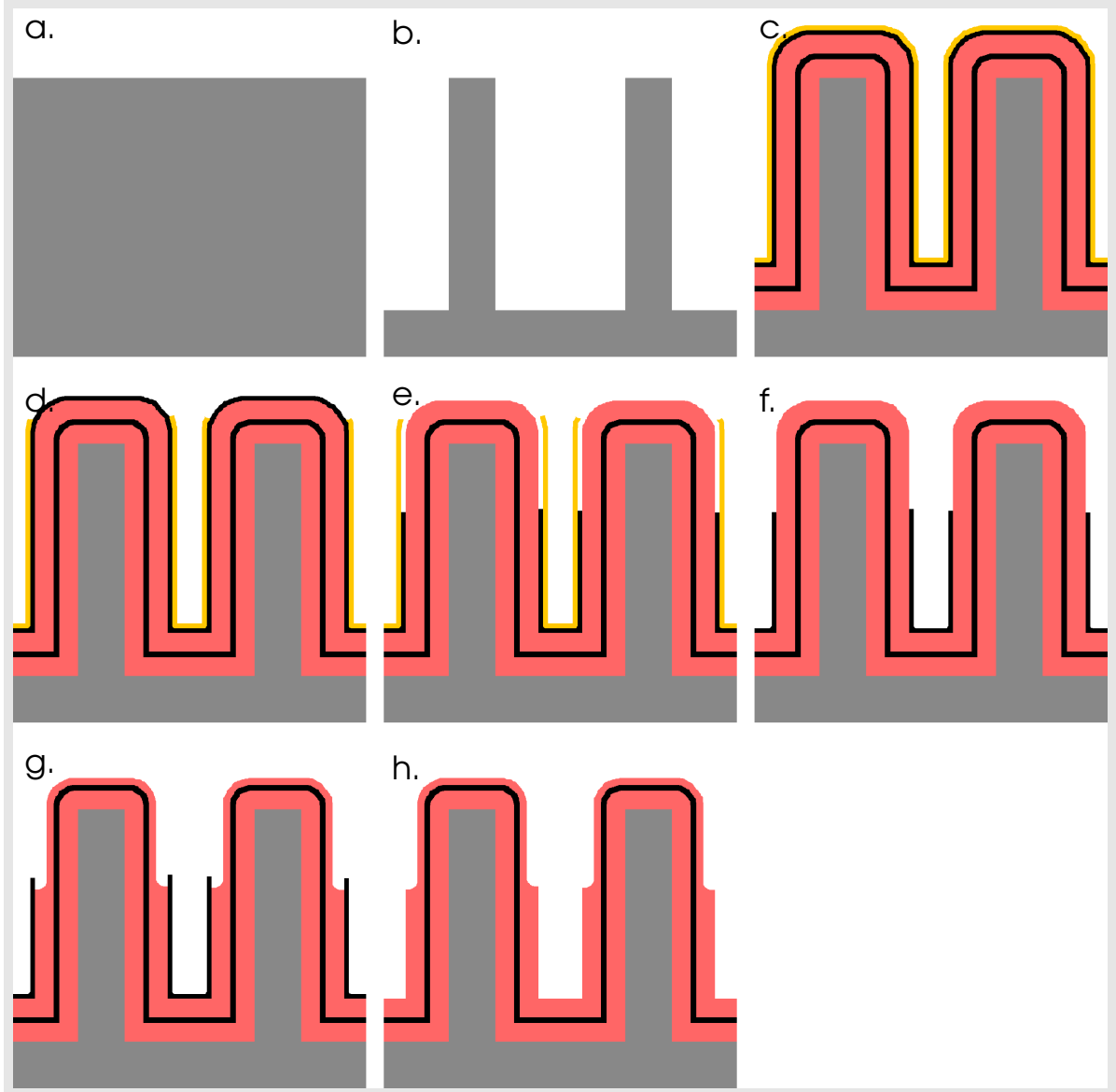

Figure 3.12: First part of the schematic process outline for opening only a horizontal band on silicon structures ( silicon substrate, " silicon oxide, silicon nitride, $\square$ polysilicon). (a) Silicon wafer, (b) deep reactive ion etching of patterned wafer to create vertical sidewalls, (c) conformal coverage of all structures with subsequently silicon nitride, polysilicon, silicon nitride, polysilicon and silicon oxide, (d) removal of silicon oxide from the top, (e) isotropic etching of polysilicon to the desired height, $(f)$ isotropic etching of silicon oxide, $(g)$ timed isotropic etching of silicon nitride, $(h)$ isotropic etching of polysilicon. Continued in Figure 3.13. 


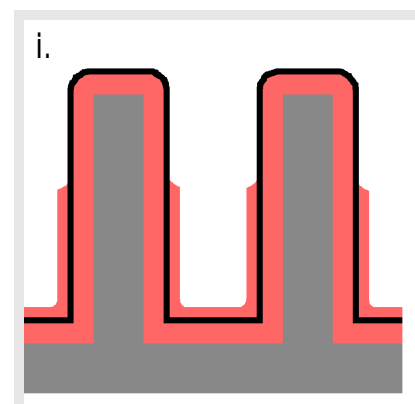

I.

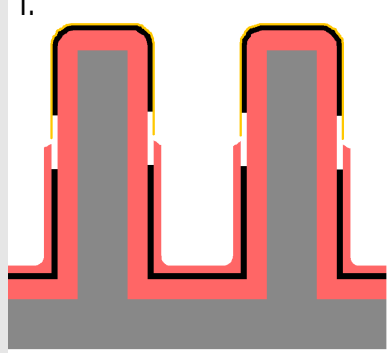

O.

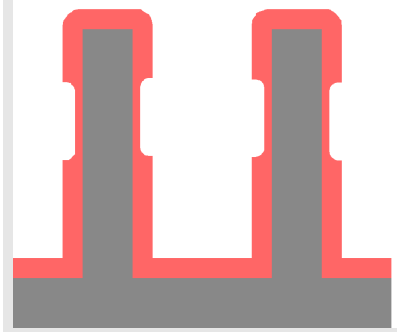

j.

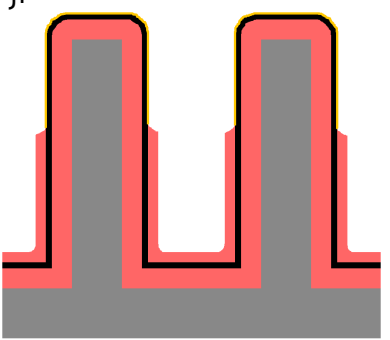

m.
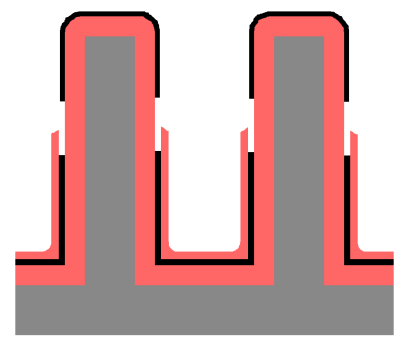

p. k.

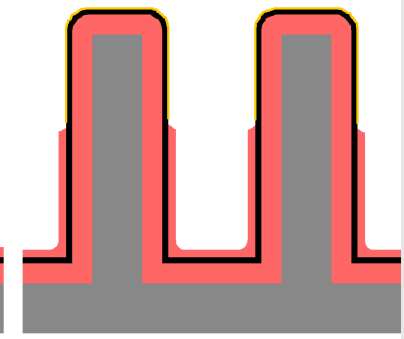

n.

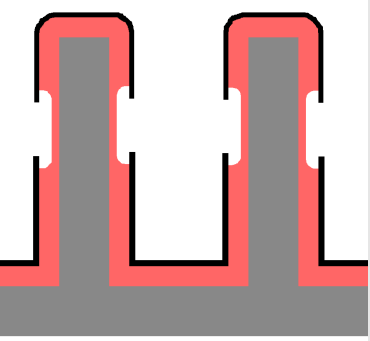

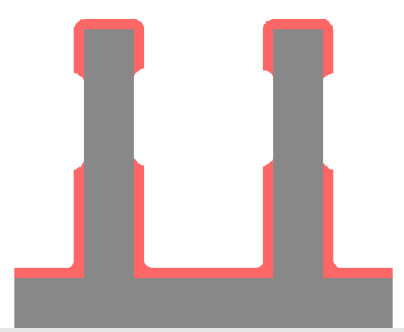

Figure 3.13: Schematic process outline for opening only a horizontal band on silicon structures, continued from Figure 3.12 ( silicon substrate, silicon oxide, silicon nitride, $\mathbf{a}$ polysilicon). (i) (See Figure 3.14 for a close up view of steps $i-1$ ) isotropic etching of silicon nitride (j) local oxidation of the polysilicon (not the full thickness), (k) timed isotropic etching of less than the full thickness of the silicon nitride, (I) isotropic etching of polysilicon to the desired band width $(m)$ isotropic etching of silicon oxide $(n)$ timed isotropic etching of silicon nitride (o) isotropic etching of polysilicon $(p)$ timed isotropic etching of silicon nitride. See text for a more detailed description. 
is that the areas covered by silicon oxide and the uncovered areas are now interchanged. This is also true for the band patterns that are formed on structures surrounded by large open areas, as seen in Figure 3.10(e), which also shows again the roughness tolerance of this technique.

An example of a structure resulting from another silicon etching step after the final process step is shown in Figure 3.11. The silicon was completely removed from the lower part of the structure, leaving only the top part: a free standing perforated silicon membrane.

\subsection{Modification at an intermediate height}

This section describes the technique by which the middle part of a silicon micro/nanostructure can be exposed selectively, while keeping the bottom and top parts covered in silicon nitride, as shown in Figure 3.1c.

\subsubsection{Fabrication}

The fabrication procedure is shown schematically in Figures 3.12 and 3.13, and details are given below.

In principle, the same ideas are used as for the first two processes, but for the definition of the band another newly developed procedure was added here. The transition point between the top and bottom parts of the structure that was defined in the other processes is used here as a starting point to define a band locally. This procedure is shown in more detail in Figure 3.14. The LOCOS is used to grow a silicon oxide that starts exactly where the silicon nitride stops. Subsequent isotropic thinning of the silicon nitride layer widens this transition point so that a narrow line of the layer underneath (polycrystalline silicon) can be accessed, and isotropically etched/retracted to define the desired band. This technique is comparable to the process reported previously by Berenschot et al. ${ }^{66}$, where a larger feature was developed by etching a polysilicon layer through a nanoscale opening.

Another difference is that no oxide layer is used to facilitate the removal of the two polysilicon hard mask layers. Instead, the polysilicon 


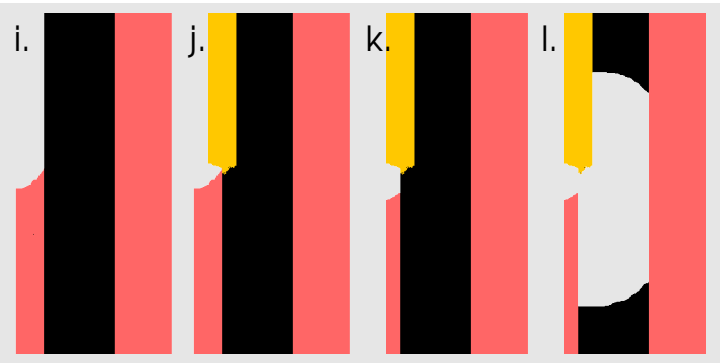

Figure 3.14: Close-up view of part of the process shown in Figure 3.13 ( silicon oxide, silicon nitride, $\mathbf{\square}$ polysilicon). (i) After stripping silicon oxide, a silicon nitride hard mask is left on the polysilicon (j) local oxidation of the polysilicon (not the full thickness), (k) isotropic etching of half the thickness of the silicon nitride, (I) isotropic etching of polysilicon.

hard mask is only used for partially etching the silicon nitride layer underneath. Because the silicon nitride was not etched completely, the polysilicon hard mask can then be removed without damaging anything underneath the silicon nitride layer. After removal of the polysilicon, the patterning of the silicon nitride is completed by etching until the previously thinned region has completely been etched away, leaving a layer of silicon nitride only in the regions which were previously covered by the polysilicon hard mask.

\subsubsection{Fabrication details}

This section gives a detailed description of the steps that are summarized in Figures 3.12 and 3.13.

The same process steps are used as for the other processes, only in a different order, so they are described more briefly over here, except where more details are needed. (a) The process is again done on a silicon wafer into which vertical structures are etched (b) using a silicon oxide hard mask, followed by cleaning and stripping of the mask and fluorocarbons. (c) Conformal layers are formed subsequently by LPCVD: $400 \mathrm{~nm}$ of silicon rich nitride (100 min deposition), $100 \mathrm{~nm}$ of polysilicon (28 min), $400 \mathrm{~nm}$ of silicon rich nitride and $170 \mathrm{~nm}$ of polysilicon. The outer $70 \mathrm{~nm}$ or so of polysilicon is again oxidized to form $135 \mathrm{~nm}$ of thermal oxide as an outer layer. 
Steps (d) through (h) are the same as in the previous processes, except for some timing variations. In step (d) the etching was done for 20 , 40 or $60 \mathrm{~min}$ on different wafers, and in step (g) the etching of the silicon nitride was timed carefully $(60 \mathrm{~min})$ such that only $250 \mathrm{~nm}$ of the nitride is etched, and some of it remains. Subsequently (i) the silicon nitride is etched further (hot phosphoric acid, $50 \mathrm{~min}$ ) such that the underlying polysilicon is exposed only where the silicon nitride was already made thinner before.

A close up view of steps (i) to (I) is shown in Figure 3.14. (j) The top part of the structure is covered again with $50 \mathrm{~nm}$ of silicon oxide by thermal oxidation ( $2 \mathrm{~h}$ and $43 \mathrm{~min}, 900^{\circ} \mathrm{C}$, dry oxidation) of the outer $25 \mathrm{~nm}$ of the exposed polysilicon (LOCOS process). (k) Isotropic etching of silicon rich nitride in hot phosphoric acid for 12 min results in thinning of the layer, but also in retraction of the layer at its edges, thereby exposing a very narrow line of polysilicon exactly where the LOCOS interface was positioned. From this starting point the polysilicon can now be retracted below the combined hard mask of silicon rich nitride and silicon oxide by etching isotropically in TMAH (preceded by $1 \mathrm{~min}$ etching in $1 \% \mathrm{HF}$ and rinsing), and the etching time controls the width of the band created. (Here we etched for 1, 36 and 6 minutes to show different band widths) The hard mask materials are then removed by $(m)$ etching the silicon oxide for 1 min in BHF and (n) etching the silicon rich nitride in hot phosphoric acid for $60 \mathrm{~min}$.

The latter also transfers the pattern in the polysilicon layer to the silicon rich nitride layer underneath (but like before, it only creates a thinner region, the layer is not fully etched through). Step (o) and (p) are now the same as steps $(h)$ and (i), and result in a silicon nitride layer everywhere except for a band 'halfway' the silicon structure.

For the formation of doped regions halfway the pillar, in step (c) in Figure 3.12 a layer of silicon oxide was formed on the silicon by thermal oxidation, before the deposition of the other layers. After step $(p)$, this silicon oxide layer was patterned by BHF etching. Subsequently, $300 \mathrm{~nm}$ of phosphosilicate glass was deposited conformally on the structures by LPCVD ( $350 \mathrm{mTorr}, 675^{\circ} \mathrm{C}, 30 \mathrm{SCCM} \mathrm{N}$ through the tetraethyl orthosilicate (TEOS) evaporator to form a $50 \mathrm{SCCM}$ TEOS/ $\mathrm{N}_{2}$ mixture, $150 \mathrm{SCCM}$ $\mathrm{O}_{2}, 16.5 \mathrm{SCCM} \mathrm{PH}$ and $313.5 \mathrm{SCCM}$ argon). The phosphorous was made to diffuse into the silicon by heating the samples at $1050{ }^{\circ} \mathrm{C}$ for $15 \mathrm{~min}$ in nitrogen atmosphere, which should lead to a surface doping concentration of above $1 \times 10^{19} \mathrm{~cm}^{-3}$, gradually decreasing to the background 

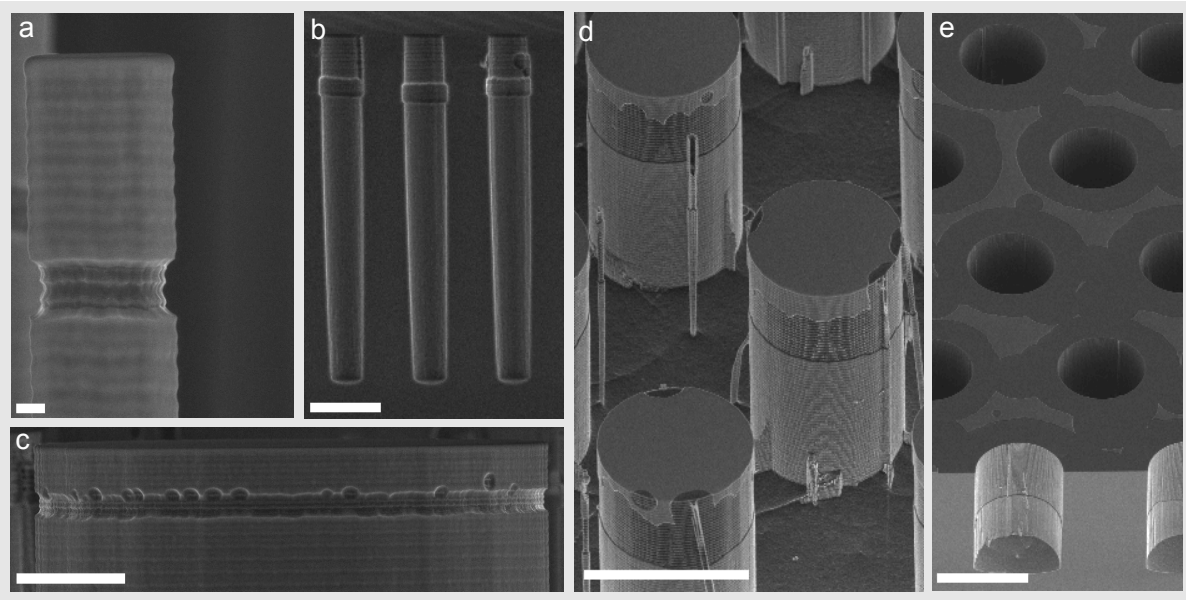

Figure 3. 15: SEM images of various silicon microstructures from which a narrow band of silicon (darker gray) was uncovered, while the remainder of the structure remained covered in silicon nitride: (a) narrow micropillars, (b) narrow microholes and (c) wide micropillars, all with 20 and $1 \mathrm{~min}$ polysilicon retraction for the height and band with definition respectively, and further (d) wide micropillars where 40 and $36 \mathrm{~min}$ of retraction was used respectively, and (e) wide microholes where 60 and 6 min of retraction was used. Scale bars: (a) 0.5 , (b) 5 , (c) 5 , (d) 25 and (e) $25 \mu \mathrm{m}$.

boron doping level of around $1 \times 10^{15} \mathrm{~cm}^{-3}$ within $1 \mu \mathrm{m}^{6}$. Subsequently the phosphosilicate glass was stripped in BHF, as described above for silicon oxide etching, for $90 \mathrm{~s}$. Vertical cross sections were made using focused ion beam etching, and staining of the doped areas was done as we did before ${ }^{6}$.

\subsubsection{Results and discussion}

Figure 3.15(a) to (c) shows structures where the polysilicon retraction shown in step e in Figure 3.12 was performed for $20 \mathrm{~min}$ and in step I for $1 \mathrm{~min}$. Indeed a narrow band of silicon nitride was removed near the top of narrow (Figure 3.15(a)) and wide (Figure 3.15(c)) micropillars and for microholes (Figure 3.15(b)). However, some structures show holes in 
the silicon nitride on the sidewalls above the band (Figures 3.15(b) and (c)). This effect is even more pronounced when the polysilicon retraction times are increased (Figure 3.15(d)) and for some structures the silicon nitride is completely removed from the upper part of the sidewalls (Figure 3.15(e)). This is probably caused by a failure of the silicon oxide layer formed in step $\mathrm{j}$ as shown in Figure 3.13. The rough sidewalls of the structures, resulting from the Bosch etching process by which they were formed, causes strong local curvatures in the conformal layers that are formed on these sidewalls. This local curvature causes stress during the oxidation step, resulting in locally thinner silicon oxide points, especially since low temperature $\left(900^{\circ} \mathrm{C}\right)$ dry oxidation was used. The hot phosphoric acid and TMAH that are used in the subsequent steps also have a finite etch speed for silicon oxide, such that pinholes in the silicon oxide layer are formed at these thinner points. The polysilicon retraction step now also retracts the polysilicon with such pinholes as starting points, causing circular defects in the polysilicon, or even complete removal of the polysilicon in these regions for longer etching times.

However, this problem can probably be resolved by growing a thicker silicon oxide. It might also help to reduce the thinning time of the silicon nitride in step k as shown in Figure 3.13, since especially the selectivity of the hot phosphoric acid etchant between silicon nitride and silicon oxide is poor (roughly $10: 1$ ).

Despite some remaining engineering challenges, the feasibility of this procedure for creating an open band on a sidewall was clearly shown by the results for short TMAH etching times. Again in this case well-defined transitions were defined for both microholes and micropillars of different sizes.

To show that the band pattern can also be used for functional local modification along the sidewalls of silicon structures, the remaining dielectric pattern was used as a diffusion barrier during a diffusion doping process. A pillar was then cut in half along its axis using focused ion beam milling, and the doped regions were etched using a staining solution. Figure 3.16 shows the silicon micropillars with the opened bands. Visualization of the doping inside the pillar is shown in Figure 3.17. Although the contrast is weak, a lighter area can be seen on both sides of the pillar, which is the doped region, that was etched faster by the staining solution than the undoped silicon. 


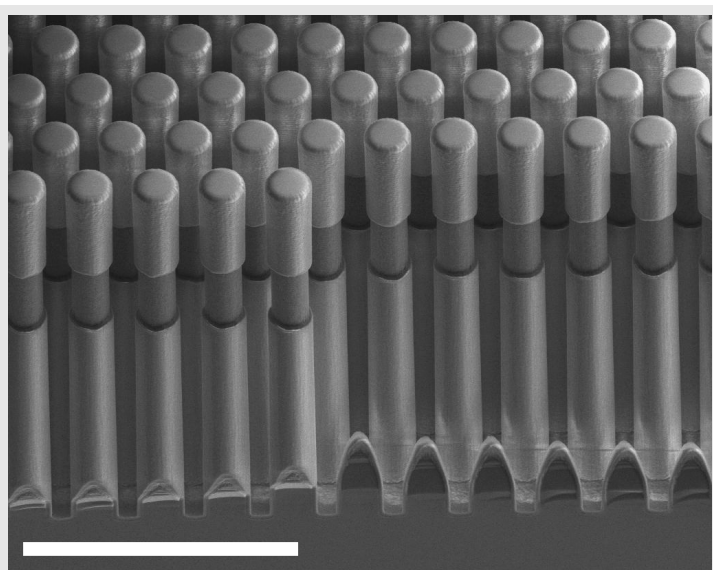

Figure 3.16: SEM image of silicon pillars covered by dielectric layers from which a band has been removed. The scale bar is $25 \mu \mathrm{m}$.

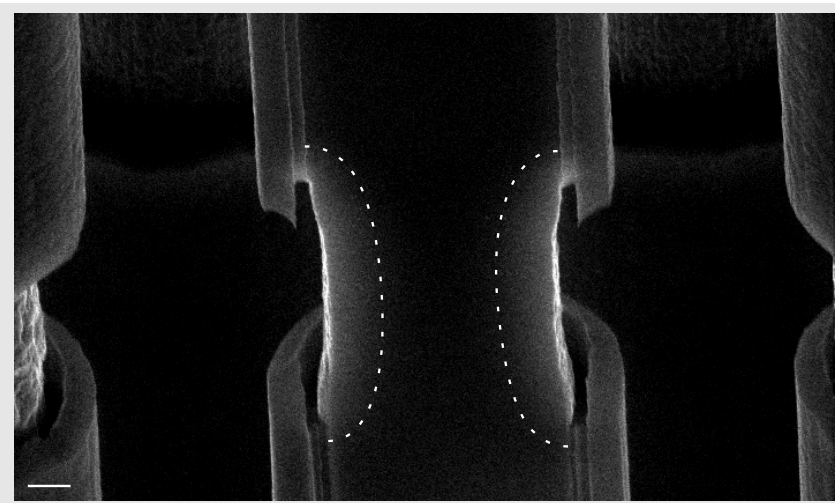

Figure 3.17: SEM image of silicon pillars on which a dielectric diffusion barrier has been used during the doping process, highlighted by the dotted line. In this way, a confined doped region is obtained at a defined position along the pillar length. This has been visualized by FIB cross sectioning and chemical staining. The scale bar is $500 \mathrm{~nm}$. 


\subsection{Conclusion}

Versatile, integrated circuit technology compatible wafer-scale processes for patterning sidewalls of silicon structures were presented and successfully demonstrated in this study. The impact angle dependent etch rate of silicon oxide in an ion beam etching system was characterized, and it was shown that this angle dependence can be used for opening horizontal silicon oxide surfaces while leaving vertical surfaces protected. The retraction of polysilicon underneath a dielectric layer was shown to be a reliable, well-controlled and roughness insensitive method for defining a hard mask edge with an offset to an existing edge, without additional optical lithography steps. A smart combination of these techniques can be used to selectively open any desired region along the vertical sidewall of a structure, as long as the open space between such structures is smaller than the height of the structures.

The resolution of these techniques is in the order of magnitude of the layer thicknesses used. Therefore it can, in principle, be scaled down to the nanometer range by using thinner layers. However, at the layer thicknesses used in the present study (50 to $400 \mathrm{~nm}$ ) defects have been detected for certain geometries. Therefore, the downscaling of especially the silicon oxide layer thicknesses will need to be accompanied by careful tuning of etching parameters in several of the fabrication steps involved. Furthermore, the retraction of a thinner polysilicon layer by wet chemical etching may lose the linear dependence on etch time as the etch rate may become transport limited.

It has been shown that the locally opened regions can be used for selective deposition, modification or removal of material at an arbitrary height along the vertical sidewall of silicon structures. One very relevant recent application in which we have used part of the methods described here is in the local coverage of silicon pillars with an electrocatalyst for the purpose of solar hydrogen generation, as described in Chapter 5. The demonstrated applications are only examples, and these techniques can be considered for application in many other fields. From the references cited in Section 3.1, it can be seen that 3D micro and nanostructures are used in a broad range of fields. Some examples are micro and nanoelectronics, scanning probe microscopy, (bio)sensors, MEMS/NEMS, microfluidics, nanostructured surfaces, cell studies, catalysis, photonics, energy conversion and stor- 
age, supercapacitors, tissue engineering, filtration, chromatography, healthcare, photovoltaics, computer memory, mixing, acoustics and thermoelectrics. The demonstrated fabrication methods are potentially useful in any of these fields if the parallel fabrication of large amounts of 3D micro and nanostructures is desirable. 


\section{Chapter 4}

\section{Microfabrication techniques for repeating structures in the out-of-plane direction}

This Chapter presents a microfabrication technique that was developed to allow multiple modifications in every silicon micropillar in an array. It is the next step after the technique that was described in Chapter 3: like that technique here more control is gained in the out-ofplane direction with using only wafer-scale technology. However while a contrast could only be defined in one or two locations in the previous Chapter, here a technique is presented that allows the definition of many of these lines above each other. It makes use of a specially developed variation of Bosch etching, combined with corner lithography, and in one case also with digital etching and edge lithography. The technique shows promise for the fabrication of large arrays of structures with features repeated in the vertical direction, which could help to make better use of the third dimension in a variety of microfabricated devices. 
a.

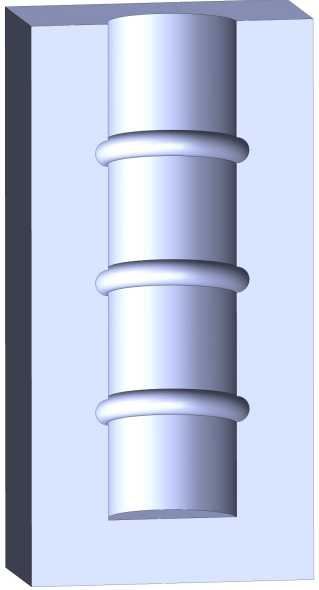

b.

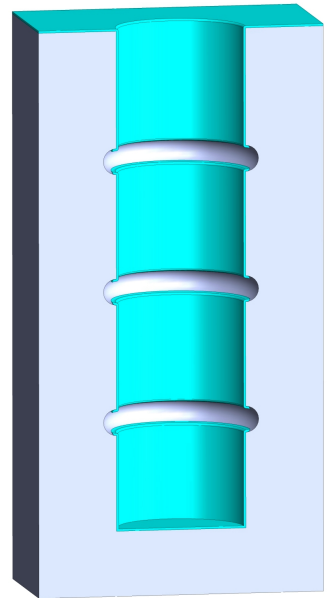

C.

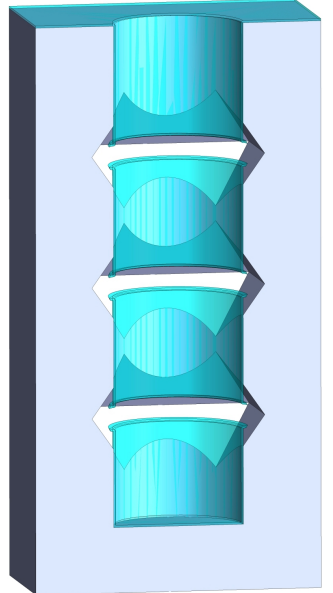

Figure 4.1: Visual summary of the technique described in this Chapter. (a) Illustration of a structure with multiple big scallops at different heights along the sidewall; (b) illustration of the vertical mask resulting from corner lithography of the structure with multiple big scallops; blue represents silicon oxide and gray represents silicon; (c) example of a repeated structure that can be made with this mask with timed anisotropic etching of the bulk silicon ${ }^{67}$.

\subsection{Introduction}

Cerofolini et al. ${ }^{84}, 85$ reported the realization of repeated structures in the out-of-plane direction. However this technique requires the deposition of as many alternating layers as the number of repetitions that are required. The etching of vertical holes through these layer stacks is challenging because of the inconsistent etching behavior of the different materials. Moreover, the height of the resulting structure is limited by the deposition rate of the layers, and a maximum structure height of a few micrometers was achieved. In this Chapter, deep reactive ion etching (DRIE) and corner lithography are combined to create an outof-plane mask, which can be used for the fabrication of 3D structures that are repeated at defined depths in the vertical direction. 
Corner lithography ${ }^{60}$ is based on the filling of concave corners by a conformally deposited layer and the subsequent timed isotropic etching of the layer to leave a well-defined residue in the corner while on flat surfaces and convex corners the layer is already removed. The relation between the residue size, thickness of the deposited layer, and the isotropic etching depth has been summarized by Burouni et al. ${ }^{86}$. Using this residue, various structures can be fabricated. Berenschot et al. ${ }^{67}$ made use of a nitride residue as an inversion mask to fabricate octahedral and fractal structures. Yu et al. ${ }^{87}$ applied corner lithography to a 3D colloidal template to obtain nano-ring crystals. The structures resulting from corner lithography have been applied for example in specialized AFM probes ${ }^{88}$ and devices for cell trapping ${ }^{66}$, brain-on-achip ${ }^{89}$ and gas permeation ${ }^{90}$.

The Bosch process is one of the most commonly used methods to create silicon structures with high aspect ratios. This technique relies on repeated switching between isotropic etching and passivation to obtain a cavity with straight sidewalls ${ }^{91}$. By extending one of the etching steps in the sequence, Hirose et al. ${ }^{37}$ introduced a big scallop after a sequence of standard Bosch etching steps. Because of the shadowing effect of this structure the subsequently evaporated metal layer could be interrupted at this big scallop to avoid an electrical short cut. Marty et al. ${ }^{92}$ and Chang et al. ${ }^{93}$ combined the standard Bosch processing with a purely isotropic etching process to create multiple big scallops along sidewalls and indicated its potential application in photonics, electronics and microfluidics. In these examples the horizontal depth of the reported big scallops, and therefore their usefulness for corner lithography, was limited.

Here the big scallops are introduced with the use of a bottom passivation technique, which results in nearly perfect semi-circular big scallops at various depths, as illustrated in Figure 4.1(a). For forming a residue only inside these big scallops, two types of corner lithography were investigated: silicon nitride-based corner lithography, and a combination of digital etching-based ${ }^{94}$ corner lithography with edge lithography. As an illustration of the potential of this technique, the mask formed by these residues was inverted, as illustrated in Figure 4. 1(b), and features are etched at each big scallop (for example octahedra, as shown in Figure 4.1(c)). 


\subsection{Materials and Methods}

\subsubsection{Patterning of the mask}

Experiments were performed on silicon (100) wafers, which were first oxidized at $1150^{\circ} \mathrm{C}$ (wet oxidation) to form a $1 \mu \mathrm{m}$ thick oxide hard mask, and then patterned with $20 \mu \mathrm{m}$ diameter circles with $60 \mu \mathrm{m}$ edge-toedge separation via optical lithography and deep reactive ion etching of the exposed oxide (Adixen DE, $15 \mathrm{SCCM} \mathrm{CHF}_{3}, 20 \mathrm{SCCM} \mathrm{C} \mathrm{C}_{4} \mathrm{~F}_{8}$, 150 SCCM He, $8.5 \times 10^{-13}$ mbar, 280 W ICP, 350 W CCP, 150 s).

\subsubsection{DRIE: introduction of the big scallops along the side- wall.}

A standard Bosch process was applied, which started with deposition and ended with etching, with the result that no fluorocarbon was deposited on the last scallop at the end of the process, as shown in Figure 4.2(b). The recipe for the standard Bosch process is shown in Table 4.1 and was performed in an SPTS Pegasus plasma etching machine. Subsequently, the newly developed bottom passivation technique was applied, which started with $2 \mathrm{~s}$ of directional plasma oxidation to selectively passivate the bottom surface (SPTS Pegasus, 200 SCCM O 2,5 mTorr, 2500 W ICP, 100 W CCP, $20 \%$ LF pulse), as shown in Figure 4.2(c). Isotropic, radical-dominated etching (no ion acceleration power) was then used to develop a highly isotropic big scallop from the sidewall of the last small scallop, as illustrated in Figure 4.2(d) (SPTS Pegasus, 275 SCCM SF $_{6}, 26$ mTorr, $2200 \mathrm{~W}$ ICP, $0 \mathrm{~W}$ CCP). Finally, the passivation layer resulting from the oxygen plasma was removed by plasma etching (SPTS Pegasus, $200 \mathrm{SCCM} \mathrm{CHF}_{3}, 35 \mathrm{SCCM} \mathrm{O}_{2}, 60$ s, 10 mTorr, $2500 \mathrm{~W}$ ICP, $100 \mathrm{~W}$ CCP, $20 \%$ LF pulse), and the standard Bosch etching was resumed to further etch the hole with a straight sidewall, as illustrated in Figure 4.2(d) and (e). By repeating these steps, holes with three big scallops at the sidewalls were created, as shown in Figure 4.1(a). The full procedure for creating holes with three big scallops is summarized in Table 4.2. 


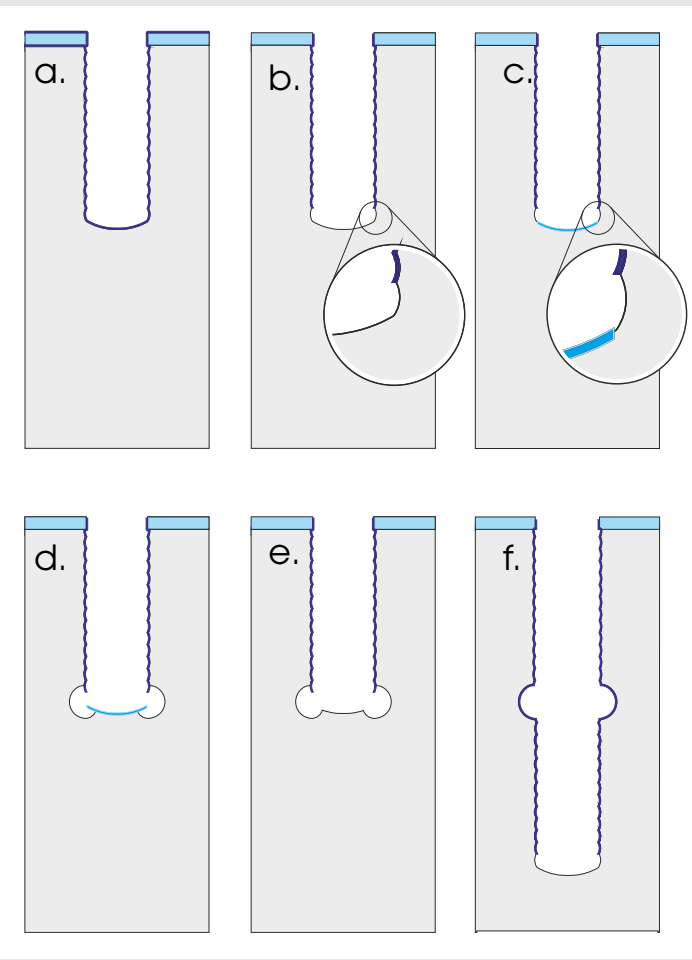

Figure 4.2: Illustration of the structures resulting from each step in the bottom passivation technique; dark blue represents fluorocarbon; the light blue material represents silicon oxide; (a) (structure after) last passivation step in the standard Bosch etching; (b) last etching step in the standard Bosch etching; (c) directional oxygen plasma treatment to selectively passivate the bottom surface; (d) isotropic plasma etching to develop a big scallop only from the sidewall; (e) selective removal of silicon oxide using $\mathrm{CHF}_{3} / \mathrm{O}_{2}$ plasma; (f) continuation of the standard Bosch process. 


\begin{tabular}{lrr} 
Parameters & Etch & Deposition \\
\hline Gas & $\mathrm{SF}_{6}$ & $\mathrm{C}_{4} \mathrm{~F}_{8}$ \\
Flow (SCCM) & 275 & 150 \\
Priority & 2 & 1 \\
Time (s) & 1.75 & 0.6 \\
Pressure (mTorr) & 26 & 20 \\
ICP (W) & 2200 & 2000 \\
CCP (W) & $20 \rightarrow 30$ & 0 \\
LF pulse & $20 \%$ & \\
average scallop height $(\mu \mathrm{m})$ & 0.3 & \\
Table 4. 1: Recipe for the standard Bosch process.
\end{tabular}

\subsubsection{Approach A: silicon nitride-based corner lithogra- phy}

The big scallops at the sidewall were used as concave corners for corner lithography to realize the sidewall masking. A $1 \mu \mathrm{m}$ thick layer of silicon rich nitride (SiRN) was first conformally deposited by low pressure chemical vapor deposition (LPCVD, $150 \mathrm{mTorr}, 77.5 \mathrm{SCCM}$ of $\mathrm{SiH}_{2} \mathrm{Cl}_{2}$, $20 \mathrm{SCCM}$ of $\mathrm{NH}_{3}$ and $150 \mathrm{SCCM}$ of $\mathrm{N}_{2}$ at $850^{\circ} \mathrm{C}, 4 \mathrm{~h}$, see Figure $4.3(\mathrm{~b})$ )), and then isotropically etched back in hot phosphoric acid (85 weight\%, $180^{\circ} \mathrm{C}$ ) for $7 \mathrm{~h}$ (Figure $4.3(\mathrm{C})$ ). Before etching, around $1.6 \mathrm{mg}$ of SiRN was dissolved into $3.5 \mathrm{I}$ etching solution to slightly saturate the solution and in that way decrease the etch rate from $6.9 \mathrm{~nm} \mathrm{~min}^{-1}$ (fresh solution) to approximately $3.3 \mathrm{~nm} \mathrm{~min}{ }^{-1}$. After the nitride etching, the mask formed by the nitride residues was inverted by performing local oxidation of silicon (LOCOS, wet oxidation, $1050^{\circ} \mathrm{C}, 30 \mathrm{~min}$ ) and stripping of the silicon nitride residue in hot phosphoric acid, as illustrated in Figure 4.3(d) and (e), to form the out-of-plane mask. Using this mask, three octahedra were etched at the locations of the big scallops in tetramethylammonium hydroxide (TMAH) solution $\left(70^{\circ} \mathrm{C}, 25\right.$ weight $\left.\%\right)$ for $45 \mathrm{~min}$, as illustrated in Figure 4.3(f). Figure 4.3(g) and (h) show the structure with repeated octahedra in cross-section and 3D view respectively. 


\begin{tabular}{|c|c|c|}
\hline repeating times & process & duration (s) \\
\hline 1 & oxygen strike & 5 \\
\hline \multirow{2}{*}{50} & $\mathrm{C}_{4} \mathrm{~F}_{8}$ deposition & 0.6 \\
\hline & $\mathrm{SF}_{6}$ etching & 1.75 \\
\hline \multirow{3}{*}{1} & directional oxygen plasma & 2 \\
\hline & isotropic $\mathrm{SF}_{6}$ etching & 5.2 \\
\hline & oxide removal & 60 \\
\hline \multirow{2}{*}{50} & $\mathrm{C}_{4} \mathrm{~F}_{8}$ deposition & 0.6 \\
\hline & $\mathrm{SF}_{6}$ etching & 1.75 \\
\hline \multirow{3}{*}{1} & directional oxygen plasma & 2 \\
\hline & isotropic $\mathrm{SF}_{6}$ etching & 5.6 \\
\hline & oxide removal & 60 \\
\hline \multirow{2}{*}{50} & $\mathrm{C}_{4} \mathrm{~F}_{8}$ deposition & 0.6 \\
\hline & $\mathrm{SF}_{6}$ etching & 1.75 \\
\hline \multirow{3}{*}{1} & directional oxygen plasma & 2 \\
\hline & isotropic $\mathrm{SF}_{6}$ etching & 6.0 \\
\hline & oxide removal & 60 \\
\hline \multirow{2}{*}{50} & $\mathrm{C}_{4} \mathrm{~F}_{8}$ deposition & 0.6 \\
\hline & $\mathrm{SF}_{6}$ etching & 1.75 \\
\hline
\end{tabular}

Table 4.2: Process for creating structures with three big isotropic scallops. 

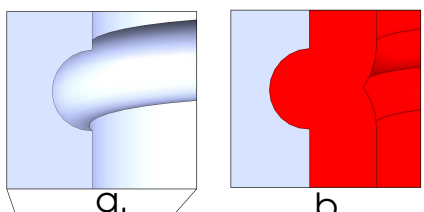

b.
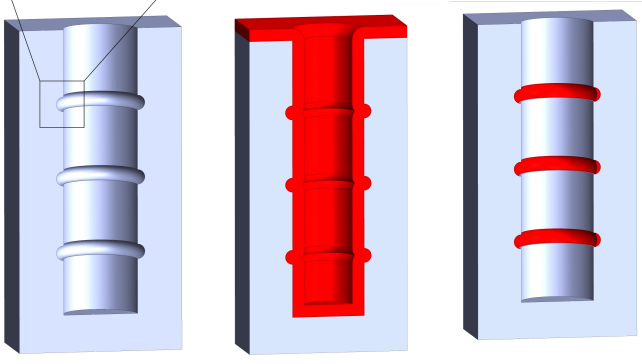

C.
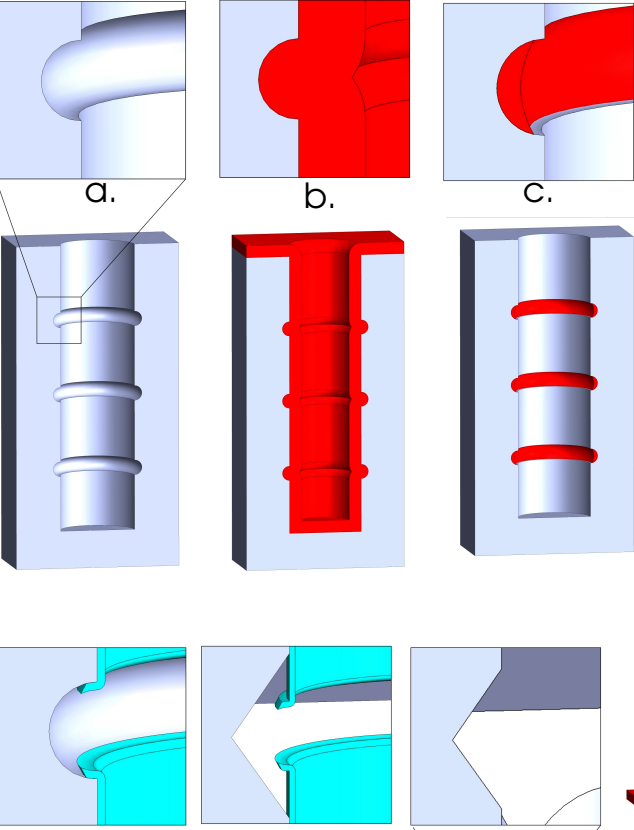

e.
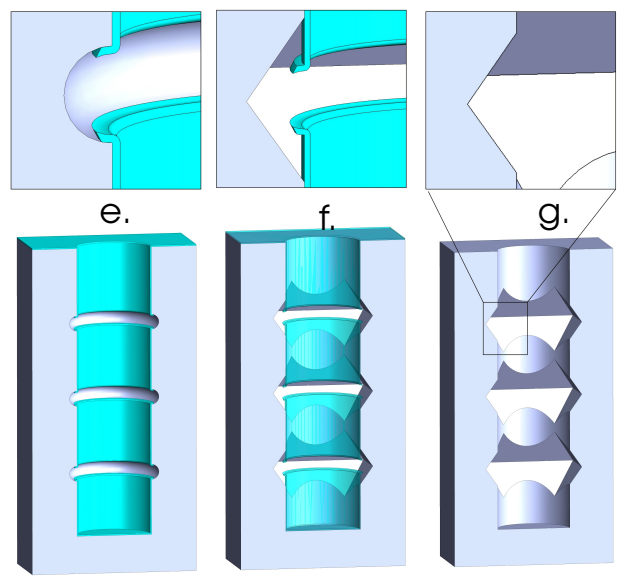

g.
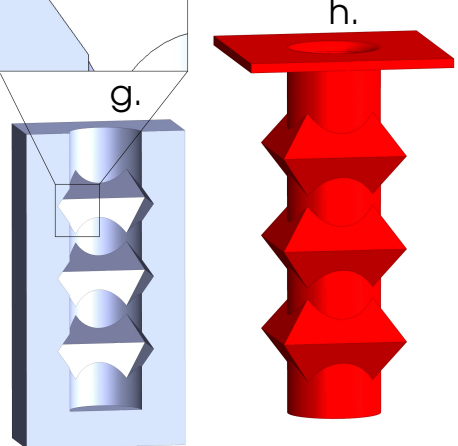

Figure 4.3: Out-of-plane masking and fabrication of repeated octahedra via the silicon nitride-based corner lithography route; (a) to (g) are cross-section views; (a) introduction of multiple big scallops; (b) conformal deposition of silicon nitride; (c) isotropic etching of silicon nitride to leave residues only inside the big scallops; (d) local oxidation; (e) stripping of the silicon nitride residue; (f) TMAH etching to introduce repeated octahedra; (g) structure with fully-developed octahedra; (h) $3 \mathrm{D}$ view of the structures with repeated octahedra. 

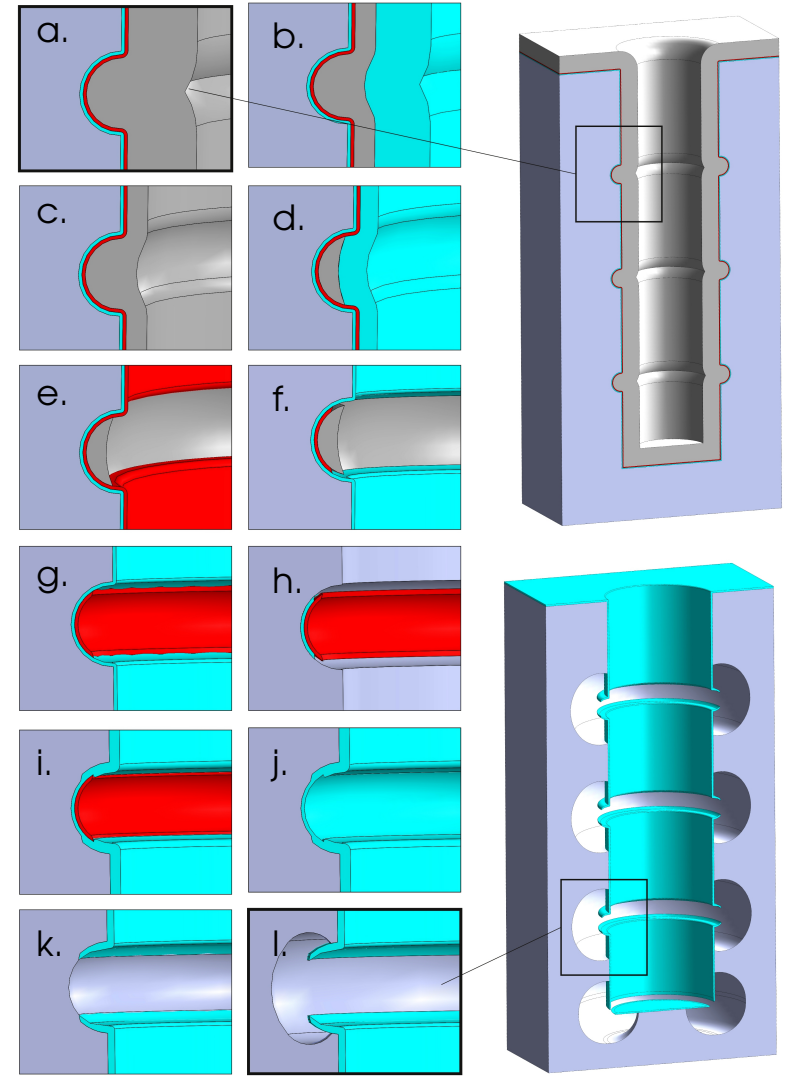

Figure 4.4: Illustration of the out-of-plane masking and fabrication of repeated octahedra by the combination of edge and corner lithography; (a) formation of silicon oxide/stoichiometric silicon nitride/polysilicon layer stack; (b-e) digital etching of the polysilicon by repeated oxidation and oxide stripping; (f) edge lithography of the nitride layer; ( $g$ ) stripping of the polysilicon residue; $(h)$ etching of the thin silicon oxide; (i) LOCOS; (j) stripping of the remaining nitride layer; (k) thinning of the oxide to open the mask; (I) silicon etching to form repeated octahedra or donuts. 


\subsubsection{Approach B: combination of digital etching-based corner lithography with edge lithography}

An alternative approach, which combines corner and edge lithography, was also tried. First, structures with big scallops were oxidized at $1050{ }^{\circ} \mathrm{C}$ (dry oxidation, $25 \mathrm{~min}$ ) to grow approximately $50 \mathrm{~nm}$ of oxide, followed by an oxide stripping process in 50 weight\% HF solution (30 s), to smoothen the sidewalls. Subsequently, another $50 \mathrm{~nm}$ of oxide was formed at $1050^{\circ} \mathrm{C}$ (dry oxidation, $25 \mathrm{~min}$ ). After the oxidation, $50 \mathrm{~nm}$ of stoichiometric silicon nitride (LPCVD, $150 \mathrm{mTorr}, 25 \mathrm{SCCM}$ of $\mathrm{SiH}_{2} \mathrm{Cl}_{2}, 250$ SCCM of $\mathrm{NH}_{3}$ and $200 \mathrm{SCCM}$ of $\mathrm{N}_{2}$ at $750^{\circ} \mathrm{C}, 48 \mathrm{~min}$ ) and $1.3 \mu \mathrm{m}$ of polysilicon (LPCVD, $250 \mathrm{mTorr}, 50 \mathrm{SCCM}$ of $\mathrm{SiH}_{4}$ and $250 \mathrm{SCCM}$ of $\mathrm{N}_{2}$ at $590^{\circ} \mathrm{C}$, $5 \mathrm{~h}$ ) were conformally deposited, as illustrated in Figure 4.4(a). Subsequently, digital etching ${ }^{94}$ was applied to leave polysilicon residues only inside the big scallops, as illustrated in Figure 4.4(b-e). Each digital etching step consists of thermal oxidation at $1050^{\circ} \mathrm{C}$ followed by selective oxide stripping in $50 \% \mathrm{HF}$ solution. Detailed information of the digital etching steps is shown in Table 4.3. By using this method, the size and shape of the residue are determined by thermal oxidation instead of by wet chemical etching. The sample was immersed in hot phosphoric acid (etch rate $3.8 \mathrm{~nm} \mathrm{~min}^{-1}$ ) for $45 \mathrm{~min}$ to retract the nitride layer below the polysilicon residue (edge lithography ${ }^{57}$ ), as illustrated in Figure 4.4(f). The polysilicon residue was stripped in TMAH solution, while the oxide layer underneath the nitride served as a protective layer for the bulk silicon, as illustrated in Figure 4.4(g). Subsequently, as shown in Figure 4.4(h) and (i), the oxide protective layer was retracted in 1 weight $\% \mathrm{HF}$ solution (room temperature, etch rate $4.6 \mathrm{~nm} \mathrm{~min}{ }^{-1}$ ) for $22 \mathrm{~min}$, and LOCOS (wet oxidation, $1050^{\circ} \mathrm{C}$ ) was applied to form $300 \mathrm{~nm}$ of silicon oxide on the sidewalls. After that, another $45 \mathrm{~s}$ of $1 \% \mathrm{HF}$ etching was performed to remove the oxidized outer surface of the silicon nitride, followed by stripping of the remaining nitride layer in hot phosphoric acid, as demonstrated in Figure 4.4(j). Finally, the sample was etched in $1 \% \mathrm{HF}$ solution for $36 \mathrm{~min}$ to thin down the oxide layer until the thinner oxide inside the big scallops was removed, to obtain the vertical mask (Figure 4.4(k)). As a demonstration of the principle, structures with repeated octahedra and rings were prepared by performing TMAH etching (45 min) and wet isotropic etching on the structures with this sidewall mask, as illustrated in Figure 4.3(h) and 4.4(i), respectively. 


\begin{tabular}{lll} 
Step & Treatment & $\begin{array}{l}\text { Thickness of resulting } \\
\text { oxide at (1 1 1) plane }\end{array}$ \\
\hline 1 & oxidation for $4 \mathrm{~h}$ & $910 \mathrm{~nm}$ \\
2 & stripping in $50 \% \mathrm{HF}$ for $45 \mathrm{~s}$ & $0 \mathrm{~nm}$ \\
3 & oxidation for $6 \mathrm{~h}$ & $1088 \mathrm{~nm}$ \\
4 & stripping in $50 \% \mathrm{HF}$ for $60 \mathrm{~s}$ & $0 \mathrm{~nm}$ \\
5 & oxidation for $6 \mathrm{~h}$ & $1088 \mathrm{~nm}$ \\
6 & storage in $800^{\circ} \mathrm{C}$ tube for $6 \mathrm{~h}$ & $139 \mathrm{~nm}$ \\
7 & stripping in $50 \% \mathrm{HF}$ for $60 \mathrm{~s}$ & $0 \mathrm{~nm}$ \\
8 & oxidation for $20 \mathrm{~min}$ & $316 \mathrm{~nm}$ \\
9 & stripping in $50 \% \mathrm{HF}$ solution for $20 \mathrm{~s}$ & $0 \mathrm{~nm}$ \\
& Table 4.3: Detailed information on the digital etching.
\end{tabular}

\subsubsection{Visualization}

In order to better visualize the silicon structures, samples with repeated octahedra were first etched in $50 \% \mathrm{HF}$ (room temperature) solution for $1 \mathrm{~min}$ to strip the oxide mask, and then a $\mathrm{Si}_{3} \mathrm{~N}_{4}$ layer of approximately $750 \mathrm{~nm}$ thick was deposited. Subsequently, the wafer was anodically bonded to a glass wafer, and the bulk silicon was removed from the back side in the TMAH solution to release the nitride negatives of the original silicon structures.

\subsection{Results and Discussion}

\subsubsection{Structure with highly isotropic big scallops}

The structure after the directional oxygen plasma and plasma etching without CCP (acceleration power) is shown in Figure 4.5(a), from which we can observe that a big scallop was etched only into the sidewall. This indicates that the oxygen plasma was highly directional so that the 
sidewall of the last small scallop remained exposed after the oxidation treatment. Figure 4.5(b) shows the structure after a straight sidewall was etched below the big scallop. There it can be seen that a nearly perfect half circle has been created with a horizontal depth of around $0.63 \mu \mathrm{m}$ and a vertical depth of around $1.14 \mu \mathrm{m}$. Figure $4.5(\mathrm{c})$ shows the big scallop resulting from a similar process where the formation and removal of the bottom passivation oxide was omitted. By comparing these two big scallops, we can find that the shape of the big scallop in Figure 4.5(b) is more rounded and the horizontal depth is larger. A deeper scallop leads to the formation of a larger residue during corner lithography, which means that there is more room for over-etching for removing residues from the straight parts of the sidewalls. Structures with three big scallops in the sidewall, created with the process as listed in Table 4.2, are shown in Figure 4.6.

The ear shape of the big scallop, as shown in Figure 4.5(c) was probably formed because the isotropic etching not only proceeded from the last small scallop, but also from the bottom surface, which has an inclination $\alpha$ close to the sidewall as shown in Figure 4.6. This angle resulted from the non-uniform physical bombardment of the bottom surface during the etching step of the Bosch process. The standard recipe uses sulfur fluoride $\left(\mathrm{SF}_{6}\right)$ to etch both fluorocarbon and silicon. Therefore, the protective layer around the center is removed more quickly and the silicon underneath starts to be attacked sooner than the silicon near the sidewalls. After numerous cycles, this effect will be magnified and results in the inclined angle. By applying the directional oxygen plasma, the bottom surface could not be etched during the isotropic plasma etching, and the inclined bottom surface near the edge therefore had no influence on the big scallop shape. Moreover, the passivated bottom also reduced the depletion of the etching species during the isotropic plasma etching step. In this way they were all available for the etching in the horizontal direction, which could also improve the isotropy.

\subsubsection{Approach A: silicon nitride-based corner lithogra- phy}

After the plasma etching process, silicon nitride-based corner lithography was performed. Figure 4.7(a) displays the profile after the con- 

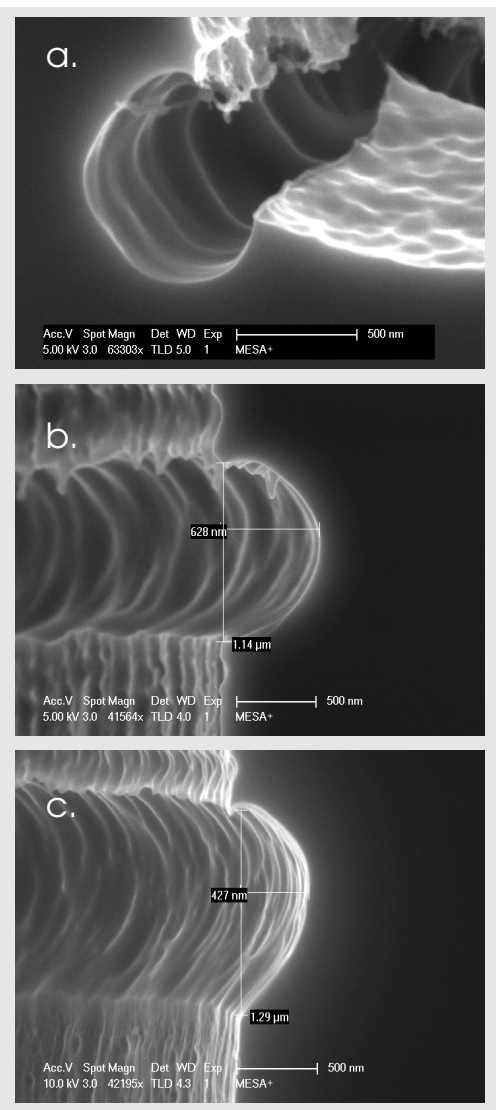

Figure 4.5: (a) Big isotropic scallop introduced after the directional oxygen plasma treatment and isotropic plasma etching; (b) isotropic big scallop created using the bottom passivation technique after further etching of the straight sidewall; (c) big scallop created without the directional oxygen plasma treatment. 


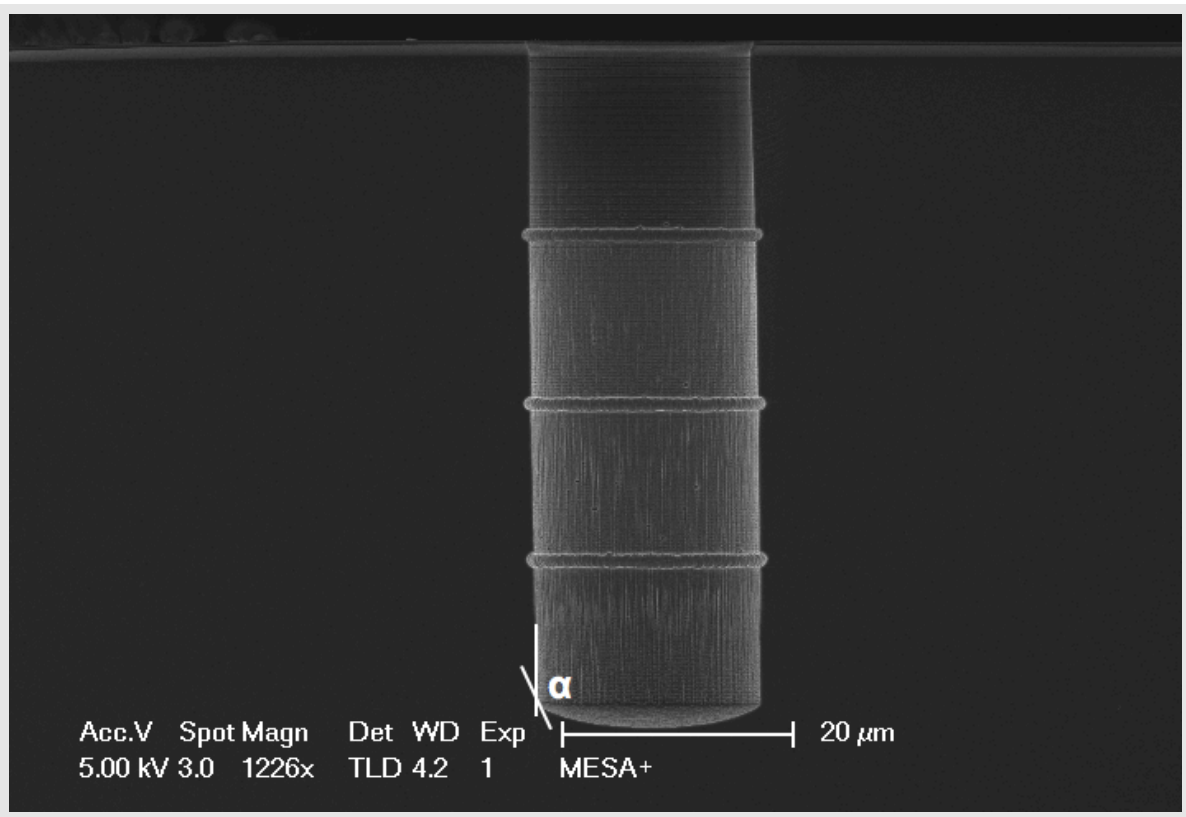

Figure 4.6: Structure with three big scallops created using the bottom passivation technique.

formal deposition, from which we can see that the outermost surface of the nitride layer is nearly flat. Subsequently, the sample was etched in the pretreated hot phosphoric acid solution for $7 \mathrm{~h}$ in one run, and the resulting profile is displayed in Figure 4.7(b) and (c). There it can be seen that the nitride residues only remain inside the big scallops. Before etching, SiRN was dissolved in the etching solution to reduce the chemical reaction rate so that the nitride etching process is more chemical reaction-limited instead of diffusion-limited, which is beneficial for improving the etching uniformity. Without this etch speed reduction a finite nitride layer or residue was left on the sidewalls deeper inside the hole, which led to defects or even complete sidewall erosion in the following TMAH etching process. To form the out-of-plane mask, LOCOS and residue stripping were performed. Using this mask, structures with repeated octahedra were formed, as shown in Figure 4.7(d). The released nitride negatives are displayed in Figure 4.7(e) and (f), from which we can see that structures with repeated octahedra have 
been uniformly introduced over large areas.

\subsubsection{Approach B: combination of digital etching-based corner lithography with edge lithography}

The success of the silicon nitride-based corner lithography depends on the uniformity of the nitride etching. Despite the pretreatment of the etching solution, etch speed differences were found between the top and bottom of high aspect ratio structures. For higher aspect ratio structures this could have the result that the topmost residues are completely etched before the sidewalls at the bottom are free of nitride. To make the process less dependent on the etching uniformity, an alternative approach, which combines corner and edge lithography ${ }^{57}$, was also tried. Figure 4.8(a) shows that the layers have the designed thickness everywhere. The structure after the digital etching is displayed in Figure 4.8(b), where we can see that the straight part of the sidewall is already free of polysilicon, and the thickness of the residue inside the big scallop is around $450 \mathrm{~nm}$. However, corner lithography was also unintentionally performed at the bottom corner of the hole, leaving a residue of about $370 \mathrm{~nm}$, as shown in Figure 4.8(c), which is caused by the rounding effect of thermal oxidation.

Edge lithography was performed by etching the stoichiometric silicon nitride, retracting it below the polysilicon residues, as shown in Figure 4.8(d). The edge lithography further widens the over-etching window, as the outer surface of the remaining nitride layer is protected by the residue, and the etchant can only remove the material through the edge of the polysilicon residue. This over-etching would lift off small unwanted polysilicon residues at the sidewall. After the edge lithography of silicon nitride, the polysilicon residue was stripped, and the oxide protective layer was etched in $1 \% \mathrm{HF}$ solution using the remaining nitride as a mask. The structure after the $1 \% \mathrm{HF}$ etching is displayed in Figure 4.8(e). Subsequently, LOCOS was applied and the resulting structure is shown in Figure 4.8(f), from which we can see that the thickness of the oxide that was below the silicon nitride was greatly increased, forming a significant 'bird's beak' below the silicon nitride. This significant growth of pad oxide is probably related to the undercut of the pad oxide, as shown in Figure 4.8(e), so that oxygen can diffuse more easily under the nitride during the LOCOS step. However, this 

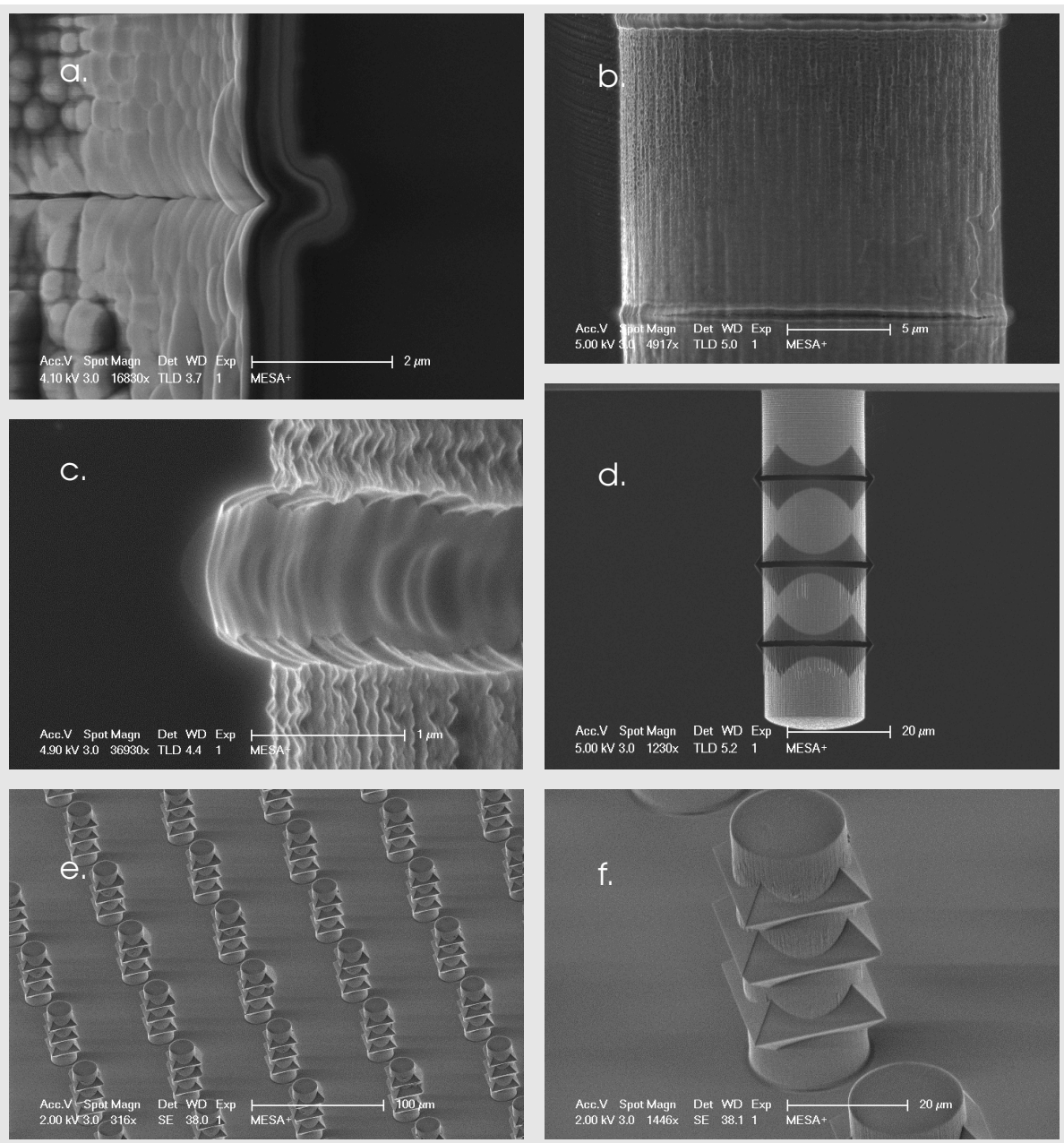

Figure 4.7: (a) Profile of a big scallop after conformal deposition of a SiRN layer of $1 \mu \mathrm{m}$ thick; $(b, c)$ structure after the etching in the modified phosphoric acid solution ; (d) cross-sectional view of the structures with repeated octahedra; $(e, f)$ released pillar structures made inside the silicon structures created using the silicon nitride-based corner lithography. 

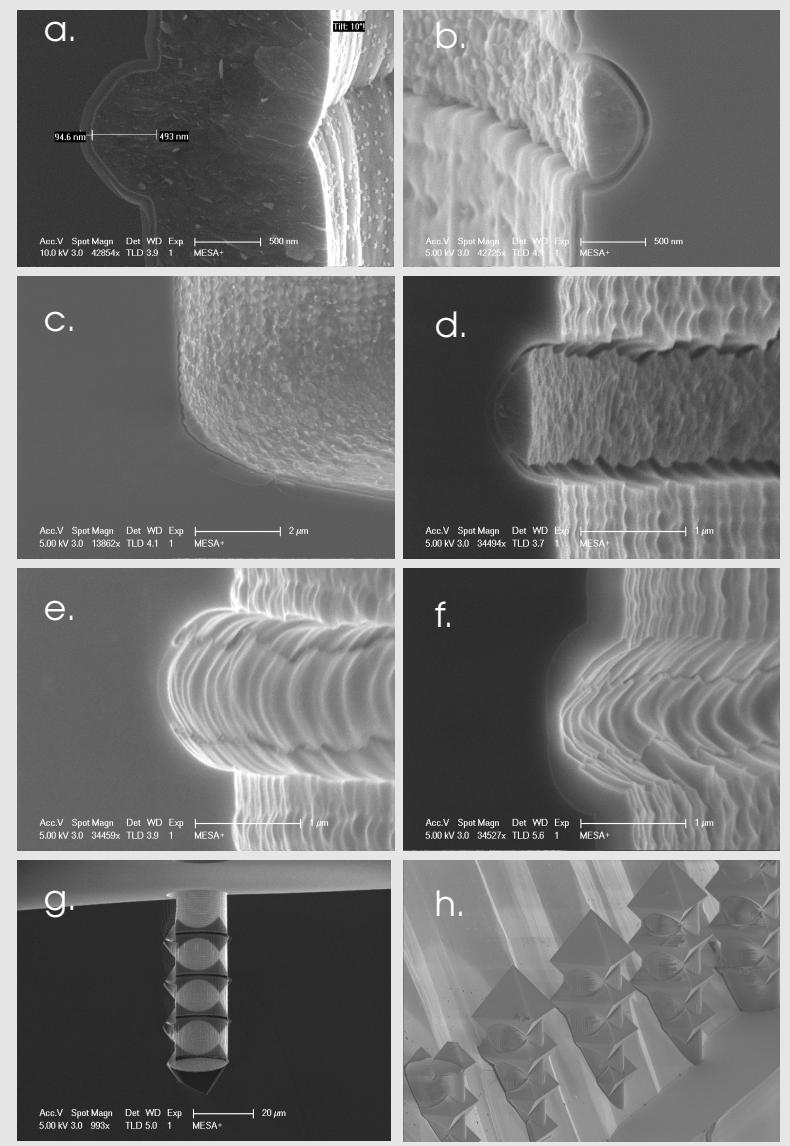

Figure 4.8: (a) Layer stack for digital etching and edge lithography in a big scallop; (b) profile of the big scallop after digital etching; (c) profile of the bottom corner after digital etching; (d) structure after retraction of the stoichiometric nitride layer along the edge of the polysilicon residue; (e) structure after the retraction of the thin oxide layer in $1 \%$ HF solution; (f) profile of the big scallop after LOCOS; (g) crosssectional view of the structure with repeated octahedra resulting from the combination of corner and edge lithography; $(h)$ pillars with repeated octahedra resulting from the combination of corner and edge lithography. 
may be beneficial for the elimination of sidewall defects as remaining small nitride residues at the sidewall can be easily lifted off due to the significant oxide growth. Finally, timed etching of the oxide in $1 \% \mathrm{HF} \mathrm{so-}$ lution was performed to open the oxide inside the big scallops to form the vertical mask. As a demonstration, repeated octahedral structures were etched using this mask, as shown in Figure 4.8(g). Due to the residues left at the bottom corner, an extra octahedron was formed over there. The partially released pillars are shown in Figure 4.8(h), from which we can see that the four octahedra were introduced along the length of the pillar.

Based on the vertical mask, various complicated structures, such as repeated rings and fractals, can be introduced by applying different combinations of isotropic and anisotropic etching and corner lithography, as shown in Figure 4.9. In this way, the feature density can be unprecedentedly increased as the features could self-align in both horizontal and vertical directions.

\subsection{Conclusion}

In this Chapter, a technique that combines plasma etching and corner lithography was shown to be usable for wafer-scale out-of-plane patterning. DRIE was used to create cavities with multiple big semicircular scallops at their sidewalls. These were used as concave corners for corner lithography, leaving residues only inside these big scallops, to form a vertical masking pattern. As an example to demonstrate the potential of this technology in the fabrication of repeating 3D structures, various structures, like octahedra and donuts, were repeated in the vertical direction.

To achieve a better shape of the big scallops, a bottom passivation process was introduced into the Bosch process. It consists of a bottom passivation step, which uses directional oxygen plasma to selectively oxidize the bottom surface, an isotropic, radical-dominated plasma etching process to develop the big scallop from the sidewall of the last small scallop, and a bottom removal step, which uses $\mathrm{CHF}_{3} / \mathrm{O}_{2}$ plasma to selectively remove the passivation layer. In this way, the influence of the inclination angle between the sidewall and the bottom surface is eliminated, and the depletion of etching species is reduced. 

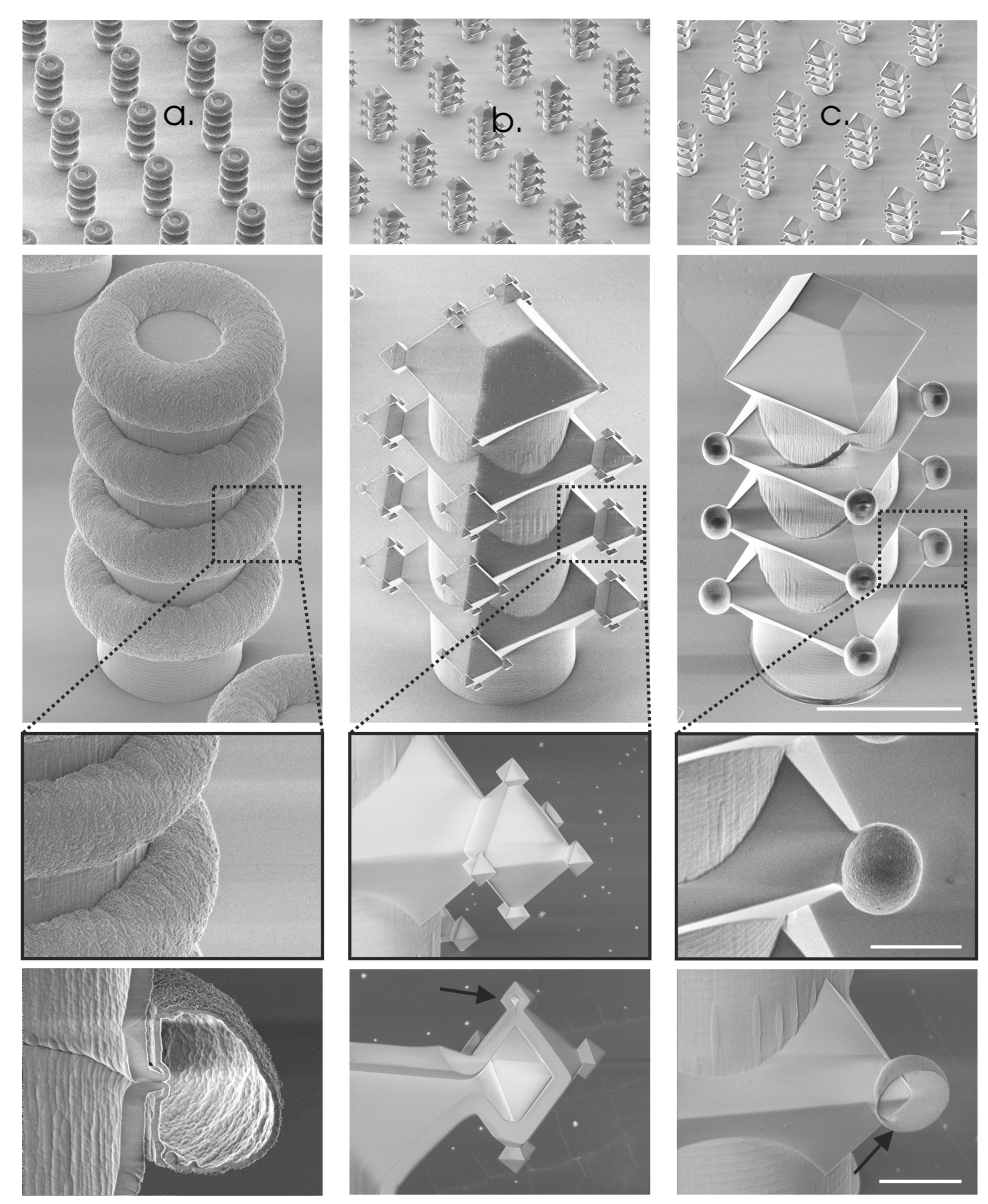

Figure 4.9: Various structures made using the repeated vertical mask, by applying (a) isotropic etching, (b) three times anisotropic etching interchanged with corner lithography ${ }^{67}$ or (c) anisotropic etching, corner lithography and isotropic etching. Scale bars in the top two rows are $20 \mu \mathrm{m}$ and $5 \mu \mathrm{m}$ in the bottom two rows. 
As a result, big scallops with nearly perfect isotropy are created for the first time by DRIE.

Corner lithography was used to create a vertical mask based on these sidewalls with a few bigger scallops. This technique leaves residues in the concave corners formed by these big scallops, and this pattern is transformed into a sidewall mask using LOCOS. Silicon nitride-based corner lithography is relatively simple, as it involves fewer steps in comparison with a process that combines edge lithography with digital etching-based corner lithography. The latter approach is less sensitive to etching uniformity and sidewall defects.

Overall, the combination of multiple well-formed big scallops in the sidewalls with either corner lithography technique was shown to be suitable for the formation of a mask to create repeated structures in the out-of-plane direction, using conventional wafer-scale microfabrication techniques. In the future this might be useful for example for increasing the feature density in integrated circuit technology, for covering large surfaces with structures with special optical properties, 3D microfluidic networks/mixers/filters, and solar water splitting devices. 


\section{Chapter 5}

\section{A photocathode: radial junction silicon microwires with spatioselectively deposited catalyst}

This chapter is based on "Spatial Decoupling of Light Absorption and Catalytic Activity of Nickel-Molybdenum on High-Aspect-Ratio Silicon Microwire Arrays", Vijselaar et al. ${ }^{95}$.

A good solar-to-fuel device is efficient both in light harvesting and in transforming the photogenerated electricity into chemical bonds. Silicon (Si) is a popular, high-performing photon absorber. However, Si as a photocathode has poor kinetics for the hydrogen evolution reaction (HER) ${ }^{96}$ and therefore requires a catalyst to achieve efficient solar-to-hydrogen conversion. Unfortunately, however, for state-of-theart earth-abundant catalysts (such as Ni-Mo), the high mass loading that is needed for sufficient catalytic activity also results in considerable parasitic light absorption upon front side illumination (as in Type C, see Section 8. 1), which reduces the efficiency of such photocathodes.

One promising route to obtain high efficiencies for systems consisting of semiconductors coated with a catalytic material is by spatially and functionally decoupling the optical absorption and the catalytic 
activity of the photocathode. Silicon microwire arrays provide an approach to overcome the negative correlation between the catalytic activity, which is directly related to the fill factor, $F F$, in terms of photovoltaic (PV) cells, and light absorption, $J_{\mathrm{ph}}$. High $F F$ values in Si microwire photocathodes have been obtained by covering the bottom area between the microwires with $\mathrm{Ni}-\mathrm{Mo} / \mathrm{TiO}_{2}{ }^{97}$ or cobalt phosphide nanoparticles ${ }^{97,98}$. The best-performing Si homojunction microwire array photocathodes from these studies demonstrated an ideal regenerative cell efficiency, $\eta_{\mathrm{IRC}}$, of 2.9 and $2.8 \%$ respectively ${ }^{97-99}$. These efficiencies, obtained in designs without surface passivation, were restricted by light absorption, evidenced by a limited photocurrent $(<$ $15 \mathrm{~mA} \mathrm{~cm}^{-2}$ ), whereas passivated Si PV cells easily generate about 40 $\mathrm{mA} \mathrm{cm}^{-2}$ 100,101. Surface passivation suppresses recombination of charge carriers at the surface. This becomes increasingly important as the absolute surface area increases, which is the case especially for highaspect-ratio structures.

By studying ultramicroelectrode arrays of platinum (Pt) disks on $\mathrm{n}^{+}$$\mathrm{Si}$, it was found that placing catalyst islands as far as $12 \mu \mathrm{m}$ apart did not significantly increase the kinetic overpotential of the HER, but that, beyond that distance, catalyst performance went down 102. Although this study was limited to a two-dimensional (2D) surface, it is clear that the spacing of separate catalyst islands can strongly influence the catalyst performance of any photocathode. Because this might also hold for the 3D photocathodes discussed in the present paper, we investigate the influence of microwire pitch (center-to-center distance between the microwires) on performance of these devices.

Here we describe the spatial and functional decoupling of light absorption and catalytic activity of front side-illuminated Si microwire array photocathodes. Radially doped microwire arrays are passivated by $\mathrm{SiO}_{2}$, which increases the efficiency of the underlying PV cell6,101,103. The $\mathrm{SiO}_{2}$ is spatioselectively removed from the top sides of the microwire arrays. The remaining, insulating $\mathrm{SiO}_{2}$ layer acts as a masking layer in a subsequent process step in which the freshly exposed Si top is covered with an electrodeposited catalyst for $\mathrm{H}_{2}$ production. The influence of the size of the catalyst-covered region and the spacing between the microwires on the performance of the resulting photocathodes was investigated, by measuring their photovoltaic $(J-V$, where $J$ is current density and $V$ voltage), electrochemical and photo-electrochemical $(J-E)$, where $E$ is potential versus RHE) characteristics. Variation and

80 
comparison of the different device parameters results in a deeper understanding of the limiting factors in photocathode performance, leading to an optimized design with an ideal regenerative cell efficiency, $\eta_{\mathrm{IRC}}$, of $>10 \%$.

\subsection{Results}

\subsubsection{Spatioselective coating of $\mathrm{Ni}-\mathrm{Mo}$ on microwires}

Substrates with arrays of silicon microwires $(4 \mu \mathrm{m}$ diameter, $40 \mu \mathrm{m}$ length and $6 \mu \mathrm{m}$ pitch) with radial junctions (p-type base and $900 \mathrm{~nm} \mathrm{n}^{+}$-type emitter) were fabricated according to a procedure reported previously ${ }^{6}$. The microwire length was chosen to provide optimal light absorption ${ }^{103}$. The substrates were processed in one of three ways. To deposit a catalyst over the entire surface of the Si microwire arrays (Figure 5.1(a)), the arrays were subjected to electrodeposition without further preparation. For selective deposition near the tops of the microwires (Figure 5.1(b)), the wires were first conformally covered with $\mathrm{SiO}_{2}$, followed by a photoresist layer, which was etched back in $\mathrm{O}_{2}$ plasma to expose the tops of the wires. Subsequently, the exposed $\mathrm{SiO}_{2}$ layer was etched in buffered hydrogen fluoride (BHF) (see Section 5.3.3 for details). For the electrodeposition of catalyst that covered more of the microwires from the top down, we used selective removal of $\mathrm{SiO} 2$ from the tops of the microwires (with the method described in Section 3.2 to the desired height $X$, as measured from the top of the microwire (Figure 5.1(c)).

Homogeneous electrodeposition of bright nickel (Ni) over 3D microstructures, such as Si microwires, has been described before ${ }^{104}$. The addition of a relatively low concentration of $\mathrm{Na}_{2} \mathrm{MoO}_{4}(24 \mathrm{mM})$ to the $\mathrm{NiSO}_{4}(1.3 \mathrm{M})$ deposition bath, to form Ni-Mo, alters the composition and growth morphology of the layer 80,105 . High-resolution scanning electron microscope (HR-SEM) images reveal that electrodeposition of $\mathrm{Ni}-\mathrm{Mo}$ in the range of 10 to $180 \mathrm{~s}$ does not lead to a continuous thin film on bare Si microwire arrays; instead, isolated particles are deposited all over the surface (Figure 5.2). The difference in growth morphology between the two materials (Ni versus Ni-Mo) is attributed to the low current efficiency, which is below $5 \%$ in the case of $\mathrm{Ni}$-Mo deposition, as 


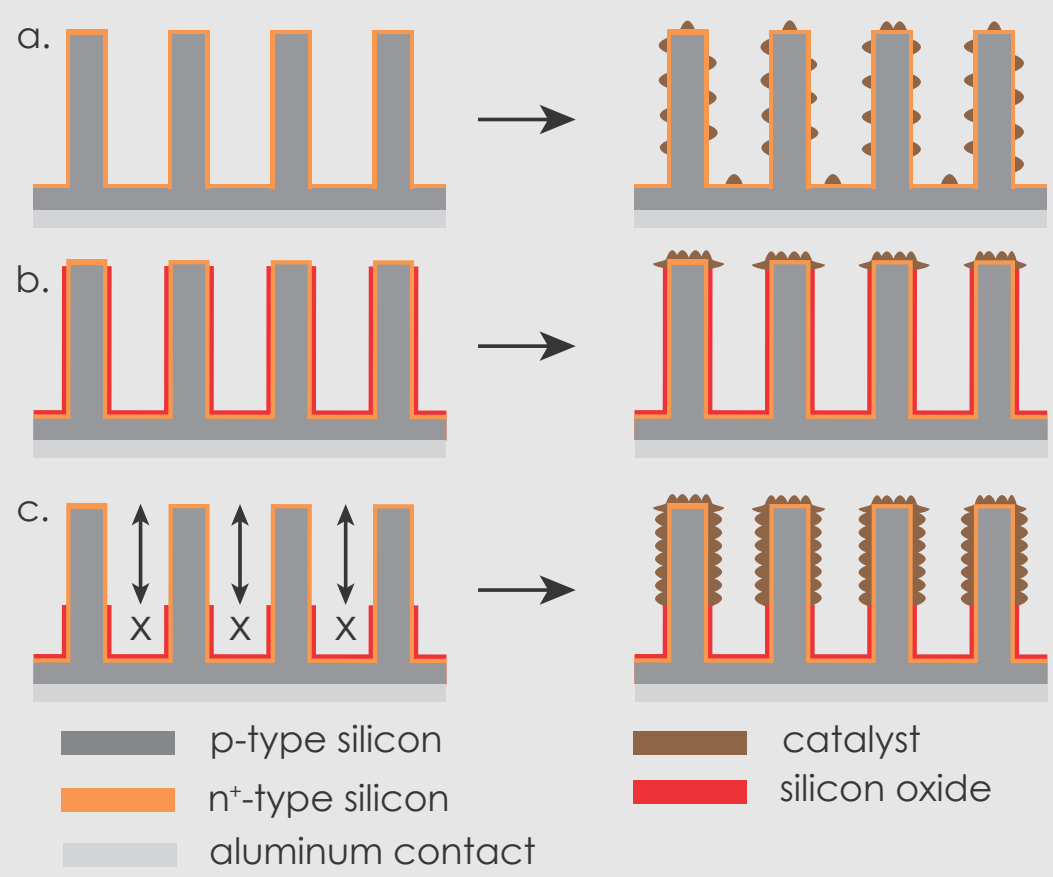

Figure 5.1: Electrodeposition of earth-abundant catalysts, Ni-Mo, on Si microwire arrays with a radial junction. Fabrication process for completely exposed microwires (a), microwires passivated by $\mathrm{SiO}_{2}$ with exposed tops (b), or samples with a defined exposed area in the range of 2-36 $\mu \mathrm{m}$ from the top (c)

compared with nearly $100 \%$ under the conditions for Ni deposition from a sulfate solution 106-110. Furthermore, it was observed that the Ni-Mo particles at the tops of the microwires agglomerate slightly, whereas, at the bottom, individual particles are evenly distributed over the surface.

The crystal structure of the deposited Ni-Mo films was confirmed by X-ray diffraction (XRD) (Figure 5.3) ${ }^{111}$. Films of Ni-Mo were compared with electrodeposited $\mathrm{Ni}$ films and a reference pattern, and it was found that both the (111) and (002) peaks of Ni-Mo were shifted relative to the Ni pattern ${ }^{112}$. This is in line with reports that Mo cannot be 

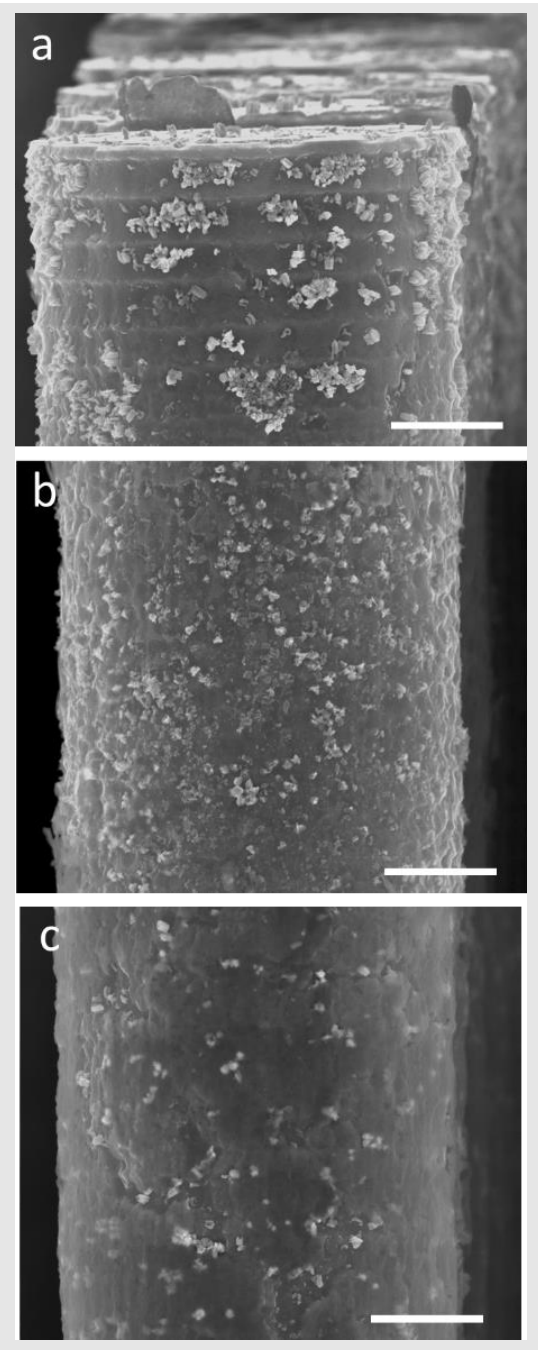

Figure 5.2: HR-SEM images from (a) top, (b) middle, and (c) bottom section of a single micropillar which was completely exposed to $30 \mathrm{~s}$ electrodeposition of Ni-Mo (sample A); scale bar is $1 \mu \mathrm{m}$. 
deposited as a pure metal from an electrodeposition bath and only occurs in co-deposition with another metal such as nickel ${ }^{108-110}$. Previous research ${ }^{112}$ indicated that this Ni-Mo alloy has 9 atomic percent of molybdenum incorporated in the structure.

Figure 5.4(a) presents an HR-SEM image of a Si microwire with high mass loading of Ni-Mo solely at the upper $\sim 2 \mu \mathrm{m}$, and Figure 5.4(b) shows a zoom-out of the corresponding microwire array. The results confirm that, during the electrodeposition process, the insulating layer of $\mathrm{SiO}_{2}$ effectively inhibits the transfer of electrons at the wire surface area below the exposed tops, thereby restricting catalyst deposition to the top side of the microwire arrays. Similar to what was observed for Ni-Mo deposition on the samples in Figure 5.2, the electrodeposition process gave closely packed agglomerated particles, but did not completely cover the exposed silicon. Owing to the smaller exposed areas, for equal deposition times denser Ni-Mo particle films were formed on the top-exposed wires (Figure 5.4(a)) than on fully exposed wires (Figure 5.2). As a limitation of our photocathode, it has to be noted that owing to the open, granular structure of the electrodeposited Ni-Mo (see Figure 5.4(a)), the Si microwire arrays with spatioselectively deposited Ni-Mo are not stable in alkaline electrolyte solutions.

\subsubsection{Photoelectrical (J-V) measurements}

The microwire arrays with a radial $\mathrm{n}^{+} / \mathrm{p}$-junction were also fabricated as a solid-state Si PV cell, as described previously6,101,103. A schematic representation of the set-up is given in Figure 5.5. This allows the effects of the addition of Ni-Mo on the light-harvesting capabilities to be assessed, without interference from other factors, such as electrolyte composition, bubble formation or contact resistances. We performed $J-V$ measurements on both bare and catalyst-coated Si microwire arrays with fully (sample type $A$ ) or partially (sample type B) exposed wires (Figures 5.6 and 5.7). Characteristic values of open-circuit potential $\left(V_{\text {oc }}\right)$, short-circuit current $\left(J_{\text {sc }}\right), F F$ and efficiency $(\eta)$ are given in Table 5.1.

For samples of fully exposed wires (A), the addition of $\mathrm{Ni}-\mathrm{Mo}$ as a catalyst did not significantly influence the $F F$ or $V_{\text {oc }}$ of the $J-V$ curve (see Figure 5.6 and Table 5.1). It solely blocked and/or scattered the 


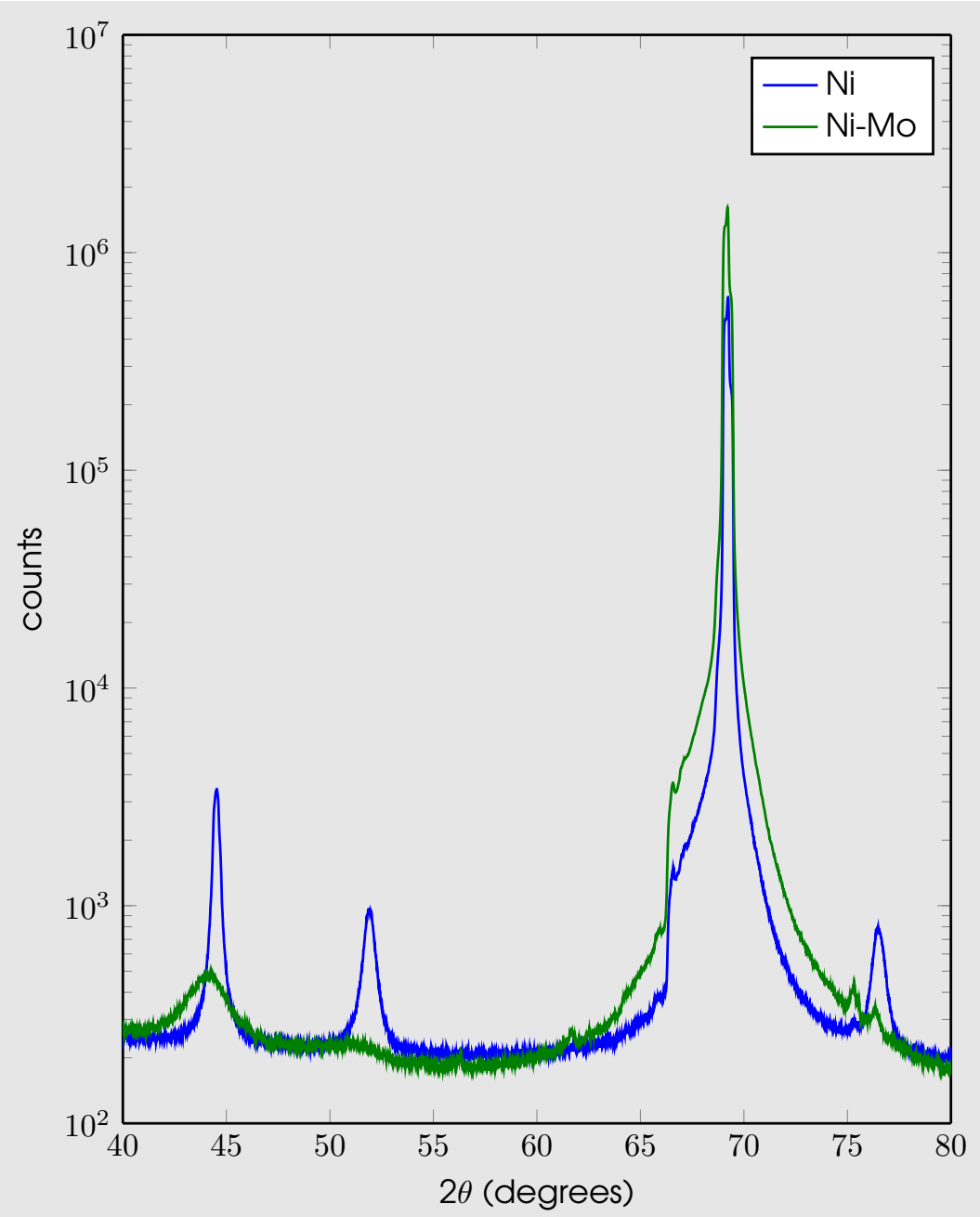

Figure 5.3: XRD patterns of electrodeposited nickel (30s) and alloyed nickel molybdenum films (180 s) on flat silicon. For nickel the (11 1), (002), and (022) peaks are observed at $44.56^{\circ}, 51.92^{\circ}$, and $76.50^{\circ}$, respectively, and no interfacial phases. For Ni-Mo they are shifted, at $43.96^{\circ}, 51.21^{\circ}$, and $75.35^{\circ}$ respectively, confirming the incorporation of Mo atoms in the nickel structure. The high intensity peaks at $69.13^{\circ}$ in both spectra are $\mathrm{p}-\mathrm{Si}(400)$, and their coincidence confirms the alignment of both samples. 

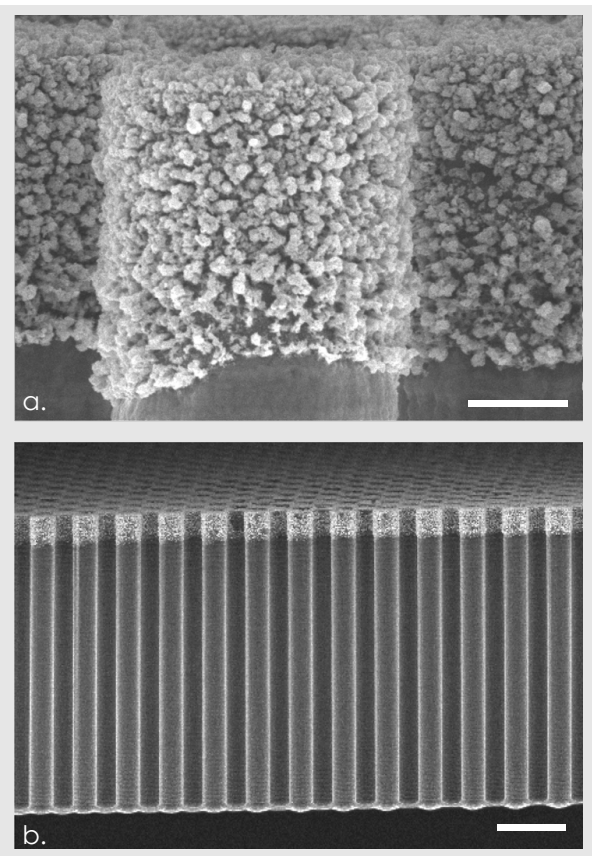

Figure 5.4: Electrodeposition of earth-abundant catalysts, Ni-Mo, on Si microwire arrays with a radial junction. HR-SEM images of Si microwire arrays with $n+/ p$ junctions and Ni-Mo solely on the tops of the microwire (sample B, 180 s deposition); scale bars are $1 \mu \mathrm{m}$ (a) and $10 \mu \mathrm{m}$ (b).

light, as a consequence of which the $J_{\mathrm{sc}}$ rapidly decreased for increasing $\mathrm{Ni}$-Mo deposition time. When the deposition time was increased to $180 \mathrm{~s}$, little light was converted into photocurrent, and the efficiency dropped to $1.1 \%$. A deposition time of $180 \mathrm{~s}$ was used because it gave the highest catalyst activity (Figure 5.8) for $J$-E measurements in the dark.

For the Si microwire arrays in B samples, for which approximately the upper $1 \mu \mathrm{m}$ of $\mathrm{SiO}_{2}$ was spatioselectively removed and covered with $\mathrm{Ni}-\mathrm{Mo}$, the $\mathrm{SiO}_{2}$ not only forms an electrical barrier that prevents catalyst deposition during the electrodeposition step, it also passivates the Si surface against charge carrier recombination and thereby enhances the PV efficiency ${ }^{101,113-115}, J-V$ measurements performed after 


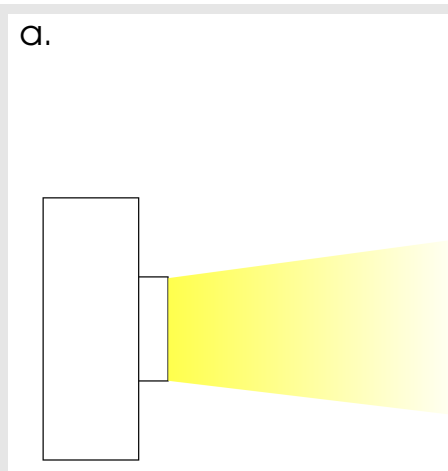

Light Source

b.

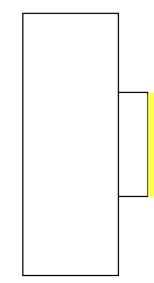

Light Source
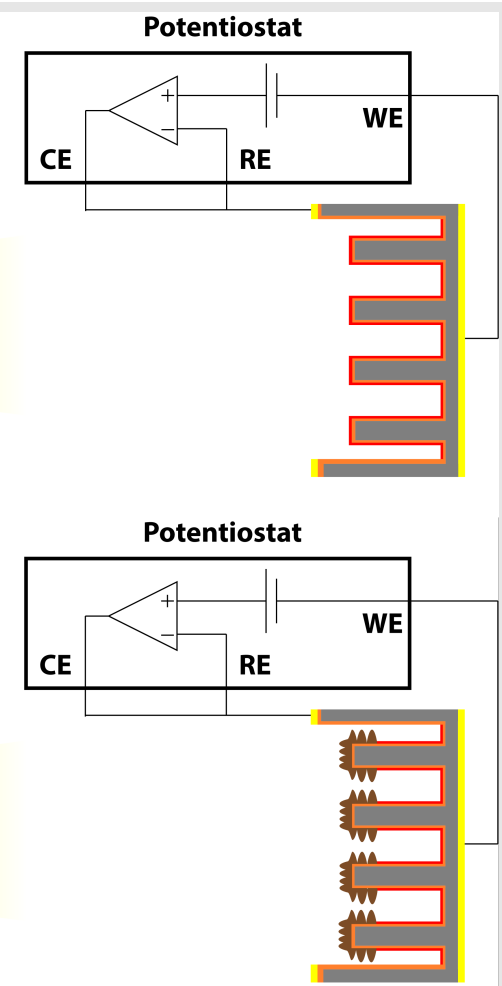

Figure 5.5: A schematic representation of how the potentiostat is connected to the samples to measure specific elements of the photocathode. CE is the counter electrode, RE is the reference electrode, and $W E$ is the working electrode. (a) Light $J$ - $V$ measurement setup for fully passivated microwire PV cells. (b) Light $J-V$ measurement setup for spatioselectively catalyst-functionalized microwire PV cells. The sample is placed at 1 sun intensity (see Section 5.3.8 for calibration details) and the potential is scanned linearly between -0.7 and $0.7 \mathrm{~V}$, while the RE and CE are connected together at the front side and the WE is connected at the backside. 


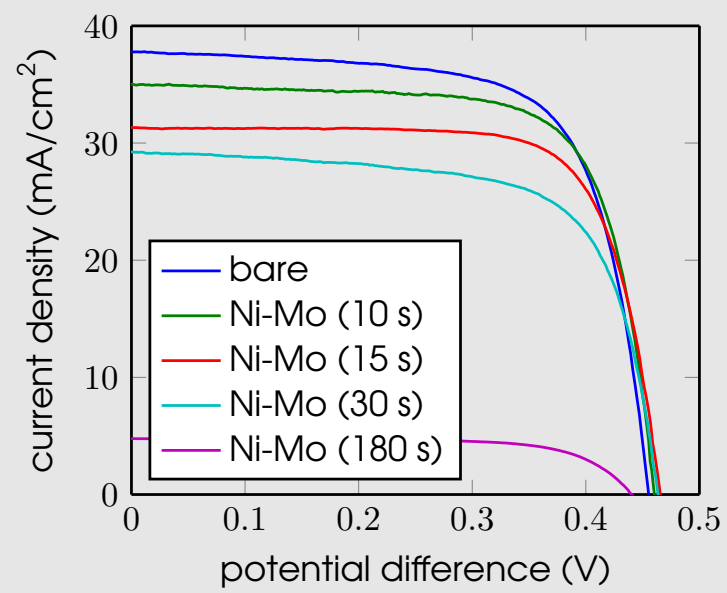

Figure 5.6: $J-V$ measurement of photocathodes with and without $\mathrm{Ni}$ Mo, using the deposition times shown, on fully exposed Si microwires, using AM 1.5G illumination. Each line is the average of at least four different samples prepared with the same settings.

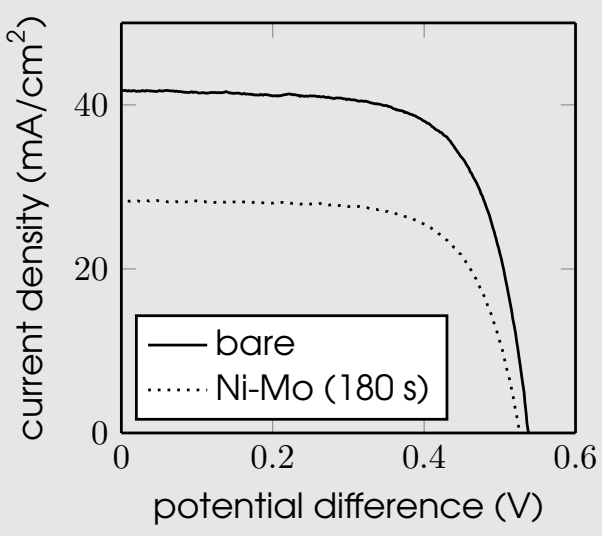

Figure 5.7: $J-V$ measurement of photocathodes with and without $\mathrm{Ni}$ Mo electrodeposited for $180 \mathrm{~s}$ spatioselectively on top-exposed arrays, using AM 1.5G illumination. Each line is the average of at least four different samples prepared with the same settings. 


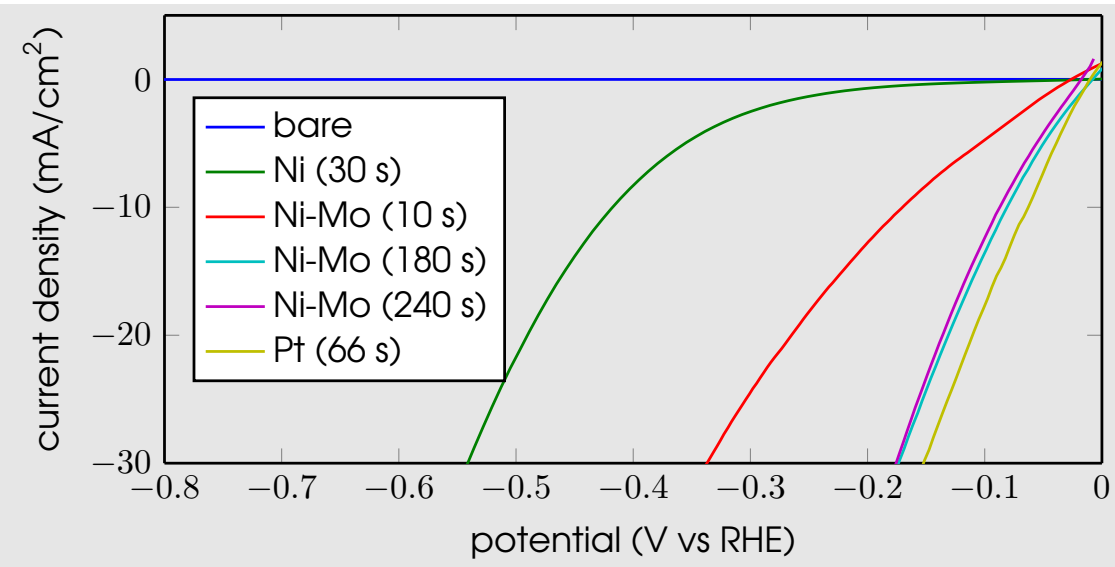

Figure 5.8: Dark $J-E$ behavior for HER electrocatalysis on $\mathrm{n}^{+}-\mathrm{Si} \mathrm{mi}-$ cropillar array substrates, without or completely covered with a catalyst of $\mathrm{Ni}$, Ni-Mo and $\mathrm{Pt}$ (sample A). All samples were evaluated in $0.1 \mathrm{M} \mathrm{H}_{2} \mathrm{SO}_{4}(\mathrm{aq})$. The used setup to measure the activity is given in Figure $5.9(\mathrm{~b})$. The band edge position of $\mathrm{Si}$ straddles the $\mathrm{H}^{+} / \mathrm{H}_{2}$ redox couple, and almost no catalytic activity is observed over a large overpotential range for bare Si micropillars, which underlines the requirement for a catalyst. The addition of a smooth, continuous Ni film on the surface already improves the catalytic activity substantially ( $\mathrm{Ni}(30 \mathrm{~s})$ ). A relatively small addition of $\mathrm{Na}_{2} \mathrm{MoO}_{4}(<2$ mole percent $\mathrm{Mo}$ to $\mathrm{Ni}$ ) to the plating bath solution increases the HER activity in an acidic environment even more. Such a Ni-Mo alloy on $\mathrm{n}^{+}-\mathrm{Si}$ micropillar arrays gives $J-E$ responses that exhibit increasing catalytic activities with increasing deposition time. Increasing the deposition times beyond $180 \mathrm{~s}$ does not have any further effect, as is seen by increasing the deposition time to $240 \mathrm{~s}$. Furthermore these layers show a catalytic activity similar to that of Pt. 


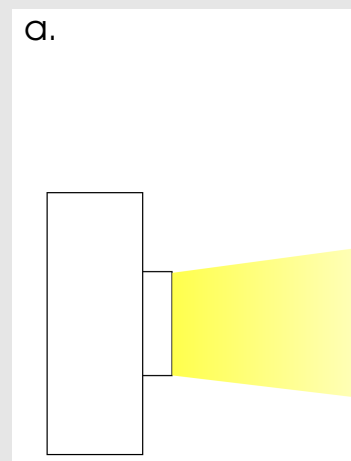

\section{Light Source}

b.

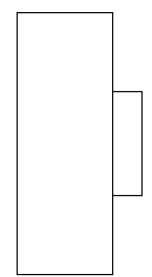

\section{Light Source}

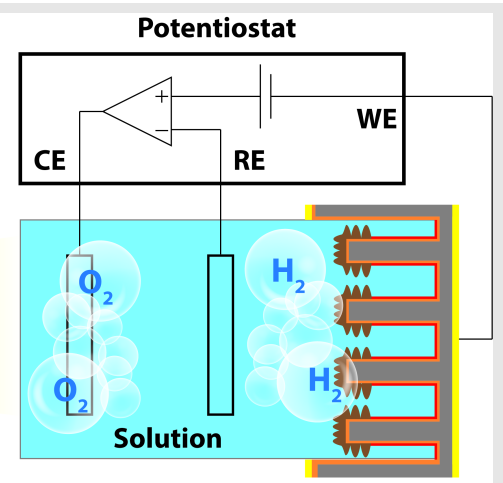

Potentiostat

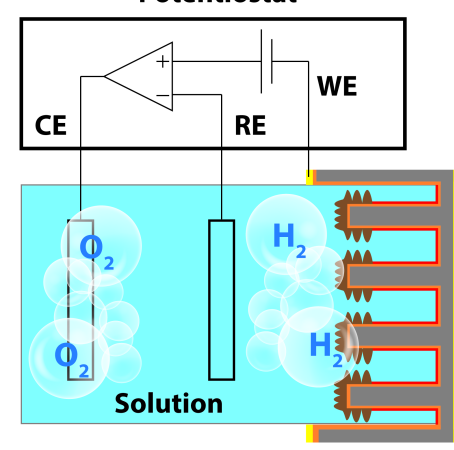

Figure 5.9: A schematic representation of how the potentiostat is connected to the samples to measure specific elements of the photocathode. CE is the counter electrode, RE is the reference electrode, and WE is the working electrode. (a) Light $J$ - $E$ measurement setup for spatioselectively catalyst-functionalized microwire photocathodes. (b) Dark $J-E$ measurement setup for determining the electrocatalytic behaviour of the samples. For light measurements the sample is placed at 1 sun intensity (see Section 5.3.8 for calibration details). The CE is a Pt mesh in a circle around the sample, in order not to intervene with the light. The RE is a $\mathrm{Ag} / \mathrm{AgCl}$ electrode, which is placed in close proximity of the sample. The WE is connected at the backside of the sample for light $J-E$ measurements and to the front for dark $J-E$ measurements, to bypass the silicon p-n junction. 


\begin{tabular}{lrrrrr} 
Route & Deposition time (s) & $V_{\mathrm{oc}}(\mathrm{V})$ & $J_{\mathrm{sc}}\left(\mathrm{mA} \mathrm{cm}^{-2}\right)$ & $F F(\%)$ & $\eta(\%)$ \\
\hline A & 0 & 0.46 & 37.6 & 65 & 11.1 \\
A & 10 & 0.46 & 35.5 & 64 & 10.3 \\
A & 15 & 0.46 & 32.8 & 64 & 9.7 \\
A & 30 & 0.46 & 29.4 & 60 & 8.0 \\
A & 180 & 0.44 & 4.2 & 63 & 1.1 \\
\hline B & 0 & 0.53 & 41.7 & 60 & 13.0 \\
B & 180 & 0.52 & 28.3 & 65 & 9.6
\end{tabular}

Table 5.1: $J-V$ characteristics for the Si micropillar arrays with radial $\mathrm{n}^{+} / \mathrm{p}$-junctions, without or with $\mathrm{Ni}-\mathrm{Mo}$ as catalyst, as determined from the $J-V$ graphs shown in Figures 5.6 and 5.7.

removal of the $\mathrm{SiO}_{2}$ from the microwire tops only ('Bare' in Figure 5.7) showed an increase in $V_{\text {oc }}$ as compared with non-passivated, fully exposed samples ('Bare' in Figure 5.6). Furthermore, the $J_{\mathrm{sc}}$ values were substantially higher, resulting in an increased efficiency of $13.0 \%$ (see Table 5.1) for the Si microwire arrays with $\mathrm{SiO}_{2}$.

As expected, high mass loading of electrodeposited Ni-Mo (180 s) affected only the $J_{\text {sc }}$ (measurement set-up shown in Figure 5.5), owing to blocking of light absorption (see Figure 5.7). A Ni-Mo deposition time of $180 \mathrm{~s}$ yielded $J_{\mathrm{sc}}=28.3 \mathrm{~mA} \mathrm{~cm}^{-2}$ for B samples as opposed to 4.2 $\mathrm{mA} \mathrm{cm}^{-2}$ for $A$ samples under the same deposition conditions. A longer deposition time gave a higher catalyst loading on the microwires, yet the decrease in $J_{\mathrm{sc}}$ was substantially lower than for A samples.

\subsubsection{Photoelectrochemical measurements}

Photoelectrochemical $(J-E)$ measurements under illumination were performed in $0.1 \mathrm{M}$ aqueous $\mathrm{H}_{2} \mathrm{SO}_{4}$ for fully exposed $\mathrm{Si}$ microwire arrays with and without a complete coating of Ni-Mo catalyst (A samples) and for top-exposed samples with spatioselective catalyst deposition (B) (Figures 5.10 and 5.11). The set-up is schematically represented in 


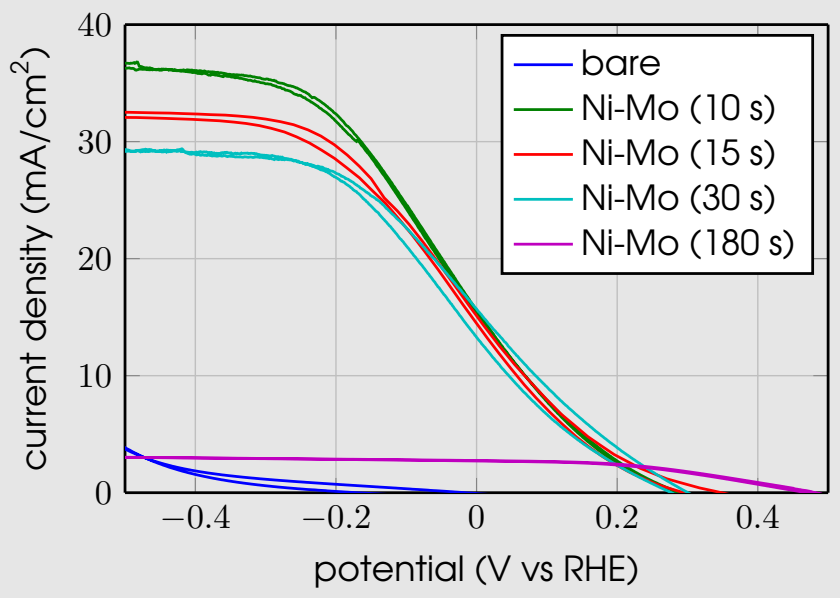

Figure 5.10: Current density $J_{\mathrm{ph}}$ versus potential for Si microwire array devices with radial $\mathrm{n}^{+} / \mathrm{p}$-junctions measured in cyclic potential sweeps, with and without electrodeposited Ni-Mo, using the deposition times shown, on fully exposed Si microwires. Data measured using AM $1.5 \mathrm{G}$ illumination and collected in $0.1 \mathrm{M} \mathrm{H}_{2} \mathrm{SO}_{4}$, referenced to the RHE potential.

Figure 5.9, in which the lamp is always positioned perpendicular to the substrate, to have minimal reflection or refraction through the different materials.

All of the relevant values ( $V_{\mathrm{oc}}, J_{\mathrm{ph}, \mathrm{RHE}}, F F$, plateau current density and $\eta_{\mathrm{IRC}}$ ) are tabulated in Table 5.2. Ideally, a well-performing photocathode has the same characteristics as the underlying PV cell $\left(J_{\mathrm{ph}, \mathrm{RHE}}\right.$ or $J_{\mathrm{sc}}$ respectively, $V_{\mathrm{oc}}$ and $F F$ ). Therefore, ideal regenerative cells (IRCs) are fully analogous to the solid-state PV cells described and measured above, and the efficiency ( $\eta_{\text {IRC }}$ ) of these systems is described by a similar equation as used to describe the efficiency of a PV device, (see equation 5.1) ${ }^{99}$.

$$
\eta_{\mathrm{IRC}}=\frac{V_{\mathrm{oc}} J_{\mathrm{ph}, \mathrm{RHE}} F F}{P_{\mathrm{in}}}
$$

The values of $V_{\mathrm{oc}}, F F$ and $J_{\mathrm{ph}, \mathrm{RHE}}$ (that is, the current density at $0 \mathrm{~V}$ versus RHE) in equation 5.1 are referenced to the equilibrium potential of 


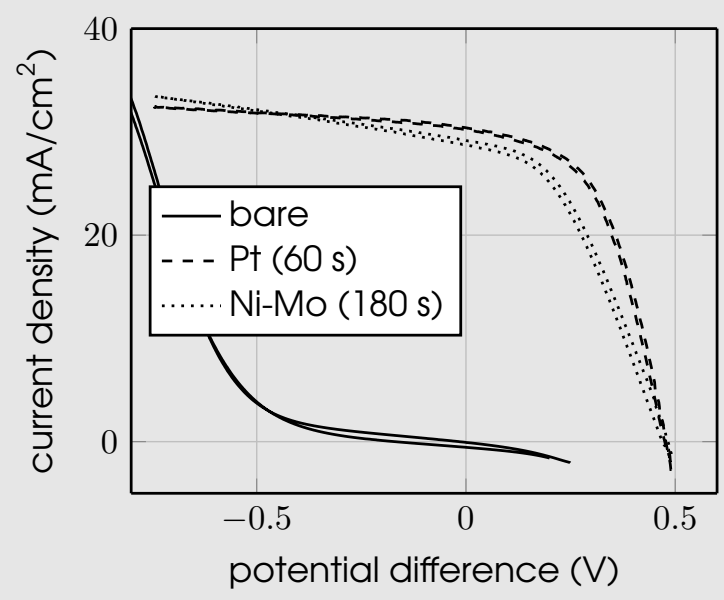

Figure 5.11: Current density $J_{\mathrm{ph}}$ versus potential for Si microwire array devices with radial $\mathrm{n}^{+} / \mathrm{p}$-junctions measured in cyclic potential sweeps, with and without Pt or Ni-Mo electrodeposited, using the deposition times shown, spatioselectively on top-exposed arrays. Data measured using AM 1.5G illumination and collected in $0.1 \mathrm{M} \mathrm{H}_{2} \mathrm{SO}_{4}$, referenced to the RHE potential.

the half-reaction being performed at the photocathode, which equals $\mathrm{RHE}$, and $P_{\text {in }}$ is the light power input (AM $1.5 \mathrm{G}, 100 \mathrm{~mW} \mathrm{~cm}^{-2}$ ).

Bare Si microwire arrays only produced $\mathrm{H}_{2}$ at a large overpotential (see Figures 5.10 and 5.11) which exemplifies once more the need for an HER catalyst. For sample type A, the incorporation of the Ni-Mo catalyst with a deposition time as short as 10 s enhanced the $\mathrm{H}_{2}$ production tremendously. This is witnessed by an increase in the photocurrent $\left(J_{\mathrm{ph}}\right)$ at $0 \mathrm{~V}$ versus RHE as compared with a bare sample. At a high overpotential, the performance of the underlying PV cell limits $J_{\mathrm{ph}}$ (that is, $J_{\mathrm{ph}}$ reaches a plateau), and the $J_{\mathrm{ph}}$ value matches well with the $J_{\mathrm{sc}}$ value of the same measured solid state PV cell (see Tables 5.1 and 5.2 for plateau current densities). The $F F$ increases substantially for a photocathode sample when coated with $\mathrm{Ni}-\mathrm{Mo}(\sim 12 \% F F)$, although the values are lower than the values reached for the solid-state PV cell $(\sim 65 \% F F)$. Only when the deposition time is increased to 180 s does the $F F$ increase to $45 \%$. Furthermore, the $V_{\text {oc }}$ of A samples with depo- 


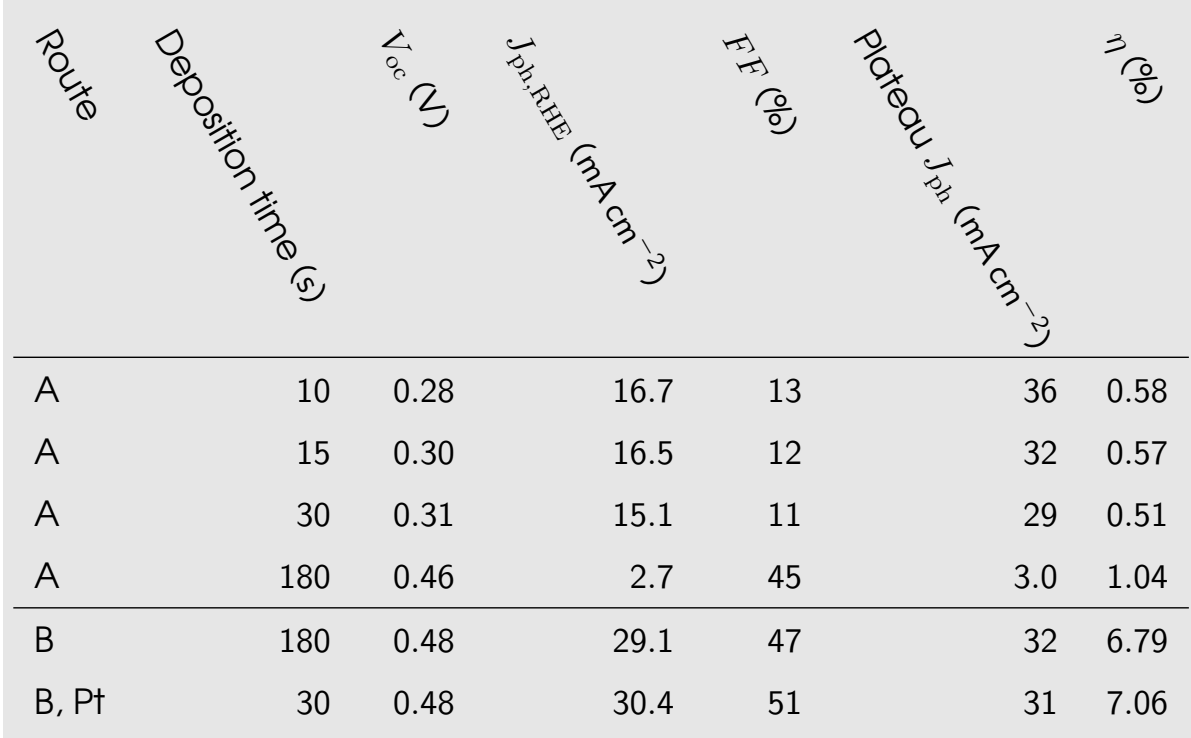

Table 5.2: $J-E$ characteristics for the Si micropillar arrays with radial $\mathrm{n}^{+} / \mathrm{p}$-junctions, with $\mathrm{Ni}-\mathrm{Mo}$ or Pt as catalyst, as determined from the $J-V$ graphs shown in Figures 5.10 and 5.11. The values of $V_{\mathrm{oc}}, J_{\mathrm{ph}, \mathrm{RHE}}$, $F F$ are referenced to the equilibrium potential of the half-reaction being performed at the photocathode, which equals RHE.

sition times up to $30 \mathrm{~s}$ is lower ( $\sim 0.30 \mathrm{~V}$ versus RHE) than that of the PV cell $(\sim 0.45 \mathrm{~V})$. The low $V_{\text {oc }}$ is probably due to a pinch-off effect, which has been described previously for Ni particles $(<50 \mathrm{~nm})$ on an n-type semiconductor ${ }^{116}$. Here we observed a similar particle size $(<50 \mathrm{~nm})$ on n-type Si by SEM (Figure 5.2). The $V_{\text {oc }}$ shifts to $0.42 \mathrm{~V}$ versus RHE when the deposition time is increased (180 s). Interestingly, even though $J_{\mathrm{ph}}$ is the lowest $\left(2.7 \mathrm{~mA} \mathrm{~cm}^{-2}\right)$ for the $180 \mathrm{~s}$ deposition time, $\eta_{\text {IRC }}(1.0 \%)$ is the highest of all samples of type A, owing to the high $F F$ value and increased $V_{\text {oc }}$.

Figure 5.11 presents the $J-E$ measurements of the microwire arrays with and without the spatioselectively deposited catalysts Ni-Mo and Pt (B samples), recorded under illumination. Table 5.2 contains the compiled parameters of Figure 5.11. Again, the Si microwire array with spatioselectively etched $\mathrm{SiO}_{2}$ without a catalyst exhibits a negligible 
$J_{\mathrm{ph}}$ at a potential of $0 \mathrm{~V}$ versus RHE. In contrast, deposition of either $\mathrm{Ni}-\mathrm{Mo}$ or $\mathrm{Pt}$ at the top of the microwire arrays leads to a substantial increase of $J_{\mathrm{ph}}$ at $0 \mathrm{~V}$ versus RHE. Furthermore, the $V_{\text {oc }}$ values for samples with locally deposited catalysts are substantially higher $(\sim 0.49 \mathrm{~V}$ versus RHE) than those observed for A samples with the catalyst deposited along the complete microwire length ( $\sim 0.30 \mathrm{~V}$ versus RHE). This is attributed to replacement of most of the silicon-electrolyte interface by a passivation layer. The values for $V_{\mathrm{oc}}, F F$ and $J_{\mathrm{ph}, \mathrm{RHE}}$ are in good agreement with the data for solid state PV cells (see Tables 5.1 and 5.2). Also note that we stay within the reductive limit, as in Figure 5.11 the cyclic voltammetry (CV) scan returns to $\sim 0 \mathrm{~mA} \mathrm{~cm}^{-2}$ for the Ni-Mo samples.

For comparison, Pt was also spatioselectively electrodeposited on the top-exposed microwires. Although it is a rare and precious metal in contrast to $\mathrm{Ni}$ and $\mathrm{Mo}$, $\mathrm{Pt}$ is one of the best-performing HER catalysts, as is already observed in the dark $J$ - $E$ measurements (Figure 5.8). The $\eta_{\text {IRC }}$ for B samples with a Pt catalyst is $7.1 \%$. The use of spatioselectively deposited Ni-Mo as a catalyst enhances the photoelectrochemical output tremendously as compared with A samples. The increase is mainly reflected in the $F F$ of the curve, which increases from $12 \%$ to $47 \%$. The best-performing microwire array with Ni-Mo has a $V_{\text {oc }}$ of $0.48 \mathrm{~V}, J_{\mathrm{ph}, \mathrm{RHE}}=29.1 \mathrm{~mA} \mathrm{~cm}^{-2}$ and a fill factor of $47 \%$, which results in $\eta_{\mathrm{IRC}}=6.8 \%$, which is highly comparable to the sample with Pt. The stability for $72 \mathrm{~h}$ and the Faradaic efficiency for HER of sample B have been tested as shown in Figures 5.12 and 5.13.

To gain more mechanistic insight into the parameters affecting the efficiency of the HER half-cell based on spatioselective coverage of Si microwires, we investigated the influences of the microwire pitch, in the range of 8 to $24 \mu \mathrm{m}$, and the spatioselective Ni-Mo coverage over these microwires, in the range of 2 to $36 \mu \mathrm{m}$ as measured from the top of the wires (see Figure 5.1(c)). These variations were made using the patterning method described in Section 3.2, because it turned out that the procedure in Figure 5.1(b), which uses a spin-coated polymer, had to be optimized separately for each geometry. Two exemplary HR-SEM images are given in Figure 5.14, with $19 \mu \mathrm{m}$ pitch and $18 \mu \mathrm{m}$ Ni-Mo coverage, and with $24 \mu \mathrm{m}$ pitch and $36 \mu \mathrm{m}$ Ni-Mo coverage, respectively, which show the great control over the deposition technique.

The wire coverage with Ni-Mo was varied at different pitches, and the catalyst activity was measured in the dark by contacting the emit- 


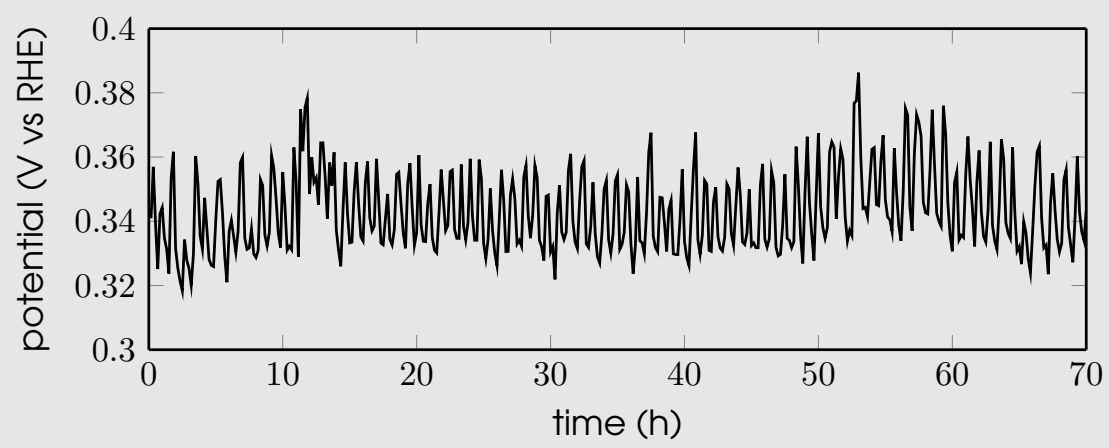

Figure 5.12: Recorded potential of a spatioselectively deposited NiMo catalyst on Si micropillars under continuous operation of $10 \mathrm{~mA} \mathrm{~cm}^{-2}$ and AM 1.5G simulated sunlight. Microwire array with $6 \mu \mathrm{m}$ pitch, upper $2 \mu \mathrm{m}$ functionalized with Ni-Mo (180 s deposition). The used setup to measure the activity is given in Figure 5.9(a). As noted, Ni-Mo does not exhibit infinite stability under strong acidic conditions ${ }^{117}$. To assess the durability of this catalyst we measured the potential vs RHE at a constant current density of $10 \mathrm{~mA} \mathrm{~cm}^{-2}$ in a buffered (pH 4.0) electrolyte of $0.2 \mathrm{M}$ potassium hydrogen phthalate for $72 \mathrm{~h}$ under $\mathrm{AM} 1.5 \mathrm{G}$ illumination. The average recorded potential appeared stable over the entire period of time, and the potential only oscillated, most probably due to hydrogen bubble formation and detachment.

ter $\left(\mathrm{n}^{+}\right.$-Si, schematic set-up given in Figure 5.9(b)) as the working electrode, in $0.1 \mathrm{M} \mathrm{H}_{2} \mathrm{SO}_{4}$ solution. The graphs of current density versus potential for all combinations (six different pitches and five different coverages) can be found in Figures 5.15 to 5.19. To summarize the effect of microwire pitch and coverage on the catalyst activity, current densities of all combinations are plotted at one potential, $-50 \mathrm{mV}$ versus RHE; see Figure 5.20 (black line in Figures 5.15 to 5.19). Clearly, to obtain optimal electrocatalytic activity, a trade-off has to be made between Ni-Mo coverage and microwire pitch. Smaller pitches are better, independent of covered wire length, but to overcome the limited activity of $\mathrm{Ni}$-Mo at larger pitches (lower right corner of Figure 5.20), a higher coverage of the microwire is required (upper right corner of Figure 5.20).

The fully finished microwire photocathodes with spatioselectively deposited catalyst were also measured as PV cells, without the electrolyte 


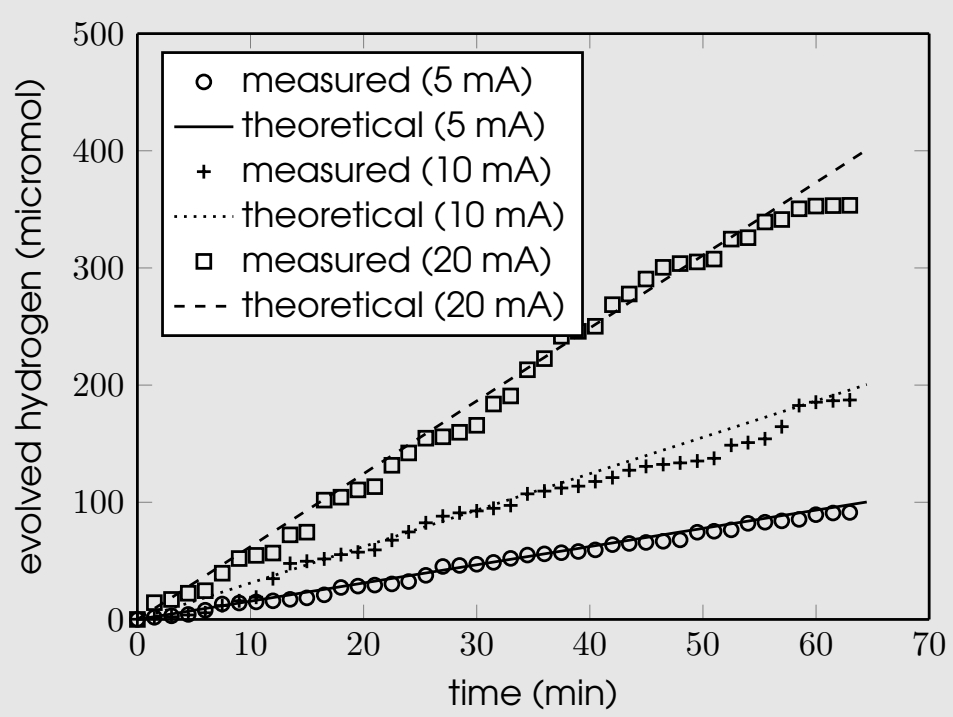

Figure 5.13: Calculated (lines) versus real (markers) hydrogen production using patterned Si microwire photocathode, biased at 5, 10 or $20 \mathrm{~mA}$. Microwire array with $6 \mu \mathrm{m}$ pitch, upper $2 \mu \mathrm{m}$ functionalized with $\mathrm{Ni}-\mathrm{Mo}$ (180 s deposition). The used setup to measure the activity is given in Figure 5.9(a). The $\mathrm{H}_{2}$ production for patterned Ni-Mo (180s) Si microwire samples B in Figure 5.11 was measured by gas chromatography (GC). Here, the $\mathrm{n}^{+} / \mathrm{p}$ junction was biased by contacting the emitter of the Si microwire photocathode and applying a bias to generate either 5,10 or $20 \mathrm{~mA}$ of current output. The calculated and measured produced $\mathrm{H}_{2}$ amounts match excellently, indicating a nearly $100 \%$ Faradaic efficiency.

(a schematic set-up is depicted in Figure 5.5(b)). The $J-V$ graphs of all combinations tested can be found in Figures 5.21 to 5.25. As with B samples, there was only a minor influence of the presence of Ni-Mo on $F F$ and $V_{\text {oc }}$. To assess the impact of the microwire coverage and pitch on the photovoltaic performance of the samples, in Figure 5.26 just the $J_{\mathrm{sc}}$ is shown in a contour plot, from which it becomes apparent that $J_{\mathrm{sc}}$ increases with increasing pitch and with decreasing degree of catalyst coverage (lower right corner of Figure 5.26). Conversely, the lower the microwire pitch and the higher the coverage, the lower is the current 

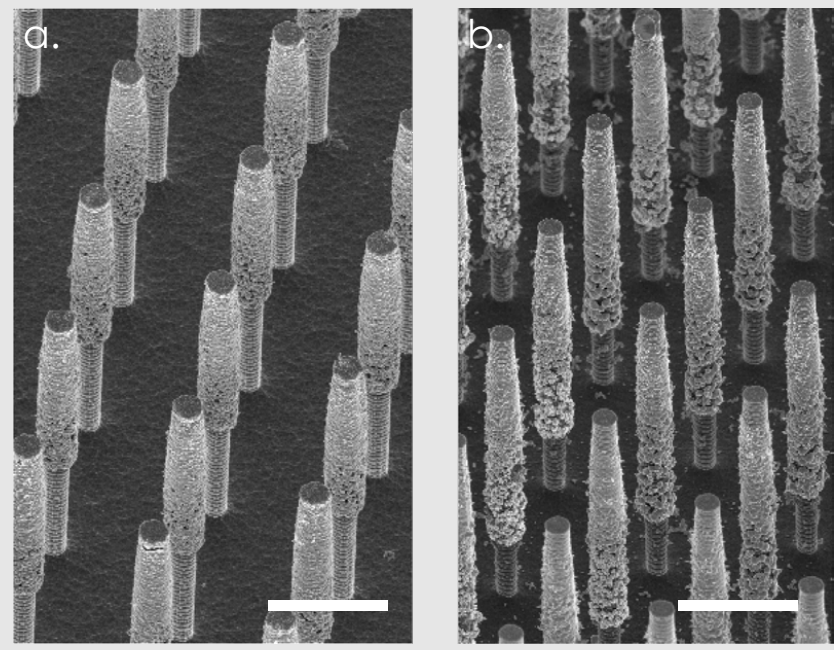

Figure 5.14: HR-SEM images of electrodeposition of earth-abundant catalyst (Ni-Mo) on Si microwire arrays with a radial junction. The microwires were passivated by $\mathrm{SiO}_{2}$ on the bottom part, while Ni-Mo was electrodeposited at the top. (a) Center-to-center distance between the microwires is $19 \mu \mathrm{m}$, and the top $18 \mu \mathrm{m}$ of the microwires is covered with catalyst. (b) Center-to-center distance between the microwires is $24 \mu \mathrm{m}$, and the top $36 \mu \mathrm{m}$ of the microwires is covered with catalyst. Scale bars are $15 \mu \mathrm{m}$.

density (upper left corner of Figure 5.26).

The contour plot Figure 5.27 of overall device efficiency $\eta_{\text {IRC }}$ versus pitch and wire coverage shows three domains (the $J-E$ graphs of all combinations are found in Figures 5.28 to 5.32 and were measured with the setup as in Figure 5.9(a)). In the lower right corner of Figure 5.27, the overall efficiency is dominated by the $F F$, which is a direct consequence of the limited amount of active catalyst (see also Figure 5.20). In detail, a photocathode behaves as the superposition of the catalyst behaviour in the dark (Figures 5.15 to 5.19) and the underlying PV cell (Figures 5.21 to 5.25). Because the resistances of the PV cells are found to be lower $\left(0.77-0.83 \Omega \mathrm{cm}^{2}\right)$ than the resistance imposed by the catalyst, we conclude that the $F F$ of a photocathode is mainly determined by the resistance that the catalyst imposes on the underlying 


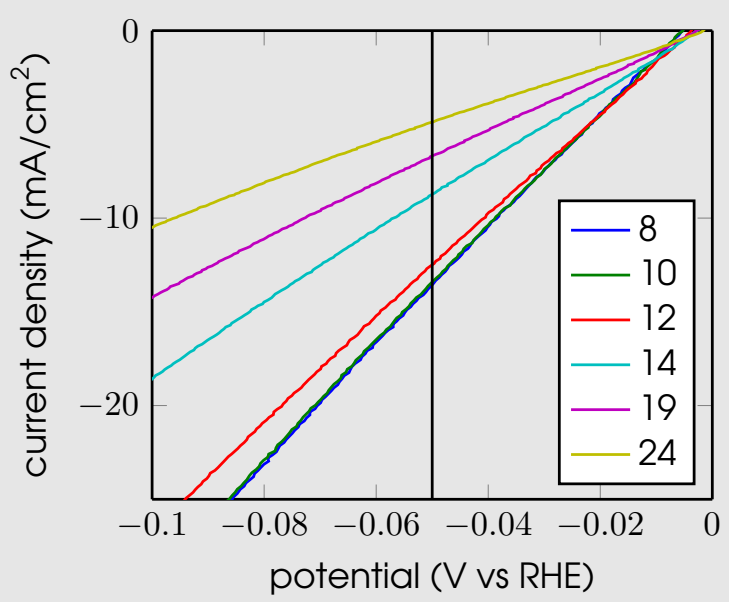

Figure 5.15: Plot of current density vs. potential, obtained for Si microwire arrays with various pitches as stated in the legend and the upper $2 \mu \mathrm{m}$ functionalized with Ni-Mo.

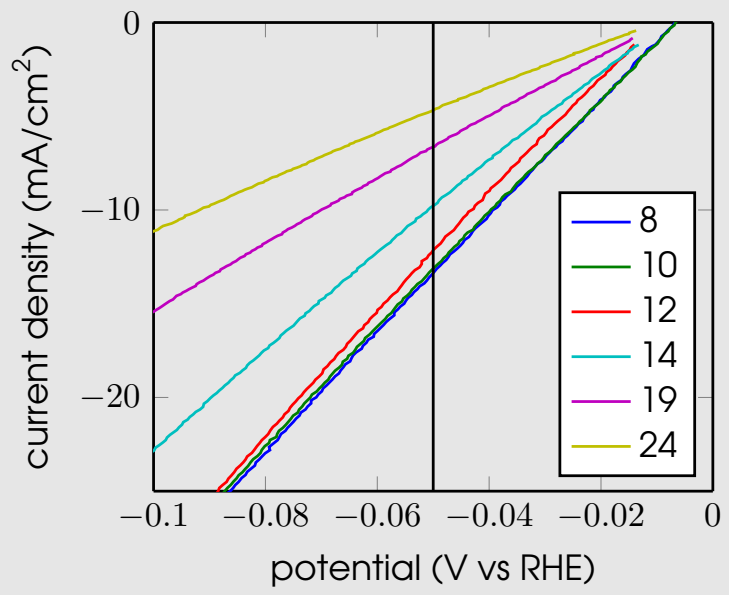

Figure 5.16: Plot of current density vs. potential, obtained for Si microwire arrays with various pitches as stated in the legend and the upper $9 \mu \mathrm{m}$ functionalized with Ni-Mo. 


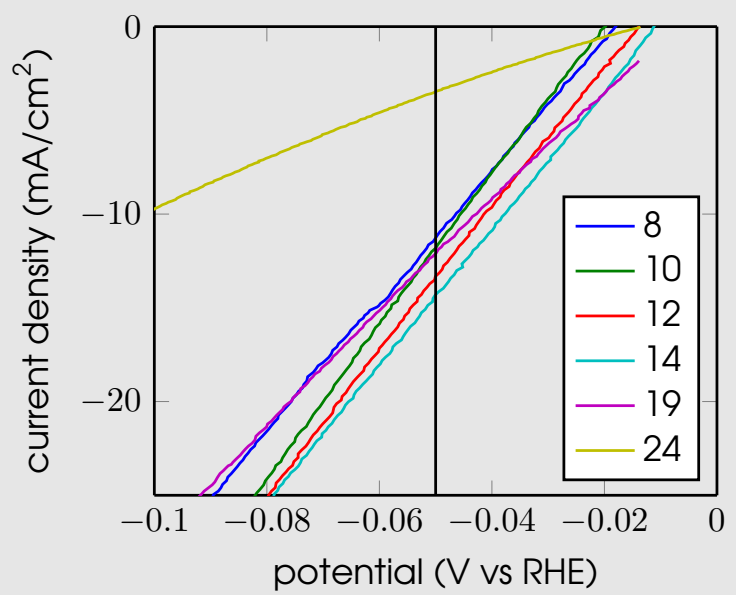

Figure 5.17: Plot of current density vs. potential, obtained for Si microwire arrays with various pitches as stated in the legend and the upper $18 \mu \mathrm{m}$ functionalized with Ni-Mo.

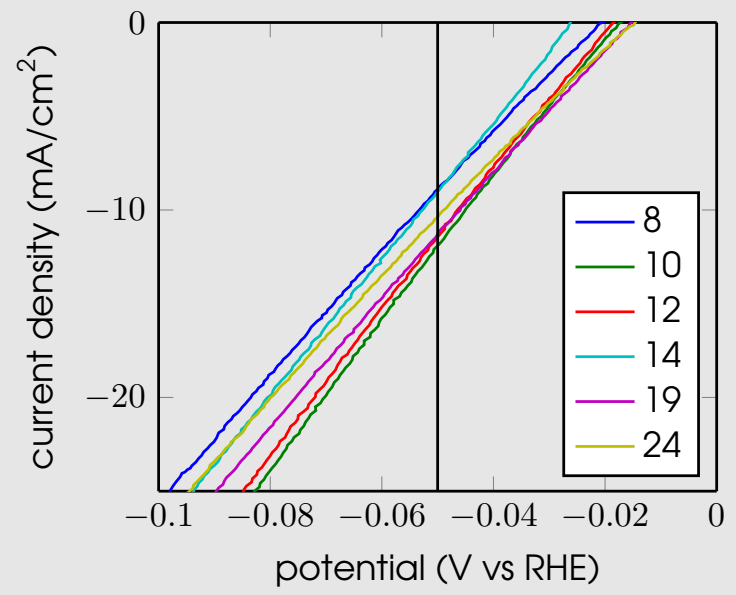

Figure 5.18: Plot of current density vs. potential, obtained for Si microwire arrays with various pitches as stated in the legend and the upper $25 \mu \mathrm{m}$ functionalized with Ni-Mo. 


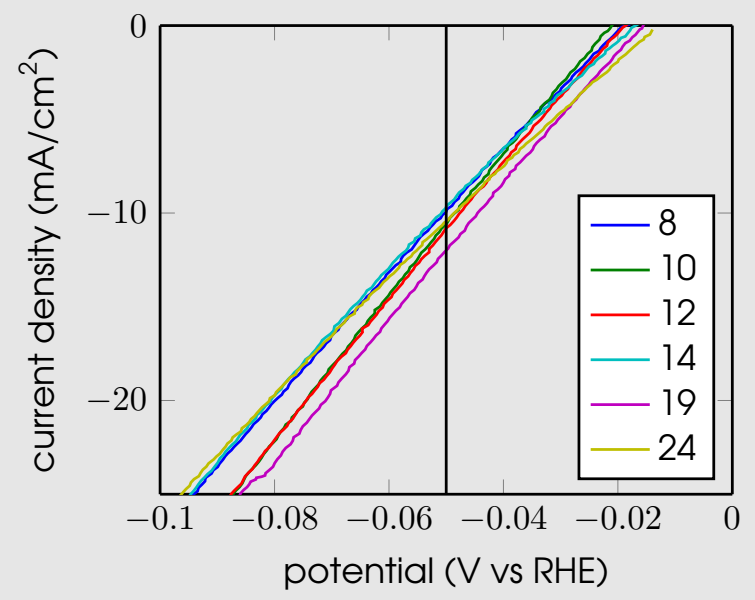

Figure 5.19: Plot of current density vs. potential, obtained for Si microwire arrays with various pitches as stated in the legend and the upper $36 \mu \mathrm{m}$ functionalized with Ni-Mo.

PV cell. There is an analytical approximation to calculate the $F F$ when the values of series resistance $\left(R_{\mathrm{s}}\right), V_{\mathrm{oc}}$ and short-circuit current $\left(I_{\mathrm{sc}}\right)$ are known ${ }^{118}$. The resistance $R_{\mathrm{s}}$ was here obtained for each variation by linearizing every data series in Figures 5.15 to 5.19 in the range between 0 and $50 \mathrm{mV}$. The calculated $\left(F F_{\mathrm{s}}\right)$ and measured $(F F)$ fill-factor values are tabulated in Tables 5.3 to 5.5. In all cases, the calculated values correspond well to the measured ones, thus supporting the idea that limitation by the catalyst is the main contributor to the fill factor. In the upper left corner of Figure 5.27, the overall efficiency is limited by light absorption. This is a direct consequence of reduced light absorption of the microwire array, because the catalyst blocks the incoming light, as is clear from Figure 5.26. Between these two zones, a region of optimal performance is found in which both the catalysis and the light absorption perform close to their maximum. In this zone, a pitch of $12 \mu \mathrm{m}$ and a spatioselective coverage of $2 \mu \mathrm{m}$ on the upper part of the $40 \mathrm{\mu m}$-long Si microwires results in the highest ideal regenerative cell efficiency (10.8\%).

To highlight the importance of catalysts on silicon microwires and nanowires in hydrogen-producing half-cells, we present an overview 


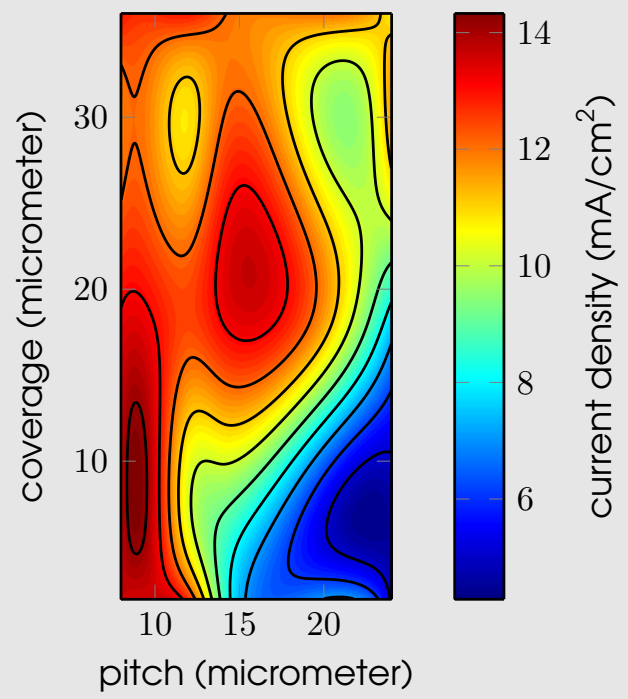

Figure 5.20: Contour plot of current density at $50 \mathrm{mV}$ versus RHE as a function of pitch and wire coverage, interpolated (cubic spline) from measurements on different devices.

of reported data of the best performing silicon wire-based photocathodes, subdivided into three categories: with $\mathrm{Ni}-\mathrm{Mo}$, with $\mathrm{Pt}$, or with any other catalyst in Table 5.6. To the best of our knowledge, our system outperforms previous half-cells constructed from either Si microwires or nanowires. A more complete list is given in Supplementary Table 4 of the paper version of this Chapter ${ }^{95}$. The efficiencies, if not given in the original literature reference, were calculated from the respective reported data, according to equation 5.1.

\subsection{Discussion}

We have designed, fabricated and experimentally validated a photoelectrode architecture that circumvents the trade-off between electrocatalytic activity of an opaque catalyst and optical absorption, parameters that determine $F F$ and $J_{\mathrm{ph}}$, respectively. By spatially and 


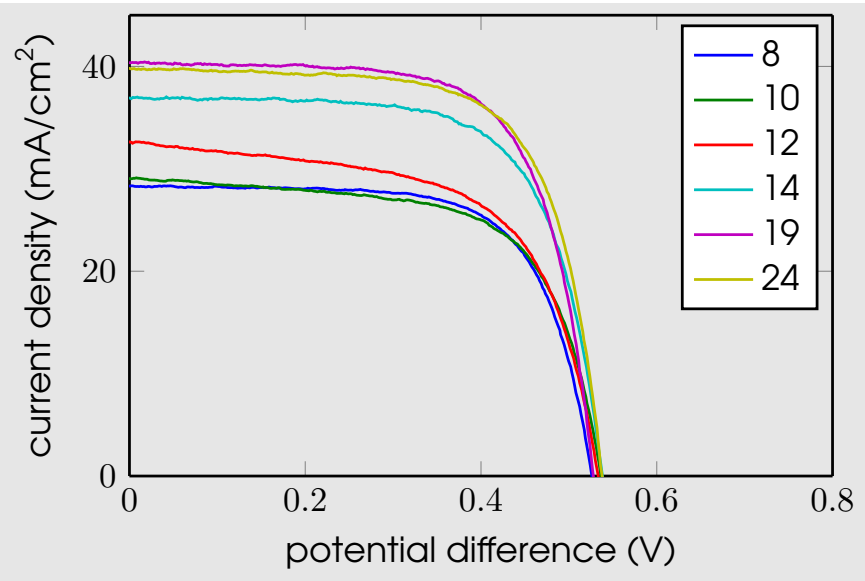

Figure 5.21: $J-V$ measurements of microwire substrates for different pitches as stated in the legends and the upper $2 \mu \mathrm{m}$ functionalized with $\mathrm{Ni}-\mathrm{Mo}$. Each line is the average of two different samples prepared with the same settings.

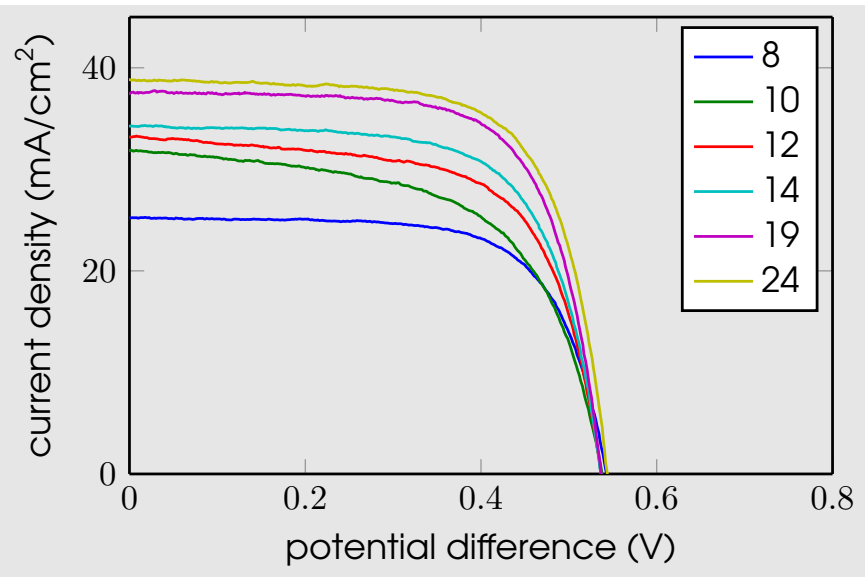

Figure 5.22: $J-V$ measurements of microwire substrates for different pitches as stated in the legends and the upper $9 \mu \mathrm{m}$ functionalized with $\mathrm{Ni}$-Mo. Each line is the average of two different samples prepared with the same settings. 


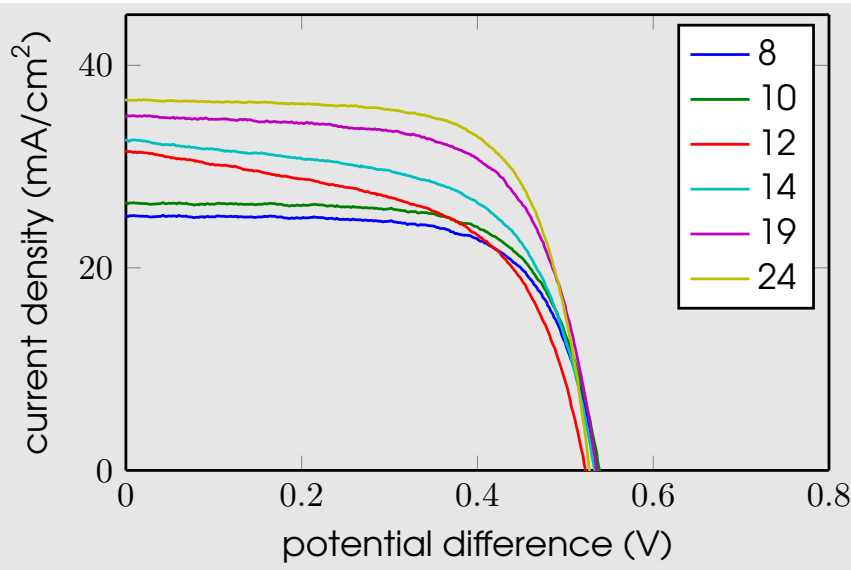

Figure 5.23: $J-V$ measurements of microwire substrates for different pitches as stated in the legends and the upper $18 \mu \mathrm{m}$ functionalized with Ni-Mo. Each line is the average of two different samples prepared with the same settings.

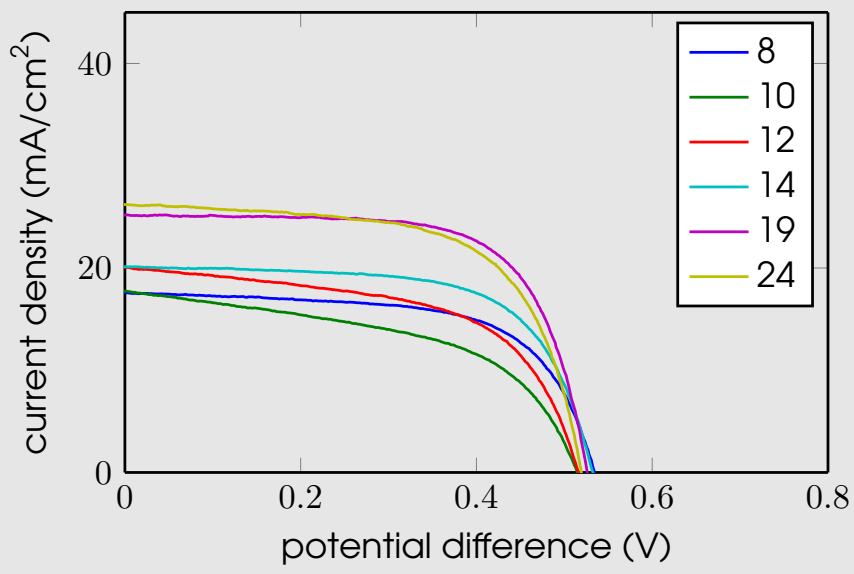

Figure 5.24: $J-V$ measurements of microwire substrates for different pitches as stated in the legends and the upper $25 \mu \mathrm{m}$ functionalized with Ni-Mo. Each line is the average of two different samples prepared with the same settings. 


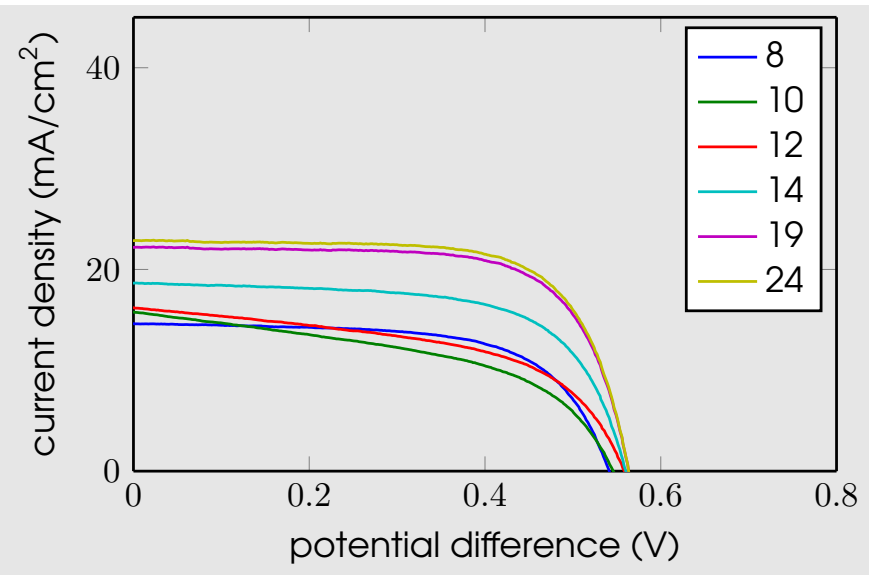

Figure 5.25: $J-V$ measurements of microwire substrates for different pitches as stated in the legends and the upper $36 \mu \mathrm{m}$ functionalized with Ni-Mo. Each line is the average of two different samples prepared with the same settings.

functionally decoupling light absorption and catalytic activity on highaspect-ratio silicon microwires with a radial $n^{+} / p$-junction, efficient solardriven hydrogen evolution has been achieved. This was first demonstrated and compared with an architecture which consisted of a high mass loading of Ni-Mo or Pt deposited spatioselectively on the upper $2 \mu \mathrm{m}$ of microwire arrays. This concept clearly demonstrates that precious metals (such as Pt) are not necessary to construct an efficient $\mathrm{H}_{2}$ photocathode. Two factors have been identified as limiting for the performance of photocathodes with opaque catalysts, insufficient catalyst loading $(F F)$ and parasitic light absorption $\left(J_{\mathrm{ph}}\right)$ by the catalyst. The first was found to dominate when the microwire arrays had a large pitch and only small parts of the wires were covered by catalyst. At a low pitch and high catalyst coverage, the performance was clearly limited by the light absorption performance of the underlying Si microwire photon absorber.

The photocathode configuration presented here has been functionally assessed upon front side illumination in an acidic electrolyte. The relevance of the spatioselective catalyst deposition method thus depends on an overall device configuration in which front side illumi- 


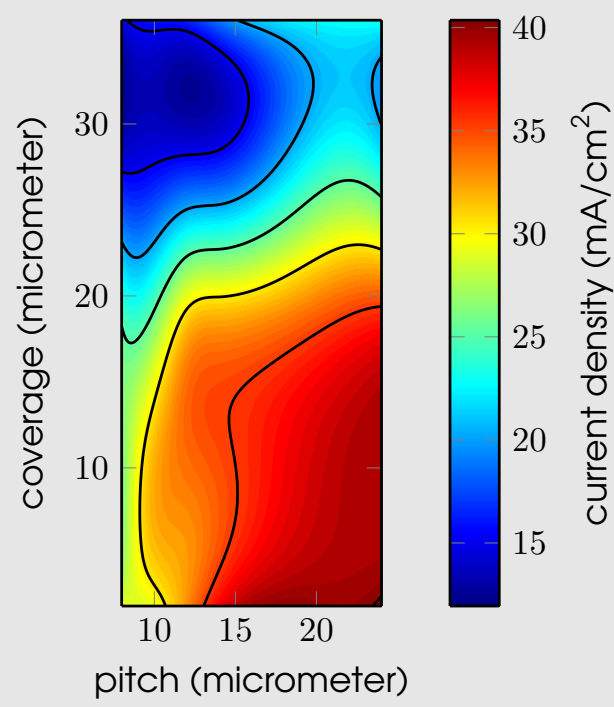

Figure 5.26: Contour plot of the current density $J_{\text {sc }}$ as a function of pitch and wire coverage, interpolated (cubic spline) from measurements on different devices.

nation is used. Therefore, we implemented our photocathode design in a full device using a wired device geometry. We have successfully demonstrated here such a full solar-to-fuel device architecture, and stable, unbiased water splitting has been shown over the course of an hour.

Our future research will focus on increasing the stability of the Si microwire photocathode in alkaline electrolytes and on increasing the produced photovoltage. The latter can be achieved, for example, by using a microwired tandem cell constructed of amorphous Si on top of crystalline Si microwires ${ }^{123}$. The silicon materials used in that study should be compatible with the fabrication process presented in the current study, possibly with adaptation of the patterning process parameters and/or the masking materials. Parasitic light absorption within the catalyst material should be prevented by spatioselective catalyst deposition, while maintaining high catalytic activity, as is demonstrated in the current study. We have demonstrated that, by careful design at the microscale, the chosen architecture provides high per- 


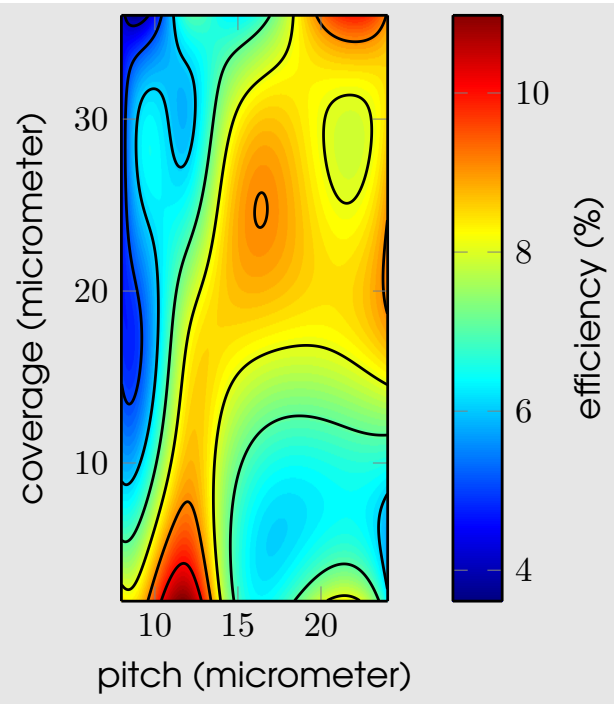

Figure 5.27: Contour plot of the overall device HER efficiency, $\eta_{\text {IRC }}$, as a function of pitch and wire coverage, interpolated (cubic spline) from measurements on different devices.

formance for an HER half-cell made entirely of earth-abundant materials. The concept should also be applicable to other material combinations, provided that fabrication methods can be found for those materials to achieve structuring at the right scale. The best-performing HER half-cell investigated here consisted of a Ni-Mo catalyst on the upper $2 \mu \mathrm{m}$ and a pitch of $12 \mu \mathrm{m}$, and demonstrated a $\eta_{\mathrm{IRC}}$ of $10.8 \%$.

\subsection{Methods}

\subsubsection{Fabrication of radial $n+/ p$ junctions in Si microwire arrays}

Figure 5.33 gives a schematic representation of the fabrication and passivation of an array of microwires with radial $\mathrm{n}^{+} / \mathrm{p}$ junctions. Substrates made of p-type Si (100), with a resistivity of $5.10 \Omega \mathrm{cm}, 100 \mathrm{~mm}$ diameter, $525 \mu \mathrm{m}$ thickness, single side polished (Okmetic Finland), were 


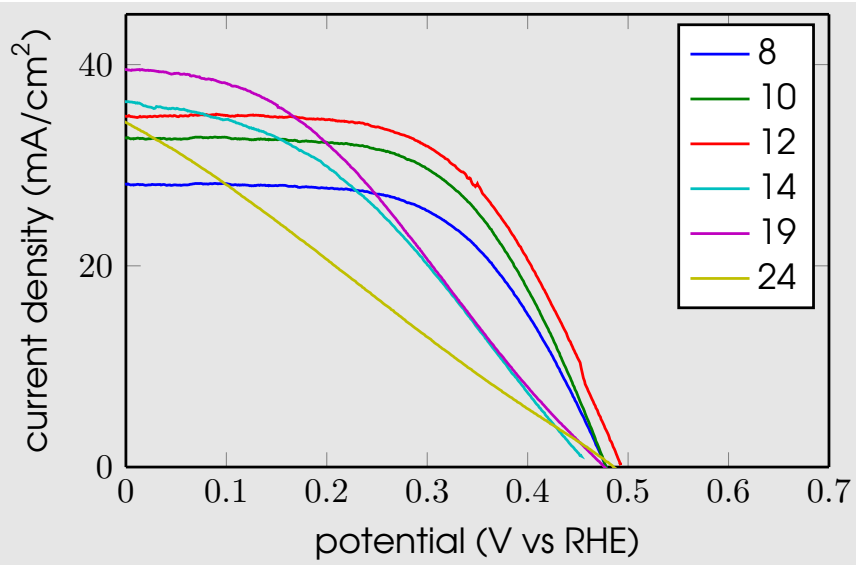

Figure 5.28: $J-E$ measurements of microwire substrates for different pitches as indicated and the upper $2 \mu \mathrm{m}$ functionalized with Ni-Mo. Each line is the average of two different samples prepared with the same settings.

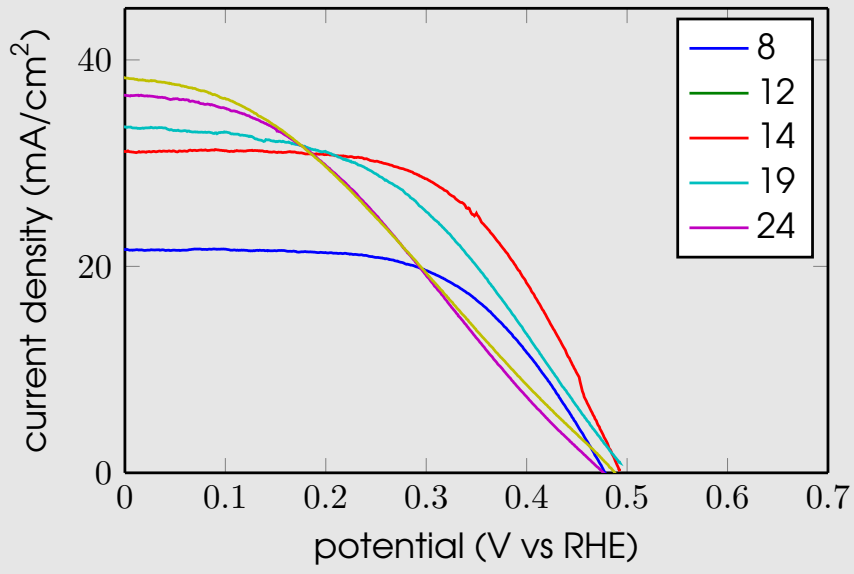

Figure 5.29: $J-E$ measurements of microwire substrates for different pitches as indicated and the upper $9 \mu \mathrm{m}$ functionalized with Ni-Mo. Each line is the average of two different samples prepared with the same settings. 


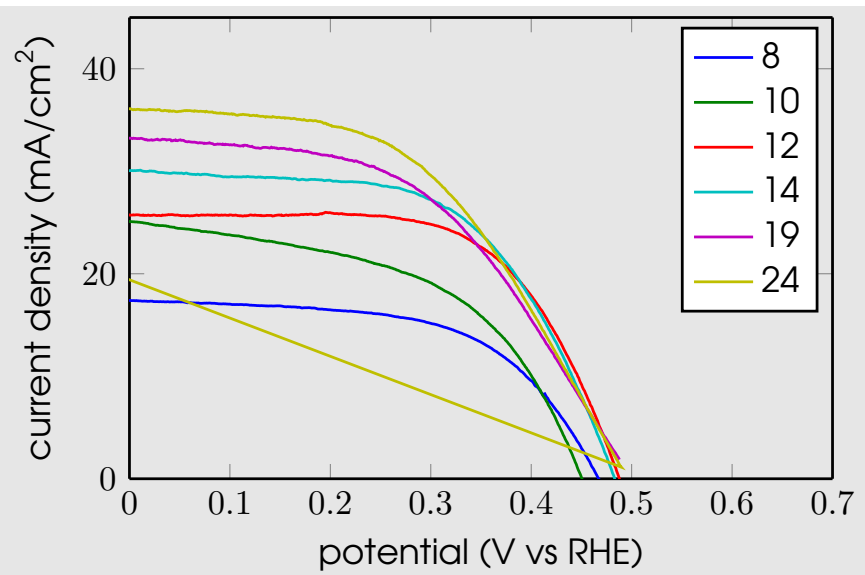

Figure 5.30: $J-E$ measurements of microwire substrates for different pitches as indicated and the upper $18 \mu \mathrm{m}$ functionalized with Ni-Mo. Each line is the average of two different samples prepared with the same settings.

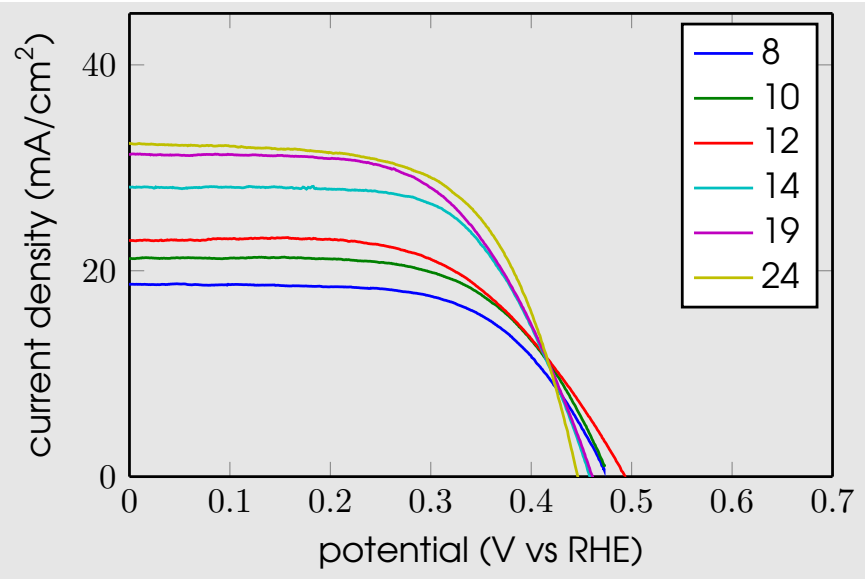

Figure 5.31: $J-E$ measurements of microwire substrates for different pitches as indicated and the upper $25 \mu \mathrm{m}$ functionalized with Ni-Mo. Each line is the average of two different samples prepared with the same settings. 


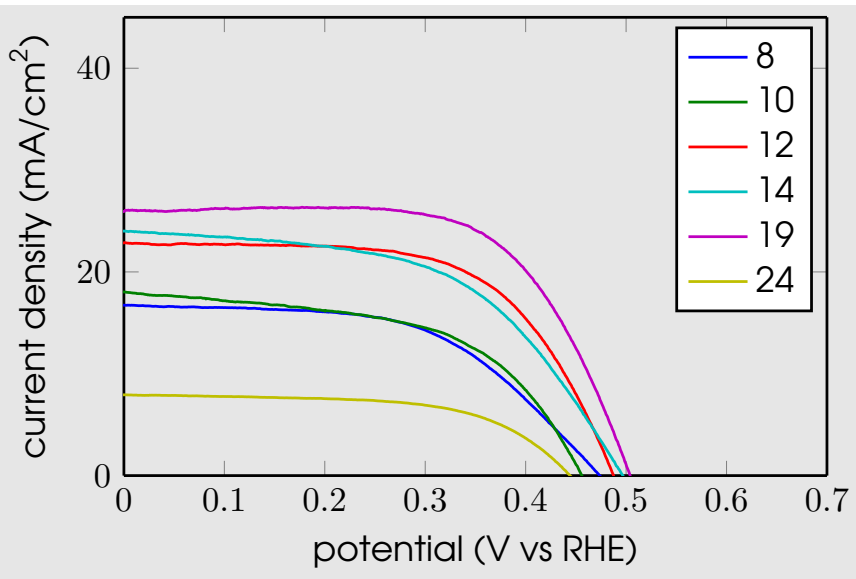

Figure 5.32: $J-E$ measurements of microwire substrates for different pitches as indicated and the upper $36 \mu \mathrm{m}$ functionalized with Ni-Mo. Each line is the average of two different samples prepared with the same settings.

prepared as previously reported ${ }^{6,103}$. In short, substrates were cleaned according to a standard procedure, and covered with $100 \mathrm{~nm}$ siliconrich silicon nitride (SiRN) by low-pressure chemical vapor deposition (LPCVD, $150 \mathrm{mTorr}, 77.5 \mathrm{SCCM}$ of $\mathrm{SiH}_{2} \mathrm{Cl}_{2}, 20 \mathrm{SCCM}$ of $\mathrm{NH}_{3}$ and $150 \mathrm{SCCM}$ of $\mathrm{N}_{2}$ at $850^{\circ} \mathrm{C}, 25 \mathrm{~min}$ ). The SiRN on the front side of the wafer was removed by reactive ion etching (RIE, with substrate temperature -10 ${ }^{\circ} \mathrm{C}, 20 \mathrm{SCCM} \mathrm{C} \mathrm{C}_{8}, 15 \mathrm{SCCM} \mathrm{CH}_{4}, 150 \mathrm{SCCM} \mathrm{He}$, pressure $8.5 \times 10^{-3} \mathrm{mbar}$, ICP power $2800 \mathrm{~W}$ and CCP power $350 \mathrm{~W}$ for $60 \mathrm{~s}$ ), and the substrate was cleaned by oxygen plasma (30 min; Tepla360 barrel etcher). By means of standard photolithography, squares $\left(5 \cdot 5 \mathrm{~mm}^{2}\right)$ with hexagonally packed circles ( $4 \mu \mathrm{m}$ diameter, and varying pitch from sample to sample between $6 \mu \mathrm{m}$ and $24 \mu \mathrm{m}$ ) were defined in a photoresist polymer (Olin 906-12). Silicon microwires (40 $\mu \mathrm{m}$ in height) were etched into the silicon substrate by deep reactive ion etching (DRIE, Bosch process; a cyclic process using $\mathrm{SF}_{6}$ for silicon etching and $\mathrm{C}_{4} \mathrm{~F}_{8}$ to create a passivation layer on the sidewalls) and the substrates were cleaned subsequently in oxygen plasma (30 min). The $\mathrm{n}^{+} / \mathrm{p}$ junctions were formed by the deposition of phosphorus oxide (LPCVD, $350 \mathrm{mTorr}, 675^{\circ} \mathrm{C}, 30 \mathrm{SCCM}$ $\mathrm{N}_{2}$ through the tetraethyl orthosilicate (TEOS) evaporator to form a 50 


\begin{tabular}{l|rrrrrr}
\hline pitch $(\mu \mathrm{m})$ & 8 & 10 & 12 & 14 & 19 & 24 \\
\hline & \multicolumn{5}{|c}{$2 \mu \mathrm{m}$ coverage } \\
\hline$V_{\mathrm{oc}}(\mathrm{V})$ & 0.48 & 0.48 & 0.49 & 0.49 & 0.48 & 0.49 \\
$\left.J_{\mathrm{sc}}(\mathrm{mA} \mathrm{cm})^{-2}\right)$ & 28.99 & 33.54 & 35.55 & 37.33 & 40.84 & 41.28 \\
$R_{\mathrm{s}}\left(\Omega \mathrm{cm}^{2}\right)$ & 3.67 & 3.71 & 3.98 & 5.73 & 7.43 & 10.26 \\
$F F(\%)$ & 60.69 & 59.33 & 62.32 & 43.18 & 37.67 & 35.86 \\
$F F_{\mathrm{s}}(\%)$ & 61.20 & 58.20 & 59.37 & 45.37 & 35.36 & 31.52 \\
\hline & \multicolumn{7}{|c}{$9 \mu \mathrm{m}$ coverage } \\
\hline$V_{\mathrm{oc}}(\mathrm{V})$ & 0.48 & 0.49 & 0.49 & 0.49 & 0.48 & 0.49 \\
$\left.J_{\mathrm{sc}}(\mathrm{mA} \mathrm{cm})^{-2}\right)$ & 21.63 & 25.44 & 31.11 & 33.52 & 36.57 & 38.26 \\
$R_{\mathrm{s}}\left(\Omega \mathrm{cm}^{2}\right)$ & 3.72 & 3.79 & 4.89 & 5.07 & 7.47 & 10.57 \\
$F F(\%)$ & 57.88 & 59.02 & 57.50 & 46.20 & 35.93 & 33.19 \\
$F F_{\mathrm{s}}(\%)$ & 60.87 & 60.06 & 59.73 & 46.39 & 36.33 & 36.50
\end{tabular}

Table 5.3: Cell characteristics from Figures 5.15 and 5.16 and Figures 5.21 and 5.22 for various pitches and catalyst coverages. $R_{\mathrm{s}}$ was obtained by linearizing the data series (resulting slope $=1 / R_{\mathrm{s}}$ ) of the catalytic activity of Figures 5.15 to 5.19 , between 0 and $50 \mathrm{mV}$ vs RHE. The calculated $F F_{\mathrm{s}}$ values were obtained using the analytical expression developed by Green ${ }^{118}$.

SCCM TEOS/ $\mathrm{N}_{2}$ mixture, $150 \mathrm{SCCM} \mathrm{O}_{2}, 16.5 \mathrm{SCCM} \mathrm{PH}$ and $313.5 \mathrm{SCCM}$ argon). The substrate was annealed at $1050^{\circ} \mathrm{C}$ for $15 \mathrm{~min}$ to accomplish diffusion of the dopant into the silicon microwire array to form the appropriate radial junction. Before the deposition of the passivation layer, the dopant oxide was stripped in BHF solution $(10 \mathrm{~min})$, upon which the wafers returned to their hydrophobic state (Figure 5.33(e)). Subsequently, the wafers were cleaned by immersion in fuming $99 \%$ nitric acid $\left(\mathrm{HNO}_{3}, 2\right.$ times $\left.5 \mathrm{~min}\right)$, boiling $69 \%$ nitric acid $(10 \mathrm{~min})$ and immersion in $1 \%$ aqueous hydrofluoric acid (HF) to remove the native oxide ( $1 \mathrm{~min}$ ). The $100 \mathrm{~nm}$ passivation layer of SISiO2 was applied by LPCVD and is described in more detail below. After selective removal of the 


\begin{tabular}{l|rrrrrr}
\hline pitch $(\mu \mathrm{m})$ & 8 & 10 & 12 & 14 & 19 & 24 \\
\hline & \multicolumn{5}{|c}{$18 \mu \mathrm{m}$ coverage } \\
\hline$V_{\mathrm{oc}}(\mathrm{V})$ & 0.47 & 0.45 & 0.49 & 0.48 & 0.49 & 0.49 \\
$\left.J_{\mathrm{sc}}(\mathrm{mA} \mathrm{cm})^{-2}\right)$ & 17.27 & 24.43 & 25.69 & 29.80 & 33.05 & 35.85 \\
$R_{\mathrm{s}}\left(\Omega \mathrm{cm}^{2}\right)$ & 4.50 & 4.16 & 3.75 & 2.78 & 4.14 & 4.42 \\
$F F(\%)$ & 58.25 & 52.54 & 63.29 & 58.69 & 50.52 & 50.68 \\
$F F_{\mathrm{s}}(\%)$ & 65.45 & 50.25 & 63.37 & 60.42 & 50.11 & 50.72 \\
\hline & \multicolumn{7}{|c}{$25 \mu \mathrm{m}$ coverage } \\
\hline$V_{\mathrm{oc}}(\mathrm{V})$ & 0.47 & 0.47 & 0.49 & 0.46 & 0.46 & 0.45 \\
$\left.J_{\mathrm{sc}}(\mathrm{mA} \mathrm{cm})^{-2}\right)$ & 18.71 & 21.23 & 22.96 & 28.00 & 31.23 & 32.17 \\
$R_{\mathrm{s}}\left(\Omega \mathrm{cm}^{2}\right)$ & 5.54 & 4.20 & 4.38 & 3.38 & 4.37 & 4.82 \\
$F F(\%)$ & 62.38 & 62.15 & 57.33 & 63.61 & 59.14 & 62.18 \\
$F F_{\mathrm{s}}(\%)$ & 61.27 & 63.73 & 62.92 & 62.00 & 59.05 & 60.87
\end{tabular}

Table 5.4: Cell characteristics from Figures 5.17 and 5.18 and Figures 5.23 and 5.24 for various pitches and catalyst coverages. $R_{\mathrm{s}}$ was obtained by linearizing the data series (resulting slope $=1 / R_{\mathrm{s}}$ ) of the catalytic activity of Figures 5.15 to 5.19 , between 0 and $50 \mathrm{mV}$ vs RHE. The calculated $F F_{\mathrm{s}}$ values were obtained using the analytical expression developed by Green ${ }^{118}$.

surface passivation coating from the tops of the microwires, a Ni-Mo alloy was deposited on the microwires by electrodeposition as described below. Finally, ohmic contacts $(1 \mu \mathrm{m}$ thick $\mathrm{Al} / \mathrm{Si}, 99 / 1 \%)$ were sputtered (Oxford PL400) on the front and back sides of the wafer.

\subsubsection{LPCVD of silicon dioxide passivation layer}

Conformal deposition of a silicon oxide $\left(\mathrm{SiO}_{2}\right)$ film was done by means of LPCVD. $\mathrm{SiO}_{2}$ was deposited at $725^{\circ} \mathrm{C}$ with a $50 \mathrm{SCCM}$ tetraethyl orthosilicate flow (200 mTorr, $8.1 \mathrm{~nm} \mathrm{~min}{ }^{-1}$ deposition rate). The total thick- 


\begin{tabular}{l|rrrrrr}
\hline pitch $(\mu \mathrm{m})$ & 8 & 10 & 12 & 14 & 19 & 24 \\
\hline \multicolumn{7}{|c}{$36 \mu \mathrm{m}$ coverage } \\
\hline$V_{\mathrm{oc}}(\mathrm{V})$ & 0.47 & 0.46 & 0.49 & 0.50 & 0.50 & 0.44 \\
$J_{\mathrm{sc}}\left(\mathrm{mA} \mathrm{cm}{ }^{-2}\right)$ & 16.59 & 17.62 & 22.71 & 23.72 & 25.98 & 7.87 \\
$R_{\mathrm{s}}\left(\Omega \mathrm{cm}^{2}\right)$ & 5.02 & 4.70 & 4.62 & 4.96 & 4.20 & 4.75 \\
$F F(\%)$ & 54.66 & 55.62 & 61.75 & 53.96 & 64.91 & 60.67 \\
$F F_{\mathrm{s}}(\%)$ & 62.01 & 60.99 & 58.50 & 56.63 & 58.71 & 60.22
\end{tabular}

Table 5.5: Cell characteristics from Figures 5.19 and 5.25 for various pitches and catalyst coverages. $R_{\mathrm{s}}$ was obtained by linearizing the data series (resulting slope $=1 / R_{\mathrm{s}}$ ) of the catalytic activity of Figures 5.15 to 5.19 , between 0 and $50 \mathrm{mV}$ vs RHE. The calculated $F F_{\mathrm{s}}$ values were obtained using the analytical expression developed by Green ${ }^{118}$.

ness of $\mathrm{SiO}_{2}$ was set to $100 \mathrm{~nm}$.

\subsubsection{Local removal of silicon dioxide from the tops of $\mathrm{Si}$ microwire arrays}

To selectively remove $\mathrm{SiO}_{2}$ from the tops of the microwires, the following protocol was applied. HMDS (hexamethyldisilazane) was spincoated between the wires (4000 rpm, $2 \mathrm{~min}$ ), and subsequently AZ9260 photoresist was spin-coated (1000 rpm, $4 \mathrm{~min}$ ). The samples were dried overnight at $10 \times 10^{-3} \mathrm{mbar}$ at room temperature. The photoresist was then removed around the microwire arrays by masking the arrays with squares of $5 \cdot 5 \mathrm{~mm}^{2}$. The samples were exposed to light ( 3 times $10 \mathrm{~s}$ ultraviolet exposure, $10 \mathrm{~s}$ delay between exposure cycles) and developed $(7 \mathrm{~min})$. The remaining photoresist within the microwire arrays was exposed to light with a blank mask (3 times 10 s ultraviolet exposure, $10 \mathrm{~s}$ delay between exposure cycles). The resist layer between the microwires was reduced in thickness using RIE for $\sim 3 \mathrm{~min}\left(100 \mathrm{SCCM} \mathrm{O}_{2}\right.$, $100 \mathrm{mTorr}, 25 \mathrm{~W}, 10^{\circ} \mathrm{C}$ ), until the desired height of photoresist around the microwires was obtained (Figure 5.33(d); see Figure 5.34 for HR-SEM im- 


\begin{tabular}{l|lllll} 
& & & & & \\
\end{tabular}

Table 5.6: Compilation of microwire-based and nanowire-based hydrogen-producing half-cells. Measurements done under AM 1.5G simulated sunlight in their best-performing electrolyte. $\mathrm{MW}=$ microwire, NW = nanowire. 


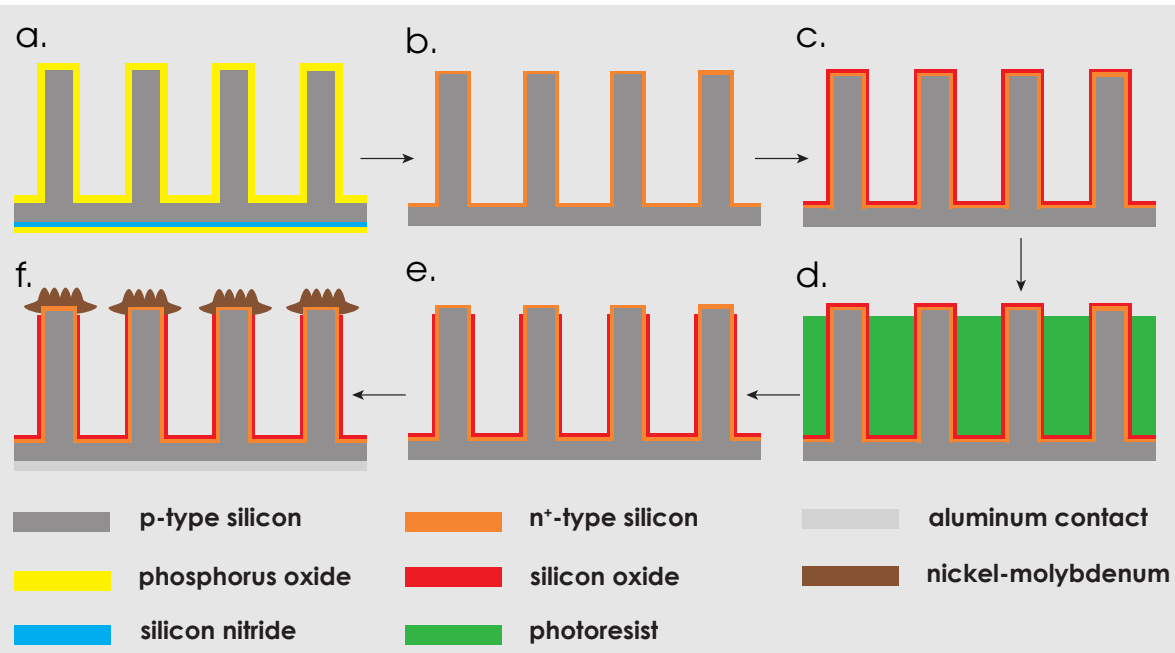

Figure 5.33: Schematic fabrication process: (a) Wire array obtained after deep reactive ion etching of a silicon wafer with a photoresist pattern on the front side and silicon-rich silicon nitride on the backside, followed by the deposition of the dopant oxide by LPCVD (phosphorus). (b) Formation of the radial junction, by a drive-in step at $1050^{\circ} \mathrm{C}$ for 15 min. (c) Deposition of a passivation layer of $\mathrm{SiO}_{2}$ by LPCVD. (d) Filling of the wire arrays with photoresist, followed by controlled retraction by $\mathrm{O}_{2}$ plasma. (e) Selective removal of the passivation layer by BHF wet etching. (f) Deposition of aluminum back contacts by sputtering and of the catalyst by electrodeposition.

ages). $\mathrm{SiO}_{2}$ was selectively removed from the exposed tops by wet etching in BHF ( $\sim 1 \mathrm{~min}$, etch rate $\left.210 \mathrm{~nm} \mathrm{~min}^{-1}\right)$. The remaining photoresist layer was stripped by 10 min ultrasonication in acetone, followed by $10 \mathrm{~min}$ ultrasonication in isopropanol and spin-drying. The selectively etched microwire arrays showed clearly exposed tops (Figure 5.34).

\subsubsection{Electrical contacts}

To ensure ohmic contact to the solar cells, $1 \mu \mathrm{m}$ aluminum/silicon alloy (99\% Al, $1 \% \mathrm{Si}$ ) was sputtered (Oxford PL 400) on the front and back 

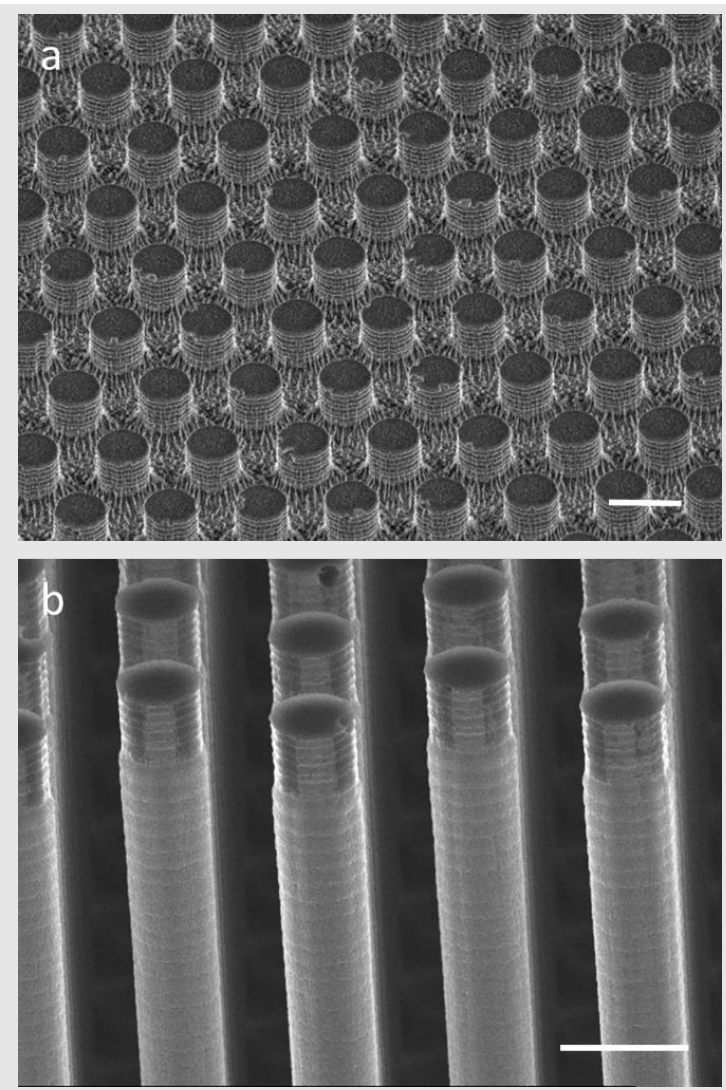

Figure 5.34: (a) HR-SEM images of Si microwire arrays with photoresist between the microwires, which is etched by $\mathrm{O}_{2}$ plasma. (b) $\mathrm{Si}$ microwire array with selectively removed $\mathrm{SiO}_{2}$ from the tops by etching (a) in BHF. Scale bars are $5 \mu \mathrm{m}$ 
sides of each specimen, where on the front side a shadow mask was applied to protect the wire arrays from coating. In this way, a square electrode was formed around the wire array. Directly before coating, all native silicon oxide was stripped from the silicon surface by etching for $1 \mathrm{~min}$ in $1 \mathrm{wt} \%$ aqueous HF solution.

\subsubsection{Catalyst deposition}

$\mathrm{Ni}-\mathrm{Mo}$ was deposited by electrodeposition directly after forming the ohmic contacts as described above, to limit formation of a new oxide layer on the silicon as much as possible. The Ni-Mo catalyst was deposited in a two-electrode set-up, by solely contacting the $\mathrm{n}^{+}$-Si emitter through the aluminum contact on the front. The counter-electrode was a Pt mesh. The electrodeposition bath consisted of an aqueous solution of $1.3 \mathrm{M}$ nickel(II) sulphate hexahydrate, $0.5 \mathrm{M}$ boric acid, and $25 \mathrm{mM}$ sodium molybdate in Milli- $Q$ water. The samples were covered with a ring so that only a circular area of $6 \mathrm{~mm}$ diameter was exposed to the electrolyte. Electrodeposition of Ni-Mo catalyst on the Si microwires was performed at a constant current of $5.5 \mathrm{~mA}\left(20 \mathrm{~mA} \mathrm{~cm}^{-2}\right.$ with respect to projected sample area not covered by the ring) for time periods described in Table 5.7.

Platinum was deposited from an aqueous solution of $5 \mathrm{mM}$ hexachloroplatinic acid $\left(\mathrm{H}_{2} \mathrm{PtCl} 6\right)$ and $0.5 \mathrm{M}$ sodium sulfate $\left(\mathrm{Na}_{2} \mathrm{SO}_{4}\right)$, potentiostatically at $-0.7 \mathrm{~V}$ vs Ag/AgCl. Charge densities of $130 \mu \mathrm{Cm}^{-2}$ were supplied.

\subsubsection{J-V measurements}

To measure the electrical characteristics of the coated Si microwires, samples were positioned perpendicular to a light source (see Figure 5.5). $J-V$ measurements were recorded on a VersaSTAT 4 potentiostat using a linear voltage sweep from -0.7 to $0.7 \mathrm{~V}$ at a rate of $0.2 \mathrm{~V} \mathrm{~s}^{-1}$. The light intensity was calibrated to AM 1.5G illumination at the position of the sample, using the calibrated light source described below. 


\begin{tabular}{r|r|rrrrr} 
& Coverage $(\mu \mathrm{m})$ & 2 & 9 & 18 & 22 & 35 \\
\hline Pitch $(\mu \mathrm{m})$ & $f_{\mathrm{c}}(\%)$ & \multicolumn{5}{c}{$t_{\mathrm{D}}(\mathrm{s})$} \\
\hline 8 & 22.7 & 180 & 270 & 540 & 630 & 1080 \\
10 & 14.5 & 65 & 195 & 390 & 455 & 780 \\
12 & 10.1 & 45 & 135 & 270 & 315 & 540 \\
14 & 7.4 & 33 & 99 & 198 & 231 & 396 \\
19 & 4.0 & 18 & 54 & 108 & 126 & 216 \\
24 & 2.5 & 12 & 36 & 72 & 84 & 144
\end{tabular}

Table 5.7: Deposition time of Ni-Mo catalyst on microwire arrays for every microwire pitch/coverage combination. Here, $f_{\mathrm{c}}$ is the filling fraction, in order to give a quick comparison with the results of Chen et al. ${ }^{102}$. The geometric filling fraction in the study of Chen et al. ${ }^{102}$ is defined as the ratio of the geometric area of the catalyst to the total geometric area of the photoelectrode. Therefore, $f_{\mathrm{c}}$ is directly related to the pitch of our Si microwires when only the tops of the microwires with a spatioselective catalyst are taken into account. $t_{\mathrm{D}}$ is the deposition time for every chosen pitch and coverage height. The HER catalytic activity of Ni-Mo was essentially unchanged when the nominal deposition time was $>180 \mathrm{~s}$ for microwire arrays with a pitch of $8 \mu \mathrm{m}$ and the top $2 \mu \mathrm{m}$ covered with $\mathrm{Ni}-\mathrm{Mo}$, as is seen from Figure 5.8. The deposition time for each sample in this study was corrected for absolute surface area of exposed silicon. In that way it was tried to achieve approximately the same catalyst layer thickness in all cases.

\subsubsection{J-E measurements}

The coated microwire photocathodes were photoelectrochemically tested using a potentiostat (VersaSTAT 4) in a three electrode configuration, in which the microwire photocathode with an exposed projected surface area of $0.28 \mathrm{~cm}^{2}$ acted as the working electrode (WE), a platinum wire mesh as the counter-electrode (CE) and an $\mathrm{Ag} / \mathrm{AgCl}$ electrode as the reference electrode (RE). The electrolyte used was $0.1 \mathrm{M}$ aqueous sulfuric acid $\left(\mathrm{H}_{2} \mathrm{SO}_{4}\right), \mathrm{pH}$. A set-up as shown in Figure 5.9(b) was used for dark $J-E$ measurement; the $\mathrm{n}^{+}-\mathrm{Si}$ emitter was 


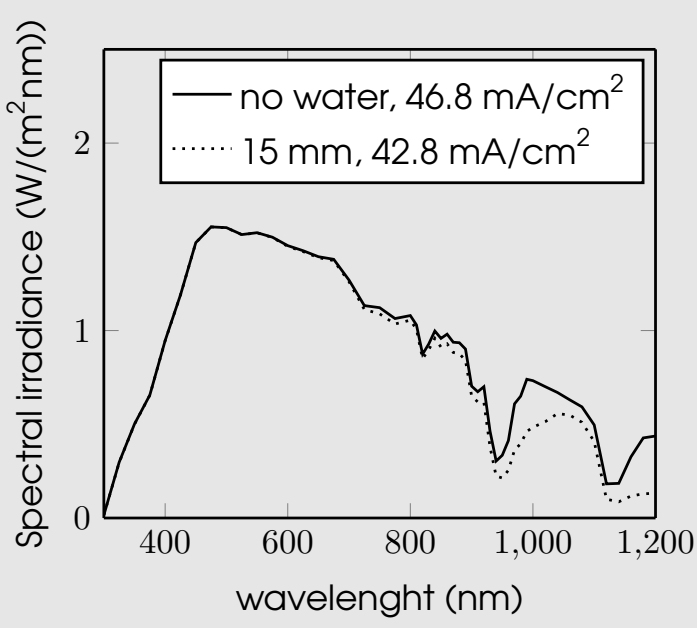

Figure 5.35: Plot of the ASTM reference pattern (solid) and calculated transmittance through $15 \mathrm{~mm}$ of water (dotted). Each graph was integrated in the range of 300 to $1200 \mathrm{~nm}$ to calculate the maximum photocurrent.

contacted as the WE, directly through the aluminum contact on the front. For each sample, five full cyclic voltammetry measurements were done at a scan rate of $10 \mathrm{mV} \mathrm{s}^{-1}$.

For $J$-E measurements under illumination, the backside $\mathrm{p}$-Si base was contacted through the aluminum contact on the back. Five full cyclic voltammetry measurements were done at a scan rate of 10 $\mathrm{mV} \mathrm{s}^{-1}$. Samples were positioned perpendicular to a solar spectrum light source as described below. The configuration is shown in Figure 5.9(a). The light intensity was calibrated to AM 1.5G illumination at the position of the sample. Here, continuous scans were recorded instead of chopped light.

The water layer on top of the sample was $15 \mathrm{~mm}$ thick. The absolute light absorption by the solution would lead to a decrease of $3 \mathrm{mAcm}^{-2}$ if it is assumed that every photon leads to an electron. The absorption was calculated from the Lambert-Beer relation and integrated over the solar spectrum in the range of $300-1200 \mathrm{~nm}$ (see Figure 5.35) ${ }^{124}$. 


\subsubsection{Light source and calibration}

The light source that was used is a $300 \mathrm{~W}$ xenon arc light source, fitted with an Air Mass filter (AM 1.5G) from Newport, Oriel Instruments. Upon installation, the lamp was calibrated by Newport. Before every measurement, the lamp was checked by a calibrated reference solar cell (91150V). The $91150 \mathrm{~V}$ reference cell and meter consist of a readout device, a $2 \cdot 2 \mathrm{~cm}^{2}$ calibrated solar cell made of monocrystalline silicon and a KG5 window. The cell is equipped with a thermocouple assembled in accordance with IEC 60904-2. The certification is accredited by the National Institute of Standards and Technology (NIST) to the ISO-17025 standard. It reads solar simulator irradiance in Sun units, whereby one Sun is equal to $1000 \mathrm{~W} \mathrm{~m}^{-2}$ at $25^{\circ} \mathrm{C}$ and AM 1.5 Global Reference spectrum.

The solar simulator was checked for spectral mismatch by a spectrometer (AvaSpec-ULS2048XL-EVO) fitted with a CC-VIS/NIR, slit size $10 \mu \mathrm{m}, 1.4 \mathrm{~nm}$ resolution in the range $300-1050 \mathrm{~nm}$, an integration time of $8 \mathrm{~ms}$ and averaged over 60 scans. The spectral scan and an ASTM reference pattern are given in Figure 5.36. The current density (assuming $100 \%$ incident photon-to-current efficiency, IPCE) of the calibrated lamp and the ASTM spectrum match very well, $40.1 \mathrm{~mA} \mathrm{~cm}^{-2}$ and $40.8 \mathrm{~mA} \mathrm{~cm}^{-2}$ respectively, in the range of $300-1050 \mathrm{~nm}$.

\subsubsection{Gas chromatography}

The reactor was connected to a gas chromatograph (Compact GC, Interscience), equipped with a Parabond $Q$ column $(10 \mathrm{~m})$ and a thermal conductivity detector to determine the amount of $\mathrm{H}_{2}$ in the argon carrier gas. $5 \mathrm{ml} \mathrm{min}^{-1}$ argon was flowed through the electrolyte and sampled every 90 s for the concentration of $\mathrm{H}_{2}$. 


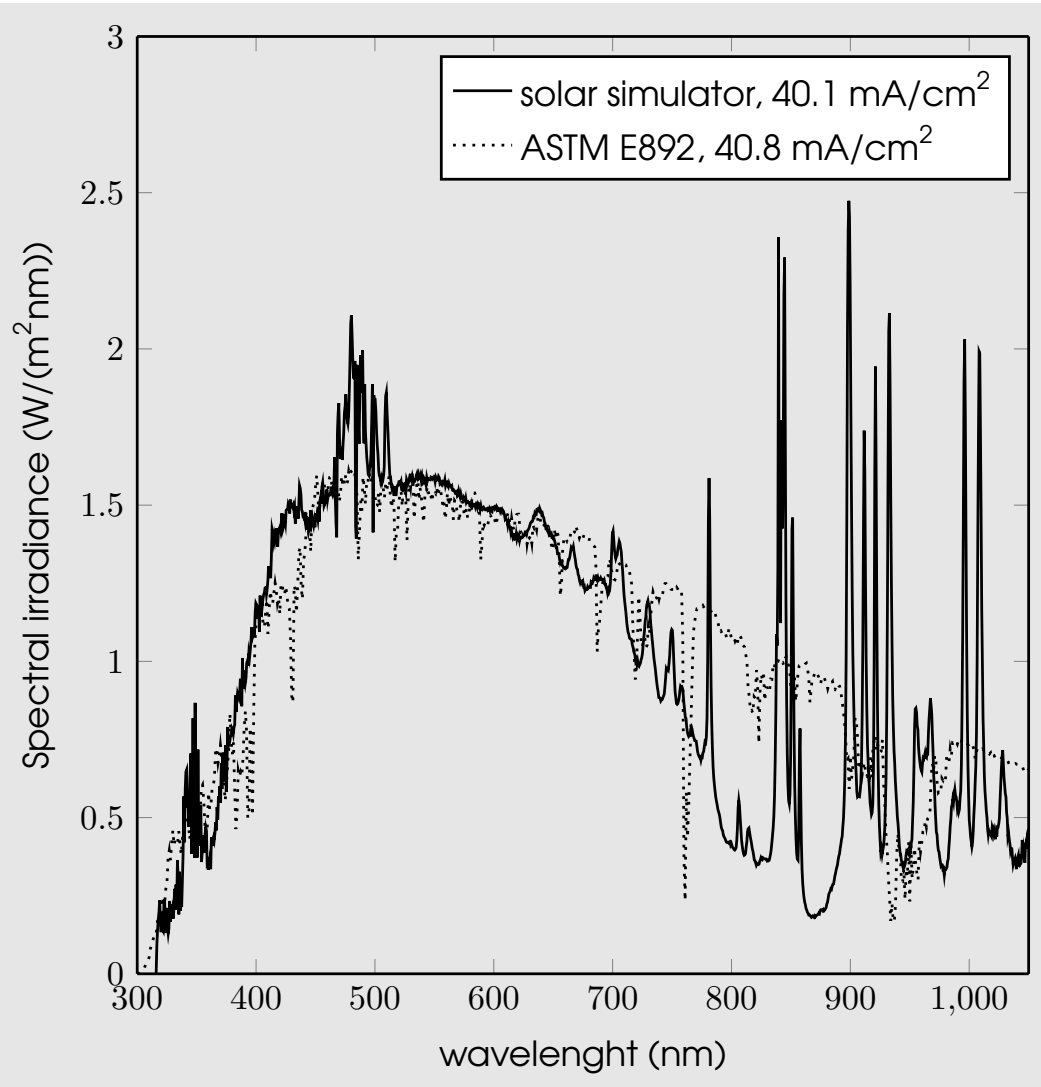

Figure 5.36: Spectral irradiance plot of an ASTM reference pattern (dotted) and of the solar simulator used for the measurements in this thesis (solid). 


\section{Chapter 6}

\section{A photoanode: hematite-silicon tandem}

As discussed in Section 2. 1.2, a tandem of two semiconductor materials, one with a band gap around $1.0 \mathrm{eV}$ and one with a band gap around 1.6-1.8 eV, would theoretically be the most efficient way of converting light from the sun to a current for electrochemical water splitting. Silicon, the most commonly used light absorber ${ }^{100}$ has a band gap of $1.1 \mathrm{eV}$ and in state-of-the-art devices performs close to the theoretical maximum efficiency of this material, and can be used as the low band gap light absorber. For higher band gap semiconductors made of earth-abundant elements, anatase $\mathrm{TiO}_{2}, \mathrm{WO}_{3}, \mathrm{BiVO}_{4}, \alpha-\mathrm{Fe}_{2} \mathrm{O}_{3}$ and $\mathrm{Ta}_{3} \mathrm{~N}_{5}$ are the most commonly studied photoelectrode materials. Of these, the latter two have the lowest band gap (around $2.1 \mathrm{eV}^{125}$ ), and therefore theoretically have the highest potential for forming an efficient tandem with silicon. Since hematite $\left(\alpha-\mathrm{Fe}_{2} \mathrm{O}_{3}\right)$ is stable under alkaline conditions, for which there are also earth-abundant catalyst materials available ${ }^{14}$, possibilities for making an integrated tandem of this material with silicon are investigated in this Chapter.

Holes generated upon illumination of hematite will typically not diffuse more than a few nanometers. Therefore only very thin layers can be used ${ }^{125}$. However, if the thin layer is on a flat surface, most of the sunlight will pass the layer without being absorbed. Therefore nano or microstructuring approaches are necessary to increase the photocur- 
rents produced by this material ${ }^{125}$. The nanostructure achieved by the synthesis routes described in this Chapter, as well as the extent to which these synthesis methods can coat a microstructured substrate, are of special concern in the discussion of these synthesis routes.

\subsection{Hematite synthesis routes}

Several synthesis routes for hematite were explored in the course of this research. The best results for each route are presented here, together with the necessary fabrication details.

\subsubsection{Electrophoretic deposition}

In electrophoretic deposition, particles in suspension are charged and forced towards a substrate with the use of an electric field. Several authors have recently used this method for the synthesis of porous hematite layers for use as a photoanode ${ }^{126-129}$, where Zong et al. ${ }^{126}$ achieved a photocurrent of $\sim 1.1 \mathrm{~mA} \mathrm{~cm}^{-2}$ under 1 sun AM $1.5 \mathrm{G}$ illumination. In all these reports, iron oxide nanoparticles were synthesized hydrothermally, washed subsequently with water and acetone, and while still wet were dispersed in acetone. The particles were charged by dissolving iodine in the acetone, and then electrophoretic deposition was done by applying $10 \mathrm{~V}$ for $30-240 \mathrm{~s}$ between two parallel submerged electrodes $0.8-1 \mathrm{~cm}$ apart. Finally the layers were annealed at $700-800{ }^{\circ} \mathrm{C}$ in air.

Unfortunately we were unable to measure any photocurrent from samples that we tried to prepare in this way. Therefore a different synthesis route was tried for synthesizing nanoparticles. A solution of $1 \mathrm{~g}$ of $\mathrm{Fe}\left(\mathrm{NO}_{3}\right)_{3} \cdot 9 \mathrm{H}_{2} \mathrm{O}$ and $0.3 \mathrm{~g}$ of polyvinylpyrrolidone in $100 \mathrm{ml} \mathrm{DI}$ water was prepared. Under stirring and ultrasonication $65 \mathrm{ml}$ of $0.1 \mathrm{M} \mathrm{NaOH}$ solution (in DI water) was added dropwise to create a finely dispersed $\mathrm{FeOOH}$ precipitate at a $\mathrm{pH}$ of $\sim 12$. The suspension was left until the precipitate had settled to the bottom half of the liquid and the top half was replaced by water, and the precipitate was redispersed by stirring. This was repeated 3 times with water, and then three times with acetone. $17 \mathrm{ml}$ from this suspension was taken and added to $93 \mathrm{ml}$ of 
acetone. This suspension was stirred for three hours and then sonicated for half an hour. Another $88 \mathrm{ml}$ of acetone and $22 \mathrm{ml}$ of acetylacetone (the necessity of using the latter was not tested) was added and the suspension was stirred overnight and sonicated for half an hour. $0.09 \mathrm{~g}$ of iodine was added, and after stirring for another hour the electrophoretic deposition was performed. Two $2 \cdot 2 \mathrm{~cm}^{2}$ samples of oxidized silicon on which $\mathrm{a} \sim 100 \mathrm{~nm}$ thick layer of $\mathrm{F}: \mathrm{SnO}_{2}$ (FTO) was sputtered were submerged up to $\sim 1.7 \mathrm{~cm}$ into the suspension with the conductive surfaces facing each other. $10 \mathrm{~V}$ was applied between the two electrodes for $1 \mathrm{~min}$, after which the sample was removed quickly from the solution before switching off the power supply, and the deposited layer was left to dry in air horizontally. The layers were annealed for $20 \mathrm{~min}$ at $800^{\circ} \mathrm{C}$ in air (nitrogen atmosphere was also tried but resulted in no measurable photocurrent).

As can be seen from the SEM pictures (Figure 6.1), a rather flat, 300$900 \mathrm{~nm}$ thick layer was formed, with some cracks. The physical contact with the conductive FTO layer seems poor. The sample was clamped to a $6 \mathrm{~mm}$ diameter opening on the side of a $10 \mathrm{ml}$ cell, with a glass window on the opposite side and a platinum mesh (outside the light path) as a counter electrode, and a $\mathrm{Hg} / \mathrm{HgO}$ reference electrode, which was filled with $1 \mathrm{M} \mathrm{NaOH}$ solution in Milli- $Q$ water. The sample was illuminated with a 1 sun AM $1.5 \mathrm{G}$ solar spectrum in the same way as described in Section 5.3.7. Cyclic voltammograms were recorded $\left(50 \mathrm{mV} \mathrm{s}^{-1}\right)$ in the light and dark, and the fifth scan is reported for each situation in Figure 6.2. A photocurrent of almost $0.1 \mathrm{~mA} \mathrm{~cm}^{-2}$ at $1.23 \mathrm{~V}$ vs RHE was observed. The reduction peak around $0.8 \mathrm{~V}$ vs RHE in the voltammogram recorded under illumination, as well as the reasonable catalytic activity can perhaps be explained by impurities that may have been present in the electrolyte or the layer.

It was shown that this synthesis method can easily produce photoanode layers. None of the synthesis parameters was optimized and it is not unlikely that the photocurrent can be increased by further optimization. Furthermore the use of a structured substrate, a co-catalyst, surface passivation, doping and a heterojunction have all been reported to be able to increase the performance of hematite ${ }^{125}$, and they may have a positive effect on the performance of electrophoretically deposited hematite photoanodes. 

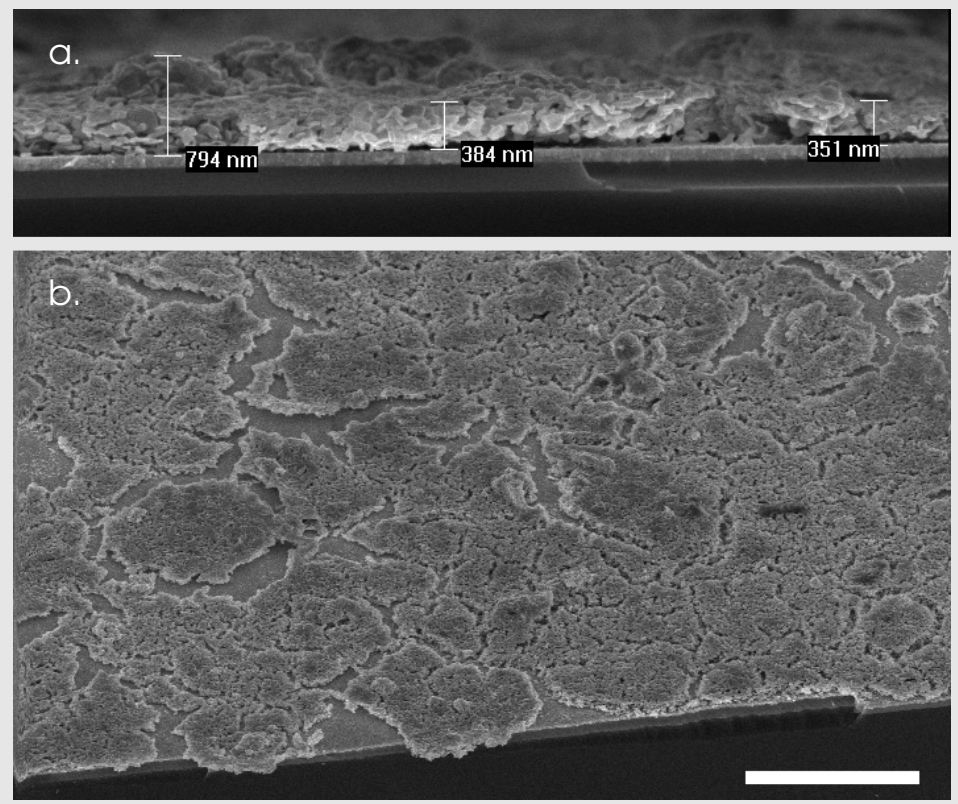

Figure 6.1: SEM pictures of a hematite layer prepared by electrophoretic deposition of iron hydroxide particles from a suspension acetone, after annealing: (a) cross section, (b) overview, scale bar is $5 \mu \mathrm{m}$.

\subsubsection{Hydrothermal growth}

Some of the best-performing hematite photoanodes recently reported in literature are based on the facile and scalable synthesis method of in-situ hydrothermal growth $125,130,131$, where Gurudayal et al. ${ }^{131}$ achieved a photocurrent of $3.12 \mathrm{~mA} \mathrm{~cm}^{-2}$ under 1 sun AM $1.5 \mathrm{G}$ illumination. In this procedure, conductive substrates are put in a high ionic strength, somewhat acidic aqueous solution containing $\mathrm{Fe}^{3+}$ ions inside an autoclave and heated at $100^{\circ} \mathrm{C}$ for several hours, resulting in a uniform nanostructured layer of $\mathrm{FeOOH}$. This is transformed to hematite by annealing at $550-800{ }^{\circ} \mathrm{C}$ in air.

Several hydrothermal synthesis recipes were tried. The best results were achieved with a method based on the report of Vayssieres et al. ${ }^{132}$. The $\mathrm{FeOOH}$ layer was grown by heating a $15 \mathrm{ml}$ aqueous solution with 


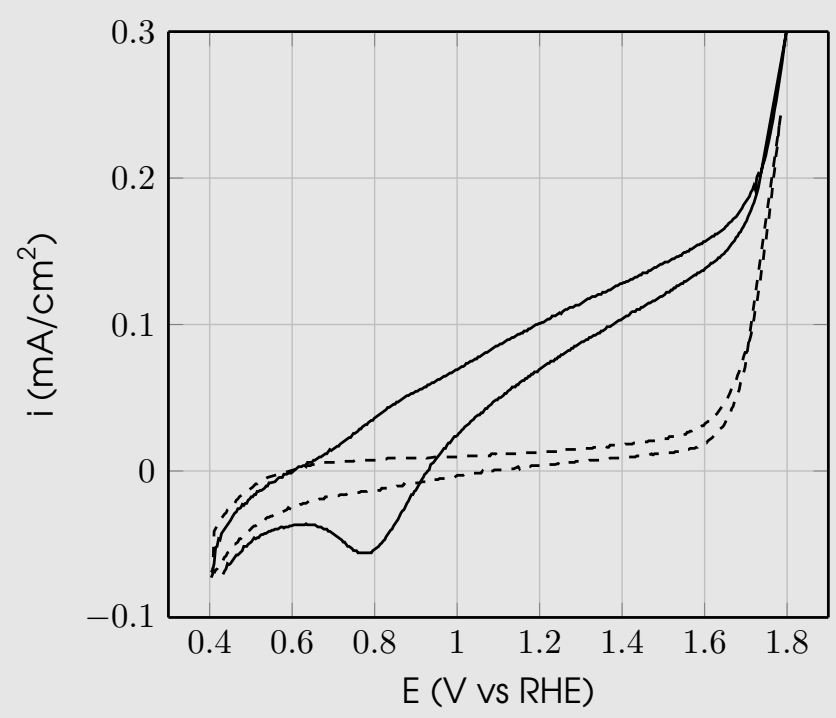

Figure 6.2: Cyclic voltammogram of a hematite layer prepared using electrophoretic deposition, in dark (dashed) and under 1 sun AM 1.5G illumination (solid).

$0.15 \mathrm{M} \mathrm{FeCl}_{3} \cdot 6 \mathrm{H}_{2} \mathrm{O}$ and $1 \mathrm{M} \mathrm{NaNO}_{3}$ in a $50 \mathrm{ml}$ stopped glass Erlenmeyer sealed with PTFE tape, with a clean $2 \cdot 2 \mathrm{~cm}^{2}$ oxidized silicon sample on which $a \sim 100 \mathrm{~nm}$ thick layer of FTO was sputtered, with the FTO side facing upwards, for $6 \mathrm{~h}$ at $100^{\circ} \mathrm{C}$. After a FeOOH layer was grown onto the FTO it was washed with deionized water and dried with $\mathrm{N}_{2}$ gas before annealing at $550^{\circ} \mathrm{C}$ for $1 \mathrm{~h}$ and at $800^{\circ} \mathrm{C}$ for $20 \mathrm{~min}$ in air. It was attempted to repeat this with well sealed $15 \mathrm{ml}$ PTFE cups with $10 \mathrm{ml}$ of the same solution but the performance was worse and the nanostructure less pronounced. The same synthesis was also performed on microstructured silicon substrates on which a FTO layer was sputtered.

The resulting layer is shown in Figure 6.3(a), clearly demonstrating the inherent nanostructure. The growth was also nicely conformal on microstructured substrates, as shown in Figure 6.3(b-d). The photocatalytic activity of the flat samples was determined in the same way as for the samples prepared by electrophoretic deposition (Section 6. 1. 1), and the result is shown in Figure 6.4. A photocurrent of almost $1 \mathrm{~mA} \mathrm{~cm}-2$ 


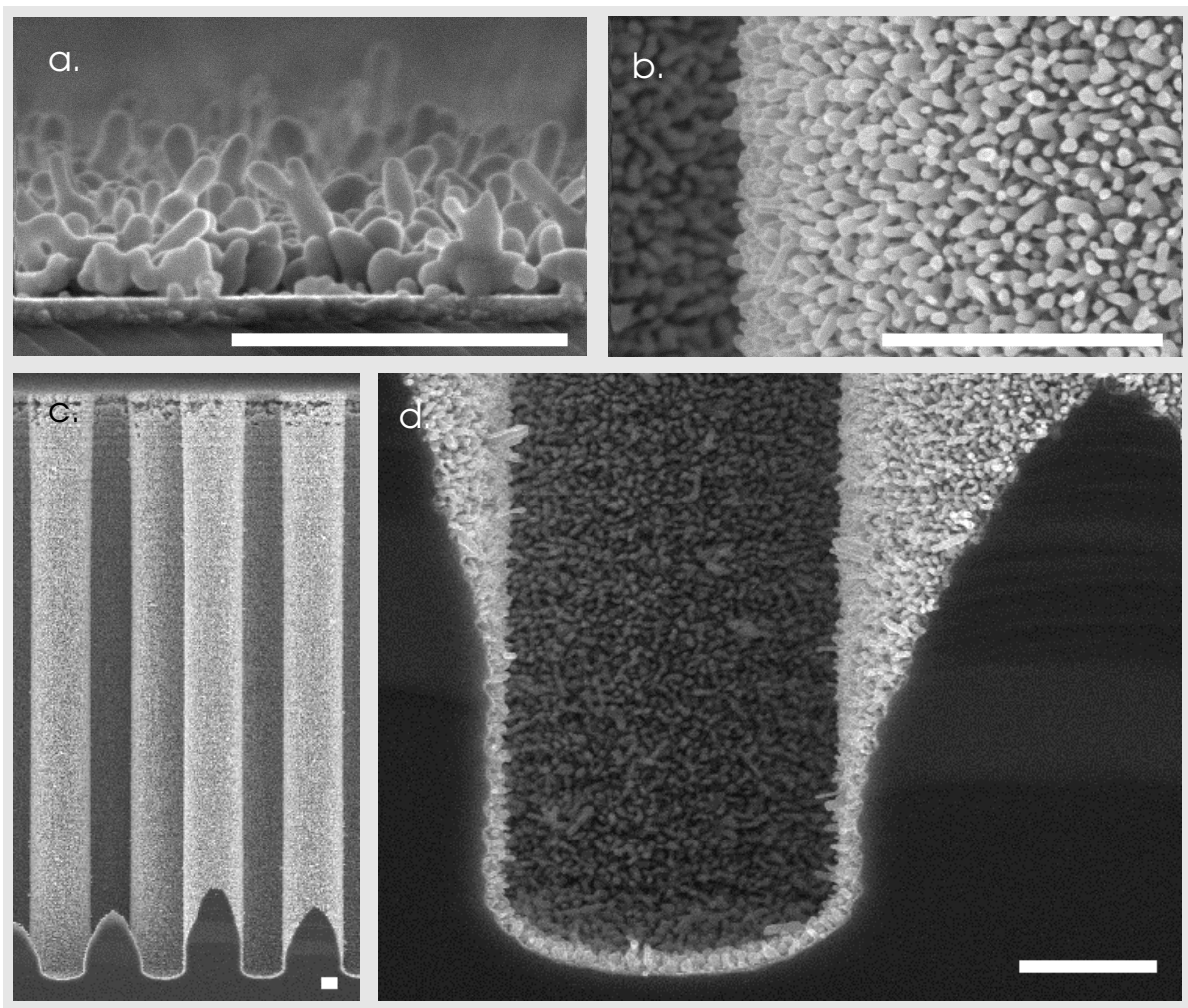

Figure 6.3: Cross-sectional SEM picture of a hematite layer prepared by hydrothermal growth in a $\mathrm{FeCl}_{3} / \mathrm{NaNO}_{3} / \mathrm{H}_{2} \mathrm{O}$ mixture at $100^{\circ} \mathrm{C}$, after annealing, (a) on a flat substrate, (b-d) on silicon micropillars. Scale bars are $1 \mu \mathrm{m}$.

at $1.23 \mathrm{~V}$ vs RHE was observed. Again the presence of a reduction peak around $0.8 \mathrm{~V}$ vs RHE in the voltammogram recorded under illumination, as well as the reasonable catalytic activity in the dark may indicate the presence of impurities.

The tested synthesis method resulted in a higher performance than the hematite layers produced by electrophoretic deposition (Section 6. 1. 1), and was found to be suitable for conformal growth on microstructured substrates. On the other hand, the potential for further performance improvement is lower: the synthesis method is based on re- 


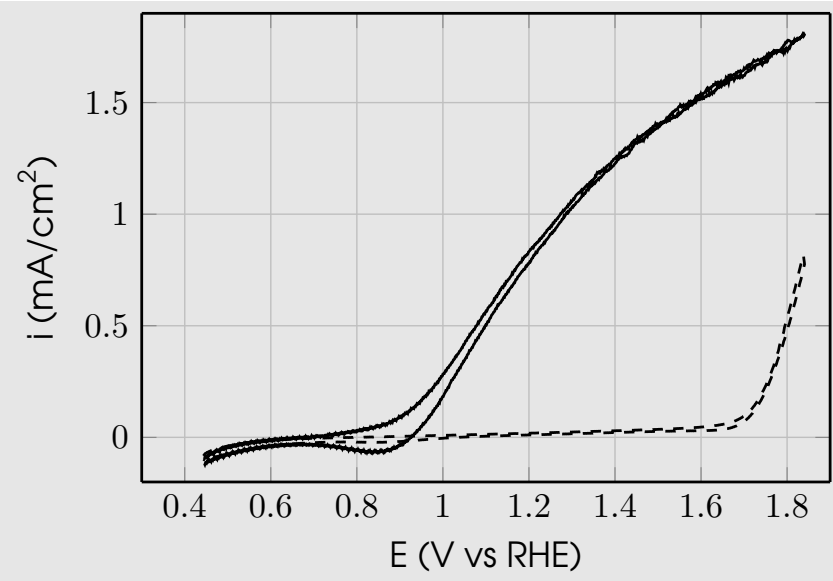

Figure 6.4: Cyclic voltammogram of a hematite layer prepared by hydrothermal growth, in dark (dashed) and under 1 sun AM 1.5G illumination (solid).

ported optimized parameters, and several improvements like catalyst deposition, doping and heterojunction formation were tried, but they did not result in improved performance.

\subsubsection{Reactive sputtering}

Hematite photoanodes have been prepared by reactive magnetron sputtering from an iron target before ${ }^{133-135}$, where Bohn et al. ${ }^{134}$ achieved a photocurrent of $0.8 \mathrm{~mA} \mathrm{~cm}^{-2}$ under 1 sun AM $1.5 \mathrm{G}$ illumination. In this method, sputtering is performed in a mixed atmosphere of argon and oxygen, at room temperature or $300^{\circ} \mathrm{C}$, and the sample is annealed afterwards at $500-800^{\circ} \mathrm{C}$ in air.

For our synthesis, highly doped p-type silicon wafers (surface concentration $\sim 1 \times 10^{21}$ boron atoms per $\mathrm{cm}^{3}$ ) were used as substrates. Native silicon oxide was removed in $1 \%$ aqueous HF solution for $1 \mathrm{~min}$, followed by rinsing with DI water and drying. Using a pure argon atmosphere, $\sim 100 \mathrm{~nm}$ of $\mathrm{Ti}_{99} \mathrm{Nb}_{1}$ was sputtered on the back side of the wafer, and $\sim 20 \mathrm{~nm}$ on the front side, each time after a base pressure of $1 \times 10^{-6} \mathrm{mbar}$ was reached. Subsequently iron oxide was sputtered 
on the front side. 23 SCCM of argon and 7 SCCM of oxygen were introduced into the chamber, and the pressure was kept at $7 \times 10^{-3} \mathrm{mbar}$. Sputtering was done from a 2 inch pure iron target with $200 \mathrm{~W}$ DC magnetron sputtering. Prior to deposition on the substrate, sputtering was performed for $1 \mathrm{~min}$ on a metal shutter plate. To achieve a titanium doping in some of the hematite layers, a $14 \mathrm{~W}$ DC plasma was used at the same time at the 4 inch $\mathrm{Ti}_{99} \mathrm{Nb}_{1}$ target. An iron oxide film thickness of $\sim 100 \mathrm{~nm}$ was achieved in $60 \mathrm{~min}$, as determined by cross-sectional SEM. After deposition, the wafer was loaded into a nitrogen atmosphere tube furnace at $400{ }^{\circ} \mathrm{C}$, and annealed at $500^{\circ} \mathrm{C}$ for $1 \mathrm{~h}$, and unloaded again at $400^{\circ} \mathrm{C}$. The wafer was broken into $2 \cdot 2 \mathrm{~cm}^{2}$ samples, and cyclic voltammetry was performed in the dark and under 1 sun AM $1.5 \mathrm{G}$ illumination in the same way as for the electrophoretically and hydrothermally grown hematite films, except that a circle of $1.6 \mathrm{~cm}$ diameter was exposed to the electrolyte and the light, and special care was taken to clean the cell with $\sim 10 \%$ aqueous nitric acid solution for half an hour and rinsing with Milli- $Q$ water before characterization, to remove metal impurities.

On some samples, an electrocatalyst was deposited by electrodeposition, to improve the water oxidation kinetics on the hematite surface. An electrolyte was prepared consisting of $6 \mathrm{mM}$ cobalt(II) nitrate hexahydrate and $6 \mathrm{mM}$ iron(III) nitrate nonahydrate in Milli- $Q$ water ${ }^{136}$. The devices were clamped to a $9 \mathrm{ml}$ cell with a circular side opening of $1.6 \mathrm{~cm}$ diameter and a platinum mesh as a counter electrode. The back side of the device was contacted by clamping a stainless steel plate to it, and a constant current of $4.93 \mathrm{~mA}\left(2.45 \mathrm{~mA} \mathrm{~cm}^{-2}\right)$ was applied with the sample as the cathode for $2 \mathrm{~min}$. During deposition the sample was illuminated by a $30 \mathrm{~W}$ white light LED placed at $\sim 3 \mathrm{~cm}$ from the sample, to improve the conductivity of the hematite. The cell was emptied and the device removed, and rinsed thoroughly with Milli- $Q$ water. Cyclic voltammetry in light and dark was performed with only a circle of $1.2 \mathrm{~cm}$ diameter exposed to the electrolyte and the light.

Results for the fifth cycle of the voltammetry of several samples are shown in Figure 6.5. As can be seen the titanium doping and catalyst addition clearly improve the device performance. Unfortunately in later fabrication runs photoactive samples could no longer be produced. The performance of an example device, fabricated in the same way (without doping or catalyst), is also shown in Figure 6.5.

Photoactive and inactive undoped samples without a catalyst were 


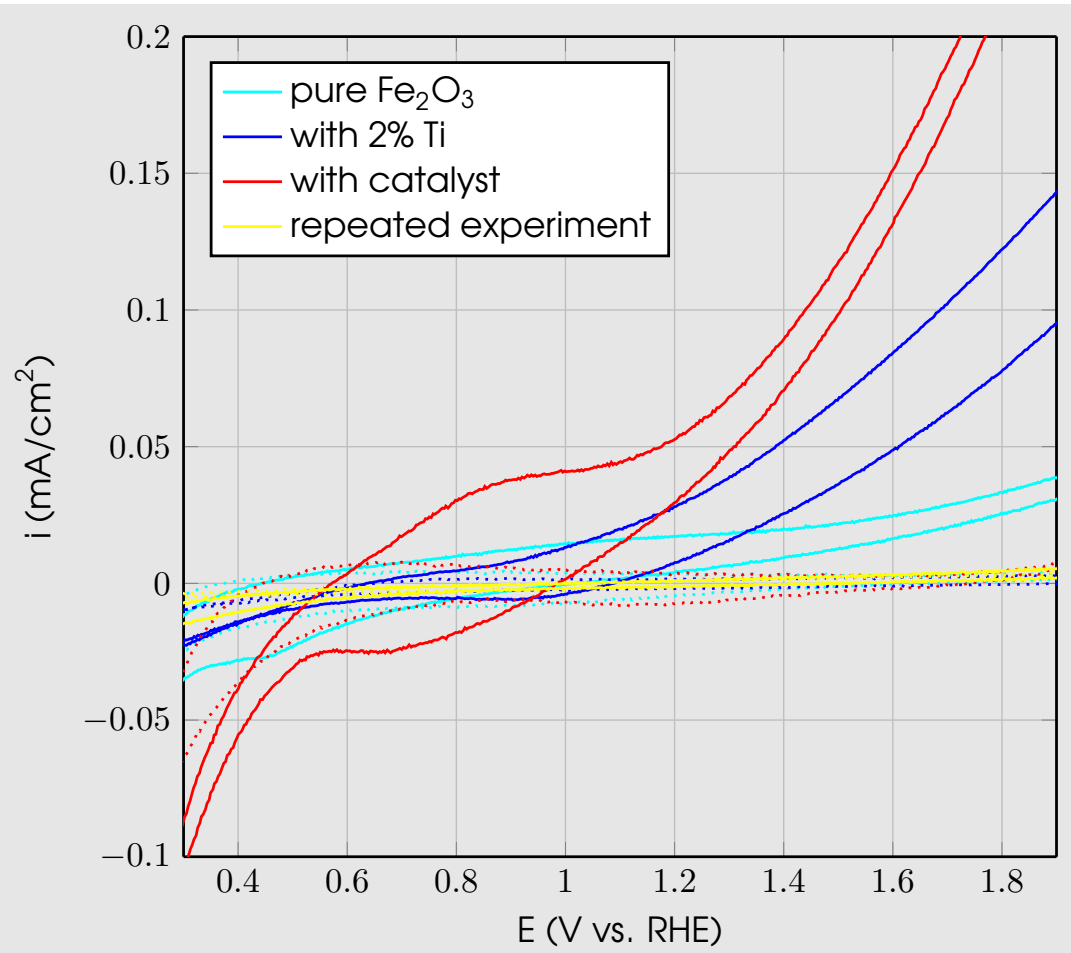

Figure 6.5: Cyclic voltammograms of hematite layers prepared by sputtering, in dark (dotted) and under 1 sun AM 1.5G illumination (solid).

analyzed by XRD and XPS. XRD was also performed on a sample that was treated in the same way (silicon doping, $\mathrm{Ti}_{99} \mathrm{Nb}_{1}$ deposition, annealing) except that no iron oxide was deposited. XRD results are shown in Figure 6.6. The peaks at $33,39,54,56$ and $61^{\circ}$ are attributed to the substrate. The photoactive sample XRD spectrum is practically the same as the substrate spectrum, with no signs of crystalline iron oxide, except for a small peak around $36^{\circ}$, which could be the (110)-peak of hematite. The inactive sample on the other hand has a clear hematite footprint with the (012) and (024) peaks at 24 and $49^{\circ}$. The large peak at $39^{\circ}$ is smaller for this sample, and because of the XPS measurements presented below this peak is attributed to $\mathrm{TiO}_{2}$.

Survey XPS spectra were taken of the surface of both photoactive 


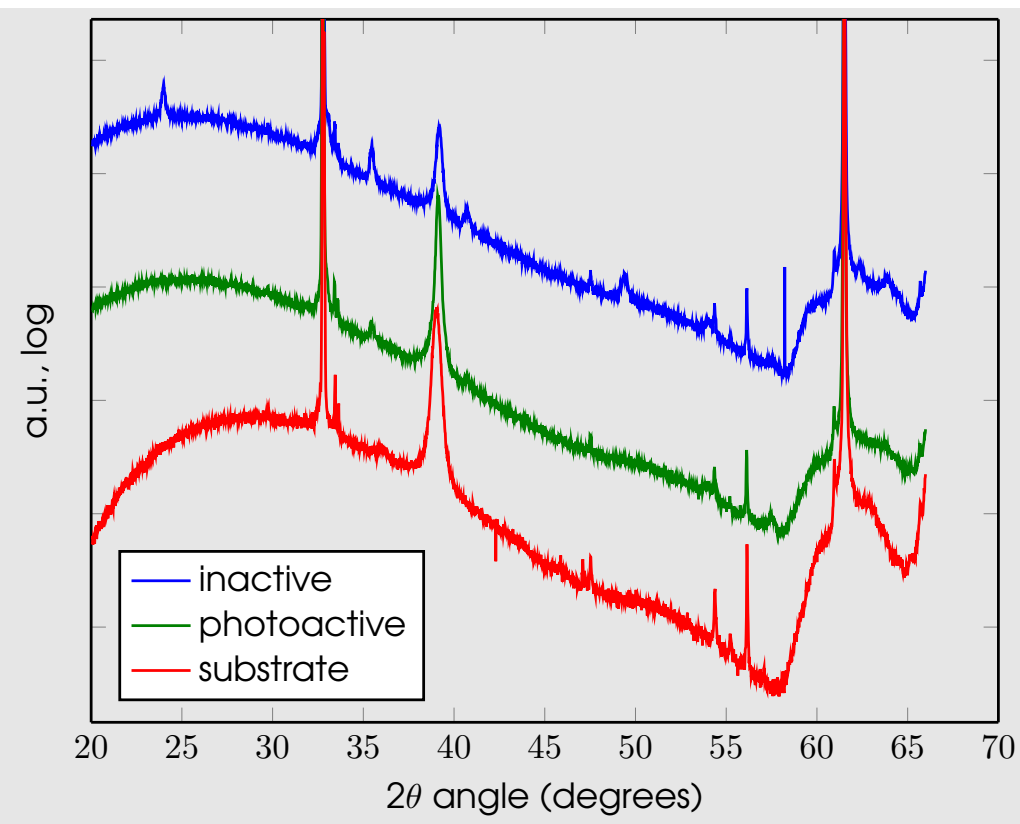

Figure 6.6: XRD spectra for several sputtered and annealed silicon$\mathrm{Ti}_{99} \mathrm{Nb}_{1}$-hematite samples.

and inactive samples, as shown in Figure 6.7. The photoactive sample seems to have a bit more carbon contamination at the surface, but no other impurity differences were observed. The almost identical Fe2p region for both samples (see Figure 6.8) indicates the identical oxidation state of the iron. The $\mathrm{Fe} 2 \mathrm{p}_{3 / 2}$ satellite peak seems to be located around $719 \mathrm{eV}$ rather than at $715 \mathrm{eV}$, suggesting an oxidation state of 3+ rather than $2+{ }^{137}$. A depth profile was then made by alternately ion milling and scanning at the relevant energies for iron, oxygen, silicon, titanium and niobium, so that a depth profile of atomic concentrations could be made. These profiles are shown in Figure 6.9. The most obvious difference is the more gradual transition from iron oxide to titanium oxide in the photoactive sample, but this could be due to a greater spread in layer thickness in the photoactive sample. Strikingly, the iron:oxygen ratio in the bulk iron oxide, 42:55, was found to be closer to $\mathrm{Fe}_{3} \mathrm{O}_{4}$ than to the $\mathrm{Fe}_{2} \mathrm{O}_{3}$ expected for hematite. Furthermore the titanium:oxygen 


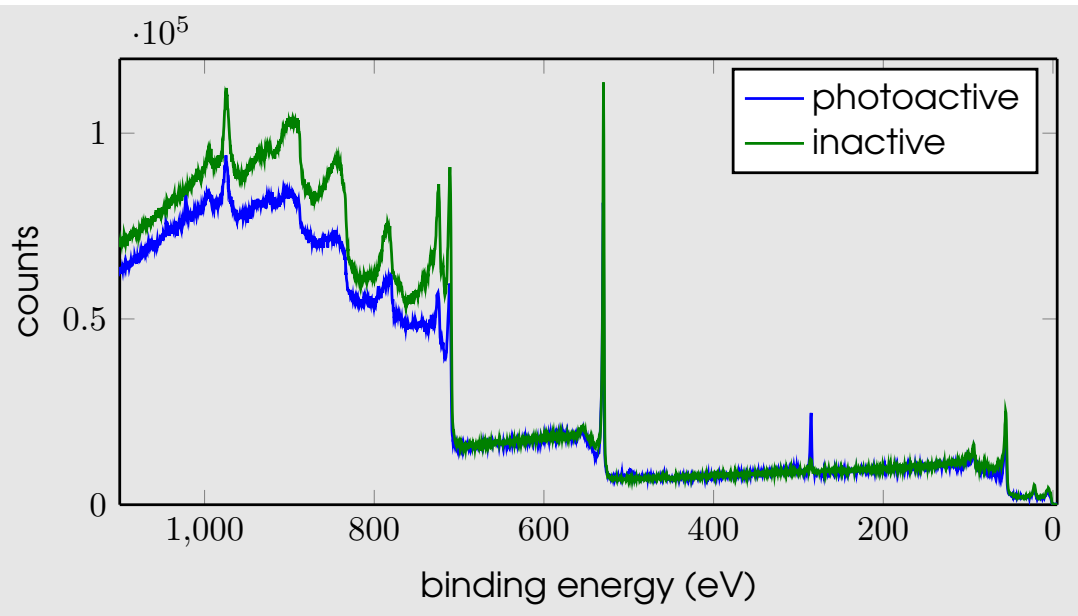

Figure 6.7: XPS survey spectra for two sputtered and annealed silicon$\mathrm{Ti}_{99} \mathrm{Nb}_{1}$-hematite samples.

ratio indicates fully oxidized titanium $\left(\mathrm{TiO}_{2}\right)$. This suggests that some oxygen was transferred from the iron to the titanium during annealing. Furthermore, the niobium doping in the titanium seems to have diffused to the interface with the silicon.

Overall, the clearest difference between the photoactive and inactive sample seemed to be the degree of crystallinity of the iron oxide, and therefore it was tried to decrease the crystallinity by decreasing the annealing temperature to $450^{\circ} \mathrm{C}$. Figure 6.10 compares the cyclic voltammetry results of such a device with the previously fabricated photoactive and inactive devices. Some waves are visible for the device annealed at a lower temperature, indicating the presence of impurities in the electrolyte. However the sample was also clearly photoactive as the currents were higher than for the measurements in the dark, confirming the necessity of limited crystallinity for these photoanodes. 


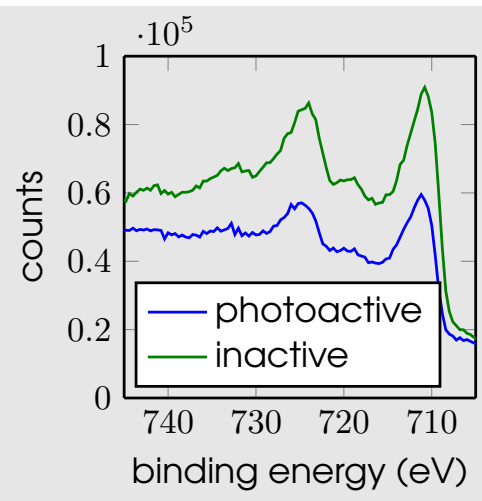

Figure 6.8: XPS spectra in Fe2p region for two sputtered and annealed silicon- $\mathrm{Ti}_{99} \mathrm{Nb}_{1}$-hematite samples.

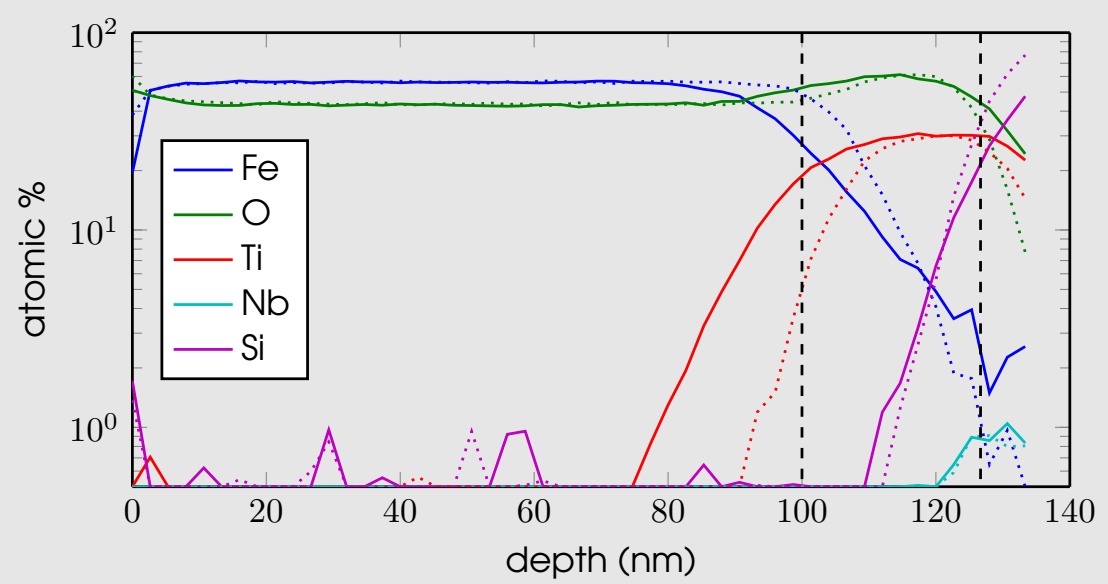

Figure 6.9: Depth profiles of XPS-derived atomic concentrations for the photoactive (solid lines) and inactive (dotted lines) silicon- $\mathrm{Ti}_{99} \mathrm{Nb}_{1}$ hematite sample. The vertical dashed lines indicate the estimated locations of the interfaces between iron oxide, titanium oxide, and silicon. 


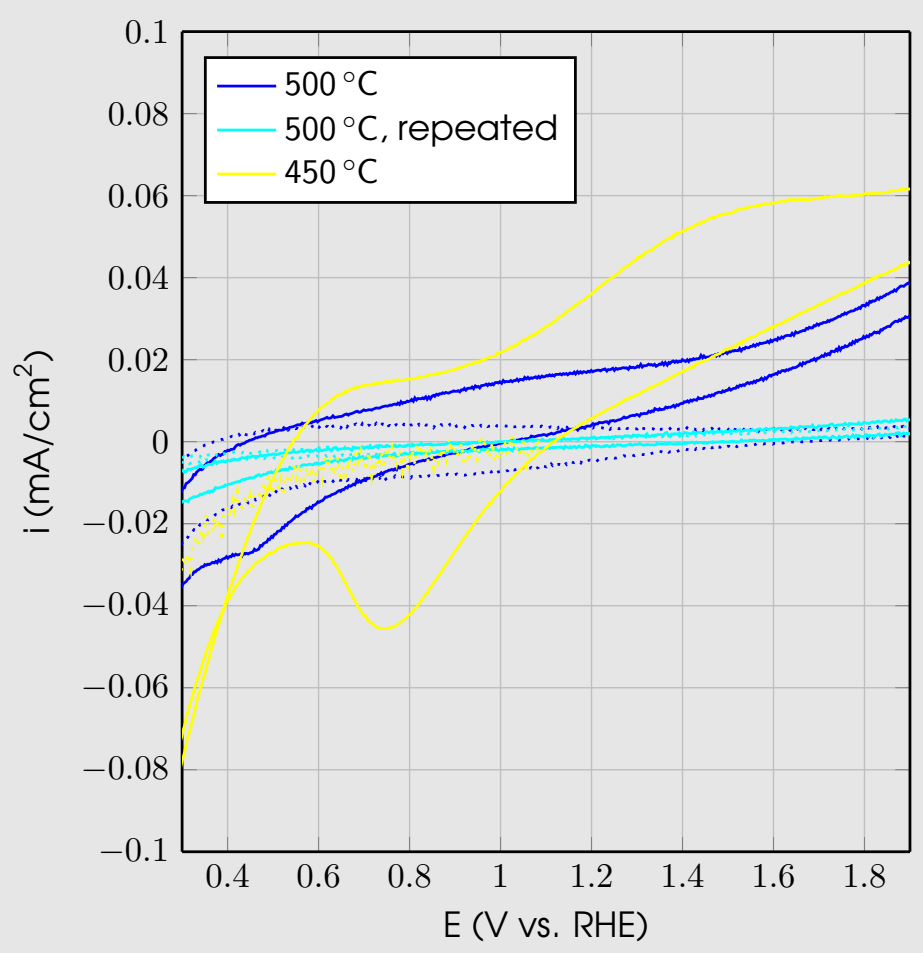

Figure 6.10: Cyclic voltammograms of hematite layers prepared by sputtering, in dark (dotted) and under 1 sun AM 1.5G illumination (solid).

\subsection{Tandem with silicon}

In a tandem cell, the total voltage is generated by the absorption of two photons instead of one. Each of these photons creates an electron-hole pair in a separate 'subcell' of the total semiconductor stack. To be able to add the voltages of both subcells together, the holes from one subcell have to recombine with the electrons from the other subcell. If the band edge energy levels and Fermi levels of the two semiconductors at this interface are aligned properly, a tunnel junction is formed where this recombination can happen, and the relative position of the band edges and the Fermi level can be influenced 
by doping the semiconductors ${ }^{138}$. It has been shown that hematite deposited directly on n-Si can form a tandem device ${ }^{139-142}$, where the silicon contributes $\sim 0.3 \mathrm{~V}$ to the photovoltage. Thin layers of hematite have also been used directly on silicon to function purely as an electrocatalyst or protection layer ${ }^{143,144}$. Although photovoltages over $0.7 \mathrm{~V}$ can be achieved with silicon p-n junctions, no attempts were found to fabricate a hematite photoanode directly on a silicon p-n junction cell. This would be more complicated since the p-type silicon would have to be at the hematite side of the cell, and the band alignment p-type silicon and hematite is such that they would not automatically form a tunnel junction.

Since the doping in the hematite could not easily be changed, it was here investigated whether the ohmic contact between the p-type silicon and the hematite could be achieved with a metallic conductor ${ }^{145}$. Illumination is envisioned to pass first through the hematite since it has a higher band gap and is transparent to some wavelengths that can still be absorbed by the silicon. However in that case the metallic conductor should not prevent the light from reaching the silicon, and therefore a transparent conductive oxide (TCO) would probably work best. Fluorine-doped tin oxide (FTO) is the most common TCO that is both made out of earth-abundant elements and stable in alkaline electrolyte. It is also the conductive substrate of choice for most studies on hematite as a photoanode. However prohibitively poor conductance was found between a sputtered layer of FTO and highly doped p-type silicon after annealing at $700^{\circ} \mathrm{C}$ and higher, which was needed for making the FTO conductive (electrical activation of the fluorine dopant) and for many hematite synthesis routes. This is probably due to the lower formation enthalpy of $\mathrm{SiO}_{2}$ compared to $\mathrm{SnO}_{2}$, so that during annealing the tin oxide is reduced while the silicon underneath it is oxidized, forming an insulating layer. Kurtz and Gordon ${ }^{146}$, who have analyzed this problem, suggest the use of (doped) $\mathrm{TiO}_{2}$ as an interlayer between silicon and $\mathrm{FTO}$. $\mathrm{TiO}_{2}$ has a lower enthalpy of formation than $\mathrm{SiO}_{2}$ and therefore oxygen is not expected to move from the $\mathrm{TiO}_{2}$ to the silicon, although oxygen from the annealing atmosphere might still diffuse to the silicon and oxidize it.

Niobium-doped $\mathrm{TiO}_{2}$ (NTO) is a TCO itself ${ }^{147}$ and it was therefore investigated as a replacement for FTO for this application. It was reactively sputtered from a $\mathrm{Ti}_{99} \mathrm{Nb}_{1}$ target ${ }^{148}$ in the same way as $\mathrm{TiO}_{2}$ films were prepared before on the sputtering machine that was used ${ }^{149,150}$. 


\subsubsection{Electrical contact and transparency evaluation}

NTO was tested as a TCO layer on top of a flat solar cell. To make the solar cell, a silicon wafer (n-type, $1-10 \Omega \mathrm{cm}, 10 \mathrm{~cm}$ diameter, singleside polished, OKMETIC), was cleaned in an ozone/steam atmosphere, and subsequently in $1 \%$ aqueous $\mathrm{HF}$ solution, rinsed thoroughly in $\mathrm{DI}$ water, and finally dried. A phosphorus dopant source layer was deposited by LPCVD (300 nm thick phosphosilicate glass, $350 \mathrm{mTorr}, 675^{\circ} \mathrm{C}$, $30 \mathrm{SCCM} \mathrm{N} \mathrm{N}_{2}$ through the tetraethyl orthosilicate (TEOS) evaporator to form a $50 \mathrm{SCCM}$ TEOS/ $\mathrm{N}_{2}$ mixture, $150 \mathrm{SCCM} \mathrm{O}_{2}, 16.5 \mathrm{SCCM} \mathrm{PH} \mathrm{S}_{3}$ and 313.5 SCCM argon, $30 \mathrm{~min})$, followed by a silicon oxide capping layer $(100 \mathrm{~nm}$ thick, $350 \mathrm{mTorr}, 675^{\circ} \mathrm{C}, 30 \mathrm{SCCM} \mathrm{N}$ through the TEOS evaporator to form a $50 \mathrm{SCCM}$ TEOS $/ \mathrm{N}_{2}$ mixture, $10 \mathrm{~min}$ ). The unpolished back side of the wafer was covered with photoresist (Olin OIR 907-17) using spin coating and baked at a hotplate at $120^{\circ} \mathrm{C}$ for $5 \mathrm{~min}$. The wafer was then immersed for $2 \mathrm{~min}$ in $12.5 \% \mathrm{HF}$ in $\mathrm{NH}_{4} \mathrm{~F}$ ( $\left.1: 7 \mathrm{BHF}\right)$ to remove the dopant layer from the front side, followed by rinsing, and then the photoresist layer was stripped in room temperature $99 \%$ nitric acid for $10 \mathrm{~min}$, rinsing and drying.

Subsequently, solid source doping was performed. The wafers were cleaned in room temperature $99 \%$ nitric acid, $95^{\circ} \mathrm{C} 69 \%$ nitric acid and $1 \%$ aqueous HF solution subsequently, rinsed thoroughly in DI water between each step, and finally dried. They were then placed in a furnace with the top side of the wafer facing (but not touching) a boron nitride wafer which served as a source of boron. A layer of boron oxide was grown on the wafers at $900^{\circ} \mathrm{C}$ in an atmosphere of 2 standard liters per minute (SLM) $\mathrm{N}_{2}$ and $2 \mathrm{SLM} \mathrm{O}_{2}$ for $15 \mathrm{~min}$, and the atmosphere was changed to only $\mathrm{N}_{2}$ (4SLM) and the temperature of the furnace was further increased to $1050^{\circ} \mathrm{C}$. It remained in that condition for $15 \mathrm{~min}$ to diffuse the boron from the boron oxide and the phosphorus from the phosphosilicate glass into the silicon, and then it was cooled down again and removed from the furnace. The boron oxide, silicon oxide and phosphosilicate glass were removed by etching for $10 \mathrm{~min}$ in buffered HF solution, followed by thorough rinsing and drying. Any boron silicide that might have formed was oxidized for $15 \mathrm{~min}$ at $800^{\circ} \mathrm{C}$ in oxygen, and the resulting oxide was removed by etching another $10 \mathrm{~min}$ in buffered HF solution, followed by rinsing and drying. In this way a highly doped n-type layer was formed on the back side to improve the electrical contact with the back side contact, and a highly doped 
p-type region was made on the front side to form a p-n junction with the bulk of the wafer.

Any native oxide on the silicon was removed in $1 \%$ aqueous HF solution, followed by rinsing and drying, and immediately after that a back side contact of $\sim 100 \mathrm{~nm}$ of $\mathrm{Ti}_{99} \mathrm{Nb}_{1}$ was sputtered from the same target in a pure argon atmosphere, followed by reactively sputtering $\sim 65 \mathrm{~nm}$ of NTO in $40 \mathrm{~min}$ on the front side. A $500 \mathrm{~W}$ DC magnetron plasma was used with gas flows of 30 SCCM argon and 6 SCCM oxygen at a pressure of $7 \times 10^{-3}$ mbar. Aluminum contacts were sputtered on the front side, and patterned using lithography and wet etching, followed by photoresist removal in $99 \%$ nitric acid. These solar cells were characterized under AM 1.5G simulated solar illumination, and the results are shown in Figure 6.11 (blue line, 'no Ti').

The low slope close to open circuit conditions indicates that the device behavior is dominated by a series resistance, which is presumably a resistance between the NTO and the silicon. Therefore, inspired by the work of Kast et al. ${ }^{151}$, similar devices were made again, but with a metallic layer, sputtered in pure argon, added between the silicon and the NTO, with several thicknesses. They were measured in the same way, and the $i$ - $V$ curves for two $\mathrm{Ti}_{99} \mathrm{Nb}_{1}$ layer thicknesses shown in Figure 6.11 (green line, ' $3 \mathrm{~nm} \mathrm{Ti',} \mathrm{and} \mathrm{red} \mathrm{line,} \mathrm{'} 10 \mathrm{~nm} \mathrm{Ti}$ '). Clearly a few nanometers of $\mathrm{Ti}_{99} \mathrm{Nb}_{1}$ already gave a tremendous increase in performance of the solar cell. Further increasing the $\mathrm{Ti}_{99} \mathrm{Nb}_{1}$ layer thickness did not lead to any relevant improvement in contact resistance, but instead lowered the photocurrent, presumably because of parasitic light absorption/reflection.

The most severe annealing to which hematite is subjected in all the synthesis methods that were tried was annealing for $1 \mathrm{~h}$ at $800^{\circ} \mathrm{C}$ in oxygen or air atmosphere. However, even $a \sim 1 \mu \mathrm{m}$ thick layer of $\mathrm{Ti}_{99} \mathrm{Nb}_{1}$ was found to oxidize completely under these circumstances, and therefore oxidation of the silicon underneath is also expected. Therefore the solar cells as described above were subjected to the same annealing (directly after the reactive sputtering of NTO, so before the aluminum front side contact formation), with the exception that a nitrogen atmosphere was used. Wafers were loaded and unloaded while the tube furnace was at $400^{\circ} \mathrm{C}$. These devices were also measured as solar cells as above, and the results for two $\mathrm{Ti}_{99} \mathrm{Nb}_{1}$ thicknesses are also shown in Figure 6.11 (cyan line, ' $10 \mathrm{~nm} \mathrm{Ti}$, annealed', and purple line, ' $30 \mathrm{~nm} \mathrm{Ti}$, annealed'). Clearly, even for a $10 \mathrm{~nm}$ thick 


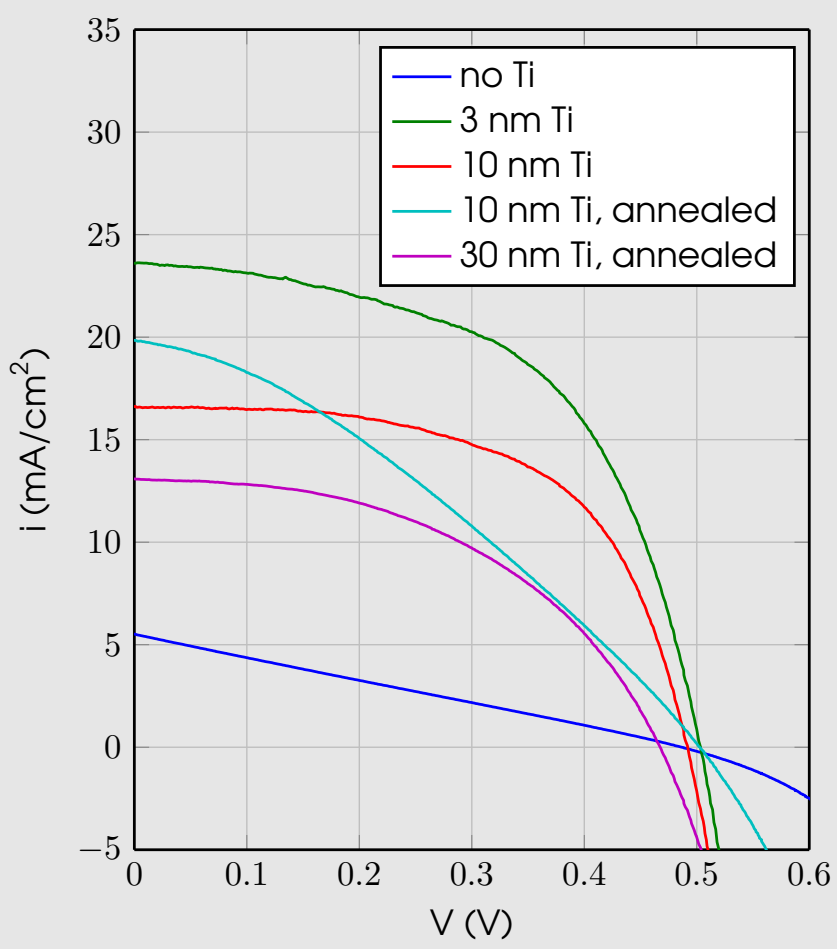

Figure 6.11: Current-voltage relations for solar cells with various conductive overlayers, average currents from measurements on four devices per type.

$\mathrm{Ti}_{99} \mathrm{Nb}_{1}$ layer, the contact resistance and photocurrent had increased with respect to the unannealed devices, suggesting that the $\mathrm{Ti}_{99} \mathrm{Nb}_{1}$ interlayer was oxidized at least to some extent, despite the nitrogen atmosphere. This is in good agreement with the XPS depth profiles for sputtered hematite samples annealed in nitrogen (Figure 6.9), which showed a Ti:O ratio of $1: 2$. This oxygen may have originated from the oxide layers on top, from oxygen impurities in the nitrogen flow, or have been introduced into the furnace during loading (meaning that the oxidation would already occur at $400^{\circ} \mathrm{C}$ because later in the annealing process this would have been flushed out by the constant nitrogen flow), or the layer may have oxidized in the air upon unloading. 
For a thicker $(30 \mathrm{~nm}) \mathrm{Ti}_{99} \mathrm{Nb}_{1}$ layer the contact resistance did not seem to increase as much, but the device was now more limited by the decreased photocurrent, and therefore the photovoltage at 10 $\mathrm{mA} \mathrm{cm}^{-2}(290 \mathrm{mV})$ was actually lower than for the $10 \mathrm{~nm} \mathrm{Ti}{ }_{99} \mathrm{Nb}_{1}$ layer $(316 \mathrm{mV})$.

Overall, an interlayer was developed between silicon and an oxide layer integrated on top of it, which can provide a low ohmic contact between the layers. It consists only of earth-abundant elements, and a layer thickness of at least $10 \mathrm{~nm}$ was found to be necessary if the device is not to be limited by the contact resistance when annealing at $800^{\circ} \mathrm{C}$ in nitrogen. At this temperature, an oxygen atmosphere led to too much oxidation. For lower temperatures the inert atmosphere may not be necessary, or perhaps an even thinner $\mathrm{Ti}_{99} \mathrm{Nb}_{1}$ layer can be used, but this was not investigated since with the state-of-the-art photoanode made of earth-abundant elements the device photocurrent would anyway be limited by the photoanode and not by the silicon.

\subsubsection{Tandem devices}

The synthesis of hematite was tried on highly conductive silicon substrates with the $\mathrm{Ti}_{99} \mathrm{Nb}_{1}$ interlayer using electrophoretic deposition as in Section 6.1.1 and hydrothermal growth as in Section 6.1.2, with the exception that the annealing step was performed in nitrogen atmosphere. However, no photoactivity was found for these devices. Since both synthesis methods depend on a FeOOH precursor, it was hypothesized that this is not properly converted to hematite in a nitrogen atmosphere. This was confirmed by repeating the experiments on FTO samples, which led to photoactive layers before, but again with the exception that annealing was performed in nitrogen atmosphere, and again no photoactivity was found.

Therefore the hematite was deposited directly on the $\mathrm{Ti}_{99} \mathrm{Nb}_{1}$ layer using reactive sputtering as described in Section 6. 1.3, since this method already introduces sufficient oxygen into the layer before annealing. This was done when annealing at $500^{\circ} \mathrm{C}$ still led to photoactive hematite layers, and no dopant or catalyst was used. Figure 6.12 shows the results of the photoelectrochemical characterization of samples that were prepared on silicon substrates with and without a p-n junction, 


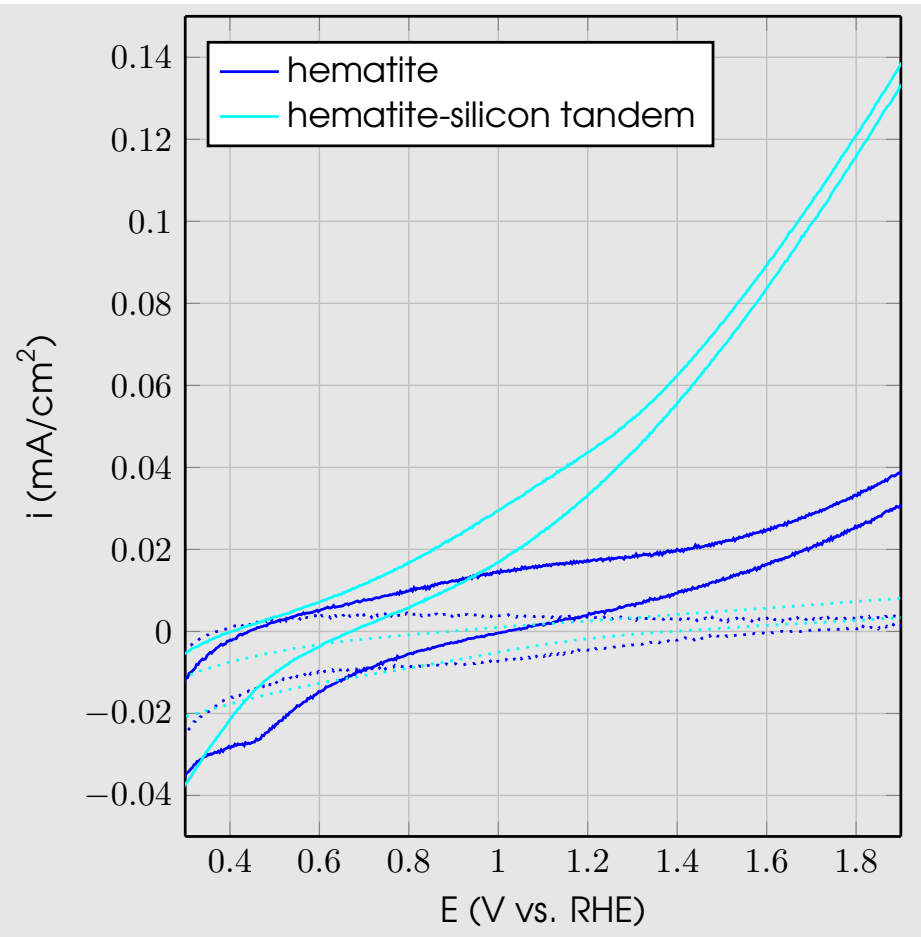

Figure 6.12: Cyclic voltammograms of hematite layers prepared by sputtering when a substrate with and without a p-n junction was used, in dark (dotted) and under 1 sun AM 1.5G illumination (solid).

showing the obvious improvement achieved by adding a solar cell underneath the hematite photoanode. However the characteristic of the tandem device is not a simple cathodic shift of the hematite-only characteristic, showing that the combined behavior cannot be explained by only describing the solar cell as a constant voltage source. There seems to be a cathodic shift of the onset voltage, but because of the difference between the forward and backward CV scan, which is also different for both types of devices, this is hard to quantify. 


\subsection{Conclusion}

Three synthesis principles for hematite were investigated in this Chapter: electrophoretic deposition, hydrothermal growth and reactive sputtering. Of these methods, hematite photoanodes synthesized using hydrothermal growth have so far shown the highest photocurrents. However it has to be mentioned that for none of the synthesis methods all relevant processing parameters were optimized, and none of them has come close to the record performance reported for these methods in the literature. Therefore further optimization may shift this conclusion. Also numerous possible improvements for hematite have been suggested in literature, like (gradient/homogeneous) doping, addition of an electrocatalyst/passivation layer or plasmonic particles/layers, heterojunction formation and structuring ${ }^{125}$. The final optimized performance of hematite photoanodes formed by each of the tried synthesis methods may depend critically on the extent to which these possible improvements are compatible with that particular synthesis method, and this may again lead to a different conclusion about which synthesis method is most suitable.

An integrated silicon-hematite photoanode was successfully fabricated with sputtered hematite, and showed improved performance over the hematite-only photoanode. However it did not produce enough photovoltage to independently split water. The performance of the hematite photoanode used was poor compared to record devices reported in literature, and this is limiting the device behavior. Therefore improvements of the hematite performance are expected to directly lead to improvements in the tandem device performance. Also the voltage added by the silicon $(300-400 \mathrm{mV}$ ) is only about half of the $750 \mathrm{mV}$ that has already been shown ${ }^{152}$ for silicon cells. Partially this is due to the relatively simple silicon cell that was used, but also to the $\mathrm{Ti}_{9 g} \mathrm{Nb}_{1}$ used as an interlayer to make electrical contact between the silicon and the hematite, for which there was a trade-off in the layer thickness between contact resistance and light reflection. The thickness and niobium content of this layer was not optimized for the specific annealing conditions used for the synthesis of the hematite layer. Better tuned annealing schemes (time, temperature, oxygen content, multiple steps, perhaps rapid thermal annealing) may help to improve the performance of the interlayer or make it compatible with other hematite synthesis methods. 
Since no hematite photoanodes were found in literature with large photocurrents below the potential of $750 \mathrm{mV}$ provided by the best silicon cell, it remains doubtful if a tandem formed by these materials will ever be able to split water at significant rates without an external bias. To achieve this probably a triple junction device or a higher band gap photoanode material will have to be used, and both would lower the theoretical maximum efficiency of the device ${ }^{7}$. 


\section{Chapter 7}

\section{Solar-to-fuel device concept: a thin perforated silicon membrane}

In Chapter 2, and especially Section 2.4, a device concept was discussed where a 'wireless' solar water splitting device was perforated with numerous small holes to improve ion transport without compromising the light absorption and gas separation too much. In this chapter the fabrication and testing of such devices is described in detail.

\subsection{The device concept}

The envisioned standalone solar water splitting membrane is presented in Figure 7.1. The structure and structural strength are mainly provided by a crystalline silicon membrane, perforated with a regular array of pores. For the devices that were made the thickness of the membrane was around $40 \mu \mathrm{m}$ and the diameter of the holes was around $3 \mu \mathrm{m}$, but these dimensions are not crucial for this concept. The silicon also acts as a light absorber and contains a $\mathrm{p} / \mathrm{n}$-junction ( $\mathrm{p}$-type at the top and n-type at the bottom) to separate the generated charges. 


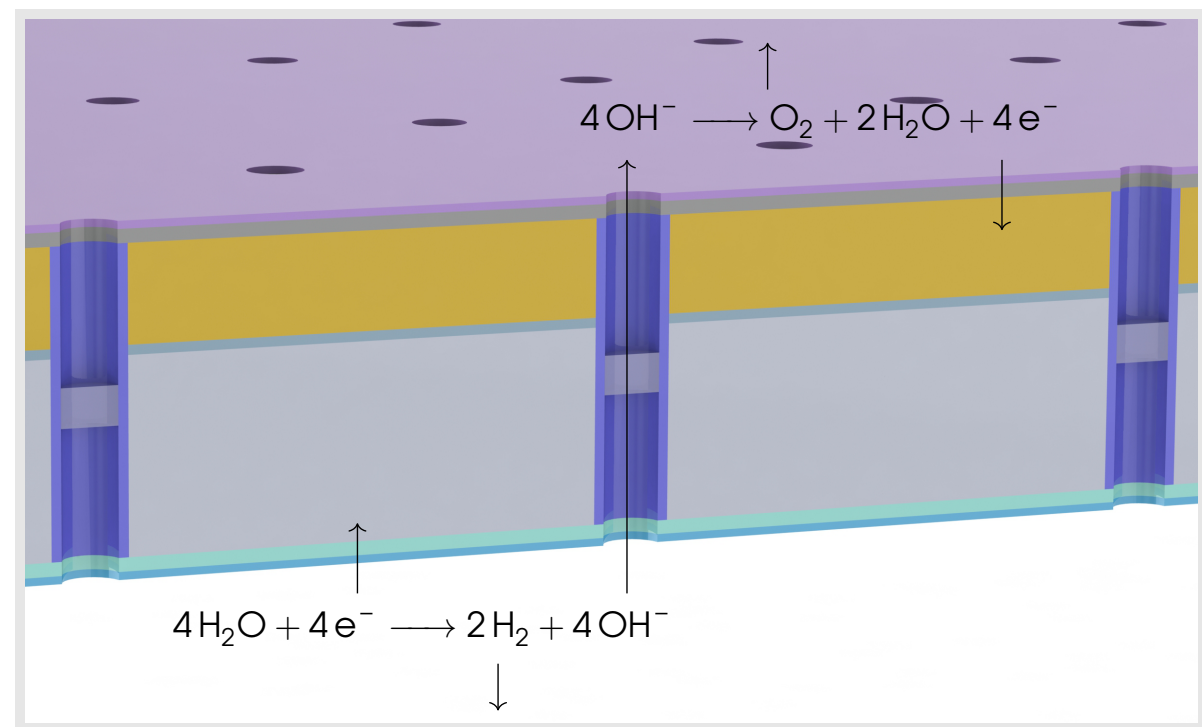

Figure 7.1: Cross-sectional view of the envisioned device structure presented in this chapter. The basis is a silicon membrane (light gray) with pores. On the bottom, cathode side it could have protection and catalyst layers. On the top, anode side it can have layers of light absorbers (orange), maybe with a layer for ohmic contact with the silicon, and with protection and or catalyst layers on top. The inside of the pores can be protected and/or passivated with another layer (blue), and the pores can be partially filled with an ion selective membrane material (gray, transparent).

On the bottom, cathode side the device could have (conductive) protection and catalyst layers, which could be opaque since the light is entering from the top, anode side.

On the top side the device can have layers of light absorbers to provide the additional voltage needed for water splitting. The topmost absorber layer can have a semiconductor-electrolyte junction for the charge separation. At the interface between absorber layers an ohmic contact has to be created, by creating high doping concentrations and perhaps a metallic conductor interlayer. On top of the topmost semiconductor there has to be a transparent or patterned catalyst, and if this semiconductor is not stable in the electrolyte used, 
a protection layer may be necessary.

The inside of the pores may also have to be coated with a protection layer if one (or more) of the semiconductors or ohmic contact layers is not stable in the electrolyte used. The pores can also be filled (partially) with a membrane material that helps to separate the product gases.

Perhaps not all of these elements are necessary for the final combination of materials and dimensions. Indeed it is shown in this Chapter that the gas separation is sufficient without the membrane material for the dimensions and current densities chosen.

This device concept was inspired by the work of Bosserez et al. ${ }^{17}$, Hankin et al. ${ }^{19}$ and Trompoukis et al. ${ }^{18}$, which presented some notable improvements in the trade-off between ion transport and light absorption, as discussed in Chapter 2. However some important features and improvements are added or combined in the device presented here:

- Light absorption by the silicon and additional semiconductor layers is included, similar to the work by Hankin et al. ${ }^{19}$ and Trompoukis et al. ${ }^{18}$, so that the device can generate the current necessary for water splitting without an externally applied voltage.

- The membrane is made thinner, so that the ion transport distance is further reduced.

- The trend of going from a few big holes to a lot of small holes in the work of Bosserez et al. ${ }^{17}$ is continued further, to further reduce the ion transport distances. Trompoukis et al. ${ }^{18}$ investigated this by using simulations, here it is investigated experimentally.

- By making the holes smaller, gas separation is improved.

- Protection layers are added inside the pores, to improve device stability.

- These protection layers also passivate the defective silicon surface inside the holes, to improve electrical performance.

- Only earth-abundant materials are used.

Most of these improvements were realized in actual devices. The methods and results are described in this chapter. Only the standalone 
water splitting upon solar illumination was not achieved. The addition of an extra absorber layer to the silicon is studied to some extent in Chapter 6, but this was not yet tested in the membrane configuration presented in the present chapter. Therefore an external bias was always necessary, and most of the experiments described in this chapter were performed without solar illumination.

\subsection{Materials and methods}

\subsubsection{Electrolyzer membrane fabrication}

Perforated silicon membranes for use as electrolyzers (so without photovoltaic capabilities) were made as summarized in Figure 7.2. The process is described here in detail, with the lowercase letters in round brackets referring to the respective step in the figure. Except for the breakout of the devices, electrodeposition of the catalysts, and removal of the photoresist and metal from the pores in steps ( $k)$ and (I), all steps were performed inside a cleanroom environment.

(a) The starting point was a silicon wafer (n-type, $1-10 \Omega \mathrm{cm}, 10 \mathrm{~cm}$ diameter, single-side polished, OKMETIC), which was cleaned in room temperature $99 \%$ nitric acid and $95{ }^{\circ} \mathrm{C} 69 \%$ nitric acid subsequently, and thoroughly rinsed in DI water. Finally the silicon oxide formed in these steps was stripped by etching for $1 \mathrm{~min}$ in $1 \%$ aqueous HF solution, followed by rinsing and drying. (b) A $1 \mu \mathrm{m}$ silicon oxide hard mask was formed by wet thermal oxidation at $1150^{\circ} \mathrm{C}$ for $137 \mathrm{~min}$. A hexagonally packed pattern of $3 \mu \mathrm{m}$ diameter holes was applied on the polished side using Olin OIR 907-17 positive resist and optical lithography, to define nine hole fields of 2.2 by $2.2 \mathrm{~cm}$. The hole center-to-center distance was varied from 14 to $229 \mu \mathrm{m}$ to achieve porosities ranging from $4 \%$ down to $0.016 \%$. This pattern was transferred to the silicon oxide by RIE (Oxford Plasmatherm 790 parallel plate etcher, with graphite electrode, $27 \mathrm{SCCM} \mathrm{CHF}_{3}, 3 \mathrm{SCCM} \mathrm{O}_{2}$, pressure $20 \mathrm{mTorr}$ and power $350 \mathrm{~W}$ for $50 \mathrm{~min}$ ). This hard mask was used to etch approximately $40 \mu \mathrm{m}$ straight down in the silicon (SPTS Pegasus, Bosch recipe with 267 cycles, deposition: $0.6 \mathrm{~s}$ with $150 \mathrm{SCCM} \mathrm{C}_{4} \mathrm{~F}_{8}$, ICP power $2000 \mathrm{~W}, 20 \mathrm{mTorr}$; etching: $1.75 \mathrm{~s}$ with $275 \mathrm{SCCM} \mathrm{SF}_{6}$, ICP power $2200 \mathrm{~W}$, CCP power increasing linearly from $20 \mathrm{~W}$ at the first cycle to $40 \mathrm{~W}$ in the last cycle, always with a $20 \%$ 

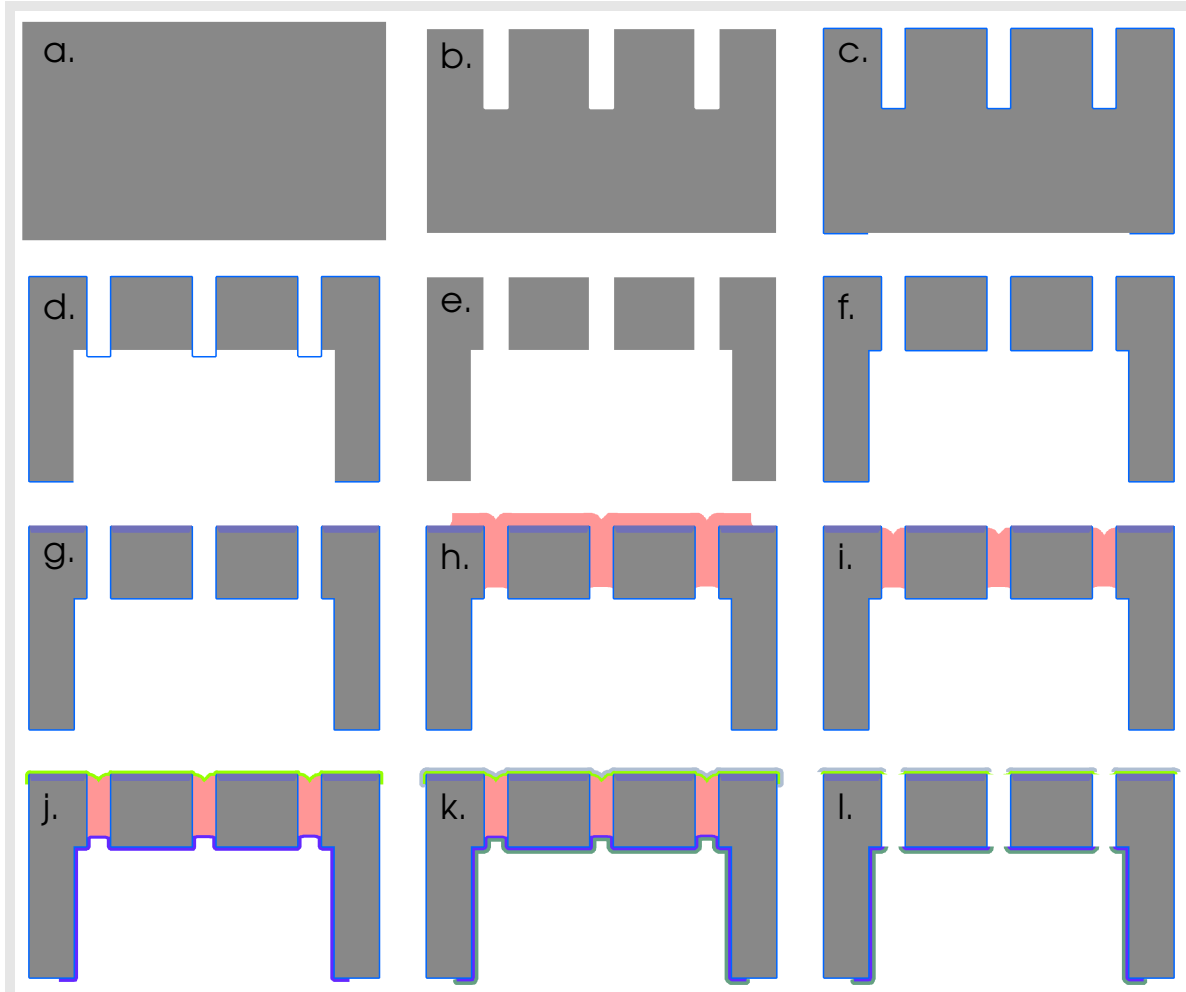

Figure 7.2: Schematic overview of the microfabrication process used for making the membranes. ( $\square \mathrm{Si}, \square \mathrm{Si}_{3} \mathrm{~N}_{4}, \square \mathrm{n}^{+}-\mathrm{Si}$, $\square$ photoresist, $\mathrm{Ti}_{99} \mathrm{Nb}_{1}, \square \mathrm{Cu}, \square \mathrm{Co}-\mathrm{Fe}$ hydroxide, $\left.\square \mathrm{Ni}-\mathrm{Mo}\right)$ Structures resulting from the following steps: (a) start with a silicon wafer, (b) formation of micropores by deep reactive ion etching, (c) conformal coating and back side patterning of silicon nitride to define the membrane area, (d) wet etching of bulk silicon until the bottom of the pores is reached to achieve the desired membrane thickness, (e) opening of the pores by stripping of the silicon nitride, (f) conformal coating with silicon nitride, (g) opening of the front side silicon nitride and phosphorus diffusion to form a highly conductive layer of silicon, (h) photoresist spinning, (i) maskless illumination and development, (j) sputtering of metal electrodes, (k) catalyst electrodeposition and (I) re-opening of pores and metal lift-off by ultrasonication. 
duty cycle, and a pressure of $26 \mathrm{mTorr}$; the wafer was kept at $20^{\circ} \mathrm{C}$ ). The photoresist and fluorocarbons were stripped (TePla 360 barrel etcher; step 1, preheat: $10 \mathrm{~min}, 600 \mathrm{SCCM}$ Ar, $0.6 \mathrm{mbar}, 1000 \mathrm{~W}$; step 2, resist strip: at least $5 \mathrm{~min}$ per wafer, $250 \mathrm{SCCM} \mathrm{O}_{2}, 0.5 \mathrm{mbar}, 800 \mathrm{~W}$; step 3, fluorcarbon strip: $1 \mathrm{~min}, 237 \mathrm{SCCM} \mathrm{O}_{2}, 237 \mathrm{SCCM} \mathrm{CF}_{4}, 0.5 \mathrm{mbar}, 80 \mathrm{~W}$; step 4, residual fluorcarbon removal: $1 \mathrm{~min}, 250 \mathrm{SCCM} \mathrm{O}_{2}, 0.5 \mathrm{mbar}, 800 \mathrm{~W}$ ), followed by RCA-2 cleaning to remove contaminations from the plasma etching machines. After cleaning again in the different nitric acid baths, the silicon oxide hard mask was stripped by etching for 2 min in $50 \%$ $\mathrm{HF}$ solution. To smoothen the inside of the pores, the wafers were oxidized again in the same way as before, but now only for $37 \mathrm{~min}$, to form a silicon oxide layer of approximately $500 \mathrm{~nm}$ thick, which was finally stripped by etching for $1 \mathrm{~min}$ in $50 \% \mathrm{HF}$ solution.

(c) A conformal stoichiometric silicon nitride layer of approximately $50 \mathrm{~nm}$ was grown on the whole wafer by low pressure chemical vapor deposition (LPCVD, $150 \mathrm{mTorr}, 25 \mathrm{SCCM}$ of $\mathrm{SiH}_{2} \mathrm{Cl}_{2}, 250 \mathrm{SCCM}$ of $\mathrm{NH}_{3}$ and $200 \mathrm{SCCM}$ of $\mathrm{N}_{2}$ at $750^{\circ} \mathrm{C}, 48 \mathrm{~min}$ ), to protect the silicon during the etching step that forms the membrane. The unpolished side was patterned using Olin OIR 907-17 positive resist and optical lithography, to define a $2 \mathrm{~cm}$ diameter circle below each hole array, and $20 \mu \mathrm{m}$ wide breaking lines $1 \mathrm{~mm}$ outside each hole array, so that the total size of each device becomes 2.4 by $2.4 \mathrm{~cm}$. This pattern was transferred into the nitride using the same RIE recipe as above for etching silicon oxide, but now only for $150 \mathrm{~s}$. The photoresist was stripped (TePla 360 barrel etcher; step 1, preheat: $10 \mathrm{~min}, 600 \mathrm{SCCM}$ Ar, $0.6 \mathrm{mbar}, 1000 \mathrm{~W}$; step 2, resist strip: at least 5 min per wafer, $250 \mathrm{SCCM} \mathrm{O}_{2}, 0.5 \mathrm{mbar}, 800 \mathrm{~W}$ ), followed by RCA-2 cleaning to remove contaminations from the plasma etching machines.

(d) Any native silicon oxide was removed by etching for $1 \mathrm{~min}$ in $1 \%$ aqueous HF solution, followed by rinsing. The bulk silicon was etched in $25 \%$ aqueous tetramethylammonium hydroxide (TMAH) solution at $70^{\circ} \mathrm{C}$ overnight, such that inside the $2 \mathrm{~cm}$ circles the thickness of the wafer was reduced to approximately $80 \mu \mathrm{m}$. After rinsing and drying, the etch depth was checked using profilometry, and since the etch speed is constant $\left(0.29 \mu \mathrm{m} \mathrm{min}^{-1}\right)$ and uniform over the wafer, the remaining membrane thickness could be controlled accurately to be within 35 to $40 \mu \mathrm{m}$, and the wafers were rinsed and dried. At this point, the pores that were etched from the top have been reached (which was also checked by optical microscopy for every device), such that the silicon nitride layer inside the pores was now exposed from the bot- 
tom. (e) This layer was removed by etching for $25 \mathrm{~min}$ in $50 \% \mathrm{HF}$ solution, followed by rinsing and drying.

(f) A $50 \mathrm{~nm}$ silicon nitride layer was grown as before, this time to protect the inside of the pores from the electrolyte used during measurements $(1 \mathrm{M} \mathrm{NaOH})$, to passivate the silicon surface, and to electrically insulate the bottom electrodes from the silicon. (g) This layer was removed everywhere on the front side by directional RIE etching with the same silicon oxide/nitride etching recipe as before for $150 \mathrm{~s}$, leaving the layer inside the pores and on the back side of the membrane. After cleaning in the acid baths as described for step (a), a phosphorus dopant source layer was deposited by LPCVD (300 nm thick phosphosilicate glass, $350 \mathrm{mTorr}, 675^{\circ} \mathrm{C}, 30 \mathrm{SCCM} \mathrm{N}_{2}$ through the tetraethyl orthosilicate (TEOS) evaporator to form a $50 \mathrm{SCCM}$ TEOS/ $\mathrm{N}_{2}$ mixture, $150 \mathrm{SCCM}$ $\mathrm{O}_{2}, 16.5 \mathrm{SCCM} \mathrm{PH}_{3}$ and $313.5 \mathrm{SCCM}$ argon, $30 \mathrm{~min}$ ), followed by a silicon oxide capping layer ( $100 \mathrm{~nm}$ thick, $350 \mathrm{mTorr}, 675^{\circ} \mathrm{C}, 30 \mathrm{SCCM} \mathrm{N}$ through the TEOS evaporator to form a $50 \mathrm{SCCM}$ TEOS/ $\mathrm{N}_{2}$ mixture, $10 \mathrm{~min}$ ). The phosphorus was made to diffuse into the silicon by heating the samples at $1050^{\circ} \mathrm{C}$ for $15 \mathrm{~min}$ in nitrogen atmosphere, which should lead to a surface doping concentration of above $1 \times 10^{19} \mathrm{~cm}^{-3}$, gradually decreasing to the background phosphorus doping level of around $1 \times 10^{15} \mathrm{~cm}^{-3}$ within $1 \mu^{6}$. Subsequently the phosphosilicate glass was stripped by etching 2 min in $12.5 \% \mathrm{HF}$ in $\mathrm{NH}_{4} \mathrm{~F}$ (1:7 BHF), followed by rinsing and drying.

(h) The wafers were attached with tape onto dummy wafers so that they could be held by the vacuum chucks of a spincoater and an alignment and exposure tool. They were kept on a hot plate at $120^{\circ} \mathrm{C}$ for $5 \mathrm{~min}$, and immediately after that an adhesion layer of hexamethyldisilazane was spincoated for 30 s at 4000 RPM. Olin OIR 907-17 positive resist was poured over all the membranes, and the wafers were spun at 4000 RPM for 30 s so that only a thin layer remained on top. (i) The photoresist was pre-baked on a hot plate at $90^{\circ} \mathrm{C}$ for $90 \mathrm{~s}$ and wafers were exposed to $350-450 \mathrm{~nm}$ UV light with $28.8 \times 10^{6} \mathrm{~J} \mathrm{~m}^{-2}$, which was enough to make the top layer completely soluble, but not the photoresist deep inside the holes. The device wafers were removed from the dummy wafers, baked on a hot plate at $120^{\circ} \mathrm{C}$ for $1 \mathrm{~min}$, developed in Olin OPD 4262 for $1 \mathrm{~min}$, rinsed and dried.

(j) Any native silicon oxide was removed by etching for $1 \mathrm{~min}$ in $1 \%$ aqueous HF solution, followed by rinsing and drying. The electrodes were applied by sputtering metal layers in home-made sputtering ma- 
chines: $\sim 10 \mathrm{~nm} \mathrm{Ti} i_{99} \mathrm{Nb}_{1}$ on the top, anode side, and on the bottom, cathode side, through a shadow mask that covered the edges of the devices, first $\sim 10 \mathrm{~nm}$ titanium as an adhesion layer, and then $\sim 300 \mathrm{~nm}$ copper as the electrode. When nickel was used, or no adhesion layer, the electrode delaminated during the subsequent electrodeposition or device operation.

(k) The wafer was broken along the breaking lines between the membranes, and checked for electrical shorts between the front and back electrode. This was done by measuring the resistance between the cathode and anode with a multimeter, without electrolyte. All devices with a front-to-back resistance lower than $1 \mathrm{k} \Omega$ were discarded. On the remaining devices, electrocatalysts were grown by electrodeposition. For the cathode an electrolyte was prepared consisting of 1.3 M nickel(II) sulfate hexahydrate, $0.5 \mathrm{M}$ boric acid, and $25 \mathrm{mM}$ sodium molybdate in Milli- $Q$ water ${ }^{153}$. The devices were clamped to a $9 \mathrm{ml}$ cell with a side opening of exactly the size of the membrane, which included a platinum mesh as a counter electrode. The copper electrode on the device was contacted with aluminum foil, and a constant current of $63 \mathrm{~mA}\left(20 \mathrm{~mA} \mathrm{~cm}^{-2}\right)$ was applied with the copper electrode as the cathode for $3 \mathrm{~min}$. The cell was emptied and the device removed, and rinsed thoroughly with Milli- $Q$ water. For the anode an electrolyte was prepared consisting of $6 \mathrm{mM}$ cobalt(II) nitrate hexahydrate and $6 \mathrm{mM}$ iron(III) nitrate nonahydrate in Milli- $Q$ water ${ }^{136}$. The electrodeposition was performed in a similar way (also cathodic), except that the anode side of the device was contacted and exposed to the electrolyte, a current of $7.69 \mathrm{~mA}\left(2.45 \mathrm{~mA} \mathrm{~cm}^{-2}\right)$ and a time of $80 \mathrm{~s}$ were used. A brown layer was formed that was rinsed off, leaving a layer that was hardly visible by eye. (I) Finally the photoresist inside the pores was removed by ultrasonication in acetone for $30 \mathrm{~min}$, which also lifted off the metal and catalyst layers that closed the pores on the top and bottom. The devices were rinsed with acetone and dried.

The catalysts were deposited in the same way on flat electrodes. Their catalytic activity was assessed by cyclic voltammetry $(\mathrm{Hg} / \mathrm{HgO}$ reference electrode, $50 \mathrm{mV} \mathrm{s}^{-1}, 5^{\text {th }}$ scan is reported) in $1 \mathrm{M} \mathrm{NaOH}$. 


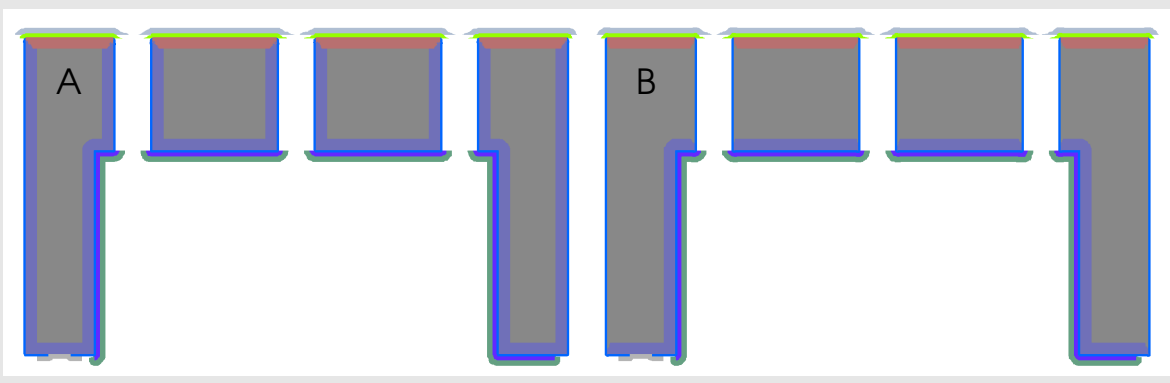

Figure 7.3: Solar-assisted electrolyzer membranes resulting from the two different fabrication routes $\left(\square \mathrm{Si}, \square \mathrm{Si}_{3} \mathrm{~N}_{4}, \square \mathrm{n}^{+}-\mathrm{Si}, \square \mathrm{p}^{+}-\mathrm{Si}\right.$, $\square \mathrm{Ti}_{99} \mathrm{Nb}_{1}$, $\mathrm{Cu}, \mathrm{Co}-\mathrm{Fe}$ hydroxide, Ni-Mo). The difference is in the presence of a highly doped n-type region in the silicon sidewalls for Type A devices.

\subsubsection{Solar-assisted electrolyzer membrane fabrication}

The fabrication of electrolyzer membranes that included a solar cell was similar to the ones without solar cells, with a few changes, described in this subsection. Two approaches were used. The resulting membranes for both approaches are shown in Figure 7.3. For type A, both highly doped regions are formed in one diffusion step, and touch each other near the top op the pores. For type B, the highly doped n-type region is only formed at the bottom of the device, in a separate step, and might undergo limited dopant redistribution during the diffusion step for the p-type region.

\section{Type A}

After step (e), the LPCVD phosphosilicate glass and silicon oxide overlayer were grown as in step (g). These two layers were removed from the top, anode side surface by RIE, as with the silicon oxide etching in step (b), but now for $20 \mathrm{~min}$.

Subsequently, solid source doping was performed. First the wafers were cleaned in the nitric acid baths as described above, including rinsing and drying. They were placed in a furnace with the top side of the wafer facing (but not touching) a boron nitride wafer which served as a source of boron. A layer of boron oxide was grown on the 
wafers at $900^{\circ} \mathrm{C}$ in an atmosphere of 2 standard liters per minute (SLM) $\mathrm{N}_{2}$ and $2 \mathrm{SLM} \mathrm{O}_{2}$ for $15 \mathrm{~min}$, and the atmosphere was changed to only $\mathrm{N}_{2}$ (4SLM) and the temperature of the furnace was further increased to $1050^{\circ} \mathrm{C}$. It remained in that condition for $15 \mathrm{~min}$ to diffuse the boron from the boron oxide and the phosphorus from the phosphosilicate glass into the silicon, and was cooled down again and removed from the furnace. The boron oxide, silicon oxide and phosphosilicate glass were removed by etching for $10 \mathrm{~min}$ in buffered HF solution, followed by thorough rinsing and drying. Any boron silicide that might have formed was oxidized for $15 \mathrm{~min}$ at $800^{\circ} \mathrm{C}$ in oxygen, and the resulting oxide was removed by etching another $10 \mathrm{~min}$ in buffered HF solution, followed by rinsing and drying. In this way a highly doped n-type surface was formed on all silicon surfaces, also inside the pores, but not at the top surface, where a highly doped p-type region was formed, which forms a p/n-junction with the bulk n-type silicon.

Silicon nitride was grown on the doped wafers as in step (f), and patterned as in step ( $\mathrm{g}$ ). Also on the back side the nitride was removed on two patches outside the membranes, to later make contact pads for electrical contact to the n-type bulk silicon. This removing was done using a silicon shadow mask in a home-made parallel plate etching machine (10 mTorr, $25 \mathrm{SCCM} \mathrm{CHF}_{3}, 5 \mathrm{SCCM} \mathrm{O}_{2}, 100 \mathrm{~W}, 1 \mathrm{~min}$ ). The pores were filled with photoresist as in steps (h) and (i). In step (j), directly after sputtering the $\mathrm{Ti}_{99} \mathrm{Nb}_{1}$ on the top of the wafers, $\sim 300 \mathrm{~nm}$ thick aluminum electrodes were sputtered through a shadow mask only on the opened patches on the back. The copper electrodes that were sputtered subsequently were not touching the aluminum contact pads. The wafer breaking, catalyst deposition and pore opening were performed in the same way as the electrolyzer-only membranes.

\section{Type B}

After step (d), silicon nitride was directionally etched from the bottom side with reactive ion etching as before, which removes all silicon nitride from the bottom side of the wafer-thick parts of the device, and from the bottom of the pores. Then, the phosphorus dopant source was deposited, the phosphorus diffused into the silicon, and the source layer removed, as in step ( $g$ ), so that a highly doped n-type region was formed only at the bottom surface of the membrane, the sidewalls around the membrane, and the bottom of the wafer-thick parts 
of the device. Step (e) and ( $f$ ) of the electrolyzer process were performed, as well as the nitride etching of step (g) which removed the nitride selectively from the top surface of the device. Subsequently the solid source doping process was performed as with the Type A solarassisted electrolyzer membranes, so that a highly doped p-type region was formed only on the front side of the membranes.

Further processing was the same as with the Type A membranes: removal of patches of silicon nitride on the bottom side of the devices, pore filling with photoresist, native oxide removal and metal sputtering. However in this case a thicker $(20 \mathrm{~nm}) \mathrm{Ti}_{99} \mathrm{Nb}_{1}$ layer was used. Catalyst deposition and pore opening was not performed for these membranes.

\subsection{3 i-V and stability measurements}

Some devices were not fabricated further than step (f), and the ion transport through these membranes was investigated with external electrodes. The measurement setup is shown schematically in Figure 7.4. The sample was clamped between two glass tubes that are filled with $1 \mathrm{M} \mathrm{NaOH}$, which had an opening with the size of the membrane. In each compartment, far away from the membrane, there is a platinum wire electrode, and a fixed current is forced between these electrodes. Also in each compartment there was a capillary, filled with the same electrolyte and containing a $\mathrm{Hg} / \mathrm{HgO}$ reference electrode, with its tip close to the membrane surface. The current between the platinum electrodes was now ramped, and the voltage drop between the reference electrodes was recorded. The slope of the current-voltage relation is the resistance of the membrane plus the resistance of the electrolyte layers between the membrane and the capillary tips. The experiment was also performed without a membrane, so that the resistance of these electrolyte layers can be determined and subtracted from the other measurements. The remaining resistance is the resistance of the membrane itself.

The fully fabricated devices were clamped between two PTFE cells that have an opening with the size of the membrane on the side. In this way there was an electrolyte compartment of $\sim 10 \mathrm{ml}$ on both sides of the membrane. Between the device and each cell there was a Viton sealing pad. On this seal electrodes are made using copper tape, 


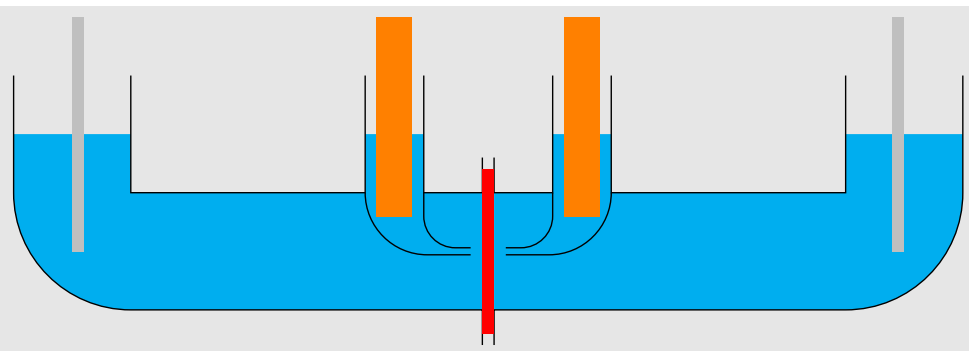

Figure 7.4: Schematic representation of the measurement setup used for conductivity measurements of the membranes with external electrodes. The sample (red) is clamped between two tubes that are filled with electrolyte (blue). Each compartment has a distant platinum wire electrode (gray), and a capillary with its tip close to the membrane and a reference electrode (orange) inside.

such that when the membrane was clamped, the copper strips contact the anode side on two locations and the cathode electrode on one location. Both compartments were filled with $1 \mathrm{M} \mathrm{NaOH}$ solution in Milli-Q water, and a current of $31 \mathrm{~mA}\left(10 \mathrm{~mA} \mathrm{~cm}^{-2}\right)$ was applied until the voltage needed to sustain that current stayed within $50 \mathrm{mV}$ for at least $30 \mathrm{~min}$ (usually this was achieved in one to two hours) to make sure that the catalysts were in a more or less stable situation. The voltage was swept from 0 to $3.5 \mathrm{~V}$ with $\sim 200 \mathrm{mV} \mathrm{min}^{-1}$, where the voltage on the anode side was provided by one copper strip and measured with the other to overcome any contact resistances that were experienced on that side. The current was measured for each step of $100 \mathrm{mV}$. For most devices still a minor current was measured below $1.23 \mathrm{~V}$, which cannot be due to water splitting and is therefore seen as a leakage current. This leakage current is assumed to increase linearly with applied voltage, and this predicted leakage current is therefore also subtracted from the currents measured above 1.23 V. All shown results are based on these corrected currents.

To check the stability of a device, it was measured in the same PTFE cell with the same connections and electrolyte. A constant current of $31 \mathrm{~mA}\left(10 \mathrm{~mA} \mathrm{~cm}^{-2}\right)$ was applied and the voltage recorded every minute for $24 \mathrm{~h}$. 


\subsubsection{Solar-assisted i-V measurements}

To assess the performance of the devices where the silicon is also used as a photovoltaic material, they were measured in a similar way as the electrolyzer membranes. One cell had a glass window with the same size as the membrane, and between the glass and the membrane there was $2.6 \mathrm{~cm}$ of electrolyte. Through the glass and electrolyte, the membrane was illuminated from the top, anode side with a simulated AM $1.5 \mathrm{G}$ solar spectrum with $1000 \mathrm{~W} \mathrm{~m}^{-2}$ in the same way as described in Section 5.3.7. The voltage was now applied between the copper and aluminum electrodes, and the current recorded. The same devices were also measured as an electrolyzer-only device, by measuring the current-voltage relation between the front side $\mathrm{Ti}_{99} \mathrm{Nb}_{1}$ electrode and the back side copper electrode. Also for these measurements, the electrolysis was first run for one to two hours to reach a stable situation.

The performance of the silicon $\mathrm{p} / \mathrm{n}$ junction with the $\mathrm{Ti}_{99} \mathrm{Nb}_{1}$ protection layer and catalyst on top was also tested separately as a solar cell and as a photoanode. For this purpose the same silicon substrates were used as for making the membranes, but they were left flat: no pores and membranes were etched. $\mathrm{p}^{++}$and $\mathrm{n}^{++}$doped areas were made on the front and back side, respectively. The same $\mathrm{Ti}_{99} \mathrm{Nb}_{1}$ protection layer was sputtered on the front, and a thicker layer on the back as a back side contact. The cobalt-iron hydroxide was grown on the protection layer. The samples were put in the same configuration as for testing the catalysts, and were illuminated from the front with a solar simulator in the same way as the membranes. The currentvoltage relation was measured between the front and back side of the sample (photovoltaic behavior) and between the back side electrode and the reference electrode (photoanode behavior).

\subsubsection{Gas separation measurements}

The setup for detecting hydrogen crossover for the electrolyzer membranes is shown schematically in Figure 7.5. The sample was clamped between two PTFE cells as for the i- $V$ measurements, but one cell was closed off from the environment, and had a fixed $10 \mathrm{ml} \mathrm{min}^{-1}$ argon inflow, and the outflowing gas mixture was analyzed by gas chromatography (GC). The 'CompactGC Interscience' GC was equipped with a 


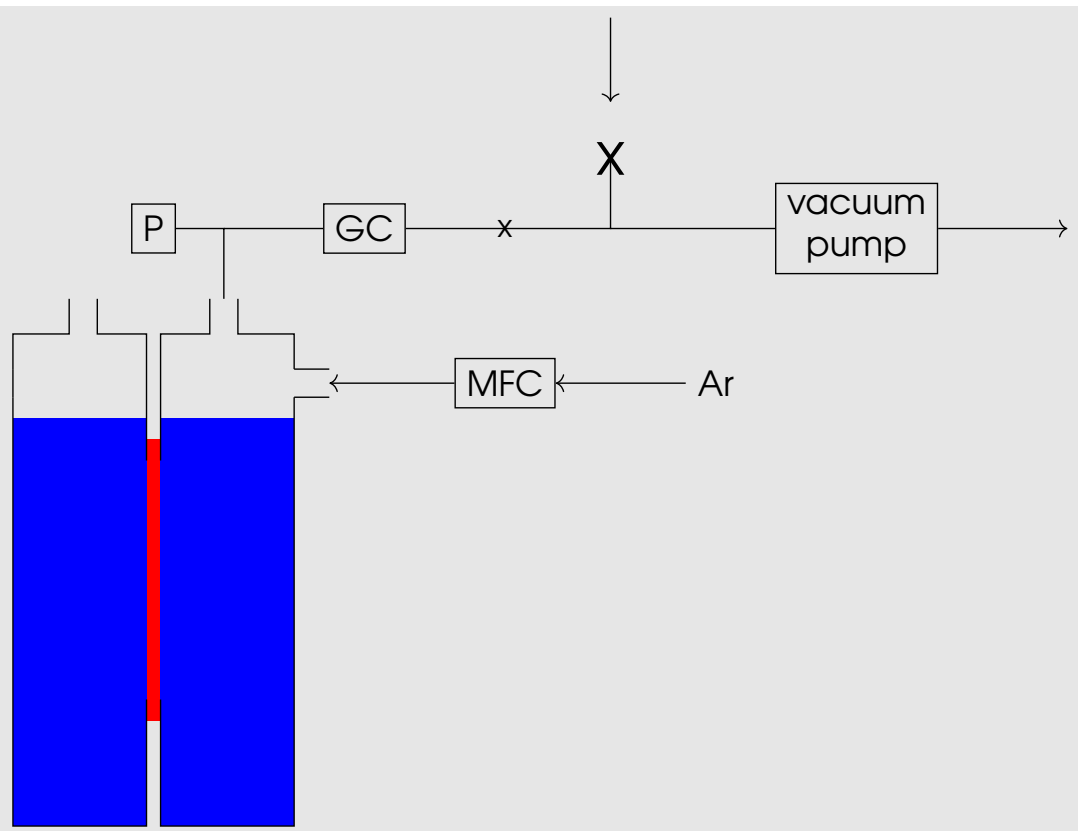

Figure 7.5: Schematic representation of the measurement setup used for ion transport and gas crossover characterisation of the membranes. The sample (red), which has a membrane in the middle, is clamped between two fluid compartments with side openings allowing the electrolyte (blue) to access the membrane. The left cell is open to the atmosphere, the right cell is closed and has an inflow of carrier gas (Ar) controlled with a mass flow controller (MFC). The gas pressure in the cell is measured at the outlet $(P)$, which is connected to the gas chromatograph (GC). Pressure buildup in the right cell is prevented by connecting a vacuum pump behind the GC, and the pressure in the cell is regulated manually by adjusting two valves ( $x$ and $X$ ).

Parabond $Q$ column ( $10 \mathrm{~m}$ ) and a thermal conductivity detector to determine the hydrogen and oxygen concentrations. From the outflow, a $50 \mathrm{Hl}$ sample was taken every $130 \mathrm{~s}$ and the composition analyzed. With the settings used, the GC was mainly sensitive to hydrogen, but larger concentrations of oxygen could also be measured.

To ensure a stable situation, an electrolyzing current of $31.4 \mathrm{~mA}$ (10 
$\mathrm{mA} \mathrm{cm}^{-2}$ ) was applied for at least $4 \mathrm{~h}$, and the gas concentrations of the last $2 \mathrm{~h}$ were averaged to find the average gas production and crossover rate. The gas composition of the anode compartment was measured for several devices to assess the hydrogen crossover rate. The cathode compartment was also analyzed for one device but because of the limited sensitivity of the GC to oxygen, no oxygen could be reliably detected.

\subsection{Results and discussion}

\subsubsection{Fabrication results}

Figure 7.6 shows cross-sectional views of the perforated membrane. Figure 7.6(a) shows the size and shape of a pore. For the pores in this picture, the oxidation and oxide etching at the end of step (b) was not applied, to show the intrinsic roughness caused by the Bosch etching process that was used to form the pores. Figure 7.6(d) shows the $\mathrm{Ti}_{99} \mathrm{Nb}_{1}$ layer on the top, anode side of the membrane, which was patterned by lift-off of the photoresist inside the pores, overlapping partially with the silicon nitride layer inside the pores, which was patterned by directional etching from the top. In this way the silicon is completely protected from the electrolyte. Figure 7.6(b) shows the structure of the electrodeposited oxygen evolution catalyst at the edge of a pore. Figure 7.6(c) shows the bottom, cathode side of the pore, showing that the copper electrode and hydrogen evolution catalyst extend somewhat into the pore, indicating that the photoresist did not completely reach the bottom of the pore in step (h). Additionally, close to the bottom the pore diameter is reduced to about $1.5 \mu \mathrm{m}$ (Figure 7.6(c)), while the maximum diameter of the pores is around $3.3 \mu \mathrm{m}$ close to the top (Figure 7.6(a)).

Assuming a linear decrease of the pore diameter from $3.3 \mu \mathrm{m}$ at the top to $1.5 \mu \mathrm{m}$ at the bottom, an effective porosity can be calculated for the sake of ionic conductivity, compared to a straight pore with the designed $3.0 \mu \mathrm{m}$ diameter along the whole length. The resistance of a straight pore would be

$$
R_{\mathrm{s}}=\rho \frac{4 l}{\pi d^{2}},
$$



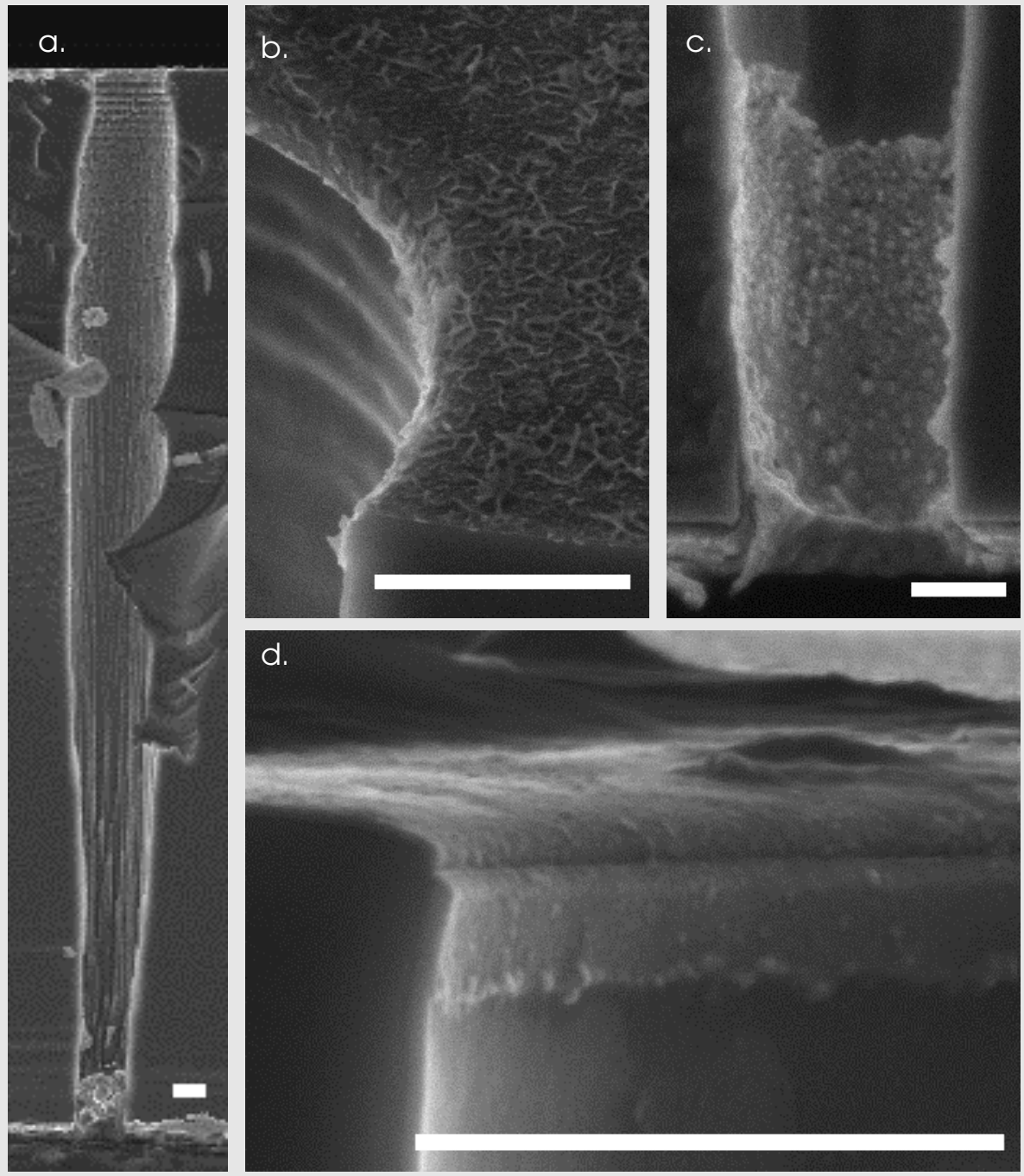

Figure 7.6: Cross-sectional SEM pictures of the silicon membranes with pores. (a) Full pore made without the smoothing step, (b) edge of a pore at the top (anode) side, with the cobalt-iron hydroxide catalyst, (c) a pore on the bottom (cathode) side, with nickel-molybdenum catalyst and (d) edge of a pore on the anode side, without catalyst deposition. Scale bars are $1 \mu \mathrm{m}$. 
where $\rho$ is the resistivity of the electrolyte, $l$ is the length of the pore and $d$ the diameter. For a tapered pore, this equation has to be integrated along the length of the pore to find the resistance of a tapered pore:

$$
R_{\mathrm{t}}=\rho \int_{0}^{l} \frac{4 \mathrm{~d} x}{\pi d(x)^{2}}
$$

where $x$ would be the coordinate along the length of the pore and

$$
d(x)=3.3+x \frac{1.5-3.3}{l}=3.3-x \frac{1.8}{l} .
$$

Substituting (7.3) into (7.2) gives

$$
\begin{aligned}
R_{\mathrm{t}} & =\rho \int_{0}^{l} \frac{4 \mathrm{~d} x}{\pi\left(3.3-x \frac{1.8}{l}\right)^{2}}=\left[\frac{4 l}{1.8 \pi\left(3.3-x \frac{1.8}{l}\right)}\right]_{0}^{l} \\
& =\frac{4 l}{\pi 1.8}\left(\frac{1}{3.3-1.8}-\frac{1}{3.3}\right)=\frac{4 l}{\pi 2.2^{2}} .
\end{aligned}
$$

Comparing with (7.1) this shows that such a pore would have the same resistance as a straight $2.2 \mu \mathrm{m}$ diameter pore, and that therefore the porosity, which scales with $d^{2}$, is effectively only $2.2^{2} / 3.0^{2}=55 \%$ of the designed value.

\subsubsection{Catalyst and photoanode performance}

Figure 7.7 shows the improved hydrogen evolution on the cathode side if the nickel-molybdenum alloy catalyst is deposited. At $10 \mathrm{~mA} \mathrm{~cm}^{-2}$, an overpotential below $200 \mathrm{mV}$ was achieved. Figure 7.8 shows that for the electrolyzer anode there was no significant oxygen evolution if no catalyst was applied, while with the cobalt-iron hydroxide catalyst the overpotential (with respect to $1.23 \mathrm{~V}$ vs RHE) was around $500 \mathrm{mV}$ at 10 $\mathrm{mA} \mathrm{cm}^{-2}$. When the silicon below the metal protection layer was used as a solar cell, this overpotential was reduced by $\sim 450 \mathrm{mV}$ to $\sim 50 \mathrm{mV}$ at $10 \mathrm{~mA} \mathrm{~cm}^{-2}$, while at $1.23 \mathrm{~V}$ vs RHE the photocurrent was $7.3 \mathrm{~mA} \mathrm{~cm}^{-2}$. Furthermore the current was limited at the maximum photocurrent of the photovoltaic cell, around $15 \mathrm{~mA} \mathrm{~cm}^{-2}$.

The photovoltaic cells were also characterized separately, and the results are shown in Figure 7.9. This Figure shows that the presence of 


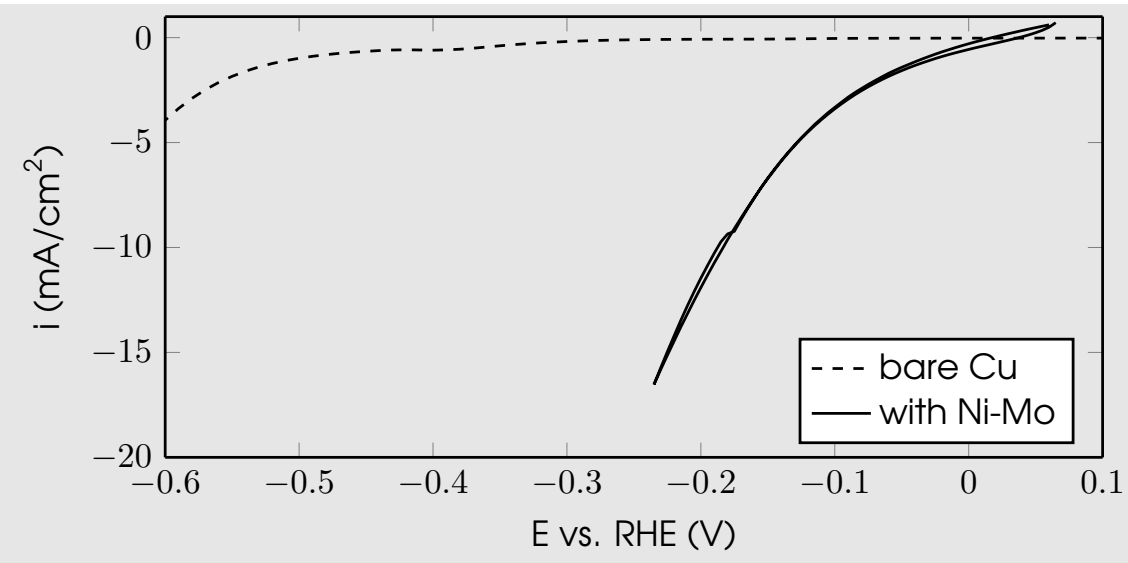

Figure 7.7: Current density versus potential of a bare copper electrode and one on which the Ni-Mo hydrogen evolution catalyst has been deposited.

a catalyst layer reduced the photocurrent, which can be explained by parasitic light absorption by the catalyst. Furthermore at $10 \mathrm{~mA} \mathrm{~cm}^{-2}$ the photovoltaic cell should be able to reduce the voltage required for electrolysis by at least $400 \mathrm{mV}$, which is in good agreement with the reduction of overpotential observed in the photoanode measurements on the same device.

\subsection{3 i-V and stability measurements}

Figure 7.10 shows the measurement results for one of the fully fabricated electrolyzer membranes. When it started electrolyzing when more than $\sim 1.7 \mathrm{~V}$ was applied between the top and bottom side electrode, the current density increase with voltage quickly became linear, instead of the exponential behavior that would be expected from reaction limited behavior according to the Tafel and Butler-Volmer equations. The constant slope indicates that the device was limited by resistive behavior in this regime. This is attributed to the ionic conduction in the electrolyte. The slope in this part of the graph, which equals the conductivity, was determined for each device. From the conductivities of the fully fabricated membranes and the membranes that were 


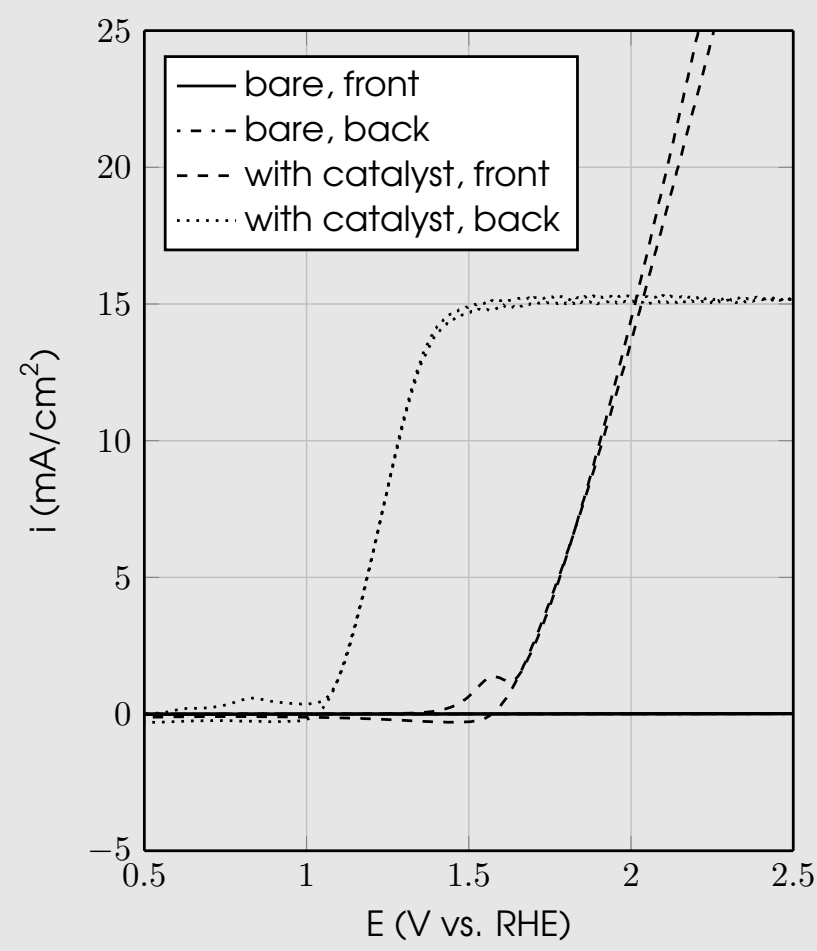

Figure 7.8: Current density versus potential of a bare 'solar cell' used as an anode (contacted from the front) and photoanode (contacted from the back, both 'bare' lines overlap), and for one on which the cobalt-iron hydroxide electrocatalyst has been deposited.

measured with external electrodes, the voltage drop over the membrane at a current density of $10 \mathrm{~mA} \mathrm{~cm}^{-2}$ was calculated, and these values are shown in Figure 7.11.

Both series of measurements were fitted with a $V(p)=i c / p$ curve (potential drop $V$ is the product of current density $i$ and constant $c$ divided the porosity $p$, where $p=0.01$ for a $1 \%$ porous device), which appears to be a good approximation of the trend. This indicates that the conductivity of the membrane increases linearly with the porosity, confirming again that the determined slopes are caused by the resistance of the pores. For the outlier at $2 \%$ porosity, with a solid marker, 


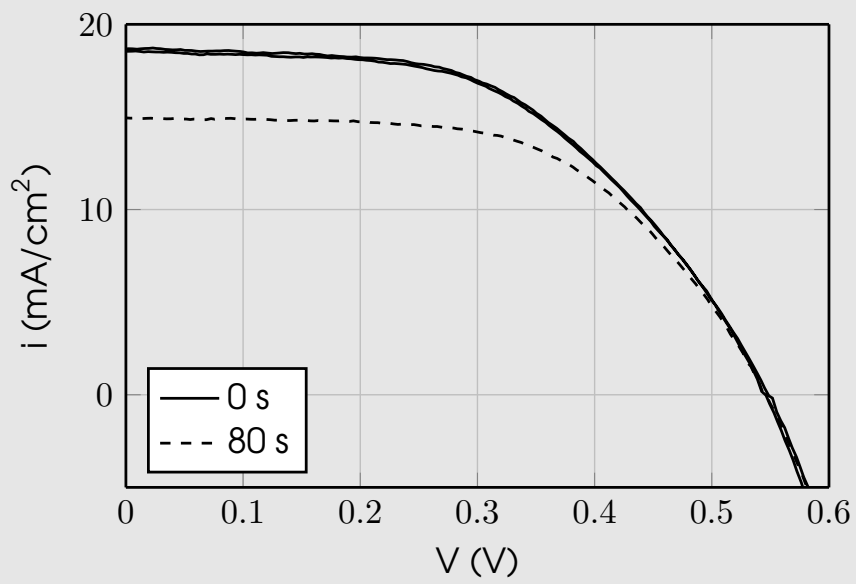

Figure 7.9: Current density versus applied potential when the photoanode used in the membrane was measured as a solar cell, with the cobalt-iron hydroxide electrocatalyst deposited for $80 \mathrm{~s}$ or without catalyst (0s).

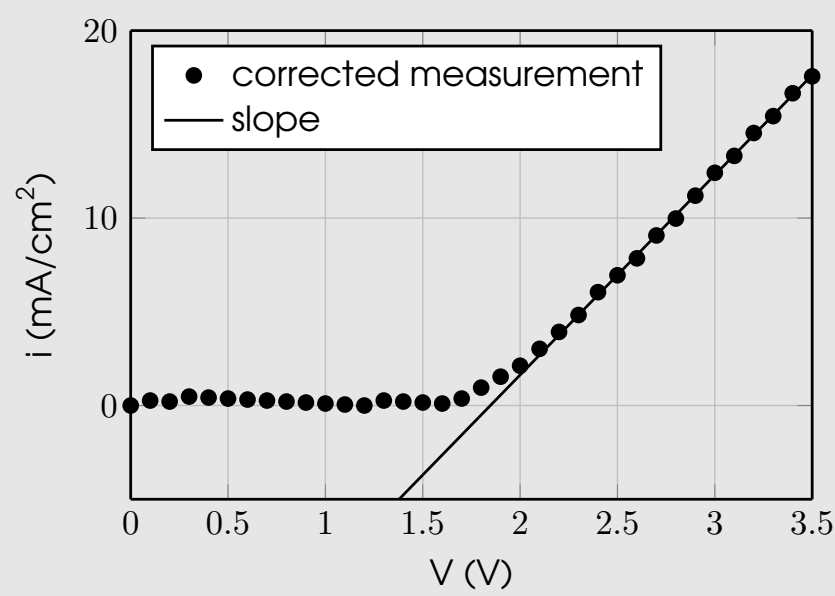

Figure 7.10: Example membrane measurement which was used to find the ion transport overpotential (after correcting for a small leakage current), for a $0.031 \%$ porous membrane. 


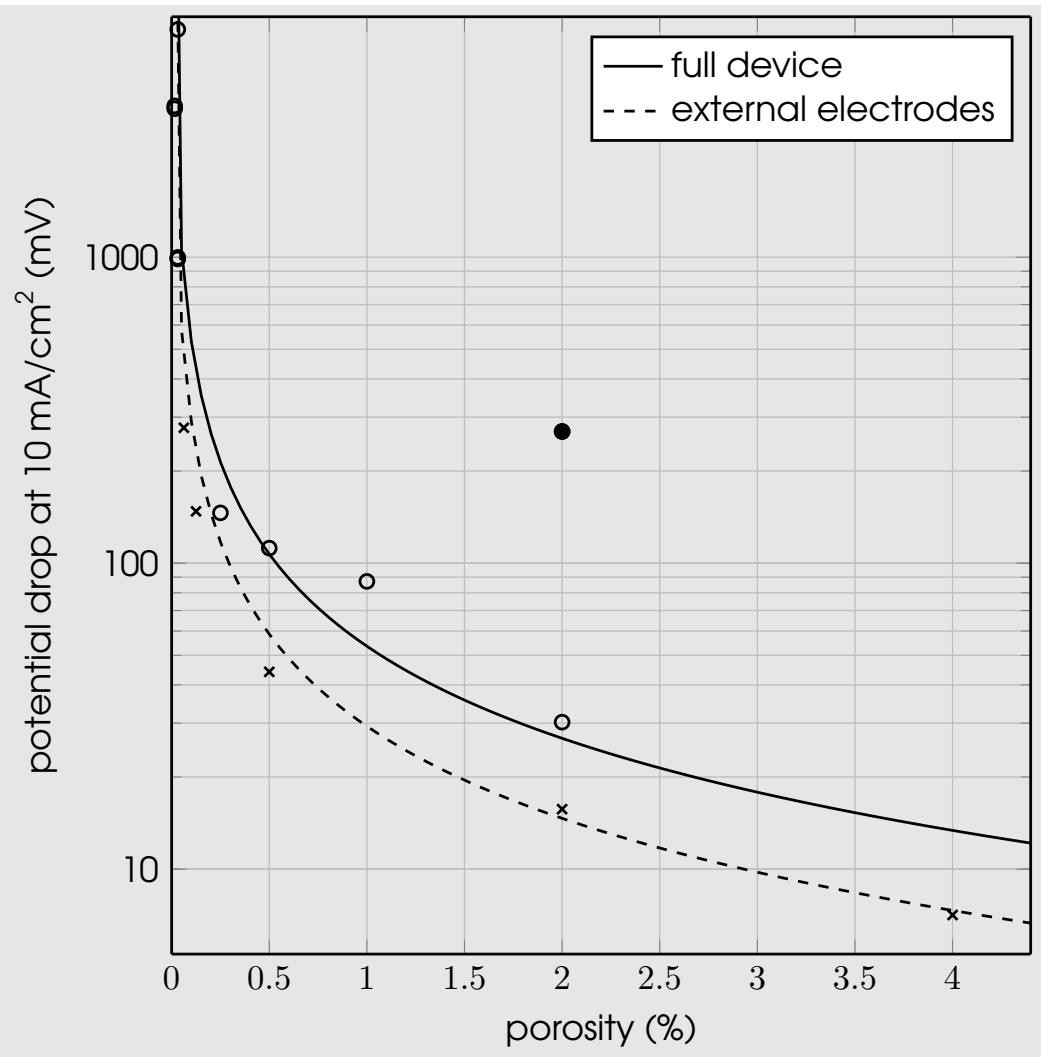

Figure 7.1 1: Ion conduction overpotentials at $10 \mathrm{~mA} \mathrm{~cm}^{-2}$ of membranes with various porosities. Circles are values based on fitted slopes from full device measurements, crosses are values based on devices with external electrodes. Lines indicate the fitted $V(p)=i c / p$ trends for both cases. 
part of the copper electrode had delaminated and the determined conductivity may in this case have been limited by the poor electrical conduction between the location where the device was contacted electrically, and the copper electrode on the surface of the membrane.

$c$ is $2.9 \times 10^{-6} \Omega \mathrm{m}^{2}$ for the fit of the measurements with external electrodes and $5.4 \times 10^{-6} \Omega \mathrm{m}^{2}$ for the fully fabricated devices. The findings of Bosserez et al. ${ }^{17}$ show that ion transport along the surface of the membrane has a negligible contribution to the ion transport resistance at these length scales. Therefore the resistance is fully attributed to the pores, and with an electrolyte conductivity of $20.6 \mathrm{~S} \mathrm{~m}^{-119}$ and a pore length of $40 \mu \mathrm{m}$, a value of $c=1.9 \times 10^{-6} \Omega \mathrm{m}^{2}$ would be expected. This indicates that indeed the porosity was lower than designed, especially for the fully fabricated devices. This can partially be attributed to the reduced pore size because of electrode metal and catalyst deposition inside the pore and the tapering resulting from the Bosch etching (see Figure 7.6(a,c)) and Section 7.3.1.

For devices with porosities down to $0.25 \%$, the ion transport overpotential was smaller than the lowest reaction overpotential (at the cathode). This shows that this type of device can make the ion transport overpotential a negligible loss while keeping almost the full surface area of the device available for light absorption, thereby effectively bypassing the trade-off between ion transport and light absorption.

The Type A solar-assisted electrolyzer membranes had a large but irreproducible leakage current between the front and back side electrode when they were measured without electrolyte. This was probably caused by the direct contact between the highly doped $p$ and n-type regions: a leaky junction may have been formed at this interface. Therefore measurements in electrolyte could not be performed. The type B membranes were only measured as solar cells, without electrolyte. The results are shown in Figure 7.12. They include a measurement on a reference device that contained no membrane and no pores, but the same dopants and metal overlayer (labeled as $0 \%$ ), which unfortunately also had a large leakage current between front and back. The closed circuit photocurrent of this device can however still be compared with that of the other devices, since when $0 \mathrm{~V}$ is applied this leakage current should be absent. In general, photocurrents were decreasing significantly with increasing porosity, from $11 \%$ for the $0.031 \%$ porous devices to $34 \%$ for the $4 \%$ porous devices, as compared 


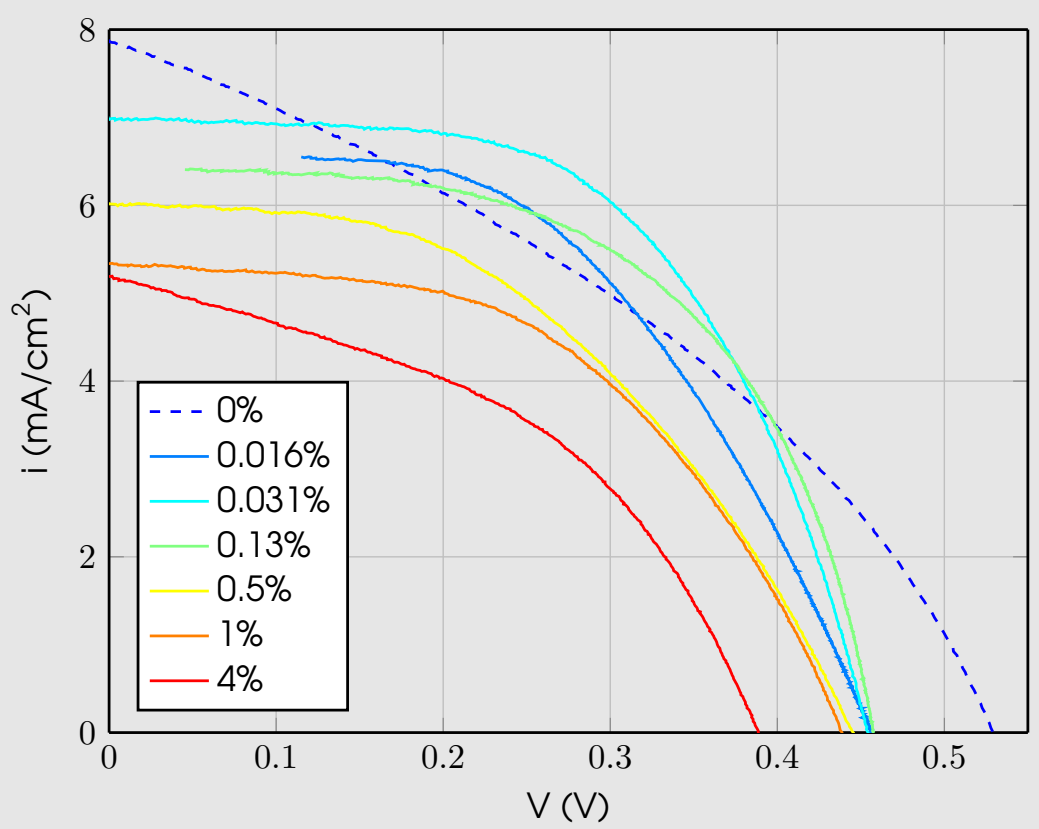

Figure 7.12: Solar cell characteristics of Type B solar assisted electrolyzer membranes with different porosities as indicated in the legend, measured without electrolyte. The ' $0 \%$ ' device is not a membrane but a flat silicon solar cell covered with the same $\mathrm{Ti}_{99} \mathrm{Nb}_{1}$ protection layer.

to the flat reference device. This was probably caused by increased recombination because of the defects and additional surface area introduced by the pores. These devices were not tested for electrolysis because a current density of $10 \mathrm{~mA} \mathrm{~cm}^{-2}$ could not be reached, probably because of parasitic light absorption by the thicker $\mathrm{Ti}_{99} \mathrm{Nb}_{1}$ layer.

Figure 7.13 shows the voltage over a $0.5 \%$ porous device when a constant current was applied for $24 \mathrm{~h}$. After this, the sample was taken out, and the $\mathrm{Ti}_{99} \mathrm{Nb}_{1}$ layer was found to be oxidized, which explains the slow increase in potential that was necessary to keep the current constant. This might be prevented by coating a transparent conductive oxide like FTO on top of the $\mathrm{Ti}_{99} \mathrm{Nb}_{1}$ layer. Other devices failed sooner, and with these devices delamination of the copper cathode was al- 


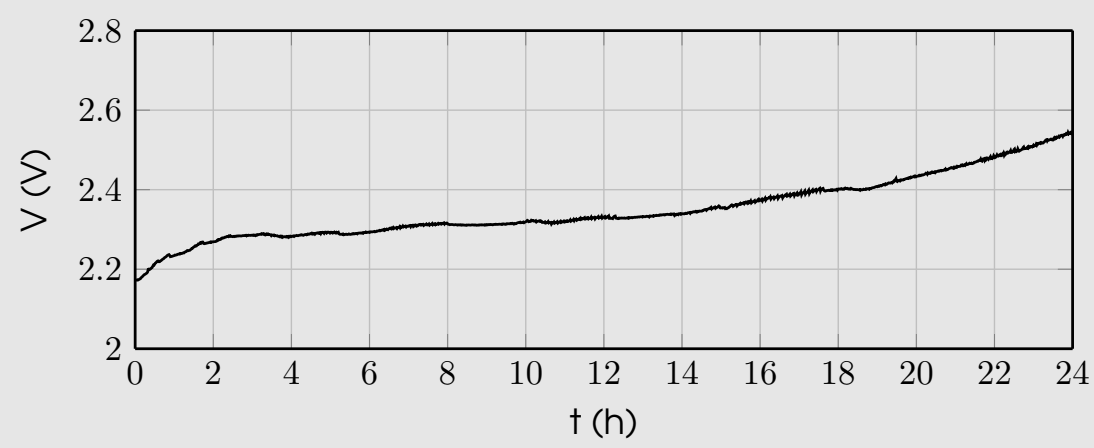

Figure 7.13: Solar cell characteristics of Type B solar assisted electrolyzer membranes, measured without electrolyte.

ways observed. This may have been caused by a thin photoresist layer formed during the pore filling procedure, which dissolves in the electrolyte, causing lift-off of the electrode. This might be prevented by applying a short oxygen plasma ashing step before the metal deposition.

\subsubsection{Gas separation}

Table 7.1 shows the gas composition measurement results. For the gas produced in a certain compartment (oxygen for the anode and hydrogen for the cathode), the production is compared to the gas production expected based on the corrected applied current. The current correction was done by subtracting a leakage current that was predicted based on the $i$ - $V$ measurements. In the anode compartment the measured concentration of hydrogen gas (which arises from leakage through the pores) is compared to the measured concentration of the oxygen gas that is produced in this compartment. In the cathode compartment similar measurements were not possible, because the GC was not sensitive enough to detect small amounts of oxygen in hydrogen.

As can be seen in Table 7.1, the hydrogen crossover stayed well below the flammability limit, and is expected to be caused by diffusion of dissolved hydrogen through the electrolyte inside the pores. Since the 


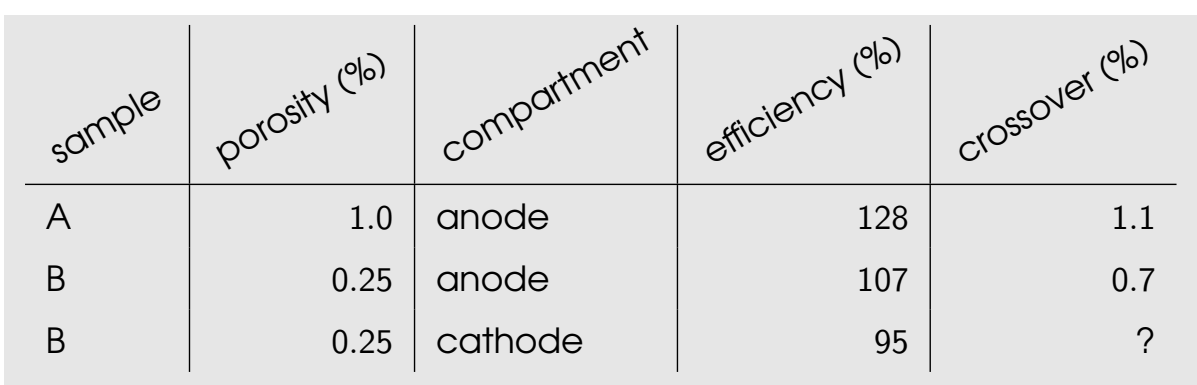

Table 7.1: Gas composition measurements of anode and cathode compartments for two devices with different porosities. The efficiency is the ratio between the produced gas in that compartment and the amount of gas expected from the applied current (corrected for the leakage current). The crossover is the ratio between the measured hydrogen and oxygen in that compartment.

diffusion rate of oxygen in water is lower than that of hydrogen, oxygen crossover is also expected to stay below the flammability limit. For sample $B$, the amount of oxygen and hydrogen produced was close to the expected value based on the (corrected) applied current. Deviations could be the result of the inaccuracy of the gas measurement setup. For sample A, the oxygen production seems to be much higher than the production based on the corrected current. Perhaps the the leakage current was overestimated in this case (at about $32 \%$ of the total current), or there may have been an air leak into the setup.

\subsection{Conclusion}

A solar water splitting device concept has been discussed that should allow safe and efficient unassisted water splitting using only earth-abundant materials. In this device concept a thin (tens of micrometers) stack of light-absorbing semiconductors is perforated with a regular array of well defined micropores to allow ion transport between the anode and cathode. Devices have been made with which the ion transport and gas separation properties of this device concept could be tested. It was shown that even when only $0.25 \%$ of the device surface area was used for ion transport, the ion transport overpotential could 
be kept below $200 \mathrm{mV}$ at a current density of $10 \mathrm{~mA} \mathrm{~cm}^{-2}$. The remaining surface area is still available for light absorption. At the same time, without using an ion selective separator membrane, gas crossover could be kept at around $1 \%$. These devices have shown stable operation for hours, but require further testing and engineering for achieving stability on longer time scales.

Only preliminary investigations into the solar energy conversion performance of such devices have been conducted. The silicon membrane was successfully converted to a solar cell which in theory can provide part of the voltage needed for water splitting. The pores in the membrane reduced the photocurrents of these cells more strongly than would be expected on the basis of the surface area reduction that they cause.

The measurements show the promise of the presented device concept for bypassing trade-offs between ion transport, light absorption and gas separation. However the full device concept has not yet been tested. To be able to do that, the main challenge is to add additional layers on the silicon so that the layer stack can provide the voltage and current necessary for water splitting at a reasonable rate. Chapter 6 has discussed the combination of crystalline silicon with hematite. The voltage provided by this stack is still too low, and the currents are far below what could theoretically be expected from hematite. Other semiconductors might be used, like bismuth vanadate ${ }^{154}$, or a triple or quadruple cell with microcrystaline and/or amorphous silicon 123,155, to achieve the voltage and current required. Adaption of the fabrication scheme and used materials may be required because of compatibility issues of these semiconductors and their synthesis routes with the fabrication methods that have been used in this work.

Furthermore some of the fabrication techniques used, and especially the deep reactive ion etching used for forming the pores, may be too expensive for producing large surfaces of solar water splitting devices. This can perhaps be replaced by metal-assisted chemical etching ${ }^{156}$, which can more easily be applied over large surface areas, even though it probably produces smaller and less uniform pores. 


\section{Chapter 8}

\section{Outlook: integrated stand-alone solar water splitting}

So far, this thesis has discussed several exploratory studies on the merits of microstructured and fully integrated solar-to-hydrogen devices. Chapter 9 will give some considerations whether full integration is indeed preferable from a societal perspective. In the present Chapter the desirability of fully integrated devices is a premise. Based on this assumption, it is discussed what technological advances would be necessary or expected to achieve a fully integrated device that produces separate hydrogen and oxygen gas streams with only water and solar irradiation as inputs, at a rate where the sales of the produced hydrogen justify the fabrication and operational costs of the device as much as possible. That means that the device will have to be as efficient as possible, should hardly degrade over time, and the fabrication methods and materials used should not prohibit the fabrication of large surface areas of that device. 


\subsection{Architectures}

Any photoelectrochemical device should have a closed loop of charge transport, and there are several elements in this loop, as shown in Figure 8. 1. If the loop would not be closed there would at some points be infinite charge buildup. The total stack of semiconductors that provide the water splitting voltage and current is here arbitrarily split up into 'cathodic absorbers' and 'anodic absorbers', but could in fact consist of only one absorber material, the two stacked subcells of a tandem device, or an arbitrary grouping of subcells if there are more than two, as for example in triple or quadruple cells. The 'cathode' could simply be the interface between the 'cathodic absorbers' and the catholyte, one or several layers (catalyst, conductive or protecting) on the surface of the 'cathodic absorbers', or an electrode physically separated from the 'cathodic absorbers', only connected by an electrically conductive wire. This holds mutatis mutandis also for the 'anode'.

Now in principle, one can think of many configurations in which the loop is closed in this way. However what has to be considered is that the light has to reach all the semiconductor absorbers. In most configurations this would mean that the light will also pass through other elements of the circle. This could lead to parasitic light absorption. Furthermore it has to be taken into account that if two or more absorber subcells are used (tandem, triple, quadruple, ...), the light would be used in the most optimal way if it passes first through the absorber with the highest band gap and subsequently through materials with ever lower band gaps 7,138 .

For example for the device concept presented in Chapter 7 and the 'wireless' design of Reece et al. ${ }^{15}$, the light passes through the anolyte and 'anode' before it reaches the 'anodic absorbers' and then the 'cathodic absorbers' (Type A), while for their 'wired' design the light also had to pass through their 'cathode' (since no membrane was used in this case, the anolyte and catholyte cannot be discerned) (Type B). Also conceivable is a configuration where the light first passes through a transparent conductive 'superstrate' on which the 'anodic absorbers' are deposited, and then subsequently through the anode, anolyte, membrane, catholyte, and cathode, before it reaches the 'cathode absorbers' (a configuration in which the photocathode presented in Chapter 5 would be useful) (Type C). A configuration in which 


\subsection{ARCHITECTURES}

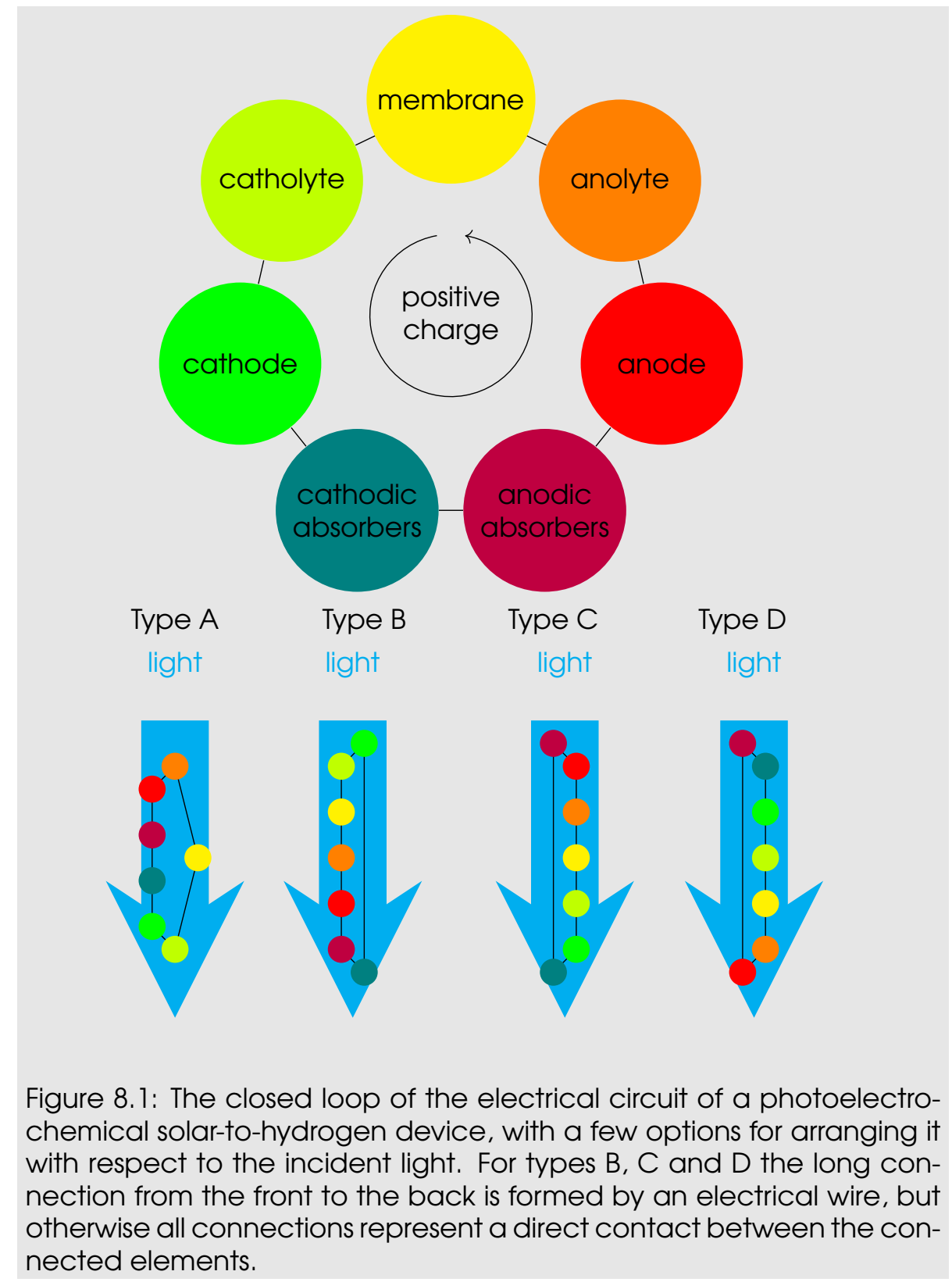


one would have to worry the least about parasitic light absorption would be one where the whole electrolyzer (cathode, catholyte, membrane, anolyte, anode) is integrated on the back side of a photovoltaic cell which produces the full voltage needed for water splitting, which would only need electrical wiring between the top side of the photovoltaic cell and the bottom electrode of the electrolyzer (Type D). These are only examples and many other types and variations on these are conceivable, but these are used to illustrate several considerations below.

The integrated configuration Type D would not have clear advantages over a non-integrated design (separate photovoltaic cell and electrolyzer), except if the absorber with the lowest band gap would benefit from a semiconductor-liquid interface. Instead, most photoelectrode materials considered for this application, like $\alpha-\mathrm{Fe}_{2} \mathrm{O}_{3}, \mathrm{WO}_{3}$, $\mathrm{BiVO}_{4}$ and $\mathrm{Cu}_{2} \mathrm{O}$, have a band gap of over $2 \mathrm{eV}$ and are therefore more suitable as the top semiconductor. That would require a Type A, B or C configuration (where Type $A$ and $C$ could also have a semiconductorliquid interface at the cathode), and therefore would always suffer some parasitic light absorption by the 'anode' and anolyte. Type C could additionally suffer from light absorption by the membrane, catholyte and 'cathode'. Therefore, the choice of absorber materials will influence which configuration would be most optimal, and this choice is discussed in the following Section, 8.2.

\subsection{Absorber stack}

In Section 2.1.2 it was argued that a tandem light absorber, with the bottom band gap around $1.0 \mathrm{eV}$ and the top band gap around 1.6-1.8 $\mathrm{eV}$ would be most ideal for producing the voltage and current needed for water splitting. However given the poor performance of all earthabundant semiconductors with a higher band gap, probably the high band gap should be even higher in practice. In Chapter 6 an integrated tandem of crystalline silicon ( $1.1 \mathrm{eV}$, buried junction) and hematite $(2.1 \mathrm{eV}$, semiconductor-liquid interface) was investigated. However when this tandem was used as a photoanode, it only started to show positive photocurrents above $0.4-0.7 \mathrm{~V}$ vs RHE. Since such an analysis does not include the extra overpotentials produced by the cathode 
kinetics and ion transport, probably another $0.5-0.9 \mathrm{~V}$ has to be produced somehow. As was argued in Section 6.3, some, or maybe all of this improvement could be provided by further engineering of the silicon bottom cell and the electrical interconnect layer, and by improving the electrical properties of the hematite and the catalytic properties of its surface. Indeed, onset potentials as low as $0.58 \mathrm{~V}$ vs RHE have been reported for hematite ${ }^{157}$, and the highest reported open circuit voltage for a crystalline silicon cell is around $0.74 \mathrm{~V}^{100}$, which is close to the theoretical maximum. Given the shown detrimental effect of the interface layer on the solar cell performance when it is subjected to the hematite synthesis conditions, as shown in Section 6.2.1, achieving a stand-alone water splitting device with such an integrated tandem will still be a challenge. Also the silicon solar cell may deteriorate under these synthesis conditions. Furthermore hematite will need measures like a structured substrate, plasmonic particles and reflecting layer to approach its theoretical maximum photocurrent of $12.5 \mathrm{~mA} \mathrm{~cm}^{-2}$ under AM 1.5G solar illumination, and this may be challenging to achieve when using only scalable fabrication techniques and materials.

Other earth-abundant photoanodes may perform slightly better in terms of onset potential or photocurrent ${ }^{158}$, but are not stable in highly alkaline electrolyte. The latter is desirable for the combination of separated product streams, stable earth-abundant electrocatalysts and highly conductive electrolyte (Section 2.4). Despite the conclusion of Döscher et al. ${ }^{7}$ that a tandem cell should be able to reach higher efficiencies than a triple cell (because the latter would need 1.5 times more photons to produce the same current), with the poor performance of earth-abundant photoanode/high band gap materials, the efficiency of actual tandem devices can probably easily be surpassed by silicon-based triple cells with buried junctions ${ }^{123,155}$, which approach their theoretical maximum efficiency more closely.

\subsection{Length scales}

As discussed in Chapter 2, there are several loss mechanisms in standalone solar water splitting devices, and the full integration of such a device may result in certain trade-offs. In this thesis, microstructuring was investigated for several elements of such a device, in the hope of overcoming some of these trade-offs. This is related to the length scales 
of several tens of micrometers at which some of these loss mechanisms start to play a role (or even less than a micrometer for recombination losses in earth-abundant photoelectrode materials).

In Chapter 5 it was found that micropillars are advantageous for the light absorption of a silicon (buried junction) photocathode. At larger pillar spacings these photocathodes were shown to be sensitive to catalyst loadings, but no evidence was found for limitations in charge carrier transport in the silicon or ion transport in the electrolyte, even at the largest pillar spacings of $24 \mu \mathrm{m}$. This suggests that, as long as the catalyst loading is sufficient, the catalytic centers can be placed even further apart for such photocathodes.

Charge transport in hematite, the photoanode material investigated to some extent in Chapter 6, on the other hand, is much more limited, evidenced by the fact that typically for this material layers are used that are much thinner and preferably nanostructured. Indeed the typical hole diffusion length in hematite is on the order of nanometers ${ }^{125}$, so that the loss mechanism of recombination becomes dominant at larger length scales. However the absorption of most of the solar photons with an energy above the band gap of hematite requires micrometers of optical path inside the hematite ${ }^{125}$. Therefore structures with typical dimensions of micrometers or larger that can orthogonalize the directions of light absorption and charge separation would be beneficial for such photoanodes.

In Chapter 7 mainly the limitations of ion transport were investigated, and indeed it was found to be beneficial to keep ion transport distances on the order of hundreds of micrometers or lower, to limit resistive ion transport losses. Another loss mechanism was encountered here: the perforation of solar cells seemed to increase recombination losses significantly. Because of the large charge diffusion lengths in silicon, it is easy for charge carriers to reach the defective inner walls of the pores and recombine. For hematite, which has much smaller charge carrier diffusion lengths, the charge carriers would probably recombine long before they can reach the pores, and therefore the effect of pores on hematite performance is probably negligible, except for the minor reduction in surface area. 


\subsection{Conclusion}

With the state of the art of earth-abundant semiconductor materials suitable for use in a solar-to-hydrogen device, the voltage and current needed for splitting water is probably most efficiently supplied by a silicon-based triple junction device, consisting of three stacked 'buried junction' cells. Since no semiconductor-liquid junctions are required in this case, there are few restrictions on the device configuration, and to minimize parasitic light absorption a Type D configuration would probably work best, or a version of that where the junctions and the electrolyzer are reversed so that the 'cathodic absorbers' have a higher band gap than the 'anodic absorbers'. However it is possible that great progress will be made in the performance of hematite-based photoanodes, better alkaline-stable photoelectrode materials are discovered, or progress is made with other photoanode materials in combination with advances in protection layers and/or acid-stable earthabundant catalysts. This could lead to cheaper or more efficient solar water splitting devices. In that case a configuration in which a semiconductor-liquid interface can be formed, like a Type A, B or C device, would be necessary.

Of these, a Type A device would have the least parasitic light absorption. However, as shown in Chapter 7, such devices would need micropatterned ion transport channels to avoid high ion transport losses. An advantage of these devices is that they would not need a gas separation membrane, at least not for the dimensions and current densities tested. For Type B and C devices, ion transport would be less critical, since basically the same surface area that is used for light absorption can also be used for charge transport. However these devices would then need a highly conductive alkaline-stable gas separator membrane that does not block too much light for the underlying absorber, which is still a challenge ${ }^{159}$. Still, in these devices the electrodes cannot be placed more than about a centimeter apart if the ion transport losses are to be kept small compared to the other losses. Bubble management may become an issue in such thin water layers for $\mathrm{m}^{2}$-scale devices. 


\section{Chapter 9}

\section{Societal considerations}

This chapter is based on the discussions during the workshop 'Pathways to Solar Hydrogen Technologies', held June 13-17, 2016, in the Lorentz Center in Leiden, and on the paper that was written based on these discussions ${ }^{160}$, from which the figures and tables are also reproduced.

Photoelectrochemical water splitting devices are an interesting subject for scientific study, as they require the combined understanding of optical phenomena, semiconductor physics and electrochemistry. However in this chapter it is discussed to what extent these technologies might impact society. What are possible applications, and are they economically feasible? What developments would be necessary, technologically, economically, in the public opinion, politically, and in resource use and availability, to make this technology interesting? And when can we expect these technologies to appear on the market? Of course the answers to these questions depend on many factors, but this chapter is an attempt to make some well-informed estimates.

\subsection{Scope}

The minimum requirement for a practical solar water splitting system is that it uses sunlight to convert water to a hydrogen stream that contains oxygen at a concentration below the flammability limit 20,161 . In 


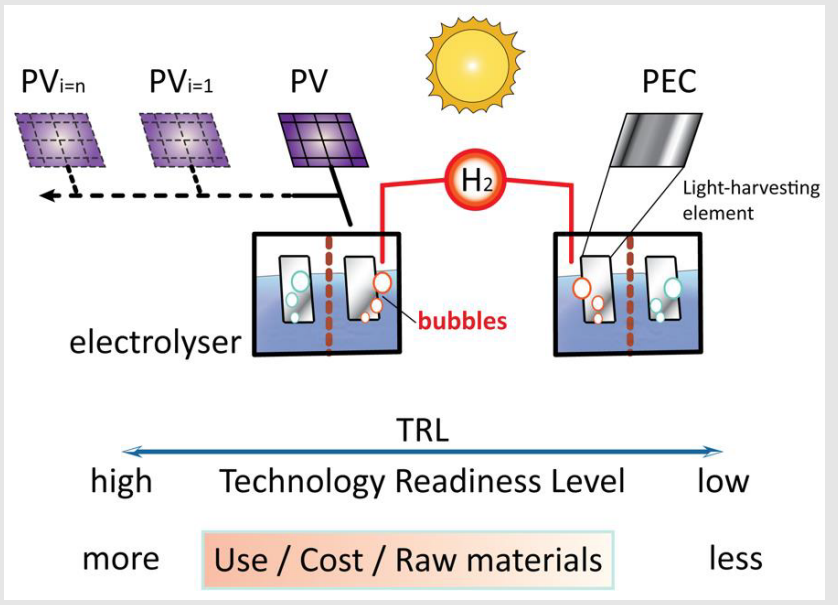

Figure 9.1: Scheme representing PEC and PV-Electrolysis device concepts, including current use, projected costs, amount of raw materials, and current relative level of technology readiness. For more details, see Table 9.1.

this Chapter, only devices and systems that generate $\mathrm{H}_{2}$ by electrochemical water splitting driven by photovoltaic power are considered. Besides fully integrated photoelectrochemical (PEC) devices, also photovoltaic panels connected to electrolyzers (PV-Electrolysis) are considered ${ }^{162-164}$. The two categories are compared in Figure 9.1 and Table 9.1. In this context, PEC devices include those based on photoelectrodes, where two half-reactions can be spatially separated by a membrane, and those based on particles suspended in an electrolyte, where the half-reactions cannot be spatially separated ${ }^{164,165}$.

For PV-Electrolysis installations it is also an option to connect them to the electricity grid, so that electrolysis can be performed using grid electricity when there is little solar irradiation (to make better use of their capital investment) ${ }^{166,167}$, or so that the PV electricity can be fed directly to the grid if the electricity demand is sufficiently high. This gridconnectedness gives the further option of connecting the two components only through the grid, and in that case they can be placed far apart (for examples the PV panels in a desert location and the electrolyzer at a hydrogen fueling station), so that long distance hydrogen 


\begin{tabular}{|c|c|c|c|}
\hline & \multicolumn{2}{|c|}{ PV-Electrolysis systems } & PEC systems \\
\hline $\begin{array}{l}\text { General con- } \\
\text { cept }\end{array}$ & \multicolumn{3}{|c|}{$\begin{array}{l}\text { Over large areas, sunlight is used to convert water to a } \\
\text { stream of hydrogen that contains an oxygen concen- } \\
\text { tration below the flammability limit }\end{array}$} \\
\hline Unique aspects & \multicolumn{2}{|c|}{$\begin{array}{l}\text { Light absorption component (PV) } \\
\text { physically separated macroscopi- } \\
\text { cally from water splitting (electrol- } \\
\text { ysis) component }\end{array}$} & $\begin{array}{l}\text { Light absorp- } \\
\text { tion and water } \\
\text { splitting com- } \\
\text { ponents are } \\
\text { integrated in } \\
\text { one region }\end{array}$ \\
\hline $\begin{array}{l}\text { Technological } \\
\text { options }\end{array}$ & $\begin{array}{l}\text { Distributed: } \\
\text { Hydrogen } \\
\text { production } \\
\text { is indepen- } \\
\text { dent of energy } \\
\text { generation (dif- } \\
\text { ferent sources, } \\
\text { electricity grid) }\end{array}$ & $\begin{array}{l}\text { Centralized: } \\
\text { Hydrogen pro- } \\
\text { duction occurs } \\
\text { at the site of } \\
\text { energy gener- } \\
\text { ation; requires } \\
\text { hydrogen } \\
\text { transport }\end{array}$ & $\begin{array}{l}\text { Centralized: } \\
\text { Design con- } \\
\text { cept exclu- } \\
\text { sively allows } \\
\text { centralized } \\
\text { operation }\end{array}$ \\
\hline $\begin{array}{l}\text { Technology } \\
\text { readiness }\end{array}$ & \multicolumn{2}{|l|}{ Advanced stage } & $\begin{array}{l}\text { Early/exploratory } \\
\text { stage }\end{array}$ \\
\hline $\begin{array}{l}\text { Economic } \\
\text { challenges }\end{array}$ & \multicolumn{3}{|c|}{$\begin{array}{l}\text { Competition with fossil and nuclear energy, battery } \\
\text { storage, and hydrogen generated by methane reform- } \\
\text { ing in terms of cost, availability, and accessibility }\end{array}$} \\
\hline $\begin{array}{l}\text { Socio-political } \\
\text { factors }\end{array}$ & \multicolumn{3}{|c|}{$\begin{array}{l}\text { Investments depend on societal acceptance and lo- } \\
\text { cal and international politics, which are in turn influ- } \\
\text { enced by accidents (in hydrogen and competing in- } \\
\text { dustries), interest groups, fossil fuel availability, climate } \\
\text { change }\end{array}$} \\
\hline
\end{tabular}

Table 9.1: PV-Electrolysis versus PEC systems. Overview of general concepts, and comparison of unique characteristics, technological considerations, economic challenges and political factors for each device type. 
transport is not necessary. The latter is here termed a distributed approach, as opposed to the centralized approach where the light absorption and electrolysis happen at the same location (be it by PEC or PV-Electrolysis).

\subsection{Technical options and merits}

So far in this thesis, mostly PEC devices with advanced microstructures have been discussed. However they are somewhere in the middle of a spectrum that ranges from at most macroscopically integrated systems, based on technology that is currently on the market, with little potential for cost reduction on the one side, to highly experimental systems with advanced structuring/integration below the micrometer scale, potentially disruptive for the cost of hydrogen on the other side of the spectrum ${ }^{162}$. Since one end of this spectrum will likely precede PEC devices when it comes to prototypes and scale-up, and the other end has more potential for making cheap solar hydrogen, the specifics and merits of the various parts of the spectrum are discussed here.

The PV-Electrolysis part of the spectrum consists of modular systems: the PV panel and the electrolyzer are separate modules. This has the advantage that each module can be optimized separately, with hardly any compatibility considerations ${ }^{168,169}$. Power converters (DCDC or DC-AC and AC-DC) can be used to have each module work at the most economic point in its current-voltage characteristic, especially in the grid-connected case when surpluses or shortages can be leveled with grid power 166,170. All of these technologies are currently commercially available, and especially PV panels benefit from the price benefits of economies of scale ${ }^{171-173}$. Therefore short-term ( $<10$ years) solar hydrogen applications will probably use this technology. However, for PV-Electrolysis the different modules always have to be packaged separately, and the power converters are costly and have an efficiency below unity, indicating that further integration has the potential of further price reduction. Even considering the potential of economies of scale, solar hydrogen based on current technology would at least be three times as expensive as energy from fossil fuels 167.

The solar panel modules for PV-Electrolysis will probably be the currently ubiquitous silicon solar cells, although the developments in CdTe 
and CIGS photovoltaics are likely to result in similar prices ${ }^{100}$. Price reductions in III-V semiconductor materials or developments in hybrid organic-inorganic halide perovskites or tandem architectures could be disruptive to the PV space ${ }^{174,175}$. For water electrolysis there are currently two options: alkaline electrolyzers and proton-exchange membrane (PEM) electrolyzers. The currently emerging solid-oxide fuel cells might be viable options in the longer term. Liquid electrolyte alkaline electrolyzers have been deployed commercially for more than hundred years ${ }^{21,176}$. Because of this, they have already been developed and implemented on larger scales than PEM electrolyzers. However they are typically less efficient than PEM electrolyzers, and have more safety concerns because they typically use porous gas separation membranes and a corrosive liquid electrolyte. Since the crossover is caused by diffusion of dissolved gases and the concentration gradient is limited by the solubility of the gases, crossover is more or less constant for all operation currents, so that the ratio of crossover to production changes with operation current, and could become dangerously high for low operation currents that will occur when there is less or no sunshine. This might be solved by using batteries or grid electricity to keep the electrolysis at a minimum level, by flushing/degassing the electrolytes when the current becomes too low, or other engineering approaches $161,177,178$. The development of cation exchange membranes for alkaline electrolyzers could reduce the need for such measures ${ }^{159}$. PEM electrolyzers have an advantage here since they already use highly conductive proton exchange membranes as electrolyte and gas separator. However the state of the art catalysts for PEM electrolyzers are platinum and iridium oxide, which would prohibit implementation at the energy market scale. Also they are currently more costly, but this may change as their production volumes increase, or lower catalyst loadings or earth-abundant alternatives are developed. Moreover, since the capital cost of an electrolyzer is small compared to the cost of the photovoltaics 168 , an efficiency increase of the electrolyzer could greatly reduce the hydrogen price because fewer solar panels are needed to produce the same amount of hydrogen. For initial, small-scale projects the PEM electrolyzers are probably anyway the most suitable.

A bit more integrated, but still PV-Electrolysis, is the case where the solar panels are directly connected to the electrolyzers. Both components have to be matched to each other in terms of their current- 
voltage characteristic, and have to be placed at the same location (or a new DC power infrastructure has to be built). An efficiency of $14 \%$ has already been shown on a laboratory scale for this concept ${ }^{179}$.

PEC devices are the next category in the spectrum. Closest to the directly connected form of PV-Electrolysis are the PEC devices that are basically modified PV panels: semiconductor panels where the water splitting reaction takes place directly on the surface of the semiconductor or on a layer integrated on this surface. These devices can have a semiconductor-liquid junction or a buried junction. This means that all panels need to support an electrolyte layer over at least part of their surface, and need electrolyte and gas management systems. The gas management is quite different from PV-Electrolysis, since the amount of electrochemically active surface would be $\sim 50$ times larger than its equivalent PV-Electrolysis system ${ }^{162,168}$, and therefore the same amount of gas is produced over a much larger area. This could have the advantage of higher operating efficiencies and less stringent catalyst requirements ${ }^{180}$. Because of the planar approach these devices may still benefit from fabrication technology (and prices) from photovoltaics industry ${ }^{181}$. Their main potential for price reduction lies in the reduced amount of packaging and electricity transformation/transport. However, given the advantages of the modularity of PV-Electrolysis, this type of PEC devices would probably only be preferred if the used semiconductors work better as photoelectrodes than as PV materials. Also, they may have issues with parasitic light absorption by the catalysts, which is addressed in an innovative way in Chapter 5, and with product gas bubbles interfering with light absorption and catalysis ${ }^{182}$. Planar PEC devices are not expected to be ready for the market within 10 years, as in current demonstrations PV-Electrolysis outperforms these devices in terms of both efficiency and stability ${ }^{180}$.

In this thesis it has been shown that advanced structuring, for example to make devices with vertically patterned micropillars and microporous membranes, can circumvent some of the trade-offs that may

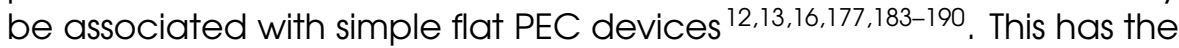
potential of drastic efficiency improvements, but it remains to be seen if this outweighs the extra costs associated with more advanced fabrication techniques. This technology is not even ready to come out of the lab, so these microstructured PEC devices will probably take even longer to develop than the flat PEC systems.

Even more experimental are the particulate and molecular systems, 
where suspensions of PEC nanoparticles or solutions of photoactive molecules produce the hydrogen and oxygen upon solar illumination. However if these particles can be mass produced cheaply, they can lead to cheaper systems that do not require the support of rigid panels/packaging 94,191-193. On the downside, more than the other systems this type of technology faces serious challenges with charge transport and gas separation ${ }^{192,194-196 .}$

\subsection{Economic considerations}

The product of solar water splitting is hydrogen, and for economical viability this hydrogen has to be at least comparable in price with the current standard, hydrogen produced by methane steam reforming ${ }^{197}$, which is used for petroleum refining and ammonia and methanol production. Since methane is a fossil fuel and the conversion process produces $\mathrm{CO}_{2}$, this method has additional costs (climate change) that are not currently included in the hydrogen price. These costs could be taken into account by applying proper taxes ${ }^{198}$. This would make it easier for solar hydrogen to become price competitive.

Should hydrogen however not only be seen as a chemical feedstock, but also as a source of energy or a fuel, then it has to compete with energy produced from other sources, for example fossil, nuclear, hydroelectric and wind. Given the scale of the energy market this is an enormous opportunity, but it also puts even more stringent requirements on the price of the produced hydrogen and the scalability of the fabrication methods and materials used ${ }^{199-201}$. For short-term energy storage, as a means to deal with the intermittent nature of solar energy, it has to compete with battery storage, which will be hard because of the energetic losses of around $30 \%$ in both the electrolysis (hydrogen generation) step and the recombination (electricity generation) step. It can be considered for storing the surplus solar energy of the summer for the winter with its higher heating needs, since selfdischarge limits the lifetime of battery-stored electricity. Also for long distance transportation it may be more useful than battery storage because of its higher gravimetric energy density.

In the long term, with policies that encourage the use of renewable energy, and fossil fuel reserves depleting, solar hydrogen may 
become cost-competitive with fossil fuels. But already before solar hydrogen can compete on these markets, it may have advantages over traditional sources of hydrogen and energy for certain niche markets, where for example the purity (hydrogen from methane reforming contains carbon species impurities), zero emission, local generation or grid-independence are more important than the price. It can for example be a feedstock for the production of high margin $\mathrm{CO}_{2}$ neutral products, or serve as the energy harvesting and storage system for small, grid independent centers like islands, military bases or space stations. Besides that, advances in the component technologies themselves could impact other industries involved in the electrochemical production of alternative commodity chemicals to hydrogen (e.g. chloralkali, zinc production, aluminum production) ${ }^{202}$ or on electrochemical wastewater treatment ${ }^{203}$. These industries enjoy higher margins than the energy industry and already use electrochemical methods for large scale production, ${ }^{204}$, which could facilitate early stage implementation of solar hydrogen technologies.

\subsection{Societal and political impacts}

Public opinion is crucial for the successful implementation of any technology, also for solar hydrogen. Not only because it directly influences the demand for solar hydrogen, but also because it influences political decisions. Government investment in research, infrastructure and subsidies can make all the difference in getting this technology through its early stages, as do specific taxes, subsidies, regulations and agreements, such as the Paris Climate Agreement ${ }^{205}$ and the Dutch target of $40 \%$ renewable energy by 2030 and a $>80 \%$ reduction in $\mathrm{CO}_{2}$ emissions by $2050^{206}$.

For the public, price and safety are the most important concerns 207 . Accidents with hydrogen or competing technologies can quickly change the public opinion in favor or against hydrogen technology 208,209, and as the effects of climate change and pollution become more and more visible, more and more people are expected to favor renewable energy 210-212. However then solar hydrogen still has to compete with other renewable energy technologies and technologies that promise to reduce energy consumption or net $\mathrm{CO}_{2}$ emissions. 


\subsection{Conclusions and perspectives}

This Chapter has presented a perspective on expected and necessary developments in solar hydrogen technologies, considering a broader spectrum of technologies that could achieve this. Within 10 years, the only applications of solar hydrogen will probably be certain niche markets. Silicon solar panels connected to proton exchange membrane electrolyzers will be the technology of choice for these applications and this time frame, because they are state-of-the-art technologies that are already on the market. Developments that could make this technology more attractive mainly concern electrolyzers that can deal better with the intermittent power input inherent to solar electricity. Efficiency increases for the electrolyzers could also help, as well as matching of the solar panels with the electrolyzers so that power electronics can be avoided.

In the medium term, solar hydrogen could replace hydrogen produced by methane steam reforming as a chemical feedstock or perhaps as a clean energy source for long distance transportation. Disruptive developments in solar hydrogen as well as policies and infrastructure that encourage the use of renewable energy are necessary to make this an economically viable option. Disruptive developments would be new photovoltaic technology that can produce electricity more cheaply or efficiently than current silicon solar panels, or stable, efficient and cheaply produced photoelectrochemical water splitting devices with materials that are not useful for photovoltaics. Policies and infrastructure, as well as consumer demand for hydrogen-powered vehicles will largely depend on public opinion, which will depend on many factors that are hard to predict.

For the longer term and larger scale, solar hydrogen has to compete on the energy market with fossil fuels, nuclear power, and other renewable energy sources. Depletion of fossil fuels and increasing awareness of the adverse effects of climate change could work in favor of renewable sources in general. Still, the price and scale requirements of this market are greater than that of hydrogen as a chemical feedstock. As for the case of solar panels connected to electrolyzers, there is simply not enough iridium and platinum on this earth to use proton exchange membrane electrolyzers on this scale. Earth-abundant acidstable catalysts have to be found, or better anion exchange membranes for alkaline electrolyzers have to be developed. Integrated, 
photoelectrochemical devices with advanced structuring, or particlebased systems have the potential of providing the cost reductions that are needed. However they are still in a very early stage of development, and many issues related to efficiency, gas separation, selective catalysis, stability, and fabrication costs have to be resolved should these devices ever provide a viable alternative. Therefore it is uncertain whether solar hydrogen will ever be deployed on this scale. 


\section{Bibliography}

(1) C. F. Keller. Global warming: a review of this mostly settled issue. Stochastic Environmental Research and Risk Assessment, 23(5): 643-676, Jul 2009. ISSN 1436-3259. URL https ://doi .org/10.1007/ s00477-008-0253-3.

(2) G.-R. Walther. Community and ecosystem responses to recent climate change. Philosophical Transactions of the Royal Society B: Biological Sciences, 365(1549):2019-2024, 2010. URL http:// dx.doi.org/10.1098/rstb. 2010.0021.

(3) V. Scott, R. S. Haszeldine, S. F. B. Tett, and A. Oschlies. Fossil fuels in a trillion tonne world. Nature Climate Change, 5(5):419-423, 2015. URL http://dx.doi.org/10.1038/nclimate2578.

(4) O. Ellabban, H. Abu-Rub, and F. Blaabjerg. Renewable energy resources: Current status, future prospects and their enabling technology. Renewable and Sustainable Energy Reviews, 39 (Supplement C):748 - 764, 2014. ISSN 1364-0321. URL https: //doi.org/10.1016/j.rser.2014.07.113.

(5) M. Göłz, J. Lefebvre, F. Mörs, A. M. Koch, F. Graf, S. Bajohr, R. Reimert, and T. Kolb. Renewable power-to-gas: A technological and economic review. Renewable Energy, 85(Supplement C): 1371 - 1390, 2016. ISSN 0960-1481. URL https://doi.org/10. 1016/j.renene. 2015.07.066.

(6) R. Elbersen, R. M. Tiggelaar, A. Milbrat, G. Mul, H. Gardeniers, and J. Huskens. Controlled doping methods for radial $p / n$ junctions in silicon. Advanced Energy Materials, 5(6):1401745, 2015. ISSN 1614-6840. URL http://dx.doi.org/10.1002/aenm. 201401745. 
(7) H. Döscher, J. Geisz, T. Deutsch, and J. Turner. Sunlight absorption in water-efficiency and design implications for photoelectrochemical devices. Energy and Environmental Science, 7(9): 2951-2956, 2014. URL http://dx.doi. org/10.1039/c4ee01753f.

(8) A. Polman, M. Knight, E. C. Garnett, B. Ehrler, and W. C. Sinke. Photovoltaic materials: Present efficiencies and future challenges. Science, 352(6283), 2016. ISSN 0036-8075. URL http: //dx.doi.org/10.1126/science. aad4424.

(9) K. T. Fountaine, H. J. Lewerenz, and H. A. Atwater. Efficiency limits for photoelectrochemical water-splitting. Nature Communications, 7, 2016. URL https://doi .org/10.1038/ncomms 13706.

(10) I. Yahyaie, K. McEleney, M. G. Walter, D. R. Oliver, D. J. Thomson, M. S. Freund, and N. S. Lewis. Characterization of the electrical properties of individual p-si microwire/polymer/n-si microwire assemblies. The Journal of Physical Chemistry C, 115(50):2494524950, 2011. URL http://dx.doi.org/10.1021/jp2088719.

(11) P. Ghosh, S. Satou, H. Nakamori, T. Noda, I. Daisuke, and M. Tanemura. Facile one-step fabrication of highly transparent and flexible superhydrophobic substrate by room-temperature ion irradiation method. physica status solidi (RRL) - Rapid Research Letters, 6(11):430-432, 2012. ISSN 1862-6270. URL http://dx.doi.org/10. 1002/pssr. 201206418.

(12) M. R. Singh, K. Papadantonakis, C. Xiang, and N. S. Lewis. An electrochemical engineering assessment of the operational conditions and constraints for solar-driven water-splitting systems at near-neutral pH. Energy and Environmental Science, 8(9):27602767, 2015. URL http://dx.doi.org/10.1039/c5ee01721a.

(13) M. R. Singh, C. Xiang, and N. S. Lewis. Evaluation of flow schemes for near-neutral $\mathrm{pH}$ electrolytes in solar-fuel generators. Sustainable Energy Fuels, 1:458-466, 2017. URL http://dx.doi.org/10. 1039/C7SE00062F.

(14) Y. Chen, S. Hu, C. Xiang, and N. S. Lewis. A sensitivity analysis to assess the relative importance of improvements in electrocatalysts, light absorbers, and system geometry on the efficiency of solar-fuels generators. Energy Environ. Sci., 8:876-886, 2015. URL http://dx.doi.org/10.1039/C4EE02314E. 
(15) S. Y. Reece, J. A. Hamel, K. Sung, T. D. Jarvi, A. J. Esswein, J. J. H. Pijpers, and D. G. Nocera. Wireless solar water splitting using silicon-based semiconductors and earth-abundant catalysts. Science, 334(6056):645-648, 2011. ISSN 0036-8075. URL http://science.sciencemag.org/content/334/6056/645.

(16) K. Walczak, Y. Chen, C. Karp, J. W. Beeman, M. Shaner, J. Spurgeon, I. D. Sharp, X. Amashukeli, W. West, J. Jin, N. S. Lewis, and $C$. Xiang. Modeling, simulation, and fabrication of a fully integrated, acid-stable, scalable solar-driven water-splitting system. ChemSusChem, 8(3):544-551, 2015. ISSN 1864-564X. URL http://dx.doi.org/10.1002/cssc. 201402896.

(17) T. Bosserez, L. Geerts, J. Rong??, F. Ceyssens, S. Haussener, R. Puers, and J. A. Martens. Minimization of ionic transport resistance in porous monoliths for application in integrated solar water splitting devices. The Journal of Physical Chemistry C, 120(38):21242-21247, 2016. URL http://dx.doi.org/10.1021/acs . jpcc. $6 \mathrm{~b} 06766$.

(18) C. Trompoukis, A. Abass, J.-W. Schüttauf, T. Bosserez, J. Rongé, J. Lauwaert, J. A. Martens, and R. Baets. Porous multi-junction thin-film silicon solar cells for scalable solar water splitting. Solar Energy Materials and Solar Cells, 182:196 - 203, 2018. ISSN 09270248. URL https://doi.org/10.1016/j.solmat.2018.03.041.

(19) A. Hankin, F. E. Bedoya-Lora, C. K. Ong, J. C. Alexander, F. Petter, and G. H. Kelsall. From millimetres to metres: the critical role of current density distributions in photo-electrochemical reactor design. Energy Environ. Sci., 10:346-360, 2017. URL http: //dx.doi.org/10.1039/C6EE03036J.

(20) V. Schröder, B. Emonts, H. Janßen, and H.-P. Schulze. Explosion limits of hydrogen/oxygen mixtures at initial pressures up to 200 bar. Chemical Engineering \& Technology, 27(8):847-851, 2004. ISSN 1521-4125. URL http://dx.doi.org/10.1002/ceat. 200403174.

(21) D. M. F. Santos, C. A. C. Sequeira, and J. L. Figueiredo. Hydrogen production by alkaline water electrolysis. Química Nova, 36:1176 - 1193, 00 2013. ISSN 0100-4042. URL http://dx.doi.org/10.1590/ S0100-40422013000800017. 
(22) J. T. Davis, J. Qi, X. Fan, J. C. Bui, and D. V. Esposito. Floating membraneless pv-electrolyzer based on buoyancy-driven product separation. International Journal of Hydrogen Energy, pages -, 2017. ISSN 0360-3199. URL https://doi.org/10.1016/j. ijhydene.2017.11.086.

(23) S. M. H. Hashemi, M. A. Modestino, and D. Psaltis. A membraneless electrolyzer for hydrogen production across the ph scale. Energy Environ. Sci., 8:2003-2009, 2015. URL http://dx.doi.org/10. 1039/C5EE00083A.

(24) P. J. Westerik, W. J. C. Vijselaar, J. W. Berenschot, N. R. Tas, J. Huskens, and J. G. E. Gardeniers. Sidewall patterning-a new wafer-scale method for accurate patterning of vertical silicon structures. Journal of Micromechanics and Microengineering, 28(1):015008, 2017. URL https://doi.org/10.1088/1361-6439/ aa9c20.

(25) J. Jeon, H. C. Floresca, and M. J. Kim. Fabrication of complex three-dimensional nanostructures using focused ion beam and nanomanipulation. Journal of Vacuum Science \& Technology $B$, Nanotechnology and Microelectronics: Materials, Processing, Measurement, and Phenomena, 28(3):549-553, 2010. URL http: $/ / \mathrm{dx}$. doi.org/10.1116/1.3406134.

(26) Y. Ma, A. Pan, J. Si, T. Chen, F. Chen, and X. Hou. A simple method for fabrication of high-aspect-ratio all-silicon grooves. Applied Surface Science, 284:372 - 378, 2013. ISSN 0169-4332. URL http: //dx.doi.org/10.1016/j.apsusc. 2013.07.107.

(27) M. Vaezi, H. Seitz, and S. Yang. A review on 3d micro-additive manufacturing technologies. The International Journal of Advanced Manufacturing Technology, 67(5):1721-1754, 2013. ISSN 1433-3015. URL http://dx.doi.org/10.1007/s00170-012-4605-2.

(28) S. Azimi, Z. Y. Dang, K. Ansari, and M. B. H. Breese. Fabrication of silicon molds with multi-level, non-planar, micro- and nano-scale features. Nanotechnology, 25(37), 2014. URL http://dx.doi.org/ $10.1088 / 0957-4484 / 25 / 37 / 375301$.

(29) C. Schelling. Manufacturing method for a micromechanical component and a corresponding micromechanical compo- 
nent, June 12 2014. URL http://www.google.com.gt/patents/ US20140159209. US Patent App. 14/098,879.

(30) J. R. Raney and J. A. Lewis. Printing mesoscale architectures. MRS Bulletin, 40(11):943-950, 2015. URL http://dx.doi.org/10.1557/ mrs. 2015.235. Cited By :8.

(31) H. Li. Microfabrication techniques for producing freestanding multi-dimensional microstructures. Microsystem Technologies, 22(2):223-237, 2016. URL http://dx.doi.org/10.1007/ s00542-015-2720-3.

(32) C. M. Waits, B. Morgan, M. Kastantin, and R. Ghodssi. Microfabrication of $3 d$ silicon mems structures using gray-scale lithography and deep reactive ion etching. Sensors and Actuators, A: Physical, 119(1):245-253, 2005. URL http://dx.doi.org/10.1016/ j.sna.2004.03.024.

(33) G. Calafiore, A. Koshelev, F. I. Allen, S. Dhuey, S. Sassolini, E. Wong, P. Lum, K. Munechika, and S. Cabrini. Nanoimprint of a $3 d$ structure on an optical fiber for light wavefront manipulation. Nanotechnology, 27(37), 2016. URL http://dx.doi.org/10.1088/ $0957-4484 / 27 / 37 / 375301$.

(34) J. Jang, C. K. Ullal, M. Maldovan, T. Gorishnyy, S. Kooi, C. Y. Koh, and E. L. Thomas. 3d micro- and nanostructures via interference lithography. Advanced Functional Materials, 17(16):3027-3041, 2007. URL http://dx.doi.org/10.1002/adfm.200700140.

(35) N. Roxhed, P. Griss, and G. Stemme. Reliable in-vivo penetration and transdermal injection using ultra-sharp hollow microneedles. In The 13th International Conference on Solid-State Sensors, Actuators and Microsystems, 2005. Digest of Technical Papers. TRANSDUCERS '05., volume 1, pages 213-216 Vol. 1, June 2005. URL http://dx.doi.org/10.1109/SENSOR. 2005.1496396.

(36) C.-H. Choi and C.-J. Kim. Fabrication of a dense array of tall nanostructures over a large sample area with sidewall profile and tip sharpness control. Nanotechnology, 17(21):5326, 2006. URL http://dx.doi.org/10.1088/0957-4484/17/21/007. 
(37) K. Hirose, F. Shiraishi, and Y. Mita. A simultaneous vertical and horizontal self-patterning method for deep three-dimensional microstructures. Journal of Micromechanics and Microengineering, 17(7):S68, 2007. URL http://dx.doi.org/10.1088/0960-1317/17/ $7 / \mathrm{S} 02$.

(38) G. Sun, T. Gao, X. Zhao, and H. Zhang. Fabrication of micro/nano dual-scale structures by improved deep reactive ion etching. Journal of Micromechanics and Microengineering, 20(7):075028, 2010. URL http://dx.doi.org/10.1088/0960-1317/20/7/075028.

(39) S. S. Walavalkar, A. P. Homyk, M. D. Henry, and A. Scherer. Three-dimensional etching of silicon for the fabrication of lowdimensional and suspended devices. Nanoscale, 5:927-931, 2013. URL http://dx.doi.org/10.1039/C2NR32981F.

(40) Y. M. Sabry, D. Khalil, B. Saadany, and T. Bourouina. Multi-step etching of three-dimensional sub-millimeter curved silicon microstructures with in-plane principal axis. Microelectronic Engineering, 114:78 - 84, 2014. ISSN 0167-9317. URL http://dx.doi. org $/ 10.1016 / j$.mee. 2013.10.001.

(41) M. S. Gudiksen, L. J. Lauhon, J. Wang, D. C. Smith, and C. M. Lieber. Growth of nanowire superlattice structures for nanoscale photonics and electronics. Nature, 415(6872):617-620, 2002. URL http://dx.doi.org/10.1038/415617a.

(42) W. Lu, P. Xie, and C. M. Lieber. Nanowire transistor performance limits and applications. IEEE Transactions on Electron Devices, 55 (11):2859-2876, Nov 2008. ISSN 0018-9383. URL http://dx.doi. org/10.1109/TED.2008.2005158.

(43) R. Yan, D. Gargas, and P. Yang. Nanowire photonics. Nature Photonics, 3(10):569-576, 2009. URL http://dx.doi.org/10.1038/ nphot on.2009.184.

(44) K. Sun, A. Kargar, N. Park, K. N. Madsen, P. W. Naughton, T. Bright, Y. Jing, and D. Wang. Compound semiconductor nanowire solar cells. IEEE Journal of Selected Topics in Quantum Electronics, 17 (4): 1033-1049, July 201 1. ISSN 1077-260X. URL http://dx.doi.org/ 10.1109/JSTQE. 2010.2090342. 
(45) J. K. Hyun, S. Zhang, and L. J. Lauhon. Nanowire heterostructures. Annual Review of Materials Research, 43(1):451-479, 2013. URL http://dx.doi.org/10.1146/annurev-matsci-071312-121659.

(46) Y. Wang, T. Wang, P. Da, M. Xu, H. Wu, and G. Zheng. Silicon nanowires for biosensing, energy storage, and conversion. Advanced Materials, 25(37):5177-5195, 2013. ISSN 1521-4095. URL http://dx.doi.org/10.1002/adma.201301943.

(47) L. Wen, Z. Wang, Y. Mi, R. Xu, S.-H. Yu, and Y. Lei. Designing heterogeneous $1 d$ nanostructure arrays based on aao templates for energy applications. Small, 11(28):3408-3428, 2015. ISSN 16136829. URL http://dx.doi.org/10.1002/smll.201500120.

(48) C. W. Pinion, J. D. Christesen, and J. F. Cahoon. Understanding the vapor-liquid-solid mechanism of si nanowire growth and doping to synthetically encode precise nanoscale morphology. J. Mater. Chem. C, 4:3890-3897, 2016. URL http://dx.doi.org/10. 1039/C5TC03898G.

(49) Q. Li, S. Ding, W. Zhu, L. Feng, H. Dong, and W. Hu. Recent advances in one-dimensional organic $p-n$ heterojunctions for optoelectronic device applications. J. Mater. Chem. C, 4:9388-9398, 2016. URL http://dx.doi.org/10.1039/C6TC03280J.

(50) T. Ozel, G. R. Bourret, and C. A. Mirkin. Coaxial lithography. Nature Nanotechnology, 10(4):319-324, 2015. URL http://dx.doi.org/ 10.1038/nnano. 2015.33.

(51) T. G. Leong, A. M. Zarafshar, and D. H. Gracias. Three-dimensional fabrication at small size scales. Small, 6(7):792-806, 2010. ISSN 1613-6829. URL http://dx.doi.org/10.1002/smll. 200901704.

(52) M. De Volder, S. H. Tawfick, S. J. Park, D. Copic, Z. Zhao, W. Lu, and A. J. Hart. Diverse $3 \mathrm{~d}$ microarchitectures made by capillary forming of carbon nanotubes. Advanced Materials, 22(39): 4384-4389, 2010. ISSN 1521-4095. URL http://dx.doi.org/10. 1002/adma. 201001893

(53) B. Dinesh, A. Bianco, and C. Menard-Moyon. Designing multimodal carbon nanotubes by covalent multi-functionalization. Nanoscale, 8:18596-18611, 2016. URL http://dx.doi.org/10. 1039/C6NR06728J. 
(54) K. E. Petersen. Silicon as a mechanical material. Proceedings of the IEEE, 70(5):420-457, 1982. URL http://dx.doi.org/10.1109/ PROC. 1982.12331.

(55) Y. Yoon. Doping method using an oxide film and a nitride film on the trench wall to manufacture a semiconductor device and the manufactured device, March 12 1991. URL https://www.google. nl/patents/US4999312. US Patent 4,999,312.

(56) K. A. Shaw, Z. L. Zhang, and N. C. MacDonald. Scream i: A single mask, single-crystal silicon, reactive ion etching process for microelectromechanical structures. Sensors and Actuators: A.Physical, 40(1):63-70, 1994. URL http://dx.doi.org/10.1016/ 0924-4247 (94) 85031-3.

(57) B. D. Gates, Q. Xu, M. Stewart, D. Ryan, C. G. Willson, and G. M. Whitesides. New approaches to nanofabrication: Molding, printing, and other techniques. Chemical Reviews, 105(4):1171-1196, 2005. URL http://dx.doi.org/10.1021/cr030076o.

(58) E. Sarajlic, E. Berenschot, G. Krijnen, and M. Elwenspoek. Fabrication of $3 d$ nanowire frames by conventional micromachining technology. In 13th International Conference on Solid-State Sensors, Actuators and Microsystems, TRANSDUCERS 2005, volume 1, pages 27-30. IEEE, 2005. URL https://doi.org/10.1109/SENSOR. 2005.1496350.

(59) P. Abgrall and A.-M. Gué. Lab-on-chip technologies: making a microfluidic network and coupling it into a complete microsystem-a review. Journal of Micromechanics and Microengineering, 17(5):R15, 2007. URL http://dx.doi.org/10.1088/ 0960-1317/17/5/R01.

(60) E. Berenschot, N. R. Tas, H. V. Jansen, and M. Elwenspoek. 3dnanomachining using corner lithography. In 2008 3rd IEEE International Conference on Nano/Micro Engineered and Molecular Systems, pages 729-732, Jan 2008. URL http://dx.doi.org/10. 1109/NEMS. 2008.4484432.

(61) J. W. Berenschot, N. R. Tas, H. V. Jansen, and M. Elwenspoek. Chemically anisotropic single-crystalline silicon nanotetrahedra. Nanotechnology, 20(47):475302, 2009. URL http://dx.doi.org/ $10.1088 / 0957-4484 / 20 / 47 / 475302$. 
(62) J. von Kluge, A. Scholz, J. Radecker, M. Patz, S. Kudelka, and A. Avellan. Insulator material over buried conductive line, December 31 2009. URL https://www.google.nl/patents/ US20090321805. US Patent App. 12/165,072.

(63) C. Shen, H. T. M. Pham, and P. M. Sarro. A multifunctional vertical microsieve for micro and nano particles separation. In Micro Electro Mechanical Systems, 2009. MEMS 2009. IEEE 22nd International Conference on, pages 383-386, Jan 2009. URL http://dx.doi.org/10.1109/MEMSYS. 2009.4805399.

(64) A. Cohen, R. Chen, U. Frodis, M. Wu, and C. Folk. Waferscale, solid freeform fabrication of fully-assembled metal micromechanisms for minimally-invasive medical devices. In 20th Annual International Solid Freeform Fabrication Symposium, SFF 2009, pages 620-630, 2009. URL http://sffsymposium.engr. utexas . edu/Manuscripts/2009/2009-53-Cohen.pdf.

(65) Y. Zhang, A. Toda, J. Lu, H. Okada, T. Kobayashi, T. Itoh, and R. Maeda. Wafer-scale mems technology of new vertically laminated cantilevers. Procedia Engineering, 25:677 - 680, 2011. ISSN 1877-7058. URL http://dx.doi.org/10.1016/j.proeng. 2011. 12.167.

(66) E. J. W. Berenschot, N. Burouni, B. Schurink, J. W. Van Honschoten, R. G. P. Sanders, R. Truckenmuller, H. V. Jansen, M. C. Elwenspoek, A. A. Van Apeldoorn, and N. R. Tas. 3d nanofabrication of fluidic components by corner lithography. Small, 8(24):3823-3831, 2012. URL http://dx.doi.org/10.1002/smll.201201446.

(67) E. J. W. Berenschot, H. V. Jansen, and N. R. Tas. Fabrication of $3 \mathrm{~d}$ fractal structures using nanoscale anisotropic etching of single crystalline silicon. Journal of Micromechanics and Microengineering, 23(5):055024, 2013. URL http://dx.doi.org/10.1088/ 0960-1317/23/5/055024.

(68) A. Sandoughsaz, S. Azimi, H. Mazreati, and S. Mohajerzadeh. Realization of complex three-dimensional free-standing structures on silicon substrates using controllable underetching in a deep reactive ion etching. Journal of Micromechanics and Microengineering, 23(3):035022, 2013. URL http://dx.doi.org/10.1088/ $0960-1317 / 23 / 3 / 035022$. 
(69) E. J. W. Berenschot, H. Yagubizade, H. V. Jansen, M. Dijkstra, and N. R. Tas. Fabrication of $2 d$-extruded fractal structures using repeated corner lithography and etching. In The 9th IEEE International Conference on Nano/Micro Engineered and Molecular Systems (NEMS), pages 374-377, April 2014. URL http://dx.doi. org/10.1109/NEMS. 2014.6908830.

(70) J. W. Berenschot, R. M. Tiggelaar, J. Geerlings, J. G. E. Gardeniers, N. R. Tas, M. Malankowska, M. P. Pina, and R. Mallada. $3 d$-fractal engineering based on oxide-only corner lithography. In 2016 Symposium on Design, Test, Integration and Packaging of MEMS/MOEMS (DTIP), pages 1-4, May 2016. URL http: //dx.doi.org/10.1109/DTIP.2016.7514895.

(71) A. Milbrat, R. Elbersen, R. Kas, R. M. Tiggelaar, H. Gardeniers, G. Mul, and J. Huskens. Spatioselective electrochemical and photoelectrochemical functionalization of silicon microwires with axial p/n junctions. Advanced Materials, 28(7): 1400-1405, 2016. ISSN 1521-4095. URL http://dx.doi.org/10.1002/adma.201504609.

(72) J. Berenschot, X. Sun, H. L. The, R. T. abd M.J. de Boer abd J.C.T. Eijkel abd J.G.E Gardeniers, and N. Tas. Wafer-scale nanostructure formation inside vertical nano-pores. In The 12th IEEE International Conference on Nano/Micro Engineered and Molecular Systems (NEMS), pages 57-60, April 2017.

(73) Y. Yoon and C. Bok. Method for manufacturing a trench capacitor using a photoresist etch back process, February 19 1991. URL https://www. google.nl/patents/US4994409. US Patent 4,994,409.

(74) K. Golden, P. Pan, K. Stewart, and A. Thomas. Method of making single-step trenches using resist fill and recess, April 8 1997. URL https://www .google.nl/patents/US5618751. US Patent 5,618,751.

(75) S. Kudelka, A. Michaelis, and D. Tobben. Method for expanding trenches by an anisotropic wet etch, July 30 2002. URL https: //www . google.nl/patents/US6426254. US Patent 6,426,254.

(76) H. Tews, S. Kudelka, and I. McStay. Process flow for sacrificial collar with poly mask, October 1 2002. URL https://www.google.nl/ patents/US6458647. US Patent 6,458,647. 
(77) S. Lin, Y. Hsu, and C. Lee. Method of etching bottle trench and fabricating capacitor with same, September 22 2005. URL https://www.google.nl/patents/US20050208727. US Patent App. 10/871,619.

(78) C. Sun, T. Huang, and C. Wu. Method for forming bottle-shaped trench, September 5 2006. URL https://www.google.nl/patents/ US7101802. US Patent 7,101,802.

(79) L. F. Voss, Q. Shao, C. E. Reinhardt, R. T. Graff, A. M. Conway, R. J. Nikolić, N. Deo, and C. L. Cheung. Planarization of high aspect ratio $\mathrm{p}$-i-n diode pillar arrays for blanket electrical contacts. Journal of Vacuum Science \& Technology B, 28(5):916-920, 2010. URL http://dx.doi.org/10.1116/1.3478306.

(80) E. Warren, J. McKone, H. Atwater, H. Gray, and N. Lewis. Hydrogen-evolution characteristics of ni-mo-coated, radial junction, $\mathrm{n}+\mathrm{p}$-silicon microwire array photocathodes. Energy and Environmental Science, 5(11):9653-9661, 2012. URL http://dx.doi. org/10.1039/c2ee23192a.

(81) C. Liu, J. Tang, H. M. Chen, B. Liu, and P. Yang. A fully integrated nanosystem of semiconductor nanowires for direct solar water splitting. Nano Letters, 13(6):2989-2992, 2013. URL http://dx.doi. org/10.1021/n1401615t.

(82) J. Liu, Y. C. Tai, J. Lee, K. C. Pong, Y. Zohar, and C. M. Ho. In situ monitoring and universal modelling of sacrificial psg etching using hydrofluoric acid. In Proceedings IEEE Micro Electro Mechanical Systems, pages 71-76, Feb 1993. URL http://dx.doi.org/10. 1109/MEMSYS. 1993.296954.

(83) Q. Wei, K.-D. Li, J. Lian, and L. Wang. Angular dependence of sputtering yield of amorphous and polycrystalline materials. Journal of Physics D: Applied Physics, 41(17):172002, 2008. URL http://dx.doi.org/10.1088/0022-3727/41/17/172002.

(84) G. F. Cerofolini, M. Ferri, E. Romano, F. Suriano, G. P. Veronese, S. Solmi, and D. Narducci. Terascale integration via a redesign of the crossbar based on a vertical arrangement of poly-Si nanowires. Semiconductor Science and Technology, 25(9), 2010. ISSN 02681242. URL https://doi.org/10.1088/0268-1242/25/9/ 095011. 
(85) G. F. Cerofolini, M. Ferri, E. Romano, F. Suriano, G. P. Veronese, S. Solmi, and D. Narducci. Crossbar architecture for tera-scale integration. Semiconductor Science and Technology, 26(4), 2011. ISSN 02681242. URL https://doi.org/10.1088/0268-1242/25/9/ 095011.

(86) N. Burouni, E. Berenschot, M. Elwenspoek, E. Sarajlic, P. Leussink, $\mathrm{H}$. Jansen, and N. Tas. Wafer-scale fabrication of nanoapertures using corner lithography. Nanotechnology, 24(28):285303, 2013. ISSN 1361-6528. URL http://dx.doi.org/10.1088/0957-4484/24/ $28 / 285303$.

(87) X. Yu, H. Zhang, J. K. Oliverio, and P. V. Braun. Template-assisted three-dimensional nanolithography via geometrically irreversible processing. Nano Letters, 9(12):4424-4427, 2009. ISSN 15306984. URL http://dx.doi.org/10.1021/n19027236.

(88) N. Burouni, E. Sarajlic, M. Siekman, L. Abelmann, and N. Tas. Pyramidal nanowire tip for atomic force microscopy and thermal imaging. In 2012 7th IEEE International Conference on Nano/Micro Engineered and Molecular Systems, NEMS 2012, pages 86-89, 2012. ISBN 9781467311243. URL http://dx.doi.org/ 10.1109/NEMS . 2012.6196729.

(89) B. Schurink, J. W. Berenschot, R. M. Tiggelaar, and R. Luttge. Highly uniform sieving structure by corner lithography and silicon wet etching. Microelectronic Engineering, 144:12-18, 2015. ISSN 01679317. URL http://dx.doi.org/10.1016/j . mee.2015.01.027.

(90) M. Malankowska, S. Schlautmann, E. J. W. Berenschot, R. M. Tiggelaar, M. P. Pina, R. Mallada, N. R. Tas, and H. Gardeniers. Three-dimensional fractal geometry for gas permeation in microchannels. Micromachines, 9(2):art. nr. 45, 2018. ISSN 2072666X. URL http://dx.doi.org/10.3390/mi9020045.

(91) B. Wu, A. Kumar, and S. Pamarthy. High aspect ratio silicon etch: A review. Journal of Applied Physics, 108(5), 2010. ISSN 00218979. URL http://dx.doi.org/10.1063/1.3474652.

(92) F. Marty, L. Rousseau, B. Saadany, B. Mercier, O. Français, Y. Mita, and T. Bourouina. Advanced etching of silicon based on deep reactive ion etching for silicon high aspect ratio microstructures 
and three-dimensional micro- and nanostructures. Microelectronics Journal, 36(7):673-677, 2005. ISSN 00262692. URL http: //dx.doi.org/10.1016/j.mejo.2005.04.039.

(93) B. Chang, P. Leussink, F. Jensen, J. Hübner, and H. Jansen. DREM: Infinite etch selectivity and optimized scallop size distribution with conventional photoresists in an adapted multiplexed Bosch DRIE process. Microelectronic Engineering, 191:77 - 83, 2018. ISSN 0167-9317. URL https://doi.org/10.1016/j.mee.2018.01.034.

(94) M. Wang, Y. Yang, J. Shen, J. Jiang, and L. Sun. Visible-lightabsorbing semiconductor/molecular catalyst hybrid photoelectrodes for $\mathrm{H}_{2}$ or $\mathrm{O}_{2}$ evolution: recent advances and challenges. Sustainable Energy Fuels, 1:1641-1663, 2017. URL http://dx.doi. org/10.1039/C7SE00222J.

(95) W. Vijselaar, P. Westerik, J. Veerbeek, R. Tiggelaar, E. Berenschot, N. Tas, H. Gardeniers, and J. Huskens. Spatial decoupling of light absorption and catalytic activity of nickel-molybdenum on highaspect-ratio silicon microwire arrays. Nature Energy, 2018. URL http://dx.doi.org/10.1038/s41560-017-0068-x.

(96) M. G. Walter, E. L. Warren, J. R. McKone, S. W. Boettcher, Q. Mi, E. A. Santori, and N. S. Lewis. Solar water splitting cells. Chemical reviews, $110(11): 6446-6473,2010$.

(97) M. R. Shaner, J. R. McKone, H. B. Gray, and N. S. Lewis. Functional integration of Ni-Mo electrocatalysts with $\mathrm{Si}$ microwire array photocathodes to simultaneously achieve high fill factors and light-limited photocurrent densities for solar-driven hydrogen evolution. Energy Environ. Sci., 8:2977-2984, 2015. URL http://dx.doi.org/10.1039/C5EE01076D.

(98) C. W. Roske, E. J. Popczun, B. Seger, C. G. Read, T. Pedersen, O. Hansen, P. C. K. Vesborg, B. S. Brunschwig, R. E. Schaak, I. Chorkendorff, H. B. Gray, and N. S. Lewis. Comparison of the performance of CoP-coated and Pt-coated radial junction $n+p$ silicon microwire-array photocathodes for the sunlight-driven reduction of water to $\mathrm{H}_{2}(\mathrm{~g})$. The Journal of Physical Chemistry Letters, 6(9):1679-1683, 2015. URL https://doi.org/10.1021/acs . jpclett.5b00495. 
(99) R. H. Coridan, A. C. Nielander, S. A. Francis, M. T. McDowell, V. Dix, S. M. Chatman, and N. S. Lewis. Methods for comparing the performance of energy-conversion systems for use in solar fuels and solar electricity generation. Energy Environ. Sci., 8:2886-2901, 2015. URL http://dx.doi.org/10.1039/C5EE00777A.

(100) C. Battaglia, A. Cuevas, and S. De Wolf. High-efficiency crystalline silicon solar cells: status and perspectives. Energy Environ. Sci., 9:1552-1576, 2016. URL http://dx.doi.org/10.1039/ C5EE03380B.

(101) W. Vijselaar, R. Elbersen, R. M. Tiggelaar, H. Gardeniers, and J. Huskens. Photo-electrical characterization of silicon micropillar arrays with radial $\mathrm{p} / \mathrm{n}$ junctions containing passivation and antireflection coatings. Advanced Energy Materials, 7(7):1601497n/a, 2017. ISSN 1614-6840. URL http://dx.doi.org/10.1002/aenm. 201601497. 1601497.

(102) Y. Chen, K. Sun, H. Audesirk, C. Xiang, and N. S. Lewis. A quantitative analysis of the efficiency of solar-driven water-splitting device designs based on tandem photoabsorbers patterned with islands of metallic electrocatalysts. Energy Environ. Sci., 8:17361747, 2015. URL http://dx.doi.org/10.1039/C5EE00311C.

(103) R. Elbersen, W. Vijselaar, R. M. Tiggelaar, H. Gardeniers, and J. Huskens. Effects of pillar height and junction depth on the performance of radially doped silicon pillar arrays for solar energy applications. Advanced Energy Materials, 6(3):1501728n/a, 2016. ISSN 1614-6840. URL http://dx.doi.org/10.1002/aenm. 201501728. 1501728.

(104) Nickel Institute. Nickel Plating Handbook. 2014. URL https://www.nickelinstitute.org/MediaCentre/Publications/ NickelPlatingHandbook. aspx.

(105) J. R. Mckone, E. L. Warren, M. J. Bierman, S. W. Boettcher, B. S. Brunschwig, N. S. Lewis, and H. B. Gray. Evaluation of Pt, Ni, and $\mathrm{Ni}-\mathrm{Mo}$ electrocatalysts for hydrogen evolution on crystalline Si electrodes. Energy Environ. Sci., 4:3573-3583, 2011. URL http: //dx.doi.org/10.1039/C1EE01488A. 
(106) J. P. Hoare. Boric acid as a catalyst in nickel plating solutions. Journal of The Electrochemical Society, 134(12):3102-3103, 1987. URL http://dx.doi.org/10.1149/1.2100348.

(107) C. Fan, D. L. Piron, A. Sleb, and P. Paradis. Study of electrodeposited nickel-molybdenum, nickel-tungsten, cobaltmolybdenum, and cobalt-tungsten as hydrogen electrodes in alkaline water electrolysis. Journal of The Electrochemical Society, 141(2):382-387, 1994. URL https://doi.org/10.1149/1.2054736.

(108) E. J. Podlaha and D. Landolt. Induced codeposition: I. an experimental investigation of Ni-Mo alloys. Journal of The Electrochemical Society, 143(3):885-892, 1996. URL http://dx.doi.org/ 10.1149/1.1836553.

(109) E. J. Podlaha and D. Landolt. Induced codeposition: II. a mathematical model describing the electrodeposition of Ni-Mo alloys. Journal of The Electrochemical Society, 143(3):893-899, 1996. URL http://dx.doi.org/10.1149/1.1836554.

(110) E. J. Podlaha and D. Landolt. Induced codeposition: III. molybdenum alloys with nickel, cobalt, and iron. Journal of The Electrochemical Society, 144(5):1672-1680, 1997. URL http://dx.doi. org/10.1149/1.1837658.

(111) Y. Chen, J. Shen, and N. xian Chen. The effect of Mo atoms in ternary nitrides with $\eta$-type structure. Solid State Communications, 149(3): 121 - 125, 2009. ISSN 0038-1098. URL https: //doi.org/10.1016/j.ssc.2008.11.004.

(112) Y. Mishima, S. Ochiai, and T. Suzuki. Lattice parameters of $\mathrm{Ni}(\gamma)$, $\mathrm{Ni}_{3} \mathrm{Al}\left(\gamma^{\prime}\right)$ and $\mathrm{Ni}_{3} \mathrm{Ga}\left(\gamma^{\prime}\right)$ solid solutions with additions of transition and B-subgroup elements. Acta Metallurgica, 33(6):1161 - 1169. 1985. ISSN 0001-6160. URL https://doi.org/10.1016/ $0001-6160$ (85) $90211-1$.

(113) O. Schultz, A. Mette, M. Hermle, and S. W. Glunz. Thermal oxidation for crystalline silicon solar cells exceeding 19efficiency applying industrially feasible process technology. Progress in Photovoltaics: Research and Applications, 16(4):317-324, 2008. ISSN 1099-159X. URL http://dx.doi.org/10.1002/pip.814. 
(114) J. Benick, K. Zimmermann, J. Spiegelman, M. Hermle, and S. W. Glunz. Rear side passivation of perc-type solar cells by wet oxides grown from purified steam. Progress in Photovoltaics: Research and Applications, 19(3):361-365, 201 1. ISSN 1099-159X. URL http: //dx.doi.org/10.1002/pip.1020.

(115) S. Mack, A. Wolf, A. Walczak, B. Thaidigsmann, E. A. Wotke, J. J. Spiegelman, R. Preu, and D. Biro. Properties of purified direct steam grown silicon thermal oxides. Solar Energy Materials and Solar Cells, 95(9):2570 - 2575, 2011. ISSN 0927-0248. URL https: //doi.org/10.1016/j. solmat.2011.03.002.

(116) A. T. Garcia-Esparza and K. Takanabe. A simplified theoretical guideline for overall water splitting using photocatalyst particles. J. Mater. Chem. A, 4:2894-2908, 2016. URL http://dx.doi.org/ 10.1039/C5TA06983A.

(117) J. R. McKone, B. F. Sadtler, C. A. Werlang, N. S. Lewis, and H. B. Gray. Ni-Mo nanopowders for efficient electrochemical hydrogen evolution. ACS Catalysis, 3(2):166-169, 2013. URL https: //doi.org/10.1021/cs300691.

(118) M. A. Green. Accuracy of analytical expressions for solar cell fill factors. Solar Cells, 7(3):337 - 340, 1982. ISSN 0379-6787. URL https ://doi.org/10.1016/0379-6787 (82)90057-6.

(119) S. W. Boettcher, E. L. Warren, M. C. Putnam, E. A. Santori, D. TurnerEvans, M. D. Kelzenberg, M. G. Walter, J. R. McKone, B. S. Brunschwig, H. A. Atwater, and N. S. Lewis. Photoelectrochemical hydrogen evolution using Si microwire arrays. Journal of the American Chemical Society, 133(5):1216-1219, 2011. URL https://doi.org/10.1021/ja108801m.

(120) Z. Huang, Z. Chen, Z. Chen, C. LV, H. Meng, and C. Zhang. $\mathrm{Ni}_{12} \mathrm{P}_{5}$ nanoparticles as an efficient catalyst for hydrogen generation via electrolysis and photoelectrolysis. ACS Nano, 8(8):8121-8129, 2014. URL https://doi.org/10.1021/nn5022204.

(121) X.-Q. Bao, M. Fatima Cerqueira, P. Alpuim, and L. Liu. Silicon nanowire arrays coupled with cobalt phosphide spheres as low-cost photocathodes for efficient solar hydrogen evolution. Chem. Commun., 51:10742-10745, 2015. URL http://dx.doi.org/ 10.1039/C5CC02331A. 
(122) C. Lv, Z. Chen, Z. Chen, B. Zhang, Y. Qin, Z. Huang, and C. Zhang. Silicon nanowires loaded with iron phosphide for effective solardriven hydrogen production. J. Mater. Chem. A, 3:17669-17675, 2015. URL http://dx.doi.org/10.1039/C5TA03438H.

(123) L. Veldhuizen, W. Vijselaar, H. Gatz, J. Huskens, and R. Schropp. Textured and micropillar silicon heterojunction solar cells with hotwire deposited passivation layers. Thin Solid Films, 635:66 - 72, 2017. ISSN 0040-6090. URL https://doi.org/10.1016/j.tsf . 2017. 02.048.

(124) G. M. Hale and M. R. Querry. Optical constants of water in the 200-nm to 200- $\mu$ m wavelength region. Appl. Opt., 12(3):555-563, Mar 1973. URL http://dx.doi.org/10.1364/A0.12.000555.

(125) A. G. Tamirat, J. Rick, A. A. Dubale, W.-N. Su, and B.-J. Hwang. Using hematite for photoelectrochemical water splitting: a review of current progress and challenges. Nanoscale Horiz., 1:243-267, 2016. URL http://dx.doi.org/10.1039/C5NH00098J.

(126) X. Zong, S. Thaweesak, H. Xu, Z. Xing, J. Zou, G. M. Lu, and L. Wang. A scalable colloidal approach to prepare hematite films for efficient solar water splitting. Phys. Chem. Chem. Phys., 15:12314-12321, 2013. URL http://dx.doi.org/10.1039/ C3CP52153B.

(127) N. Dalle Carbonare, S. Carli, R. Argazzi, M. Orlandi, N. Bazzanella, A. Miotello, S. Caramori, and C. A. Bignozzi. Improvement of the electron collection efficiency in porous hematite using a thin iron oxide underlayer: towards efficient all-iron based photoelectrodes. Phys. Chem. Chem. Phys., 17:29661-29670, 2015. URL http://dx.doi.org/10.1039/C5CP04152J.

(128) X. Chen, M. Wang, J. Chen, and S. Shen. Electrophoretic deposition of nanostructured hematite photoanodes for solar hydrogen generation. Journal of Materials Research, 31(11):1547???1553, 2016. URL http://dx.doi.org/10.1557/jmr.2016.37.

(129) N. Dalle Carbonare, R. Boaretto, S. Caramori, R. Argazzi, M. Dal Colle, L. Pasquini, R. Bertoncello, M. Marelli, C. Evangelisti, and C. A. Bignozzi. Photoelectrochemical behavior of electrophoretically deposited hematite thin films modified with ti(iv). 
Molecules, 21(7), 2016. ISSN 1420-3049. URL https://doi.org/10. $3390 /$ molecules21070942.

(130) L. Xi, S. Y. Chiam, W. F. Mak, P. D. Tran, J. Barber, S. C. J. Loo, and L. H. Wong. A novel strategy for surface treatment on hematite photoanode for efficient water oxidation. Chem. Sci., 4: 164-169, 2013. URL http://dx.doi.org/10.1039/C2SC20881D.

(131) Gurudayal, R. A. John, P. P. Boix, C. Yi, C. Shi, M. C. Scott, S. A. Veldhuis, A. M. Minor, S. M. Zakeeruddin, L. H. Wong, M. Grätzel, and N. Mathews. Atomically altered hematite for highly efficient perovskite tandem water-splitting devices. ChemSusChem, 10 (11):2449-2456, 2017. ISSN 1864-564X. URL http://dx.doi.org/ $10.1002 / \operatorname{cssc} .201700159$.

(132) L. Vayssieres, N. Beermann, S.-E. Lindquist, , and A. Hagfeldt. Controlled aqueous chemical growth of oriented three-dimensional crystalline nanorod arrays: Application to iron(III) oxides. Chemistry of Materials, 13(2):233-235, 2001. URL http://dx.doi.org/10. $1021 / \mathrm{cm} 001202 \mathrm{x}$.

(133) J. A. Glasscock, P. R. F. Barnes, I. C. Plumb, and N. Savvides. Enhancement of photoelectrochemical hydrogen production from hematite thin films by the introduction of $\mathrm{Ti}$ and $\mathrm{Si}$. Journal of Physical Chemistry C, 111(44):16477-16488, 2007. URL http://dx.doi.org/10.1021/jp0745561.

(134) C. D. Bohn, A. K. Agrawal, E. C. Walter, M. D. Vaudin, A. A. Herzing, P. M. Haney, A. A. Talin, and V. A. Szalai. Effect of tin doping on $\alpha-\mathrm{Fe}_{2} \mathrm{O}_{3}$ photoanodes for water splitting. The Journal of Physical Chemistry C, 116(29): 15290-15296, 2012. URL http://dx.doi.org/ 10.1021/jp305221v.

(135) D. Yan, J. Tao, K. Kisslinger, J. Cen, Q. Wu, A. Orlov, and M. Liu. The role of the domain size and titanium dopant in nanocrystalline hematite thin films for water photolysis. Nanoscale, 7: 18515-18523, 2015. URL http://dx.doi.org/10.1039/C5NR05894E.

(136) W. Liu, H. Liu, L. Dang, H. Zhang, X. Wu, B. Yang, Z. Li, $X$. Zhang, L. Lei, and S. Jin. Amorphous cobalt-iron hydroxide nanosheet electrocatalyst for efficient electrochemical and photo-electrochemical oxygen evolution. Advanced Functional 
Materials, 27(14):1603904-n/a, 2017. ISSN 1616-3028. URL http: //dx.doi.org/10.1002/adfm.201603904. 1603904.

(137) M. Descostes, F. Mercier, N. Thromat, C. Beaucaire, and M. Gautier-Soyer. Use of xps in the determination of chemical environment and oxidation state of iron and sulfur samples: constitution of a data basis in binding energies for Fe and $\mathrm{S}$ reference compounds and applications to the evidence of surface species of an oxidized pyrite in a carbonate medium. Applied Surface Science, 165(4):288 - 302, 2000. ISSN 0169-4332. URL https://doi.org/10.1016/S0169-4332(00)00443-8.

(138) B. Seger, I. Castelli, P. Vesborg, K. Jacobsen, O. Hansen, and I. Chorkendorff. 2-photon tandem device for water splitting: Comparing photocathode first versus photoanode first designs. Energy and Environmental Science, 7(8):2397-2413, 2014. URL http://dx.doi.org/10.1039/c4ee01335b.

(139) M. Mayer, C. Du, and D. Wang. Hematite/Si nanowire dualabsorber system for photoelectrochemical water splitting at low applied potentials. Journal of the American Chemical Society, 134(30):12406-12409, 2012. URL http://dx.doi.org/10.1021/ ja3051734.

(140) R. van de Krol and Y. Liang. An $\mathrm{n}-\mathrm{Si} / \mathrm{n}-\mathrm{Fe}_{2} \mathrm{O}_{3}$ heterojunction tandem photoanode for solar water splitting. CHIMIA International Journal for Chemistry, 67(3): 168-171, 2013. ISSN 0009-4293. URL http://dx.doi.org/10.2533/chimia.2013.168.

(141) X. Qi, G. She, X. Huang, T. Zhang, H. Wang, L. Mu, and W. Shi. Highperformance $\mathrm{n}-\mathrm{Si} / \alpha-\mathrm{Fe}_{2} \mathrm{O}_{3}$ core/shell nanowire array photoanode towards photoelectrochemical water splitting. Nanoscale, 6:3182-3189, 2014. URL http://dx.doi.org/10.1039/C3NR05429B.

(142) S. Ramadurgam, T.-G. Lin, and C. Yang. Aluminum plasmonics for enhanced visible light absorption and high efficiency water splitting in core-multishell nanowire photoelectrodes with ultrathin hematite shells. Nano Letters, 14(8):4517-4522, 2014. URL http://dx.doi.org/10.1021/n1501541s.

(143) K. Jun, Y. S. Lee, T. Buonassisi, and J. M. Jacobson. High photocurrent in silicon photoanodes catalyzed by iron oxide thin films for 
water oxidation. Angewandte Chemie International Edition, 51 (2):423-427, 2012. ISSN 1521-3773. URL http://dx.doi.org/10. 1002/anie. 201104367.

(144) R. D. L. Smith, M. S. Prévot, R. D. Fagan, Z. Zhang, P. A. Sedach, M. K. J. Siu, S. Trudel, and C. P. Berlinguette. Photochemical route for accessing amorphous metal oxide materials for water oxidation catalysis. Science, 340(6128):60-63, 2013. ISSN 0036-8075. URL http://dx.doi.org/10.1126/science.1233638.

(145) B. Chung, G. F. Virshup, S. Hikido, and N. R. Kaminar. $276 \%$ efficiency (1 sun, air mass 1.5) monolithic $\mathrm{Al}_{0.37} \mathrm{Ga}_{0.63} \mathrm{As} / \mathrm{GaAs}$ twojunction cascade solar cell with prismatic cover glass. Applied Physics Letters, 55(17):1741-1743, 1989. URL https://doi.org/10. 1063/1.102204.

(146) S. R. Kurtz and R. G. Gordon. Transparent conducting electrodes on silicon. Solar Energy Materials, 15(4):229 - 236, 1987. ISSN 01651633. URL http://dx.doi.org/10.1016/0165-1633(87)90038-4.

(147) J. H. Noh, S. Lee, J. Y. Kim, J.-K. Lee, H. S. Han, C. M. Cho, I. S. Cho, H. S. Jung, and K. S. Hong. Functional multilayered transparent conducting oxide thin films for photovoltaic devices. The Journal of Physical Chemistry C, 113(3):1083-1087, 2009. URL http://dx. doi.org/10.1021/jp808279j.

(148) E. R. Ollotu, M.E.Samiji, and R. Kivaisi. Influence of films thickness on optical properties of $\mathrm{Nb}$-doped $\mathrm{TiO}_{2}$ (NTO) thin films deposited by DC reactive magnetron sputtering. International Journal of Nano Science and Technology, 2(1): 1-10, 2014. URL http://www.ijnst.com/IJNST_Vol. $\% 202, \% 20$ No. $\% 201$, $\% 20$ January $\% 202014 /$ Influence $\% 20$ of $\% 20 \mathrm{Films}$.pdf.

(149) D. Rafieian, R. T. Driessen, W. Ogieglo, and R. G. Lammertink. Intrinsic photocatalytic assessment of reactively sputtered $\mathrm{TiO}_{2}$ films. ACS Applied Materials \& Interfaces, 7(16):8727-8732, 2015. URL http://dx.doi.org/10.1021/acsami.5b01047.

(150) D. Rafieian, W. Ogieglo, T. Savenije, and R. G. H. Lammertink. Controlled formation of anatase and rutile $\mathrm{TiO}_{2}$ thin films by reactive magnetron sputtering. AIP Advances, 5(9):097168, 2015. URL https://doi.org/10.1063/1.4931925. 
(151) M. G. Kast, L. J. Enman, N. J. Gurnon, A. Nadarajah, and S. W. Boettcher. Solution-deposited $\mathrm{F}: \mathrm{SnO}_{2} / \mathrm{TiO}_{2}$ as a base-stable protective layer and antireflective coating for microtextured buriedjunction $\mathrm{H}_{2}$-evolving Si photocathodes. ACS Applied Materials \& Interfaces, 6(24):22830-22837, 2014. URL http://dx.doi.org/10. 1021/am506999p.

(152) M. Taguchi, A. Yano, S. Tohoda, K. Matsuyama, Y. Nakamura, T. Nishiwaki, K. Fujita, and E. Maruyama. $247 \%$ record efficiency HIT solar cell on thin silicon wafer. IEEE Journal of Photovoltaics, 4 (1):96-99, Jan 2014. ISSN 2156-3381.

(153) T. E. McKone, W. W. Nazaroff, P. Berck, M. Auffhammer, T. Lipman, M. S. Torn, E. Masanet, A. Lobscheid, N. Santero, U. Mishra, A. Barrett, M. Bomberg, K. Fingerman, C. Scown, B. Strogen, and A. Horvath. Grand challenges for life-cycle assessment of biofuels. Environmental Science \& Technology, 45(5):1751-1756, 2011. URL http://dx.doi.org/10.1021/es103579c.

(154) K. R. Tolod, S. Hernández, and N. Russo. Recent advances in the $\mathrm{BiVO}_{4}$ photocatalyst for sun-driven water oxidation: Topperforming photoanodes and scale-up challenges. Catalysts, 7(1), 2017. ISSN 2073-4344. URL http://dx.doi.org/10.3390/ catal7010013.

(155) P. Perez-Rodriguez, I. Digdaya, A. M. Raventos, M. Falkenberg, R. Vasudevan, M. Zeman, W. Smith, and A. H. M. Smets. Solar fuel production by using PV/PEC junctions based on earth-abundant materials. In 2016 IEEE 43rd Photovoltaic Specialists Conference (PVSC), pages 3620-3624, June 2016. URL http://dx . doi .org/10. 1109/PVSC. 2016.7750348

(156) Z. Huang, N. Geyer, P. Werner, J. de Boor, and U. Gösele. Metalassisted chemical etching of silicon: A review. Advanced Materials, 23(2):285-308, 2011. ISSN 1521-4095. URL http://dx.doi.org/ 10.1002 /adma. 201001784 .

(157) C. Du, X. Yang, M. T. Mayer, H. Hoyt, J. Xie, G. McMahon, G. Bischoping, and D. Wang. Hematite-based water splitting with low turn-on voltages. Angewandte Chemie International Edition, 52(48): 12692-12695, 2013. ISSN 1521-3773. URL http: //dx.doi.org/10.1002/anie.201306263. 
(158) X. Yang, R. Liu, Y. He, J. Thorne, Z. Zheng, and D. Wang. Enabling practical electrocatalyst-assisted photoelectron-chemical water splitting with earth abundant materials. Nano Research, 8(1): 56-81, Jan 2015. ISSN 1998-0000. URL https://doi.org/10.1007/ s12274-014-0645-2.

(159) G. Merle, M. Wessling, and K. Nijmeijer. Anion exchange membranes for alkaline fuel cells: A review. Journal of Membrane Science, 377(1):1 - 35, 2011. ISSN 0376-7388. URL https://doi. org $/ 10.1016 / j$.memsci.2011.04.043.

(160) S. Ardo, D. Fernandez Rivas, M. Modestino, V. Schulze Greiving, F. F. Abdi, E. Alarcon llado, V. Artero, K. Ayers, C. Battaglia, J.P. Becker, D. Bederak, A. Berger, F. Buda, E. Chinello, B. Dam, V. Di Palma, T. Edvinsson, K. Fujii, H. Gardeniers, H. Geerlings, S. M. H. Hashemi, S. Haussener, F. Houle, J. Huskens, B. D. James, K. Konrad, P. P. Kunturu, D. Lohse, B. Mei, E. L. Miller, G. Moore, J. Muller, K. L. Orchard, T. E. Rosser, F. Saadi, J.-W. Schüttauf, B. Seger, S. W. Sheehan, W. A. Smith, J. Spurgeon, M. Tang, R. v. d. $\mathrm{Krol}, \mathrm{P}$. C. Vesborg, and P. Westerik. Pathways to electrochemical solar-hydrogen technologies. 2018. submitted.

(161) S. Grigoriev, V. Porembskiy, S. Korobtsev, V. Fateev, F. Auprêtre, and P. Millet. High-pressure PEM water electrolysis and corresponding safety issues. International Journal of Hydrogen Energy, 36(3):2721 - 2728, 2011. ISSN 0360-3199. URL https://doi.org/ $10.1016 / j . i j h y d e n e .2010 .03 .058$. The Third Annual International Conference on Hydrogen Safety.

(162) T. Jacobsson, V. Fjällström, M. Edoff, and T. Edvinsson. Sustainable solar hydrogen production: From photoelectrochemical cells to PV-electrolyzers and back again. Energy and Environmental Science, 7(7):2056-2070, 2014. URL http://dx.doi.org/10.1039/ c4ee00754a.

(163) M. A. Modestino and S. Haussener. An integrated device view on photo-electrochemical solar-hydrogen generation. Annual Review of Chemical and Biomolecular Engineering, 6(1):13-34, 2015. URL https://doi.org/10.1146/ annurev-chembioeng-061114-123357. 
(164) A. C. Nielander, M. R. Shaner, K. M. Papadantonakis, S. A. Francis, and N. S. Lewis. A taxonomy for solar fuels generators. Energy Environ. Sci., 8: 16-25, 2015. URL http://dx.doi.org/10.1039/ C4EE02251C.

(165) J. McKone, N. Lewis, and H. Gray. Will solar-driven water-splitting devices see the light of day? Chemistry of Materials, 26(1):407414, 2014. URL http://dx.doi.org/10.1021/cm4021518.

(166) J. Vindel and J. Polo. Intermittency and variability of daily solar irradiation. Atmospheric Research, 143(Supplement C):313 - 327 , 2014. ISSN 0169-8095. URL https://doi.org/10.1016/j.atmosres. 2014.03 .001$.

(167) M. R. Shaner, H. A. Atwater, N. S. Lewis, and E. W. McFarland. A comparative technoeconomic analysis of renewable hydrogen production using solar energy. Energy Environ. Sci., 9:2354-2371, 2016. URL http://dx.doi.org/10.1039/C5EE02573G.

(168) C. Rodriguez, M. Modestino, D. Psaltis, and C. Moser. Design and cost considerations for practical solar-hydrogen generators. Energy and Environmental Science, 7(12):3828-3835, 2014. URL http://dx.doi.org/10.1039/c4ee01453g.

(169) S. W. Sheehan, E. R. Cave, K. P. Kuhl, N. Flanders, A. L. Smeigh, and D. T. Co. Commercializing solar fuels within today's markets. Chem, 3(1):3 - 7, 2017. ISSN 2451-9294. URL https://doi.org/10. 1016/j.chempr. 2017.06.003.

(170) N. G. Kulkarni and V. B. Virulkar. Power electronics and its application to solar photovoltaic systems in india. Energy and Power Engineering, 8(2):76-91, 2016. URL http://dx.doi.org/10.4236/ epe. 2016.82007 .

(171) F. C. T. Office. Multi-year research, development, and demonstration plan, $2012 . \quad$ URL https://energy.gov/eere/fuelcells/downloads/ fuel-cell-technologies-office-multi-year-research-development-and-22.

(172) U.S. DRIVE. Hydrogen production technical team roadmap, 2013. URL https://energy.gov/eere/vehicles/downloads/ us-drive-hydrogen-production-technical-team-roadmap. 
(173) N. M. Haegel, R. Margolis, T. Buonassisi, D. Feldman, A. Froitzheim, R. Garabedian, M. Green, S. Glunz, H.-M. Henning, B. Holder, I. Kaizuka, B. Kroposki, K. Matsubara, S. Niki, K. Sakurai, R. A. Schindler, W. Tumas, E. R. Weber, G. Wilson, M. Woodhouse, and S. Kurtz. Terawatt-scale photovoltaics: Trajectories and challenges. Science, 356(6334):141-143, 2017. ISSN 0036-8075. URL http://dx.doi.org/10.1126/science.aal1288.

(174) J. Jia, L. C. Seitz, J. D. Benck, Y. Huo, Y. Chen, J. W. D. Ng, T. Bilir, J. S. Harris, and T. F. Jaramillo. Solar water splitting by photovoltaicelectrolysis with a solar-to-hydrogen efficiency over 30\%. Nature Communications, 7, 2016. URL http://dx.doi.org/10.1038/ ncomms 13237.

(175) W. J. Chang, K.-H. Lee, H. Ha, K. Jin, G. Kim, S.-T. Hwang, H.-m. Lee, S.-W. Ahn, W. Yoon, H. Seo, J. S. Hong, Y. K. Go, J.-I. Ha, and K. T. Nam. Design principle and loss engineering for photovoltaicelectrolysis cell system. ACS Omega, 2(3): 1009-1018, 2017. URL http://dx.doi.org/10.1021/acsomega.7b00012.

(176) N. Guillet and P. Millet. Alkaline Water Electrolysis, pages 117-166. Wiley-VCH Verlag GmbH \& Co. KGaA, 2015. ISBN 9783527676507. URL http://dx.doi.org/10.1002/9783527676507.ch4.

(177) S. Haussener, C. Xiang, J. Spurgeon, S. Ardo, N. Lewis, and A. Weber. Modeling, simulation, and design criteria for photoelectrochemical water-splitting systems. Energy and Environmental Science, 5(12):9922-9935, 2012. URL http://dx.doi.org/10.1039/ c2ee23187e.

(178) M. A. Modestino, K. A. Walczak, A. Berger, C. M. Evans, S. Haussener, C. Koval, J. S. Newman, J. W. Ager, and R. A. Segalman. Robust production of purified h2 in a stable, selfregulating, and continuously operating solar fuel generator. Energy Environ. Sci., 7:297-301, 2014. URL http://dx.doi.org/10. 1039/C3EE43214A.

(179) J.-W. Schüttauf, M. A. Modestino, E. Chinello, D. Lambelet, A. Delfino, D. Dominé, A. Faes, M. Despeisse, J. Bailat, D. Psaltis, C. Moser, and C. Ballif. Solar-to-hydrogen production at $14.2 \%$ efficiency with silicon photovoltaics and earth-abundant electrocatalysts. Journal of The Electrochemical Society, 163(10):F1177F1181, 2016. URL http://dx.doi.org/10.1149/2.0541610jes. 
(180) J. W. Ager, M. R. Shaner, K. A. Walczak, I. D. Sharp, and S. Ardo. Experimental demonstrations of spontaneous, solar-driven photoelectrochemical water splitting. Energy Environ. Sci., 8:28112824, 2015. URL http://dx.doi.org/10.1039/C5EE00457H.

(181) R. Sathre, J. B. Greenblatt, K. Walczak, I. D. Sharp, J. C. Stevens, J. W. Ager, and F. A. Houle. Opportunities to improve the net energy performance of photoelectrochemical water-splitting technology. Energy Environ. Sci., 9:803-819, 2016. URL http://dx.doi. org/10.1039/C5EE03040D.

(182) P. van der Linde, A. Moreno Soto, P. Peñas López, J. RodríguezRodríguez, D. Lohse, H. Gardeniers, D. van der Meer, and D. Fernández Rivas. Electrolysis-driven and pressure-controlled diffusive growth of successive bubbles on microstructured surfaces. Langmuir, 33(45): 12873-12886, 2017. URL http://dx.doi.org/10.1021/ acs. langmuir.7b0297.

(183) S. Haussener, S. Hu, C. Xiang, A. Z. Weber, and N. S. Lewis. Simulations of the irradiation and temperature dependence of the efficiency of tandem photoelectrochemical water-splitting systems. Energy Environ. Sci., 6:3605-3618, 2013. URL http://dx.doi.org/ 10.1039/C3EE41302K.

(184) S. Hu, C. Xiang, S. Haussener, A. D. Berger, and N. S. Lewis. An analysis of the optimal band gaps of light absorbers in integrated tandem photoelectrochemical water-splitting systems. Energy Environ. Sci., 6:2984-2993, 2013. URL http://dx.doi.org/10.1039/ C3EE40453F.

(185) R. Sathre, C. D. Scown, W. R. Morrow, J. C. Stevens, I. D. Sharp, J. W. Ager, K. Walczak, F. A. Houle, and J. B. Greenblatt. Lifecycle net energy assessment of large-scale hydrogen production via photoelectrochemical water splitting. Energy Environ. Sci., 7: 3264-3278, 2014. URL http://dx.doi.org/10.1039/C4EE01019A.

(186) L. C. Seitz, Z. Chen, A. J. Forman, B. A. Pinaud, J. D. Benck, and T. F. Jaramillo. Modeling practical performance limits of photoelectrochemical water splitting based on the current state of materials research. ChemSusChem, 7(5):1372-1385, 2014. ISSN 1864564X. URL http://dx.doi.org/10.1002/cssc.201301030. 
(187) M. Dumortier, S. Tembhurne, and S. Haussener. Holistic design guidelines for solar hydrogen production by photoelectrochemical routes. Energy Environ. Sci., 8:3614-3628, 2015. URL http://dx.doi.org/10.1039/C5EE01821H.

(188) M. Dumortier and S. Haussener. Design guidelines for concentrated photo-electrochemical water splitting devices based on energy and greenhouse gas yield ratios. Energy Environ. Sci., 8: 3069-3082, 2015. URL http://dx.doi.org/10.1039/C5EE01269D.

(189) M. A. Modestino, S. M. H. Hashemi, and S. Haussener. Mass transport aspects of electrochemical solar-hydrogen generation. Energy Environ. Sci., 9:1533-1551, 2016. URL http://dx.doi.org/10. 1039/C5EE03698D.

(190) C. Xiang, A. Z. Weber, S. Ardo, A. Berger, Y. Chen, R. Coridan, K. T. Fountaine, S. Haussener, S. Hu, R. Liu, N. S. Lewis, M. A. Modestino, M. M. Shaner, M. R. Singh, J. C. Stevens, K. Sun, and K. Walczak. Modeling, simulation, and implementation of solar-driven watersplitting devices. Angewandte Chemie International Edition, 55 (42): 12974-12988, 2016. ISSN 1521-3773. URL http://dx.doi.org/ 10.1002/anie. 201510463.

(191) B. A. Pinaud, J. D. Benck, L. C. Seitz, A. J. Forman, Z. Chen, T. G. Deutsch, B. D. James, K. N. Baum, G. N. Baum, S. Ardo, H. Wang, E. Miller, and T. F. Jaramillo. Technical and economic feasibility of centralized facilities for solar hydrogen production via photocatalysis and photoelectrochemistry. Energy Environ. Sci., 6: 19832002, 2013. URL http://dx.doi.org/10.1039/C3EE40831K.

(192) D. M. Fabian, S. Hu, N. Singh, F. A. Houle, T. Hisatomi, K. Domen, F. E. Osterloh, and S. Ardo. Particle suspension reactors and materials for solar-driven water splitting. Energy Environ. Sci., 8:28252850, 2015. URL http://dx.doi.org/10.1039/C5EE01434D.

(193) Q. Wang, T. Hisatomi, Q. Jia, H. Tokudome, M. Zhong, C. Wang, Z. Pan, T. Takata, M. Nakabayashi, N. Shibata, Y. Li, I. D. Sharp, A. Kudo, T. Yamada, and K. Domen. Scalable water splitting on particulate photocatalyst sheets with a solar-to-hydrogen energy conversion efficiency exceeding 1\%. Nature Materials, 15(6):611615, 2016. URL http://dx.doi.org/10.1038/nmat4589. 
(194) K. Maeda, K. Teramura, H. Masuda, T. Takata, N. Saito, Y. Inoue, and $\mathrm{K}$. Domen. Efficient overall water splitting under visible-light irradiation on $\left(\mathrm{Ga}_{1}-\mathrm{x} Z \mathrm{n}_{\mathrm{x}}\right)\left(\mathrm{N}_{1}-\mathrm{x} \mathrm{O}_{\mathrm{x}}\right)$ dispersed with $\mathrm{Rh}-\mathrm{Cr}$ mixedoxide nanoparticles: Effect of reaction conditions on photocatalytic activity. The Journal of Physical Chemistry B, 110(26): 1310713112, 2006. URL http://dx.doi.org/10.1021/jp0616563.

(195) N. Kaeffer, A. Morozan, and V. Artero. Oxygen tolerance of a molecular engineered cathode for hydrogen evolution based on a cobalt diimine-dioxime catalyst. The Journal of Physical Chemistry B, 119(43): 13707-13713, 2015. URL http://dx.doi.org/ 10.1021/acs.jpcb.5b03136.

(196) D. W. Wakerley and E. Reisner. Oxygen-tolerant proton reduction catalysis: much $\mathrm{O}_{2}$ about nothing? Energy Environ. Sci., 8:22832295, 2015. URL http://dx.doi.org/10.1039/C5EE01167A.

(197) R. Chaubey, S. Sahu, O. O. James, and S. Maity. A review on development of industrial processes and emerging techniques for production of hydrogen from renewable and sustainable sources. Renewable and Sustainable Energy Reviews, 23 (Supplement C):443 - 462, 2013. ISSN 1364-0321. URL https: //doi.org/10.1016/j.rser.2013.02.019.

(198) C. Seidel. The application of life cycle assessment to public policy development. The International Journal of Life Cycle Assessment, 21(3):337-348, Mar 2016. ISSN 1614-7502. URL https: //doi.org/10.1007/s11367-015-1024-2.

(199) P. Vesborg and T. Jaramillo. Addressing the terawatt challenge: Scalability in the supply of chemical elements for renewable energy. RSC Advances, 2(21):7933-7947, 2012. ISSN 2046-2069. URL https://doi.org/10.1039/c2ra20839c.

(200) E. W. McFarland. Solar energy: setting the economic bar from the top-down. Energy Environ. Sci., 7:846-854, 2014. URL http: //dx.doi.org/10.1039/C3EE43714K.

(201) E. Kemppainen, A. Bodin, B. Sebok, T. Pedersen, B. Seger, B. Mei, D. Bae, P. C. K. Vesborg, J. Halme, O. Hansen, P. D. Lund, and I. Chorkendorff. Scalability and feasibility of photoelectrochemical $\mathrm{H}_{2}$ evolution: the ultimate limit of $\mathrm{Pt}$ nanoparticle as an 
HER catalyst. Energy Environ. Sci., 8:2991-2999, 2015. URL http: //dx.doi.org/10.1039/C5EE02188J.

(202) B. Mei, G. Mul, and B. Seger. Beyond water splitting: Efficiencies of photo-electrochemical devices producing hydrogen and valuable oxidation products. Advanced Sustainable Systems, 1(1-2): 1600035, 2017. ISSN 2366-7486. URL http://dx.doi.org/10.1002/ adsu. 201600035.

(203) C. Chen, A. J. Bloomfield, and S. W. Sheehan. Selective electrochemical oxidation of lactic acid using iridium-based catalysts. Industrial \& Engineering Chemistry Research, 56(13):3560-3567, 2017. URL http://dx.doi.org/10.1021/acs.iecr.6b05073.

(204) D. Pletcher and F. Walsh. Industrial Electrochemistry. Springer Netherlands, 2012. ISBN 978-0-7514-0148-6. URL http://www. springer.com/la/book/9780751401486.

(205) G. P. Peters, R. M. Andrew, J. G. Canadell, S. Fuss, R. B. Jackson, J. I. Korsbakken, C. Le Quéré, and N. Nakicenovic. Key indicators to track current progress and future ambition of the paris agreement. Nature Climate Change, 7(2):118-122, 2017. URL http://dx.doi.org/10.1038/nclimate3202. Cited By :16.

(206) A. Hof, C. Brink, A. M. Beltran, and M. den Elzen. Greenhouse gas emission reduction targets for 2030, 12 2012. URL http://www.pbl.nl/en/publications/2012/ greenhouse-gas-emission-reduction-targets-for-2030. accessed: 09-01-2018.

(207) S. Cherryman, S. King, F. Hawkes, R. Dinsdale, and D. Hawkes. An exploratory study of public opinions on the use of hydrogen energy in Wales. Public Understanding of Science, 17(3):397-410, 2008. URL https://doi.org/10.1177/0963662506068053.

(208) S. Bakker, H. van Lente, and R. Engels. Competition in a technological niche: the cars of the future. Technology Analysis \& Strategic Management, 24(5):421-434, 2012. URL https://doi. org/10.1080/09537325.2012.674666.

(209) N. Melton, J. Axsen, and D. Sperling. Moving beyond alternative fuel hype to decarbonize transportation. Nature Energy, 1:16013, 2016. URL https://doi.org/10.1038/nenergy . 2016.13. 
(210) W. E. Council. World energy scenarios: Global transport scenarios 2050, 2011. URL https://www .worldenergy.org/publications/ 2011/global-transport-scenarios-2050/. accessed: 09-01-2018.

(211) T. A. Faunce, W. Lubitz, A. W. B. Rutherford, D. MacFarlane, G. F. Moore, P. Yang, D. G. Nocera, T. A. Moore, D. H. Gregory, S. Fukuzumi, K. B. Yoon, F. A. Armstrong, M. R. Wasielewski, and S. Styring. Energy and environment policy case for a global project on artificial photosynthesis. Energy Environ. Sci., 6:695698, 2013. URL http://dx.doi.org/10.1039/C3EE00063J.

(212) T. Faunce, S. Styring, M. R. Wasielewski, G. W. Brudvig, A. W. Rutherford, J. Messinger, A. F. Lee, C. L. Hill, H. deGroot, M. Fontecave, D. R. MacFarlane, B. Hankamer, D. G. Nocera, D. M. Tiede, H. Dau, W. Hillier, L. Wang, and R. Amal. Artificial photosynthesis as a frontier technology for energy sustainability. Energy Environ. Sci., 6:1074-1076, 2013. URL http://dx.doi.org/10.1039/ C3EE40534F. 


\section{Summary}

This thesis has described the usefulness of some microfabrication techniques for the development of solar-to-hydrogen devices. Solar-to-hydrogen devices are being developed as a way to deal with the intermittent nature of solar energy, to generate a fuel which can be stored, instead of electricity which has to be used directly when it is generated. The voltage and current produced by these solar-tohydrogen devices is used to electrochemically split water into oxygen and the fuel hydrogen. However the combined management of photons, electrons, ions and bubbles in such devices is challenging and often leads to trade-offs and sub-optimal efficiencies. The challenge is to find device architectures that can circumvent these trade-offs as much as possible, especially by finding the right combination and arrangement of semiconductor materials, making sure that catalysts and gas separators do not interfere with light absorption, and taking care of good ion conduction without reducing the illuminated semiconductor area or creating explosive product streams.

Architectures with micrometer-sized features can help to overcome some of these trade-offs. Microfabrication specializes in the parallel fabrication of numerous micro and nanostructures on silicon wafers, and silicon has proven its value for photovoltaics and is also investigated for solar water splitting devices. However the control that these microfabrication techniques give over silicon structures in the out-ofplane direction is limited. Therefore some new techniques were developed for patterning the sidewalls of silicon structures. A special inclined ion beam etching technique was developed for selective etching of horizontal surfaces, and a special Bosch etching recipe for creating multiple semicircular features along vertical silicon structures. Combined with Retraction Edge Lithography and Corner Lithography, se- 
lective opening of the top or bottom or one or several bands along the sidewall of such structures was achieved. The technology is accurate and can produce wafer-scale arrays of the same 3D monocrystaline structures in parallel.

The selective opening of the top part of a silicon structure was applied to a silicon microwire photocathode with a radial $n^{+} / p$-junction in the wires, to achieve spatioselective deposition of a nickel-molybdenum alloy, an opaque catalyst made of only earth-abundant elements. In this way the amount of catalyst-covered and illuminated surface area could be optimized separately, to avoid both the catalysislimited and light absorption-limited regimes in the parameter space, and reach an efficiency of $10.8 \%$, a record for silicon wire-based photocathodes.

First steps towards a fully integrated solar-to-hydrogen device were made. Based on band gaps, available catalysts and material stabilities, it was investigated whether a photoabsorber tandem configuration with silicon and hematite could be made fully integrated and used for providing all the necessary current and voltage for splitting water. A $\mathrm{Ti}_{99} \mathrm{Nb}_{1}$ connection layer was developed, and tandem operation was shown for the combination of a monocrystaline silicon buried junction solar cell with a reactively sputtered hematite photoanode. However the performance of the tandem device was poor, with the onset of water oxidation around $0.5 \mathrm{~V}$ vs RHE and a photocurrent around $40 \mu \mathrm{Acm}^{-2}$ at $1.23 \mathrm{~V}$ vs RHE. There are certainly promising possibilities for improving the performance, but it remains uncertain if such tandem devices can ever compete with silicon-based triple cells as the driving force for water electrolysis.

Another step towards a fully integrated solar-to-hydrogen device was the investigation of an innovative device concept based on a silicon membrane structure with micropores. The membrane has integrated electrodes on both sides, and can split water with ion transport losses below $100 \mathrm{mV}$ at a current density of $10 \mathrm{~mA} \mathrm{~cm}^{-2}$ in a $1 \mathrm{M} \mathrm{NaOH}$ electrolyte. It can do this while around $99 \%$ of the surface area of the device remains available for light absorption. Without use of an ionselective membrane, hydrogen crossover to the oxygen generating compartment was around $1 \%$. This shows the promise of the device architecture, as it circumvents the trade-offs between ion transport, light absorption and gas separation.

Several device architectures were considered, and it was argued 
that all devices that use photoelectrodes made out of earth-abundant elements need some form of microstructuring to circumvent certain trade-offs. These materials can often be processed with cheap, solutionbased methods, and if their performance can be improved they hold great promise for solar hydrogen with a reasonable price. However state-of-the-art silicon-based triple cells can currently drive the water splitting reaction more efficiently, and require less integration with the 'electrolyzer' part of the solar-to-hydrogen device because they do not depend on a semiconductor-liquid junction. Therefore trade-offs can be more easily avoided (without microstructuring) and manufacturing could be easier. Economically it is probably even more favorable to completely separate the solar cells and the electrolyzer, and only connect them with an electrical wire (and potentially power electronics). This would allow separate optimization of both components. If the installation is additionally connected to the power grid, the electricity could also be used directly when the demand is high enough, thereby avoiding the losses associated with both the water splitting and recombination reactions.

At least for the near future, the implementation of such 'PV-Electrolysis' seems more likely than the use of the more integrated photoelectrochemical devices. Because of the low price of fossil fuel-based energy and hydrogen, first applications will probably only be feasible for small, grid-independent installations or perhaps for the generation of hydrogen as a chemical feedstock. However advancements in photoelectrode materials, catalysts and device architectures (including the necessary microstructuring), as well as the depletion of fossil fuels and the implementation of taxes and subsidies that favor the use of renewable energy, could make solar hydrogen interesting for the global energy market in the long term. 


\section{Samenvatting}

In dit proefschrift is de bruikbaarheid van enkele microfabricagetechnieken besproken voor toepassing in panelen die waterstof maken door gebruik te maken van zonne-energie. Zulke zogenaamde solar-tohydrogen devices worden ontwikkeld in de hoop dat daarmee beter omgegaan kan worden met de onregelmatigheid van zonne-energie, door een brandstof te maken die kan worden opgeslagen, in plaats van elektriciteit die direct gebruikt moet worden als het opgewekt is. Het voltage en de stroom die door deze solar-to-hydrogen devices geleverd worden worden gebruikt om elektrochemisch water te splitsen in zuurstof en de brandstof waterstof. Om daarbij tegelijk goed om te gaan met fotonen, elektronen, ionen en bubbels is een uitdaging waarbij er vaak een compromis gevonden moet worden met een niet optimale efficiëntie. De uitdaging is dan om het device zo te ontwerpen dat er zo min mogelijk compromissen nodig zijn, met name door de juiste combinatie en configuratie van halfgeleidermaterialen te vinden, door te zorgen dat katalysatoren en gasscheidingsmembranen de lichtabsorptie niet negatief beïnvloeden, en door voor goede ionengeleiding te zorgen zonder het door de zon beschenen halfgeleideroppervlak ter verkleinen of explosieve gasmengsels te produceren.

Ontwerpen met waar de onderdelen maten hebben in de ordegrootte van micrometers kunnen helpen bij het omzeilen van deze compromissen. Microfabricage is een vakgebied dat gespecialiseerd is in de parallelle fabricage van talloze micro- en nanostructuren op siliciumwafers, en silicium is een beproefd basismateriaal voor zonnepanelen en wordt ook overwogen voor gebruik in panelen die direct water splitten met behulp van zonne-energie. Microfabricagetechnieken geven echter maar een beperkte controle over de precieze vorm van siliciumstructuren in de dimensie loodrecht op het vlak van 
de wafer. Daarom zijn enkele nieuwe technieken ontwikkeld voor het patroneren van zijwanden van siliciumstructuren. Een speciale techniek die gebruik maakt van een ionenbundel onder een hoek is ontwikkeld voor het selectief wegetsen van horizontale oppervlakken, en ook is er een speciaal Bosch-etsrecept ontwikkeld voor het maken van meerdere halfronde inkepingen in verticale siliciumstructuren. In combinatie met lithografie die gebruik maakt van terugtrekking vanaf een hoek ('Retraction Edge Lithography') en lithografie die lijnen in hoeken kan definiëren ('Corner Lithography') kon selectief de top of één of meerdere banden langs de zijwand van zulke strcturen geopend worden. De technologie is robuust en precies en kan in één keer over het hele waferoppervlak velden met precies dezelfde 3D-structuren van monokristallijn silicium produceren.

De techniek voor het selectief openen van de bovenkant van een siliciumstructuur is toegepast op een fotokathode van silicium microdraden met radiale $\mathrm{n}^{+}$/p-overgangen, om zo selectief lokaal een nikkelmolybdeenlegering aan te kunnen brengen. Dit is een katalysator die alleen uit elementen bestaat die op aarde veel voorkomen, maar die geen licht doorlaat. Dankzij de selectieve depositie konden de hoeveelheid katalysator enerzijds en de hoeveelheid siliciumoppervlak waar het zonlicht op valt anderzijds los van elkaar geoptimaliseerd worden, waardoor binnen de parameterruimte de gebieden vermeden konden worden waar de efficiëntie beperkt wordt door de hoeveelheid katalysator of de hoeveelheid geabsorbeerd licht. Zo kon een efficiëntie van $10.8 \%$ behaald worden, een record voor fotokathodes gemaakt van siliciumdraden.

Er zijn stappen gezet richting een volledig geïntegreerd solar-tohydrogen device. Gebaseerd op de 'band gaps', beschikbare katalysatoren en de stabiliteit van materialen is onderzocht of een tandemconfiguratie van de zonlicht absorberende halfgeleiders silicium en hematiet volledig geïntegreerd kon worden en of ze zo samen de volledige stroom en spanning konden leveren die nodig is om water te splitsen in zuurstof en waterstof. Een verbindingslaag van $\mathrm{Ti}_{99} \mathrm{Nb}_{1}$ is ontwikkeld, en een werkend tandemdevice is gemaakt door een monokristallijne siliciumcel die niet met de omgeving in contact is te combineren met een reactief gesputterde fotoanode van hematiet. Het tandemdevice presteerde echter slecht: pas boven de $0.5 \mathrm{~V}$ vs RHE begon er een stroom te lopen en een stroom van slechts $40 \mu \mathrm{Acm}^{-2}$ werd behaald bij $1.23 \mathrm{~V}$ vs RHE. Er zijn zeker veelbelovende mogelijkhe- 
den voor het verbeteren van de prestaties, maar het blijft onzeker of zulke tandemdevices als drijvende kracht voor de elektrolyse van water ooit kunnen concurreren met trippelcellen met silicium als hoofdbestanddeel.

Een andere stap richting een volledig geïntegreerd was het onderzoek van een innovatief ontwerp gebaseerd op een membraan van silicium met daarin microporiën. Het membraan heeft geïntegreerde elektroden aan beide zijden, en kan water splitsen waarbij het voltageverlies door ionentransport onder de $100 \mathrm{mV}$ blijft bij een stroomdichtheid van $10 \mathrm{~mA} \mathrm{~cm}^{-2}$ in een elektrolyt van $1 \mathrm{M} \mathrm{NaOH}$. Dit kan bereikt worden terwijl ongeveer $99 \%$ van het oppervlak van het membraan beschikbaar blijft voor lichtabsorptie. Hoewel er geen ionenselectief membraan gebruikt werd lekte er toch maar ongeveer $1 \%$ van de geproduceerde waterstof naar het compartiment waarin de zuurstof gevormd werd. Dit laat zien hoe veelbelovend dit ontwerp is, omdat het de compromissen tussen ionentranpsport, lichtabsorptie en gasscheiding omzeilt.

Verschillende ontwerpen voor solar-to-hydrogen devices zijn overwogen, en er is beargumenteerd dat alle ontwerpen die fotoelektroden gebruiken die gemaakt zijn van op de aarde veel voorkomende elementen wel een vorm van microstructurering nodig hebben om bepaalde compromissen te omzeilen. Deze materialen kunnen vaak goedkoop gemaakt worden door ze vanuit oplossing aan te brengen, en als hun prestaties verbeterd kunnen worden zouden ze een grote bijdrage kunnen leveren aan het betaalbaar maken van waterstof die gemaakt is met behulp van zonne-energie. Moderne trippelcellen gebaseerd op silicium kunnen momenteel echter efficiënter de watersplitsreactie aandrijven, en vereisen minder integratie met het elektrolyse-gedeelte van het solar-to-hydrogen device omdat ze geen gebruik maken van een directe overgang tussen een halfgeleider en het elektrolyt. Daardoor kunnen compromissen makkelijker vermeden worden (zonder microstructurering) en de productie van zulke panelen zou makkelijker kunnen zijn. Vanuit economisch perspectief is het waarschijnlijk zelfs nog gunstiger om de zonnecellen en het elektrolysegedeelte volledig te scheiden, en ze alleen met elektrische bedrading (mogelijk via vermogenselektronica) te verbinden. Hierdoor zouden beide componenten los van elkaar geoptimaliseerd kunnen worden. Als de installatie daarnaast verbonden is met het elektriciteitsnet zou de elektriciteit ook direct gebruikt kunnen worden als de vraag 
hoog genoeg is, waardoor de verliezen waarmee de reacties voor het splitsen en recombineren van water gepaard gaan vermeden kunnen worden.

Voor de nabije toekomst lijkt de implementatie van zulke 'PV-Elektrolyse' in ieder geval waarschijnlijker dan het gebruik van verder geïntegreerde fotoelektrochemische panelen. Door de lage prijs van elektriciteit en waterstof die geproduceerd zijn met fossiele brandstoffen zullen de eerste rendabele toepassingen waarschijnlijk bestaan uit kleine installaties op plaatsen waar geen verbinding met het elektriciteitsnet mogelijk of wenselijk is, of misschien een toepassing waar duurzame waterstof gemaakt wordt als grondstof voor een chemisch proces. Echter, ontwikkelingen in fotoelektrodematerialen, katalysatoren en het ontwerp van de panelen (en de bijbehorende microstructurering), alsmede het op raken van fossiele brandstoffen en de implementatie van heffingen en subsidies die het gebruik van duurzame energie bevorderen, zouden er voor kunnen zorgen dat waterstof gemaakt met zonne-energie op de lange termijn interessant wordt voor de wereldwijde energiemarkt. 


\section{Acknowledgments}

First of all I want to thank God, the one who made and maintains everything that exists, and has given humanity a special responsibility to take good care of His creation. He has been a source of inspiration, energy, hope and comfort for me throughout my PhD project, and everything I could give and contribute in these four years I owe to Him.

In the second place I want to thank my wife Andrea for her love and support throughout these years. She has brought a lot of joy, happiness and comfort to my life in this time. I am also very happy that our first son Micha was born to us in this time, it has not made our life easier, but he brought a lot of joy. I also want to thank my parents for their continued support for us, even though this has also been a time of great sorrow for our family. And my parents in law were always interested in my progress and always supported us and visited us many times despite the distance. Here I also want to mention the small group of people from our church that we met regularly, who have practically been a family for us and have supported us a lot.

All right, now to the persons that were more directly related to my work. Of course I have to thank my supervisors Han and Jurriaan, for getting financing for this project and allowing me to waste it on many crazy ideas :-). No seriously, am very thankful for all the fruitful discussions we had over the years, together with Wouter, I really enjoyed these moments. It was good to have their knowledge and experience as a basis for my research. Also during the writing phase all their detailed corrections and suggestions were invaluable.

Yes, I already mentioned Wouter, he was truly my 'brother in arms' (as Jurriaan called it). Oh how I enjoyed all those times that we sat together and made the wildest (but not necessarily unrealistic) plans for 
experiments and devices! He was always interested in me personally and always ready for practical help. Sometimes I was a bit frustrated that again he got something to work while I was still struggling with my experiments. But after some time I began to understand that this was not caused by my poor experimental skills, but because he took a more brute force approach and was prepared to spend much more time on it. He had a similar percentage of failed experiments, he just managed to do a lot more, and that was an important asset in our joint projects. I can hardly imagine a better fellow PhD student in this project.

I want to thank Erwin for all the discussions about microfabrication. We both love it, all the process flows and ingenious tricks to make sophisticated structures with the tools that microfabrication and our cleanroom provide us, for the sheer fun of it. Yes, I will miss that. Maybe one day I can come back and we can play together again :-). But he was also invaluable for all his clean room experience, infectious laugh and friendship. And when you mention Erwin, you also mention Niels, involved in many of the microfabrication discussions with his extensive understanding of a broad range of physical and chemical phenomena. The supposedly weekly meeting supposedly starting at 9:15, where everyone present was supposed to get a turn to present their progress and supposedly ending at 10:30... I suppose this was often the highlight of my week.

I also have to thank all the students that I supervised during this project: Antoine, Ilse, Maarten, Raoul, Rianne, Salmeen, Yanfei, Julie, Ben, Daphne and Shu (I hope I am not forgetting one!). Some of them for a project of a few days, up to Shu who took a full year to complete his master thesis with me. I learned a lot from them, because for example I could apparently not explain the theory at an appropriate level or I could not get them to work at all. But I also learned from them simply because they had time to do experiments that I did not have, because they had skills that I did not have, or because they had insights that I did not have. Especially Shu I want to thank for providing me with a complete chapter for my thesis (Chapter 4).

All the experiments would not have been possible without certain people: Stefan, Pino and Henk-Willem managing the MCS/MSS labs and their practical help there, Robert Meijer, Alexander, Kai and Guido for their help in the PCS lab, and all the cleanroom staff. From the latter I want to mention especially Meint for his input to the process flows, 
Hans because many times I went to the limits of what was possible/allowed/machine-friendly with the machines that were his responsibility, and Henk for making the FIB cross sections in Chapter 3.

I am not going to try to sum up all the members of our group, Mesoscale Chemical Systems, that I had the pleasure to work with over the years, I am too afraid that I will forget someone. But it has definitely been a pleasure, and especially during all those lunch breaks with coffee, where also people from MSS were usually joined. Too bad most of the scientific staff missed this, and therefore never really got to understand the most important part of our research.

For the scientific input the TBSC group with people from different research group was closer to me than the MCS group, and I want to thank all members for their presentations and contributions. Especially Rick, Sun-Young, Janneke and Alexander I want to thank for pioneering this research topic at the University of Twente and sharing all their experience with us. 
Acknowledgments 


\section{Publications}

- Chapter 3: based on P. J. Westerik, W. J. C. Vijselaar, J. W. Berenschot, N. R. Tas, J. Huskens, and J. G. E. Gardeniers. Sidewall patterning-a new wafer-scale method for accurate patterning of vertical silicon structures. Journal of Micromechanics and Microengineering, 28(1):015008, 2017. URL https://doi.org/10.1088/ $1361-6439 / \mathrm{aa} 9 \mathrm{c} 20$

- Chapter 4: paper in preparation

- Chapter 5: based on W. Vijselaar, P. Westerik, J. Veerbeek, R. Tiggelaar, E. Berenschot, N. Tas, H. Gardeniers, and J. Huskens. Spatial decoupling of light absorption and catalytic activity of nickelmolybdenum on high-aspect-ratio silicon microwire arrays. Nature Energy, 2018. URL http://dx . doi .org/10.1038/s41560-017-0068-x

- Chapter 7: paper in preparation 
Stellingen

behorende bij het proefschrift

\title{
Microfabrication for solar-to-hydrogen devices
}

\author{
door \\ Pieter Westerik
}

1. De goede lichtabsorptie van velden met silicium micropilaren is voor een significant deel te danken aan de aanwezigheid van het substraat onder de pilaren.

2. Met de huidige prestaties van halfgeleiders gemaakt van elementen die op aarde ruim aanwezig zijn, is een begraven cel van kristallijn silicium met een p-n junctie een onmisbare component van ieder efficiënt apparaat dat zonlicht gebruikt om waterstof te maken.

3. Poriën met een diameter van enkele micrometers en een hydrofiele binnenwand die gevuld zijn met elektrolyt laten geen gasbubbels door, maar opgelost gas kan er wel doorheen diffunderen.

4. Patroneren van katalysatoren, ionentransportkanalen en licht absorberende halfgeleiders op nano- of micrometerschaal is nodig om volledig geïntegreerde watersplitstoestellen die op zonlicht werken en een halfgeleider bevatten die een junctie maakt door contact met het elektrolyt hun theoretische maximale efficiëntie te laten benaderen.

5. Door de manier waarop wetenschappelijk onderzoek in Nederland georganiseerd is gaan voor de jonge generatie wetenschappers baanperspectief op de lange termijn en een stabiel gezinsleven niet goed samen.

6. Het ideaal van reproduceerbaarheid heeft in de wetenschap het onderspit gedolven tegen een hoge druk om spannende, baanbrekende resultaten te publiceren. 


\author{
Propositions \\ belonging to the thesis
}

\title{
Microfabrication for solar-to-hydrogen devices
}

\author{
by \\ Pieter Westerik
}

1. A significant portion of the favorable light absorption properties of silicon micropillar arrays is due to the presence of the substrate under the pillars.

2. With the current performance of semiconductors made out of earth-abundant elements, a buried p-n junction crystalline silicon cell is an inevitable component of any efficient integrated solar-to-hydrogen device.

3. Electrolyte-filled pores with a diameter of several micrometers and a hydrophilic inner wall effectively prevent the passage of gas bubbles, but not diffusion dissolved gas.

4. Patterning catalysts, ion transport channels and light absorbing semiconductors at the nano or micrometer range is needed to allow fully integrated solar water splitting devices with a semiconductor-liquid junction to approach their maximum theoretical efficiency.

5. Because of how scientific research is organized in the Netherlands, for the younger generation of scientists long term job perspective and a stable family life are not easily combined.

6. The reproducibility ideal in science has been made subordinate to the pressure to publish exciting, pioneering results. 
Climate change and the depletion of tossil fuels urge us to switch to more renewable energy sources. There is more than enough sunlight to provide all the energy that we need. However, because the sun does not always shine, we need a way to store this energy for later use, solar-tohydrogen devices generate a fuel which can be stored, instead of electricity which has to be used directly when it is generated. This PhD thesis investigates the usefulness of microstructuring for such devices, microstructuring that is achieved with techniques that are also used for computer chip fabrication.

Pieter Westerik was born in 1988 in South Africa. He received his bachelor degree in Advanced Technology (2011) and his máster degree in Electrical Engineering (2014) at the University of Twente. After that he started his PhD research at the seme university in the Mesoscale Chemical Systems group of Han Gordeniers. 